\title{
Smoking and COVID Living Review (v11): a bayesian analysis
}

\author{
David Simons ${ }^{1}$, Lion Shahab ${ }^{2}$, Jamie Brown ${ }^{2}$, Olga Perski ${ }^{2}$ \\ 1 Royal Veterinary College, RVC \\ 2 University College London, University of London
}

Funding: The author(s) received no specific funding for this work.

Potential competing interests: The author(s) declared that no potential competing interests exist.

\section{Abstract}

Aims: To estimate the association of smoking status with rates of i) infection, ii) hospitalisation, iii) disease severity, and iv) mortality from SARS-CoV-2/COVID-19 disease.

Design: Living rapid review of observational and experimental studies with random-effects hierarchical Bayesian metaanalyses. Published articles and pre-prints were identified via MEDLINE and medRxiv.

Setting: Community or hospital. No restrictions on location.

Participants: Adults who received a SARS-CoV-2 test or a COVID-19 diagnosis.

Measurements: Outcomes were SARS-CoV-2 infection, hospitalisation, disease severity and mortality stratified by smoking status. Study quality was assessed (i.e. 'good', 'fair' and 'poor').

Findings: v11 (searches up to 2021-02-16) included 405 studies with 62 'good' and 'fair' quality studies included in unadjusted meta-analyses. 121 studies (29.9\%) reported current, former and never smoking status with the remainder using broader categories. Recorded smoking prevalence among people with COVID-19 was generally lower than national prevalence. Current compared with never smokers were at reduced risk of SARS-CoV-2 infection $(R R=0.71$, $95 \%$ Credible Interval $(\mathrm{Crl})=0.61-0.82, \tau=0.34)$. Data for former smokers were inconclusive $(\mathrm{RR}=1.03,95 \% \mathrm{Crl}=$ $0.95-1.11, \tau=0.17$ ) but favoured there being no important association (4\% probability of $R R \geq 1.1)$. Former compared with never smokers were at increased risk of hospitalisation $(R R=1.19, \mathrm{Crl}=1.1-1.29, \tau=0.13)$, greater disease severity $(R R=1.8, C r l=1.27-2.55, \tau=0.46)$ and mortality $(R R=1.56, C r l=1.23-2, \tau=0.43)$. Data for current smokers on hospitalisation, disease severity and mortality were inconclusive $(R R=1.1,95 \% \mathrm{Crl}=0.99-1.21, \tau=0.15 ; \mathrm{R} 1.26$, $95 \% \mathrm{Crl}=0.92-1.73, \tau=0.32 ; \mathrm{RR}=1.12,95 \% \mathrm{Crl}=0.84-1.47, \tau=0.42$, respectively) but favoured there being no important associations with hospitalisation and mortality (49\% and 56\% probability of RR $\geq 1.1$, respectively) and a small but important association with disease severity ( $83 \%$ probability of $R R \geq 1.1$ ). 
Conclusions: Compared with never smokers, current smokers appear to be at reduced risk of SARS-CoV-2 infection while former smokers appear to be at increased risk of hospitalisation, greater disease severity and mortality from COVID-19. However, it is uncertain whether these associations are causal.

v7 of this living review article has been published in $\underline{\text { Addiction }}$

\section{Introduction}

COVID-19 is a respiratory disease caused by the SARS-CoV-2 virus. Large age and gender differences in case severity and mortality have been observed in the ongoing COVID-19 pandemic (Guan, Ni, et al., 2020); however, these differences are currently unexplained. SARS-CoV-2 enters epithelial cells through the angiotensin-converting enzyme 2 (ACE-2) receptor (Hoffmann et al., 2020). Some evidence suggests that gene expression and subsequent receptor levels are elevated in the airway and oral epithelium of current smokers (Brake et al., 2020; Cai, 2020), which could put smokers at higher risk of contracting SARS-CoV-2. Other studies, however, suggest that nicotine downregulates the ACE-2 receptor (Oakes et al., 2018). These uncertainties notwithstanding, both former and current smoking is known to increase the risk of respiratory viral (Abadom et al., 2016; Denholm et al., 2010) and bacterial (Almirall et al., 1999; Feldman and Anderson, 2013) infections and is associated with worse outcomes once infected. Cigarette smoke reduces the respiratory immune defence through peri-bronchiolar inflammation and fibrosis, impaired mucociliary clearance and disruption of the respiratory epithelium (Dye and Adler, 1994). There is also reason to believe that behavioural factors (e.g. regular hand-tomouth movements) involved in smoking may increase SARS-CoV-2 infection and transmission in current smokers. However, early data from the COVID-19 pandemic have not provided clear evidence for a negative impact of current or former smoking on SARS-CoV-2 infection or COVID-19 disease outcomes, such as hospitalisation or mortality (Vardavas and Nikitara, 2020). It has also been hypothesised that nicotine might protect against a hyper-inflammatory response to SARS-CoV-2 infection, which may lead to adverse outcomes in patients with COVID-19 disease (Farsalinos, Niaura, et al., 2020).

There are several reviews that fall within the scope of smoking and COVID-19 (Alqahtani et al., 2020; Berlin et al., 2020; Emami et al., 2020; Farsalinos, Barbouni, et al., 2020; Grundy et al., 2020; Patanavanich and Glantz, 2020; Vardavas and Nikitara, 2020). We aimed to produce a rapid synthesis of available evidence pertaining to the rates of infection, hospitalisation, disease severity and mortality from SARS-CoV-2/COVID-19 stratified by smoking status. Given the increasing availability of data on this topic, this is a living review with regular updates. As evidence accumulates, the review will be expanded to include studies reporting COVID-19 outcomes by alternative nicotine use (e.g., nicotine replacement therapy or e-cigarettes).

\section{Methods}




\section{Study design}

This is a living evidence review which is updated as new evidence becomes available (Elliott et al., 2014). We adopted recommended best practice for rapid evidence reviews, which involved limiting the search to main databases and having one reviewer extract the data and another verify (Tricco et al., 2015). This study was not pre-registered but evolved from a report written for a UK medical society (Simons, Brown, et al., 2020). The most recent version of this living review is available here. Version 7 of this living review has been published in a peer-reviewed journal (Simons, Shahab, et al., 2020). A completed Preferred Reporting Items for Systematic Reviews and Meta-Analyses (PRISMA) checklist is included in Supplementary file 1.

\section{Eligibility criteria}

Studies were included if they:

1. Were primary research studies using experimental (e.g. randomised controlled trial), quasi-experimental (e.g. pre- and post-test) or observational (e.g. case-control, retrospective cohort, prospective cohort) study designs;

2. Included adults aged $16+$ years;

3. Recorded as outcome i) results of a SARS-CoV-2 diagnostic test (including antibody assays), ii) clinical diagnosis of COVID-19, iii) hospitalisation with COVID-19, iv) severity of COVID-19 disease in those hospitalised or v) mortality from COVID-19; Reported any of the outcomes of interest by self-reported or biochemically verified smoking status (e.g. current smoker, former smoker, never smoker) or current vaping and nicotine replacement therapy (NRT) use;

4. Were available in English;

5. Were published in a peer-reviewed journal, as a pre-print or a public health report by reputable bodies (e.g. governments, scientific societies).

\section{Search strategy}

The following terms were searched for in Ovid MEDLINE (2019-search date) as free text or Medical Subject Headings:

1. Tobacco Smoking/ or Smoking Cessation/ or Water Pipe Smoking/or Smoking/ or Smoking Pipes/ or Cigar Smoking/ or Smoking Prevention/ or Cigarette Smoking/ or smoking.mp. or Pipe Smoking/ or Smoking, Non-Tobacco Products/ or Smoking Water Pipes/

2. Nicotine/ or nicotine.mp. or Electronic Nicotine Delivery Systems/ or Nicotine Chewing Gum/

3. vaping.mp. or Vaping/

4. 1 or 2 or 3

5. Coronavirus/ or Severe Acute Respiratory Syndrome/ or Coronavirus Infections/ or covid.mp.

6. 4 and 5 
The following terms were searched for in titles, abstracts and full texts in medRxiv (no time limitations):

1. covid (this term captures both covid and SARS-CoV-2) AND smoking

2. covid AND nicotine

3. covid AND vaping

Additional articles/reports of interest were identified through mailing lists, Twitter, the International Severe Acute Respiratory and Emerging Infection Consortium (ISARIC) and the US Centers for Disease Control and Prevention (CDC). Where updated versions of pre-prints or public health reports were available, old versions were superseded.

\section{Selection of studies}

One reviewer screened titles, abstracts and full texts against the inclusion criteria.

\section{Data extraction}

Data were extracted by one reviewer and verified (i.e. independently checked against pre-prints and published reports) by another on i) author (year); ii) date published; iii) country; iv) study design; v) study setting; vi) sample size; vii) sex; viii) age; ix) smoking status (e.g. current, former, never, not stated, missing) and whether it was biochemically verified; $x$ ) use of alternative nicotine products; xi) SARS-CoV-2 testing; xii) SARS-CoV-2 infection; xiii) diagnosis of COVID-19; xiv) hospitalisation with COVID-19; xv) disease severity in those hospitalised with COVID-19; xvi) mortality; xvii) adjustment of smoking specific risk estimates for relevant covariates (e.g. age, sex); and xviii) whether a representative or random sampling method was used.

\section{Quality appraisal}

The quality of included studies was assessed to determine suitability for inclusion in meta-analyses. Studies were judged as 'good' quality if they: i) had $<20 \%$ missing data on smoking status and used a reliable self-report measure that distinguished between current, former and never smoking status; AND ii) used biochemical verification of smoking status and reported results from adjusted analyses; OR reported data from a representative/random sample. Studies were rated as 'fair' if they fulfilled only criterion i) and were otherwise rated as 'poor'. The quality appraisal was conducted by one reviewer and verified by a second.

\section{Evidence synthesis}

A narrative synthesis was conducted. Data from 'good' and 'fair' quality studies were pooled in R v.3.6.3 (R Team, 2013). In a living review where new data are regularly added to the analyses, it may be more appropriate to use a Bayesian (as opposed to frequentist) approach where prior knowledge is used in combination with new data to estimate a posterior risk 
distribution. A Bayesian approach mitigates the issue of performing multiple statistical tests, which can inflate family-wise error. A series of random-effects hierarchical Bayesian meta-analyses were performed with the brms (Bürkner, 2018) package to estimate the relative risk for each comparison with accompanying 95\% credible intervals (Crls). We first defined prior distributions for the true pooled effect size $(\mu)$ and the between-study heterogeneity $(\tau)$, with $\mu$ specified as a normal distribution with a mean equal to the derived point estimate from each comparison of interest in the immediately preceding version of this living review, and $\tau$ specified as a half-Cauchy distribution with a mean of 0 and standard deviation of 1. The half-Cauchy distribution was selected to reflect prior knowledge that high levels of between-study heterogeneity are more likely than lower levels. Markov Chain Monte Carlo methods (20,000 burn-ins followed by 80,000 iterations) were then used to generate a risk distribution for each study, in addition to a pooled effect for the posterior risk distribution. We report forest plots with the pooled effect for the posterior risk distribution displayed as the median relative risk with an accompanying 95\% Crls. We used the empirical cumulative distribution function (ECDF) to estimate the probability of there being a $10 \%$ reduction or $10 \%$ increase in relative risk ( $R R$ ) (i.e. $R R \geq 1.1$ or $R R \leq 0.9$ ). Due to a lack of indication as to what constitutes a clinically or epidemiologically meaningful effect (e.g. with regards to onward disease transmission or requirements for intensive care beds), we deemed a 10\% change in risk as small but important. Where data were inconclusive (as indicated by Crls crossing $R R=1.0$ ), to disambiguate whether data favoured no effect or there being a small but important association, we estimated whether there was $\geq 75 \%$ probability of $R R \geq 1.1$ or $R R \leq 0.9$.

Two sensitivity analyses were performed. First, a minimally informative prior for $\mu$ was specified as a normal distribution with a mean of 0 and standard deviation of 1 and $\tau$ as described above. Second, an informative prior as described above for $\mu$ was used with $\tau$ specified as a half-Cauchy distribution with a mean of 0.3 and standard deviation of 1 to reflect greater between-study heterogeneity.

To aid in the visualisation of smoking prevalence in the included studies, the weighted mean prevalence of current and former smoking was calculated for countries with $\geq 3$ studies and plotted for comparison with national prevalence estimates. It should be noted that prevalence estimates in the included studies were not adjusted for age, sex, socioeconomic position, or geographic region within countries.

\section{Results}

In the current review version (v11) with searches up to 2021-02-16, a total of 1133 records were identified, with 405 studies included in a narrative synthesis and 62 studies included in meta-analyses (see Figure 1). 


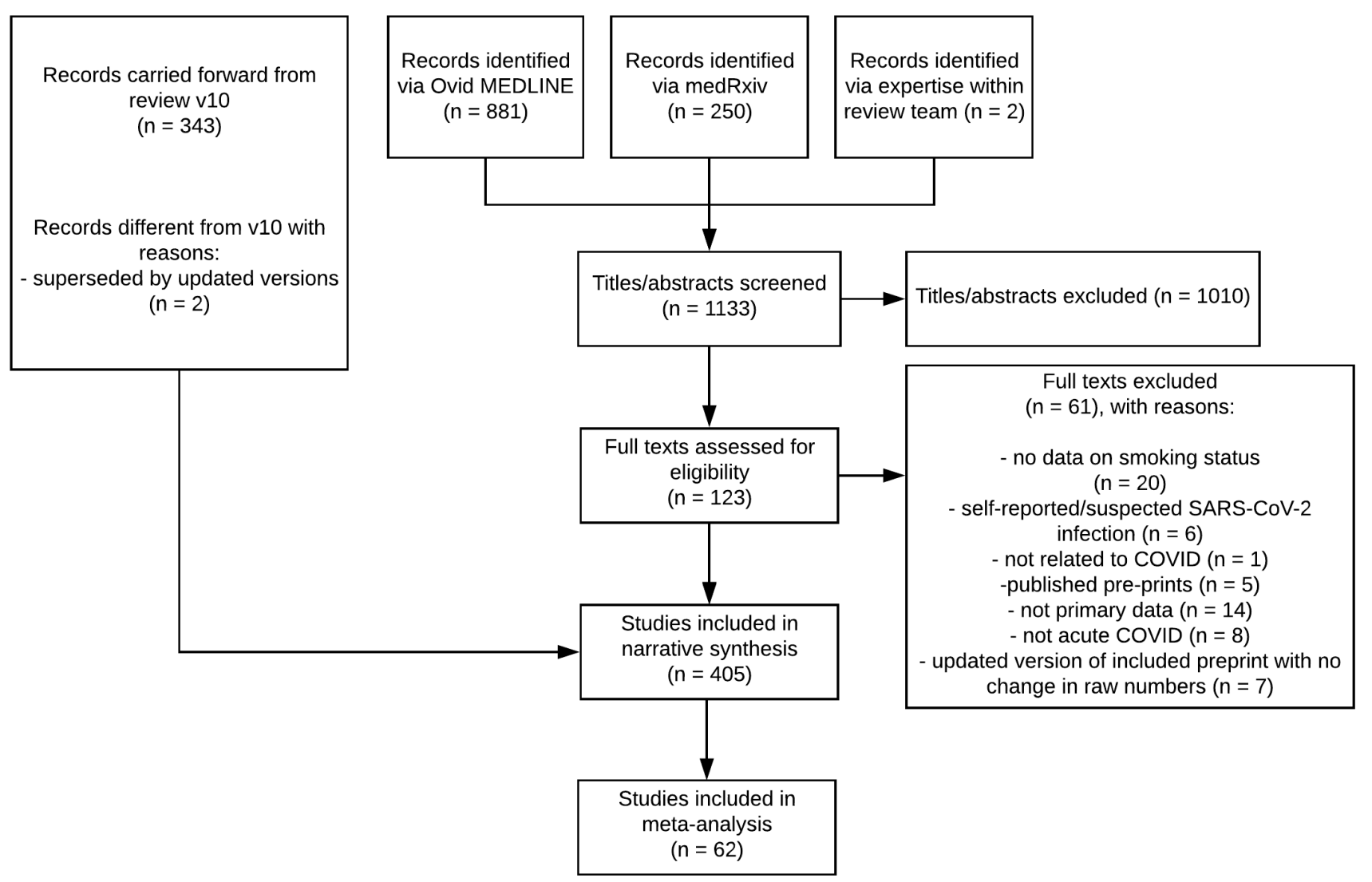

Figure 1. PRISMA flow diagram of included studies.

Characteristics of included studies are presented in Table 1. Studies were conducted across 41 countries. 109 studies were conducted in the USA, 70 in China, 44 in the UK, 28 in Spain, 20 in France, 18 in Mexico, 16 in Italy, 13 in Multiple, 8 in Turkey, 7 in Brazil and Iran, 5 in Israel and Switzerland, 4 in Finland and India, with 3 each from Australia, Austria, Japan, Saudi Arabia, and South Korea and a single study from 10 further countries. The majority of studies used observational designs (see Supplementary table S1). 256 (63.2\%) were conducted in hospital settings, 106 studies (26.2\%) included individuals from community and hospital settings, 40 studies $(9.9 \%)$ were conducted exclusively in the community, with one study each conducted in a homeless shelter and a quarantine centre, and one study that did not state the study setting. Studies had a median of 502 (interquartile range $=146-2038$ ) participants. The majority of studies (87.8\%) used reverse transcriptase polymerase chain reaction (RT-PCR) for confirmation of SARS-CoV-2 infection, $12.2 \%$ used an antibody test to confirm prior infection and $6.8 \%$ of studies relied on a combination of RT-PCR or antibody assays.

\section{Smoking status}

Categorisation of smoking status was heterogeneous (see Table 1). 236 studies collected data on smoking status through routine electronic health records (EHRs), 129 studies used a bespoke case report form for COVID-19 and 40 studies did not state the source for information on smoking status. None of the studies verified smoking status biochemically. Notably, 
only 121 (29.9\%) studies reported current, former and never smoking status (see Supplementary table S2a), with a further 26 studies reporting only ever and never smoking status (see Supplementary table S2b). The remaining 252 studies reported current, current/former or current and former smoking status but did not explicitly state whether remaining participants were never smokers or if data were missing on smoking status (see Supplementary table S2c). 133 studies explicitly reported the proportion with missing data on smoking status, which ranged from $0 \%$ to $97.6 \%$.

Use of alternative nicotine products

Eight studies recorded the use of alternative nicotine products in current and/or former smokers but did not report COVID19 outcomes stratified by alternative nicotine use (Crooks et al., 2020; Ebinger et al., 2020; Gallichotte et al., 2020; Girardeau et al., 2020; Islam et al., 2020; Kantele et al., 2020; Miyara et al., 2020; Rimland et al., 2020). One additional study grouped together current smokers and vapers (Schubl et al., 2020).

\section{Quality appraisal}

Three studies were performed in random or representative population samples and were rated as 'good' quality, and 94 studies were rated as 'fair' quality, of which 62 studies reported results stratified by smoking status for the outcomes of interest and could be included in meta-analyses. The remaining 308 studies were rated as 'poor' quality (see Table 1).

\section{Table 1. All studies included in narrative review and meta-analysis}

\begin{tabular}{|c|c|c|c|c|c|c|c|c|c|c|c|}
\hline ref & Lead author & $\begin{array}{l}\text { Date } \\
\text { published }\end{array}$ & Country & $\begin{array}{l}\text { Sample } \\
\text { size }\end{array}$ & $\begin{array}{l}\text { Study } \\
\text { setting }\end{array}$ & $\begin{array}{l}\text { Median } \\
\text { (IQR) }\end{array}$ & $\begin{array}{l}\text { Female } \\
\%\end{array}$ & $\begin{array}{l}\text { Current } \\
\text { smoker } \\
\%\end{array}$ & $\begin{array}{l}\text { Former } \\
\text { smokers } \\
\%\end{array}$ & $\begin{array}{l}\text { Current/former } \\
\text { smokers \% }\end{array}$ & $\begin{array}{l}\mathrm{Ne} \\
\mathrm{sm} \\
\%\end{array}$ \\
\hline Huang et al. (2020) & Huang, Wang & $\begin{array}{l}2020-01- \\
24\end{array}$ & China & 41 & Hospital & $\begin{array}{l}49(41- \\
58)\end{array}$ & 27.0 & 7.3 & - & - & - \\
\hline Li et al. (2020) & $\mathrm{Li}$ & $\begin{array}{l}2020-02- \\
12\end{array}$ & China & 17 & Hospital & $\begin{array}{l}45(33- \\
57)\end{array}$ & 47.1 & 17.6 & - & - & - \\
\hline Zhang et al. (2020) & Zhang, Dong & $\begin{array}{l}2020-02- \\
19\end{array}$ & China & 140 & Hospital & $\begin{array}{l}57^{\wedge} \\
(25-87)\end{array}$ & 46.3 & 1.4 & 5.00 & - & - \\
\hline Yang et al. (2020) & Yang, Yu & $\begin{array}{l}2020-02- \\
24\end{array}$ & China & 52 & Hospital & $\begin{array}{l}60(47- \\
73)\end{array}$ & 37.0 & 3.8 & - & - & - \\
\hline Guan et al. (2020) & Guan, Ni & $\begin{array}{l}2020-02- \\
28\end{array}$ & China & 1,099 & Hospital & $\begin{array}{l}47(35- \\
58)\end{array}$ & 41.9 & 12.5 & 1.91 & - & 84. \\
\hline Liu et al. (2020) & Liu, Tao & $\begin{array}{l}2020-02- \\
28\end{array}$ & China & 78 & Hospital & $\begin{array}{l}38 \text { (33- } \\
57)\end{array}$ & 50.0 & - & - & 6.41 & - \\
\hline Qi et al. (2020) & Qi & $\begin{array}{l}2020-03- \\
03\end{array}$ & China & 267 & Hospital & $\begin{array}{l}48 \text { (35- } \\
65)\end{array}$ & 45.2 & 19.9 & - & - & - \\
\hline Huang et al. (2020) & Huang, Yang & $\begin{array}{l}2020-03- \\
05\end{array}$ & China & 36 & Hospital & $\begin{array}{l}69(60- \\
78)\end{array}$ & 30.6 & - & - & 11.11 & - \\
\hline Xu et al. (2020) & $\mathrm{Xu}$ & $\begin{array}{l}2020-03- \\
08\end{array}$ & China & 53 & Hospital & NA & 47.2 & 11.3 & - & - & - \\
\hline Zhou et al. (2020) & Zhou, Yu & 2020-03- & China & 191 & Hospital & 56 (46- & 38.0 & 5.8 & - & - & - \\
\hline
\end{tabular}




\begin{tabular}{|c|c|c|c|c|c|c|c|c|c|c|c|}
\hline & & & & & & Uil & & & & & \\
\hline Liu et al. (2020) & Liu, Ming & $\begin{array}{l}2020-03- \\
12\end{array}$ & China & 41 & Hospital & $\begin{array}{l}39(30- \\
48)\end{array}$ & 58.5 & 9.8 & - & - & - \\
\hline Shi et al. (2020) & Shi, Yu & $\begin{array}{l}2020-03- \\
18\end{array}$ & China & 487 & Hospital & $\begin{array}{l}46(27- \\
65)\end{array}$ & 46.8 & - & - & 8.21 & - \\
\hline Zhang et al. (2020) & Zhang, Cai & $\begin{array}{l}2020-03- \\
20\end{array}$ & China & 645 & Hospital & NA & 49.1 & 6.4 & - & - & - \\
\hline Dong et al. (2020) & Dong, Cao & $\begin{array}{l}2020-03- \\
20\end{array}$ & China & 9 & Hospital & $\begin{array}{l}44(30- \\
46)\end{array}$ & 66.7 & 11.1 & - & - & - \\
\hline Wan et al. (2020) & Wan & $\begin{array}{l}2020-03- \\
21\end{array}$ & China & 135 & Hospital & $\begin{array}{l}47(36- \\
55)\end{array}$ & 46.7 & 6.7 & - & - & - \\
\hline Jin et al. (2020) & Jin & $\begin{array}{l}2020-03- \\
24\end{array}$ & China & 651 & Hospital & $\begin{array}{l}46(32- \\
60)\end{array}$ & 49.2 & 6.3 & - & - & - \\
\hline Wang et al. (2020) & Wang, Pan & $\begin{array}{l}2020-03- \\
24\end{array}$ & China & 125 & Hospital & $\begin{array}{l}41(26- \\
66)\end{array}$ & 43.2 & - & - & 12.80 & - \\
\hline Lian et al. (2020) & Lian & $\begin{array}{l}2020-03- \\
25\end{array}$ & China & 788 & Hospital & NA & 38.5 & 6.9 & - & - & - \\
\hline Hu et al. (2020) & $\mathrm{Hu}$ & $\begin{array}{l}2020-03- \\
25\end{array}$ & China & 323 & Hospital & $\begin{array}{l}61^{\wedge} \\
(23-91)\end{array}$ & 48.6 & - & - & 11.76 & - \\
\hline Guan et al. (2020) & Guan, Liang & $\begin{array}{l}2020-03- \\
26\end{array}$ & China & 1,590 & Hospital & $\begin{array}{l}49(33- \\
64)\end{array}$ & 42.7 & - & - & 6.98 & 93. \\
\hline Chen et al. (2020) & Chen & $\begin{array}{l}2020-03- \\
26\end{array}$ & China & 548 & Hospital & $\begin{array}{l}62(44- \\
70)\end{array}$ & 37.6 & 4.4 & 2.55 & - & - \\
\hline $\begin{array}{l}\text { CDCMMWR } \\
(2020)\end{array}$ & Chow (US CDC) & $\begin{array}{l}2020-03- \\
31\end{array}$ & USA & 7,162 & $\begin{array}{l}\text { Community } \\
\text { and } \\
\text { Hospital }\end{array}$ & NA & - & 1.3 & 2.30 & - & - \\
\hline Kim et al. (2020) & Kim & $\begin{array}{l}2020-04- \\
01\end{array}$ & $\begin{array}{l}\text { South } \\
\text { Korea }\end{array}$ & 28 & Hospital & $\begin{array}{l}43(30- \\
56)\end{array}$ & 46.4 & 17.9 & - & - & - \\
\hline Feng et al. (2020) & Feng & $\begin{array}{l}2020-04- \\
10\end{array}$ & China & 476 & Hospital & $\begin{array}{l}53(40- \\
64)\end{array}$ & 43.1 & 9.2 & - & - & - \\
\hline $\begin{array}{l}\text { Rentsch et al. } \\
(2020)\end{array}$ & Rentsch & $\begin{array}{l}2020-04- \\
14\end{array}$ & USA & 3,528 & $\begin{array}{l}\text { Community } \\
\text { and } \\
\text { Hospital }\end{array}$ & $\begin{array}{l}66(60- \\
70)\end{array}$ & 4.6 & 27.2 & 30.60 & - & 36. \\
\hline Goyal et al. (2020) & Goyal & $\begin{array}{l}2020-04- \\
17\end{array}$ & USA & 393 & Hospital & $\begin{array}{l}62.2 \\
(49-74)\end{array}$ & 39.3 & 5.1 & - & - & - \\
\hline Zheng et al. (2020) & Zheng, Gao & $\begin{array}{l}2020-04- \\
19\end{array}$ & China & 66 & Hospital & $\begin{array}{l}47^{\wedge} \\
(\mathrm{NA})\end{array}$ & 25.8 & 12.1 & - & - & - \\
\hline Gold et al. (2020) & Gold (US CDC) & $\begin{array}{l}2020-04- \\
20\end{array}$ & USA & 305 & Hospital & NA & 50.5 & 5.2 & - & - & - \\
\hline $\begin{array}{l}\text { Argenziano et al. } \\
\text { (2020) }\end{array}$ & Argenziano & $\begin{array}{l}2020-04- \\
22\end{array}$ & USA & 1,000 & Hospital & $\begin{array}{l}63(50- \\
75)\end{array}$ & 40.4 & 4.9 & 17.90 & - & 77. \\
\hline $\begin{array}{l}\text { Richardson et al. } \\
(2020)\end{array}$ & Richardson & $\begin{array}{l}2020-04- \\
22\end{array}$ & USA & 5,700 & Hospital & $\begin{array}{l}63(52- \\
75)\end{array}$ & 39.7 & - & - & 9.79 & 52. \\
\hline $\begin{array}{l}\text { Fontanet et al. } \\
(2020)\end{array}$ & Fontanet & $\begin{array}{l}2020-04- \\
23\end{array}$ & France & 661 & $\begin{array}{l}\text { Community } \\
\text { and } \\
\text { Hospital }\end{array}$ & $\begin{array}{l}37(16- \\
47)\end{array}$ & 62.0 & 10.4 & - & - & - \\
\hline
\end{tabular}




\begin{tabular}{|c|c|c|c|c|c|c|c|c|c|c|c|}
\hline $\begin{array}{l}\text { Hadjadj et al. } \\
(2020)\end{array}$ & Hadjadj & $\begin{array}{l}2020-04- \\
23\end{array}$ & France & 50 & Hospital & $\begin{array}{l}55(50- \\
63)\end{array}$ & 22.0 & 2.0 & 18.00 & - & 80. \\
\hline Liao et al. (2020) & Liao, Feng & $\begin{array}{l}2020-04- \\
24\end{array}$ & China & 1,848 & Hospital & $\begin{array}{l}55(48- \\
61)\end{array}$ & 54.7 & - & - & 0.43 & - \\
\hline $\begin{array}{l}\text { Gil-Agudo et al. } \\
(2020)\end{array}$ & Gil-Agudo & $\begin{array}{l}2020-04- \\
24\end{array}$ & Spain & 7 & Hospital & $\begin{array}{l}68(34- \\
75)\end{array}$ & 28.6 & - & - & 42.86 & 57. \\
\hline Yao et al. (2020) & Yao & $\begin{array}{l}2020-04- \\
24\end{array}$ & China & 108 & Hospital & $\begin{array}{l}52(37- \\
58)\end{array}$ & 60.2 & 3.7 & - & - & - \\
\hline Zuo et al. (2020) & Zuo, Yalavarthi & $\begin{array}{l}2020-04- \\
24\end{array}$ & USA & 50 & Hospital & $\begin{array}{l}61(46- \\
76)\end{array}$ & 34.0 & - & - & 36.00 & - \\
\hline $\begin{array}{l}\text { Solís and Carreño } \\
(2020)\end{array}$ & Solis & $\begin{array}{l}2020-04- \\
25\end{array}$ & Mexico & 650 & Hospital & $\begin{array}{l}46 \\
\text { (NA) }\end{array}$ & 42.1 & 9.4 & - & - & - \\
\hline Yu et al. (2020) & Yu, Cai & $\begin{array}{l}2020-04- \\
27\end{array}$ & China & 95 & Hospital & NA & 44.2 & 8.4 & - & - & - \\
\hline Ziehr et al. (2020) & Ziehr & $\begin{array}{l}2020-04- \\
29\end{array}$ & USA & 66 & Hospital & $\begin{array}{l}58(23- \\
87)\end{array}$ & 35.0 & - & - & 33.33 & 63. \\
\hline Zheng et al. (2020) & Zheng, Xiong & $\begin{array}{l}2020-04- \\
30\end{array}$ & China & 73 & Hospital & $\begin{array}{l}43^{\wedge} \\
(\mathrm{NA})\end{array}$ & 45.2 & - & - & 10.96 & 89. \\
\hline Kalan et al. (2020) & Kalan & $\begin{array}{l}2020-05- \\
01\end{array}$ & Iran & 193 & Hospital & $\begin{array}{l}52.6^{\wedge} \\
(37-67)\end{array}$ & 36.3 & 7.3 & - & - & 85. \\
\hline Kolin et al. (2020) & Kolin & $\begin{array}{l}2020-05- \\
05\end{array}$ & UK & 1,474 & $\begin{array}{l}\text { Community } \\
\text { and } \\
\text { Hospital }\end{array}$ & $\begin{array}{l}58(49- \\
67)\end{array}$ & 46.6 & 14.5 & 40.16 & - & 44. \\
\hline $\begin{array}{l}\text { Borobia et al. } \\
(2020)\end{array}$ & Borobia & $\begin{array}{l}2020-05- \\
06\end{array}$ & Spain & 2,226 & Hospital & $\begin{array}{l}61(46- \\
78)\end{array}$ & 52.0 & 7.1 & - & - & - \\
\hline $\begin{array}{l}\text { Giacomelli et al. } \\
(2020)\end{array}$ & Giacomelli & $\begin{array}{l}2020-05- \\
06\end{array}$ & Italy & 233 & Hospital & $\begin{array}{l}61(50- \\
72)\end{array}$ & 31.9 & - & - & 30.04 & 70. \\
\hline Shah et al. (2020) & Shah & $\begin{array}{l}2020-05- \\
06\end{array}$ & USA & 316 & Hospital & $\begin{array}{l}63(43- \\
72)\end{array}$ & 48.1 & 16.5 & 17.72 & - & 42. \\
\hline $\begin{array}{l}\text { Williamson et al. } \\
(2020)\end{array}$ & $\begin{array}{l}\text { The OpenSAFELY } \\
\text { Collaborative }\end{array}$ & $\begin{array}{l}2020-05- \\
07\end{array}$ & UK & $17,425,445$ & $\begin{array}{l}\text { Community } \\
\text { and } \\
\text { Hospital }\end{array}$ & NA & 50.1 & 17.0 & 32.93 & - & 45. \\
\hline $\begin{array}{l}\text { Allenbach et al. } \\
(2020)\end{array}$ & Allenbach & $\begin{array}{l}2020-05- \\
08\end{array}$ & France & 152 & Hospital & $\begin{array}{l}77(60- \\
83)\end{array}$ & 31.1 & - & - & 6.58 & - \\
\hline $\begin{array}{l}\text { Robilotti et al. } \\
\text { (2020) }\end{array}$ & Robilotti & $\begin{array}{l}2020-05- \\
08\end{array}$ & USA & 423 & Hospital & NA & 50.0 & 2.1 & 37.59 & - & 58. \\
\hline $\begin{array}{l}\text { Lubetzky et al. } \\
(2020)\end{array}$ & Lubetzky & $\begin{array}{l}2020-05- \\
08\end{array}$ & USA & 54 & Hospital & $\begin{array}{l}57(29- \\
83)\end{array}$ & 62.0 & - & - & 22.22 & - \\
\hline Yin et al. (2020) & Yin, Yang & $\begin{array}{l}2020-05- \\
10\end{array}$ & China & 106 & Hospital & $\begin{array}{l}73(61- \\
85)\end{array}$ & 39.6 & - & - & 16.98 & - \\
\hline Rica et al. (2020) & de la Rica & $\begin{array}{l}2020-05- \\
11\end{array}$ & Spain & 48 & Hospital & $\begin{array}{l}66^{\wedge} \\
(33-88)\end{array}$ & 33.0 & - & - & 20.83 & - \\
\hline Cho et al. (2020) & Cho & $\begin{array}{l}2020-05- \\
11\end{array}$ & UK & 1,331 & $\begin{array}{l}\text { Community } \\
\text { and } \\
\text { Hospital }\end{array}$ & NA & 49.2 & 19.0 & 26.97 & - & 54. \\
\hline $\begin{array}{l}\text { Yanover et al. } \\
(2020)\end{array}$ & Yanover & $\begin{array}{l}2020-05- \\
13\end{array}$ & Israel & 4,353 & $\begin{array}{l}\text { Community } \\
\text { and } \\
\text { Hospital }\end{array}$ & $\begin{array}{l}35(22- \\
54)\end{array}$ & 44.5 & 11.8 & 2.96 & - & 85. \\
\hline Hamer et al. (2020) & Hamer & $\begin{array}{l}2020-05- \\
13\end{array}$ & UK & 387,109 & Hospital & $\begin{array}{l}56.2 \\
(48-64)\end{array}$ & 55.1 & 9.7 & 34.84 & - & 55. \\
\hline Targher et al. & Taraher & 2020-05- & China & 339 & Hosnital & $48.4^{\wedge}$ & 52.8 & 8.3 & & - & - \\
\hline
\end{tabular}




\begin{tabular}{|c|c|c|c|c|c|c|c|c|c|c|}
\hline (2020) & $x^{2}$ & 13 & - n... & ... & (neran & (NA) & $\cdots$ & $\cdots$ & & \\
\hline $\begin{array}{l}\text { Carrillo-Vega et al. } \\
(2020)\end{array}$ & Carillo-Vega & $\begin{array}{l}2020-05- \\
14\end{array}$ & Mexico & 10,544 & $\begin{array}{l}\text { Community } \\
\text { and } \\
\text { Hospital }\end{array}$ & $\begin{array}{l}46.5^{\wedge} \\
(30-62)\end{array}$ & 42.3 & 8.9 & - & - \\
\hline $\begin{array}{l}\text { Regina et al. } \\
(2020)\end{array}$ & Regina & $\begin{array}{l}2020-05- \\
14\end{array}$ & Switzerland & 200 & Hospital & $\begin{array}{l}70(55- \\
81)\end{array}$ & 40.0 & 4.5 & - & - \\
\hline $\begin{array}{l}\text { Almazeedi et al. } \\
(2020)\end{array}$ & Almazeedi & $\begin{array}{l}2020-05- \\
15\end{array}$ & Kuwait & 1,096 & Hospital & $\begin{array}{l}41(25- \\
57)\end{array}$ & 19.0 & 4.0 & - & - \\
\hline $\begin{array}{l}\text { Lusignan et al. } \\
(2020)\end{array}$ & de Lusignan & $\begin{array}{l}2020-05- \\
15\end{array}$ & UK & 3,802 & Community & $\begin{array}{l}58(34- \\
73)\end{array}$ & 57.6 & 10.9 & 46.11 & - \\
\hline $\begin{array}{l}\text { Palaiodimos et al. } \\
(2020)\end{array}$ & Palaiodimos & $\begin{array}{l}2020-05- \\
15\end{array}$ & USA & 200 & Hospital & $\begin{array}{l}64(50- \\
73.5)\end{array}$ & 51.0 & - & - & 32.50 \\
\hline $\begin{array}{l}\text { Mejia-Vilet et al. } \\
(2020)\end{array}$ & Mejia-Vilet & $\begin{array}{l}2020-05- \\
16\end{array}$ & Mexico & 329 & Hospital & $\begin{array}{l}49(41- \\
60)\end{array}$ & 36.0 & - & - & 6.99 \\
\hline Chen et al. (2020) & Chen, Jiang & $\begin{array}{l}2020-05- \\
16\end{array}$ & China & 135 & Hospital & NA & 42.2 & - & - & 9.63 \\
\hline Li et al. (2020) & Li, Chen & $\begin{array}{l}2020-05- \\
16\end{array}$ & China & 1,008 & Hospital & $\begin{array}{l}55(44- \\
65)\end{array}$ & 43.6 & 5.7 & - & - \\
\hline $\begin{array}{l}\text { Valenti et al. } \\
(2020)\end{array}$ & Valenti & $\begin{array}{l}2020-05- \\
18\end{array}$ & Italy & 789 & Community & $\begin{array}{l}40.7^{\wedge} \\
(\mathrm{NA})\end{array}$ & 35.0 & 25.9 & - & - \\
\hline Feuth et al. (2020) & Feuth & $\begin{array}{l}2020-05- \\
18\end{array}$ & Finland & 28 & Hospital & $\begin{array}{l}56(47- \\
72)\end{array}$ & 46.0 & 10.7 & 28.57 & - \\
\hline Ge et al. (2020) & $\mathrm{Ge}$ & $\begin{array}{l}2020-05- \\
18\end{array}$ & China & 51 & Hospital & $\begin{array}{l}70(58- \\
79)\end{array}$ & 27.5 & 13.7 & - & - \\
\hline $\begin{array}{l}\text { Parrotta et al. } \\
(2020)\end{array}$ & Parrotta & $\begin{array}{l}2020-05- \\
18\end{array}$ & USA & 76 & $\begin{array}{l}\text { Community } \\
\text { and } \\
\text { Hospital }\end{array}$ & $\begin{array}{l}44.9 \\
(13-71)\end{array}$ & 61.8 & 2.6 & 26.32 & - \\
\hline $\begin{array}{l}\text { Shekhar et al. } \\
\text { (2020) }\end{array}$ & Shekhar & $\begin{array}{l}2020-05- \\
18\end{array}$ & USA & 50 & Hospital & $\begin{array}{l}55.5 \\
(20-85)\end{array}$ & 54.0 & 48.0 & - & - \\
\hline $\begin{array}{l}\text { Rimland et al. } \\
\text { (2020) }\end{array}$ & Rimland & $\begin{array}{l}2020-05- \\
19\end{array}$ & USA & 11 & Hospital & $\begin{array}{l}59(48- \\
65)\end{array}$ & 18.2 & 9.1 & - & - \\
\hline Basse et al. (2020) & Basse & $\begin{array}{l}2020-05- \\
19\end{array}$ & France & 141 & Hospital & $\begin{array}{l}62(52- \\
72)\end{array}$ & 72.0 & 17.7 & - & - \\
\hline Freites et al. (2020) & Freites & $\begin{array}{l}2020-05- \\
19\end{array}$ & Spain & 123 & Hospital & $\begin{array}{l}59.88^{\wedge} \\
(44-74)\end{array}$ & 69.9 & 3.3 & - & - \\
\hline $\begin{array}{l}\text { Alshami et al. } \\
(2020)\end{array}$ & Alshami & $\begin{array}{l}2020-05- \\
19\end{array}$ & $\begin{array}{l}\text { Saudi } \\
\text { Arabia }\end{array}$ & 128 & $\begin{array}{l}\text { Quarantine } \\
\text { Centre }\end{array}$ & $\begin{array}{l}39.6^{\wedge} \\
(24-55)\end{array}$ & 53.9 & 15.6 & 2.34 & \\
\hline Shi et al. (2020) & Shi, Zhao & $\begin{array}{l}2020-05- \\
20\end{array}$ & China & 101 & Hospital & $\begin{array}{l}71(59- \\
80)\end{array}$ & 40.6 & - & - & 4.95 \\
\hline $\begin{array}{l}\text { Al-Hindawi et al. } \\
(2020)\end{array}$ & Al-Hindawi & $\begin{array}{l}2020-05- \\
20\end{array}$ & UK & 31 & Hospital & $\begin{array}{l}61 \\
\text { (NA) }\end{array}$ & 12.9 & 3.2 & 70.97 & - \\
\hline Wu et al. (2020) & Wu & $\begin{array}{l}2020-05- \\
21\end{array}$ & Italy & 174 & Hospital & $\begin{array}{l}61.2^{\wedge} \\
(50-71)\end{array}$ & 30.5 & - & - & 33.33 \\
\hline Kim et al. (2020) & Kim, Garg & $\begin{array}{l}2020-05- \\
22\end{array}$ & USA & 2,491 & Hospital & $\begin{array}{l}62(50- \\
75)\end{array}$ & 46.8 & 6.0 & 25.77 & - \\
\hline $\begin{array}{l}\text { Docherty et al. } \\
(2020)\end{array}$ & Docherty & $\begin{array}{l}2020-05- \\
22\end{array}$ & Multiple & 20,133 & Hospital & $\begin{array}{l}72.9 \\
(58-82)\end{array}$ & 40.0 & 4.2 & 21.68 & - \\
\hline Petrilli et al. (2020) & Petrilli & $\begin{array}{l}2020-05- \\
22\end{array}$ & USA & 5,279 & $\begin{array}{l}\text { Community } \\
\text { and } \\
\text { Hospital }\end{array}$ & $\begin{array}{l}54(38- \\
66)\end{array}$ & 51.5 & 5.5 & 17.09 & - \\
\hline Klang et al. (n.d.) & Klang & 2020-05- & USA & 3,406 & Hospital & NA & 61.8 & - & - & 23.28 \\
\hline
\end{tabular}

29.

67.

60. 


\begin{tabular}{|c|c|c|c|c|c|c|c|c|c|c|}
\hline $\begin{array}{l}\text { Vaquero et al. } \\
(2020)\end{array}$ & Vaquero-Roncero & $\begin{array}{l}2020-05- \\
24\end{array}$ & Spain & 146 & Hospital & $\begin{array}{l}66^{\wedge} \\
(59-72)\end{array}$ & 32.2 & - & - & 6.85 \\
\hline Ip et al. (2020) & Ip & $\begin{array}{l}2020-05- \\
25\end{array}$ & USA & 2,512 & Hospital & $\begin{array}{l}64(52- \\
76)\end{array}$ & 37.6 & 3.1 & 17.83 & - \\
\hline $\begin{array}{l}\text { Heili-Frades } \\
(2020)\end{array}$ & Heili-Frades & $\begin{array}{l}2020-05- \\
25\end{array}$ & Spain & 4,712 & Hospital & $\begin{array}{l}62(47- \\
77)\end{array}$ & 50.5 & 4.9 & 17.40 & - \\
\hline $\begin{array}{l}\text { Berumen et al. } \\
(2020)\end{array}$ & Berumen & $\begin{array}{l}2020-05- \\
26\end{array}$ & Mexico & 102,875 & Hospital & NA & 49.1 & - & - & 9.64 \\
\hline $\begin{array}{l}\text { Garibaldi et al. } \\
(2020)\end{array}$ & Garibaldi & $\begin{array}{l}2020-05- \\
26\end{array}$ & USA & 832 & Hospital & $\begin{array}{l}63(49- \\
75)\end{array}$ & 47.0 & 5.5 & 22.60 & - \\
\hline $\begin{array}{l}\text { Soto-Mota et al. } \\
(2020)\end{array}$ & Soto-Mota & $\begin{array}{l}2020-05- \\
27\end{array}$ & Mexico & 400 & Hospital & NA & 30.0 & - & - & 12.00 \\
\hline Li et al. (2020) & Li, Long & $\begin{array}{l}2020-05- \\
28\end{array}$ & China & 145 & Not Stated & $\begin{array}{l}49^{\wedge} \\
(13-80)\end{array}$ & 61.0 & - & - & 5.52 \\
\hline Louis et al. (2020) & Louis & $\begin{array}{l}2020-05- \\
28\end{array}$ & USA & 22 & Hospital & $\begin{array}{l}66.5^{\wedge} \\
(55-77)\end{array}$ & 36.4 & - & - & 45.45 \\
\hline $\begin{array}{l}\text { Kuderer et al. } \\
(2020)\end{array}$ & Kuderer & $\begin{array}{l}2020-05- \\
28\end{array}$ & Multiple & 928 & $\begin{array}{l}\text { Community } \\
\text { and } \\
\text { Hospital }\end{array}$ & $\begin{array}{l}66(57- \\
76)\end{array}$ & 50.0 & 4.6 & 35.13 & - \\
\hline $\begin{array}{l}\text { Gianfrancesco et } \\
\text { al. (2020) }\end{array}$ & Gianfrancesco & $\begin{array}{l}2020-05- \\
29\end{array}$ & Multiple & 600 & $\begin{array}{l}\text { Community } \\
\text { and } \\
\text { Hospital }\end{array}$ & $\begin{array}{l}56(45- \\
67)\end{array}$ & 71.0 & - & - & 21.50 \\
\hline $\begin{array}{l}\text { Chaudhry et al. } \\
\text { (2020) }\end{array}$ & Chaudhry & $\begin{array}{l}2020-05- \\
29\end{array}$ & USA & 40 & $\begin{array}{l}\text { Community } \\
\text { and } \\
\text { Hospital }\end{array}$ & $\begin{array}{l}52 \\
(45.5- \\
61)\end{array}$ & 60.0 & - & - & 15.00 \\
\hline $\begin{array}{l}\text { Niedzwiedz et al. } \\
(2020)\end{array}$ & Niedzwiedz & $\begin{array}{l}2020-05- \\
29\end{array}$ & UK & 392,116 & $\begin{array}{l}\text { Community } \\
\text { and } \\
\text { Hospital }\end{array}$ & NA & 54.9 & 9.8 & 34.81 & - \\
\hline Valle et al. (2020) & del Valle & $\begin{array}{l}2020-05- \\
30\end{array}$ & USA & 1,484 & Hospital & $\begin{array}{l}62(52- \\
72)\end{array}$ & 40.6 & 5.5 & 23.32 & - \\
\hline $\begin{array}{l}\text { Bello-Chavolla et } \\
\text { al. (2020) }\end{array}$ & Bello-Chavolla & $\begin{array}{l}2020-05- \\
31\end{array}$ & Mexico & 177,133 & $\begin{array}{l}\text { Community } \\
\text { and } \\
\text { Hospital }\end{array}$ & $\begin{array}{l}42.6 \\
(26-59)\end{array}$ & 48.9 & - & - & 9.28 \\
\hline Batty et al. (2020) & Batty & $\begin{array}{l}2020-06- \\
01\end{array}$ & UK & 908 & Hospital & $\begin{array}{l}57.27^{\wedge} \\
(48-66)\end{array}$ & 44.3 & 11.2 & - & - \\
\hline Israel et al. (2020) & Israel & $\begin{array}{l}2020-06- \\
01\end{array}$ & Israel & 24,906 & $\begin{array}{l}\text { Community } \\
\text { and } \\
\text { Hospital }\end{array}$ & $\begin{array}{l}40(27- \\
59)\end{array}$ & 48.7 & 16.8 & 12.66 & - \\
\hline Hao et al. (2020) & Hao & $\begin{array}{l}2020-06- \\
01\end{array}$ & China & 788 & Hospital & $\begin{array}{l}46(35- \\
56)\end{array}$ & 48.4 & 6.9 & - & - \\
\hline $\begin{array}{l}\text { Lassale et al. } \\
(2020)\end{array}$ & Lassale & $\begin{array}{l}2020-06- \\
01\end{array}$ & UK & 900 & Hospital & $\begin{array}{l}57.2^{\wedge} \\
(48-66)\end{array}$ & 44.4 & 11.4 & 41.89 & - \\
\hline $\begin{array}{l}\text { Eugen-Olsen et al. } \\
\text { (2020) }\end{array}$ & Eugen-Olsen & $\begin{array}{l}2020-06- \\
02\end{array}$ & Denmark & 407 & Hospital & $\begin{array}{l}64(47- \\
77)\end{array}$ & 57.7 & 20.6 & 36.86 & - \\
\hline $\begin{array}{l}\text { Martinez-Portilla et } \\
\text { al. (2020) }\end{array}$ & Martinez-Portilla & $\begin{array}{l}2020-06- \\
02\end{array}$ & Mexico & 224 & $\begin{array}{l}\text { Community } \\
\text { and } \\
\text { Hospital }\end{array}$ & $\begin{array}{l}29(26- \\
33)\end{array}$ & 100.0 & - & - & 3.12 \\
\hline $\begin{array}{l}\text { Raisi-Estabragh et } \\
\text { al. (2020) }\end{array}$ & Raisi-Estabragh & $\begin{array}{l}2020-06- \\
02\end{array}$ & UK & 4,510 & Hospital & NA & 48.8 & - & - & 51.80 \\
\hline Luo et al. (2020) & Luo & $\begin{array}{l}2020-06- \\
02\end{array}$ & China & 625 & Hospital & $\begin{array}{l}46 \\
(\mathrm{NA})\end{array}$ & 47.7 & 3.0 & - & - \\
\hline Dar......nnm ntal & & מחกח ne & & & & 1ก & & & & \\
\hline
\end{tabular}




\begin{tabular}{|c|c|c|c|c|c|c|c|c|c|c|}
\hline $\begin{array}{l}\text { Duuivvale et al. } \\
(2020)\end{array}$ & Boulware & $\begin{array}{l}\angle U<U-U 0^{-} \\
03\end{array}$ & Multiple & 821 & Community & $\begin{array}{l}40 \text { (00- } \\
50)\end{array}$ & 51.6 & 3.3 & - & - \\
\hline $\begin{array}{l}\text { Ikitimur et al. } \\
(2020)\end{array}$ & Ikitimur & $\begin{array}{l}2020-06- \\
03\end{array}$ & Turkey & 81 & Hospital & $\begin{array}{l}55^{\wedge} \\
(38-72)\end{array}$ & 44.0 & - & - & 28.40 \\
\hline $\begin{array}{l}\text { Sierpiński et al. } \\
(2020)\end{array}$ & Sierpinski & $\begin{array}{l}2020-06- \\
03\end{array}$ & Poland & 1,942 & Community & $\begin{array}{l}50 \\
\text { (NA) }\end{array}$ & 60.0 & 6.3 & - & - \\
\hline Wang et al. (2020) & Wang, Oekelen & $\begin{array}{l}2020-06- \\
05\end{array}$ & USA & 58 & $\begin{array}{l}\text { Community } \\
\text { and } \\
\text { Hospital }\end{array}$ & $\begin{array}{l}67 \\
\text { (NA) }\end{array}$ & 48.0 & - & - & 36.21 \\
\hline $\begin{array}{l}\text { Perrone et al. } \\
(2020)\end{array}$ & Perrone & $\begin{array}{l}2020-06- \\
05\end{array}$ & Italy & 1,189 & Hospital & NA & 21.2 & - & - & 21.87 \\
\hline $\begin{array}{l}\text { Sharma et al. } \\
(2020)\end{array}$ & Sharma & $\begin{array}{l}2020-06- \\
05\end{array}$ & India & 501 & Hospital & $\begin{array}{l}35.1^{\wedge} \\
(18-51)\end{array}$ & 36.0 & - & - & 4.19 \\
\hline $\begin{array}{l}\text { Magagnoli et al. } \\
(2020)\end{array}$ & Magagnoli & $\begin{array}{l}2020-06- \\
05\end{array}$ & USA & 807 & Hospital & $\begin{array}{l}70(60- \\
75)\end{array}$ & 4.3 & - & - & 15.86 \\
\hline $\begin{array}{l}\text { Ramlall et al. } \\
(2020)\end{array}$ & Ramlall & $\begin{array}{l}2020-06- \\
06\end{array}$ & USA & 11,116 & $\begin{array}{l}\text { Community } \\
\text { and } \\
\text { Hospital }\end{array}$ & $\begin{array}{l}52 \\
(34.7- \\
69.5)\end{array}$ & 55.2 & - & - & 26.80 \\
\hline $\begin{array}{l}\text { Giannouchos et al. } \\
(2020)\end{array}$ & Giannouchos & $\begin{array}{l}2020-06- \\
07\end{array}$ & Mexico & 236,439 & $\begin{array}{l}\text { Community } \\
\text { and } \\
\text { Hospital }\end{array}$ & $\begin{array}{l}42.5^{\wedge} \\
(25-59)\end{array}$ & 49.1 & 9.1 & - & - \\
\hline $\begin{array}{l}\text { Romão et al. } \\
\text { (2020) }\end{array}$ & Romao & $\begin{array}{l}2020-06- \\
08\end{array}$ & Portugal & 34 & Community & $\begin{array}{l}41^{\wedge} \\
(26-66)\end{array}$ & 67.7 & - & - & 26.47 \\
\hline Cen et al. (2020) & Cen & $\begin{array}{l}2020-06- \\
08\end{array}$ & China & 1,007 & Hospital & $\begin{array}{l}61(49- \\
68)\end{array}$ & 51.0 & - & - & 8.74 \\
\hline $\begin{array}{l}\text { Houlihan et al. } \\
(2020)\end{array}$ & Houlihan & $\begin{array}{l}2020-06- \\
09\end{array}$ & UK & 200 & Community & $\begin{array}{l}34(29- \\
44)\end{array}$ & 61.0 & 11.0 & 16.50 & - \\
\hline Lan et al. (2020) & Lan & $\begin{array}{l}2020-06- \\
09\end{array}$ & USA & 104 & Community & $\begin{array}{l}49^{\wedge} \\
(34-63)\end{array}$ & 47.1 & - & - & 24.04 \\
\hline $\begin{array}{l}\text { Russell et al. } \\
\text { (2020) }\end{array}$ & Russell & $\begin{array}{l}2020-06- \\
09\end{array}$ & UK & 156 & $\begin{array}{l}\text { Community } \\
\text { and } \\
\text { Hospital }\end{array}$ & $\begin{array}{l}65.18^{\wedge} \\
(50-79)\end{array}$ & 42.3 & 7.1 & 25.00 & - \\
\hline Veras et al. (2020) & Veras & $\begin{array}{l}2020-06- \\
09\end{array}$ & Brazil & 32 & Hospital & $\begin{array}{l}58.9^{\wedge} \\
(40-77)\end{array}$ & 47.0 & - & - & 25.00 \\
\hline Rossi et al. (2020) & Rossi & $\begin{array}{l}2020-06- \\
09\end{array}$ & France & 246 & Hospital & $\begin{array}{l}68^{\wedge} \\
(53-83)\end{array}$ & 39.0 & - & - & 25.20 \\
\hline $\begin{array}{l}\text { Martin-Jimenez et } \\
\text { al. (2020) }\end{array}$ & Martin-Jiminez & $\begin{array}{l}2020-06- \\
09\end{array}$ & Spain & 339 & Hospital & $\begin{array}{l}81.6 \\
(72-87)\end{array}$ & 39.5 & - & - & 30.68 \\
\hline Rajter et al. (2020) & Rajter & $\begin{array}{l}2020-06- \\
10\end{array}$ & USA & 280 & Hospital & $\begin{array}{l}59.6^{\wedge} \\
(41-77)\end{array}$ & 45.5 & 5.7 & 10.71 & - \\
\hline Zhou et al. (2020) & Zhou, He & $\begin{array}{l}2020-06- \\
10\end{array}$ & China & 238 & Hospital & $\begin{array}{l}55.5 \\
(35-67)\end{array}$ & 57.0 & 2.9 & - & - \\
\hline $\begin{array}{l}\text { Woolford et al. } \\
(2020)\end{array}$ & Woolford & $\begin{array}{l}2020-06- \\
11\end{array}$ & UK & 4,510 & $\begin{array}{l}\text { Community } \\
\text { and } \\
\text { Hospital }\end{array}$ & $\begin{array}{l}70.5 \\
\text { (NA) }\end{array}$ & 51.2 & 13.0 & 38.12 & - \\
\hline $\begin{array}{l}\text { Hultcrantz et al. } \\
(2020)\end{array}$ & Hultcrantz & $\begin{array}{l}2020-06- \\
11\end{array}$ & USA & 127 & $\begin{array}{l}\text { Community } \\
\text { and } \\
\text { Hospital }\end{array}$ & $\begin{array}{l}68(41- \\
91)\end{array}$ & 46.0 & - & - & 26.77 \\
\hline $\begin{array}{l}\text { Hernández- } \\
\text { Garduño (2020) }\end{array}$ & $\begin{array}{l}\text { Hernandez, } \\
\text { Garduno }\end{array}$ & $\begin{array}{l}2020-06- \\
11\end{array}$ & Mexico & 32,583 & $\begin{array}{l}\text { Community } \\
\text { and } \\
\text { Hospital }\end{array}$ & $\begin{array}{l}45(34- \\
56)\end{array}$ & 48.7 & - & - & 11.02 \\
\hline Sterlin et al. (2020) & Sterlin & $\begin{array}{l}2020-06- \\
11\end{array}$ & France & 135 & Hospital & $\begin{array}{l}61(50- \\
72)\end{array}$ & 41.0 & 3.7 & 38.52 & - \\
\hline
\end{tabular}




\begin{tabular}{|c|c|c|c|c|c|c|c|c|c|c|c|}
\hline $\begin{array}{l}\text { Maraschini et al. } \\
(2020)\end{array}$ & Maraschini & $\begin{array}{l}2020-06- \\
12\end{array}$ & Italy & 146 & Hospital & $\begin{array}{l}32.5^{\wedge} \\
(27-38)\end{array}$ & 100.0 & - & 9.59 & - & 80. \\
\hline Wang et al. (2020) & Wang, Zhong & $\begin{array}{l}2020-06- \\
12\end{array}$ & USA & 7,592 & $\begin{array}{l}\text { Community } \\
\text { and } \\
\text { Hospital }\end{array}$ & NA & 45.1 & 3.6 & 17.08 & - & 51. \\
\hline $\begin{array}{l}\text { McQueenie et al. } \\
(2020)\end{array}$ & McQueenie & $\begin{array}{l}2020-06- \\
12\end{array}$ & UK & 428,199 & $\begin{array}{l}\text { Community } \\
\text { and } \\
\text { Hospital }\end{array}$ & NA & 54.9 & - & - & 44.36 & 55. \\
\hline Miyara et al. (2020) & Miyara & $\begin{array}{l}2020-06- \\
12\end{array}$ & France & 479 & $\begin{array}{l}\text { Community } \\
\text { and } \\
\text { Hospital }\end{array}$ & NA & 44.7 & 6.7 & 31.60 & - & 59. \\
\hline Apea et al. (2021) & Apea & $\begin{array}{l}2020-06- \\
12\end{array}$ & UK & 1,737 & Hospital & $\begin{array}{l}63.4^{\wedge} \\
\text { (NA) }\end{array}$ & 30.4 & - & - & 9.96 & - \\
\hline $\begin{array}{l}\text { Garassino et al. } \\
(2020)\end{array}$ & Garassino & $\begin{array}{l}2020-06- \\
12\end{array}$ & Multiple & 200 & $\begin{array}{l}\text { Community } \\
\text { and } \\
\text { Hospital }\end{array}$ & $\begin{array}{l}68 \\
(61.8- \\
75)\end{array}$ & 30.0 & 24.0 & 55.50 & - & 18. \\
\hline Zeng et al. (2020) & Zeng & $\begin{array}{l}2020-06- \\
16\end{array}$ & China & 1,031 & Hospital & $\begin{array}{l}60.3^{\wedge} \\
(46-74)\end{array}$ & 47.8 & - & - & 10.18 & - \\
\hline $\begin{array}{l}\text { Suleyman et al. } \\
(2020)\end{array}$ & Suleyman & $\begin{array}{l}2020-06- \\
16\end{array}$ & USA & 463 & Hospital & $\begin{array}{l}57.5^{\wedge} \\
(40-74)\end{array}$ & 55.9 & - & - & 34.56 & - \\
\hline Chen et al. (2020) & Chen, Yu & $\begin{array}{l}2020-06- \\
16\end{array}$ & China & 1,859 & Hospital & $\begin{array}{l}59(45- \\
68)\end{array}$ & 50.0 & 2.4 & 3.55 & - & 94. \\
\hline Kibler et al. (2020) & Kibler & $\begin{array}{l}2020-06- \\
16\end{array}$ & France & 702 & $\begin{array}{l}\text { Community } \\
\text { and } \\
\text { Hospital }\end{array}$ & $\begin{array}{l}82^{\wedge} \\
(75-88)\end{array}$ & 56.0 & 3.7 & - & - & - \\
\hline $\begin{array}{l}\text { Olivares et al. } \\
(2020)\end{array}$ & Olivares & $\begin{array}{l}2020-06- \\
16\end{array}$ & Chile & 21 & Hospital & $\begin{array}{l}61^{\wedge} \\
(26-85)\end{array}$ & 76.2 & - & - & 9.52 & - \\
\hline $\begin{array}{l}\text { Elezkurtaj et al. } \\
(2020)\end{array}$ & Elezkurtaj & $\begin{array}{l}2020-06- \\
17\end{array}$ & Germany & 26 & Hospital & $\begin{array}{l}70 \\
(61.8- \\
78.3)\end{array}$ & 34.6 & - & - & 19.23 & - \\
\hline Zuo et al. (2020) & Zuo, Estes & $\begin{array}{l}2020-06- \\
17\end{array}$ & China & 172 & Hospital & $\begin{array}{l}61^{\wedge} \\
(25-95)\end{array}$ & 44.0 & - & - & 26.16 & - \\
\hline Killerby (2020) & Killerby & $\begin{array}{l}2020-06- \\
17\end{array}$ & USA & 531 & $\begin{array}{l}\text { Community } \\
\text { and } \\
\text { Hospital }\end{array}$ & $\begin{array}{l}51.6 \\
(38-62)\end{array}$ & 57.1 & - & - & 17.14 & 71. \\
\hline Gu et al. (2020) & Gu & $\begin{array}{l}2020-06- \\
18\end{array}$ & USA & 5,698 & $\begin{array}{l}\text { Community } \\
\text { and } \\
\text { Hospital }\end{array}$ & $\begin{array}{l}47^{\wedge} \\
(26-67)\end{array}$ & 62.0 & 7.0 & 24.68 & - & 50. \\
\hline Wei et al. (2020) & Wei & $\begin{array}{l}2020-06- \\
18\end{array}$ & USA & 147 & Hospital & $\begin{array}{l}52^{\wedge} \\
(34-70)\end{array}$ & 41.0 & 14.3 & - & - & - \\
\hline $\begin{array}{l}\text { Crovetto et al. } \\
(2020)\end{array}$ & Crovetto & $\begin{array}{l}2020-06- \\
19\end{array}$ & Spain & 874 & $\begin{array}{l}\text { Community } \\
\text { and } \\
\text { Hospital }\end{array}$ & $\begin{array}{l}33.7^{\wedge} \\
(28-38)\end{array}$ & 100.0 & 1.1 & - & - & - \\
\hline $\begin{array}{l}\text { Govind et al. } \\
(2020)\end{array}$ & Govind & $\begin{array}{l}2020-06- \\
20\end{array}$ & UK & 6,309 & $\begin{array}{l}\text { Community } \\
\text { and } \\
\text { Hospital }\end{array}$ & $\begin{array}{l}46.5^{\wedge} \\
(31-61)\end{array}$ & 38.3 & 66.3 & 26.77 & - & 5.5 \\
\hline $\begin{array}{l}\text { Sisó-Almirall et al. } \\
(2020)\end{array}$ & Siso-Almirall & $\begin{array}{l}2020-06- \\
20\end{array}$ & Spain & 322 & $\begin{array}{l}\text { Community } \\
\text { and } \\
\text { Hospital }\end{array}$ & $\begin{array}{l}56.7^{\wedge} \\
(38-74)\end{array}$ & 50.0 & - & - & 25.16 & - \\
\hline Salton et al. (2020) & Salton & $\begin{array}{l}2020-06- \\
20\end{array}$ & Italy & 173 & Hospital & $\begin{array}{l}64.4^{\wedge} \\
(\mathrm{NA})\end{array}$ & 34.9 & - & - & 29.48 & - \\
\hline Duan et al. (2020) & Duan & $\begin{array}{l}2020-06- \\
22\end{array}$ & China & 616 & Hospital & $\begin{array}{l}64(53- \\
70)\end{array}$ & 57.5 & 3.7 & - & - & - \\
\hline
\end{tabular}




\begin{tabular}{|c|c|c|c|c|c|c|c|c|c|c|c|}
\hline Lenka et al. (2020) & Lenka & $\begin{array}{l}2020-06- \\
22\end{array}$ & USA & 32 & Hospital & $\begin{array}{l}62.2^{\wedge} \\
(51-73)\end{array}$ & 37.5 & - & - & 50.00 & - \\
\hline $\begin{array}{l}\text { Fisman et al. } \\
(2020)\end{array}$ & Fisman & $\begin{array}{l}2020-06- \\
23\end{array}$ & Canada & 21,922 & $\begin{array}{l}\text { Community } \\
\text { and } \\
\text { Hospital }\end{array}$ & NA & 57.0 & - & - & 2.35 & - \\
\hline $\begin{array}{l}\text { Madariaga et al. } \\
(2020)\end{array}$ & Madariaga & $\begin{array}{l}2020-06- \\
23\end{array}$ & USA & 103 & $\begin{array}{l}\text { Community } \\
\text { and } \\
\text { Hospital }\end{array}$ & $\begin{array}{l}41.8^{\wedge} \\
(27-55)\end{array}$ & 48.5 & - & - & 25.24 & 74. \\
\hline Jin et al. (2020) & Jin, Gu & $\begin{array}{l}2020-06- \\
25\end{array}$ & China & 6 & Hospital & $\begin{array}{l}60.5^{\wedge} \\
(51-75)\end{array}$ & 33.3 & 33.3 & - & - & - \\
\hline Mendy et al. (2020) & Mendy & $\begin{array}{l}2020-06- \\
27\end{array}$ & USA & 689 & $\begin{array}{l}\text { Community } \\
\text { and } \\
\text { Hospital }\end{array}$ & $\begin{array}{l}49.5 \\
(35.2- \\
67.5)\end{array}$ & 47.0 & - & - & 24.67 & - \\
\hline Sigel et al. (2020) & Sigel & $\begin{array}{l}2020-06- \\
28\end{array}$ & USA & 493 & Hospital & $\begin{array}{l}60(55- \\
67)\end{array}$ & 24.1 & - & - & 28.60 & - \\
\hline Souza et al. (2020) & de Souza & $\begin{array}{l}2020-06- \\
28\end{array}$ & Brazil & 8,443 & Hospital & NA & 53.0 & - & - & 1.68 & - \\
\hline $\begin{array}{l}\text { Nguyen et al. } \\
(2020)\end{array}$ & Nguyen & $\begin{array}{l}2020-06- \\
29\end{array}$ & USA & 689 & $\begin{array}{l}\text { Community } \\
\text { and } \\
\text { Hospital }\end{array}$ & $\begin{array}{l}55(40- \\
68)\end{array}$ & 57.0 & - & - & 24.82 & - \\
\hline Melo et al. (2020) & de Melo & $\begin{array}{l}2020-06- \\
29\end{array}$ & Brazil & 181 & Hospital & $\begin{array}{l}55.3^{\wedge} \\
(34-76)\end{array}$ & 60.8 & 9.9 & 12.15 & - & 38. \\
\hline $\begin{array}{l}\text { Auvinen et al. } \\
(2020)\end{array}$ & Auvinen & $\begin{array}{l}2020-06- \\
29\end{array}$ & Finland & 61 & Hospital & $\begin{array}{l}53(41- \\
67)\end{array}$ & 36.0 & 18.0 & 27.87 & - & 54. \\
\hline $\begin{array}{l}\text { Magleby et al. } \\
(2020)\end{array}$ & Magleby & $\begin{array}{l}2020-06- \\
30\end{array}$ & USA & 678 & Hospital & $\begin{array}{l}68(50- \\
81)\end{array}$ & 38.9 & - & - & 28.61 & - \\
\hline Hewitt et al. (2020) & Hewitt & $\begin{array}{l}2020-06- \\
30\end{array}$ & Multiple & 1,564 & Hospital & $\begin{array}{l}74(61- \\
83)\end{array}$ & 42.3 & 7.7 & 38.55 & - & 52. \\
\hline $\begin{array}{l}\text { Mohamud et al. } \\
(2020)\end{array}$ & Mohamud & $\begin{array}{l}2020-07- \\
02\end{array}$ & USA & 6 & Hospital & $\begin{array}{l}65.8^{\wedge} \\
(55-78)\end{array}$ & 16.7 & - & - & 16.67 & - \\
\hline $\begin{array}{l}\text { Trubiano et al. } \\
(2020)\end{array}$ & Trubiano & $\begin{array}{l}2020-07- \\
02\end{array}$ & Australia & 2,935 & $\begin{array}{l}\text { Community } \\
\text { and } \\
\text { Hospital }\end{array}$ & $\begin{array}{l}39(29- \\
53)\end{array}$ & 63.5 & - & - & 8.82 & - \\
\hline Patel et al. (2020) & Patel & $\begin{array}{l}2020-07- \\
03\end{array}$ & USA & 129 & Hospital & $\begin{array}{l}60.8^{\wedge} \\
(47-74)\end{array}$ & 45.0 & 37.2 & - & - & - \\
\hline $\begin{array}{l}\text { Merzon et al. } \\
(2020)\end{array}$ & Merzon & $\begin{array}{l}2020-07- \\
03\end{array}$ & Israel & 7,807 & $\begin{array}{l}\text { Community } \\
\text { and } \\
\text { Hospital }\end{array}$ & $\begin{array}{l}46.2^{\wedge} \\
(\mathrm{NA})\end{array}$ & 58.6 & - & - & 16.18 & - \\
\hline $\begin{array}{l}\text { Bello-Chavolla et } \\
\text { al. }(2020)\end{array}$ & $\begin{array}{l}\text { Bello-Chavolla, } \\
\text { Antonio-Villa }\end{array}$ & $\begin{array}{l}2020-07- \\
04\end{array}$ & Mexico & 60,121 & $\begin{array}{l}\text { Community } \\
\text { and } \\
\text { Hospital }\end{array}$ & $\begin{array}{l}45.5^{\wedge} \\
(29-61)\end{array}$ & 47.0 & - & - & 10.48 & - \\
\hline $\begin{array}{l}\text { Zacharioudakis et } \\
\text { al. (2020) }\end{array}$ & Zacharioudakis & $\begin{array}{l}2020-07- \\
04\end{array}$ & USA & 314 & Hospital & $\begin{array}{l}64(54- \\
72)\end{array}$ & 34.7 & - & - & 22.78 & - \\
\hline $\begin{array}{l}\text { Antonio-Villa et al. } \\
(2020)\end{array}$ & Antonio-Villa & $\begin{array}{l}2020-07- \\
04\end{array}$ & Mexico & 34,263 & $\begin{array}{l}\text { Community } \\
\text { and } \\
\text { Hospital }\end{array}$ & $\begin{array}{l}40^{\wedge} \\
(29-50)\end{array}$ & 62.9 & 9.7 & - & - & - \\
\hline $\begin{array}{l}\text { Kimmig et al. } \\
(2020)\end{array}$ & Kimmig & $\begin{array}{l}2020-07- \\
06\end{array}$ & USA & 111 & Hospital & $\begin{array}{l}63^{\wedge} \\
(48-78)\end{array}$ & 44.1 & 7.2 & 36.04 & - & 56. \\
\hline Senkal (2020) & Senkal & $\begin{array}{l}2020-07- \\
07\end{array}$ & Turkey & 611 & Hospital & $\begin{array}{l}57^{\wedge} \\
(18-98)\end{array}$ & 40.6 & 11.3 & - & - & - \\
\hline Xie et al. (2020) & Xie & $\begin{array}{l}2020-07- \\
07\end{array}$ & China & 619 & Hospital & NA & 52.0 & - & - & 8.24 & - \\
\hline
\end{tabular}




\begin{tabular}{|c|c|c|c|c|c|c|c|c|c|c|}
\hline $\begin{array}{l}\text { Elmunzer et al. } \\
(2020)\end{array}$ & Elmunzer & $\begin{array}{l}2020-07- \\
09\end{array}$ & Multiple & 1,992 & Hospital & $\begin{array}{l}60^{\wedge} \\
(43-76)\end{array}$ & 43.0 & 6.3 & 28.56 & - \\
\hline $\begin{array}{l}\text { Alizadehsani et al. } \\
(2020)\end{array}$ & Alizadehsani & $\begin{array}{l}2020-07- \\
09\end{array}$ & Iran & 319 & Hospital & $\begin{array}{l}45.48^{\wedge} \\
(26-63)\end{array}$ & 55.5 & - & - & 0.31 \\
\hline $\begin{array}{l}\text { Maucourant et al. } \\
(2020)\end{array}$ & Maucourant & $\begin{array}{l}2020-07- \\
10\end{array}$ & Sweden & 27 & Hospital & $\begin{array}{l}57(18- \\
78)\end{array}$ & 22.2 & 11.1 & 25.93 & - \\
\hline Fan et al. (2020) & Fan & $\begin{array}{l}2020-07- \\
11\end{array}$ & UK & 1,425 & $\begin{array}{l}\text { Community } \\
\text { and } \\
\text { Hospital }\end{array}$ & NA & 46.7 & 12.2 & 40.07 & - \\
\hline Shi et al. (2020) & Shi, Resurreccion & $\begin{array}{l}2020-07- \\
11\end{array}$ & UK & 1,521 & $\begin{array}{l}\text { Community } \\
\text { and } \\
\text { Hospital }\end{array}$ & $\begin{array}{l}61.5^{\wedge} \\
(57- \\
66.8)\end{array}$ & 45.9 & - & - & 54.90 \\
\hline $\begin{array}{l}\text { REACT Study } \\
\text { Investigators et al. } \\
(2020)\end{array}$ & Riley & $\begin{array}{l}2020-07- \\
11\end{array}$ & UK & 120,620 & $\begin{array}{l}\text { Community } \\
\text { and } \\
\text { Hospital }\end{array}$ & NA & 54.0 & 2.2 & - & - \\
\hline $\begin{array}{l}\text { Hippisley-Cox et } \\
\text { al. (2020) }\end{array}$ & Hippisley-Cox & $\begin{array}{l}2020-07- \\
13\end{array}$ & UK & $8,275,949$ & $\begin{array}{l}\text { Community } \\
\text { and } \\
\text { Hospital }\end{array}$ & $\begin{array}{l}48.5^{\wedge} \\
(30-66)\end{array}$ & 50.3 & 17.2 & 21.44 & - \\
\hline Zhang et al. (2020) & Zhang, Cao & $\begin{array}{l}2020-07- \\
14\end{array}$ & China & 289 & Hospital & $\begin{array}{l}57(22- \\
88)\end{array}$ & 46.6 & 3.5 & 6.23 & - \\
\hline Eiros et al. (2020) & Eiros & $\begin{array}{l}2020-07- \\
14\end{array}$ & Spain & 139 & $\begin{array}{l}\text { Community } \\
\text { and } \\
\text { Hospital }\end{array}$ & $\begin{array}{l}52(41- \\
57)\end{array}$ & 72.0 & 4.3 & 50.36 & - \\
\hline $\begin{array}{l}\text { Marcos et al. } \\
(2020)\end{array}$ & Marcos & $\begin{array}{l}2020-07- \\
14\end{array}$ & Spain & 918 & Hospital & $\begin{array}{l}72.8^{\wedge} \\
(58-87)\end{array}$ & 42.2 & 6.1 & - & 15.25 \\
\hline $\begin{array}{l}\text { Hoertel et al. } \\
(2020)\end{array}$ & $\begin{array}{l}\text { Hoertel, Sanchez, } \\
\text { Rico }\end{array}$ & $\begin{array}{l}2020-07- \\
14\end{array}$ & France & 7,345 & Hospital & NA & 49.3 & 8.5 & - & - \\
\hline Shi et al. (2020) & Shi, Zuo & $\begin{array}{l}2020-07- \\
15\end{array}$ & USA & 172 & Hospital & $\begin{array}{l}61.48^{\wedge} \\
(25-96)\end{array}$ & 44.0 & - & - & 26.16 \\
\hline $\begin{array}{l}\text { Hussein et al. } \\
(2020)\end{array}$ & Hussein & $\begin{array}{l}2020-07- \\
15\end{array}$ & USA & 502 & Hospital & $\begin{array}{l}60.9^{\wedge} \\
(45-76)\end{array}$ & 52.0 & 9.0 & 22.11 & - \\
\hline Bian et al. (2020) & Bian & $\begin{array}{l}2020-07- \\
15\end{array}$ & China & 28 & Hospital & $\begin{array}{l}56^{\wedge} \\
(42-67)\end{array}$ & 42.9 & 7.1 & - & - \\
\hline Zhan et al. (2020) & Zhan & $\begin{array}{l}2020-07- \\
16\end{array}$ & China & 75 & Hospital & $\begin{array}{l}57(25- \\
75)\end{array}$ & 48.0 & - & - & 12.00 \\
\hline $\begin{array}{l}\text { Omrani et al. } \\
(2020)\end{array}$ & Omrani & $\begin{array}{l}2020-07- \\
16\end{array}$ & Qatar & 1,409 & $\begin{array}{l}\text { Community } \\
\text { and } \\
\text { Hospital }\end{array}$ & $\begin{array}{l}39(30- \\
50)\end{array}$ & 17.2 & - & - & 9.23 \\
\hline Gupta et al. (2020) & Gupta & $\begin{array}{l}2020-07- \\
16\end{array}$ & USA & 496 & Hospital & $\begin{array}{l}70(60- \\
78)\end{array}$ & 46.0 & - & - & 7.26 \\
\hline $\begin{array}{l}\text { Soares et al. } \\
(2020)\end{array}$ & Soares & $\begin{array}{l}2020-07- \\
16\end{array}$ & Brazil & 10,713 & $\begin{array}{l}\text { Community } \\
\text { and } \\
\text { Hospital }\end{array}$ & NA & 55.0 & 2.0 & - & - \\
\hline $\begin{array}{l}\text { Abolghasemi et al. } \\
(2020)\end{array}$ & Abolghasemi & $\begin{array}{l}2020-07- \\
17\end{array}$ & Iran & 24 & Hospital & $\begin{array}{l}49^{\wedge} \\
(29-64)\end{array}$ & 37.5 & - & - & 4.17 \\
\hline $\begin{array}{l}\text { Merkely et al. } \\
(2020)\end{array}$ & Merkely & $\begin{array}{l}2020-07- \\
17\end{array}$ & Hungary & 10,474 & Community & $\begin{array}{l}48.7^{\wedge} \\
(30-66)\end{array}$ & 53.6 & 28.0 & 20.46 & - \\
\hline Fox et al. (2020) & Fox & $\begin{array}{l}2020-07- \\
17\end{array}$ & UK & 55 & $\begin{array}{l}\text { Community } \\
\text { and } \\
\text { Hospital }\end{array}$ & $\begin{array}{l}63(23- \\
88)\end{array}$ & 31.0 & 1.8 & 10.91 & - \\
\hline $\begin{array}{l}\text { Pandolfi et al. } \\
(2020)\end{array}$ & Pandolfi & $\begin{array}{l}2020-07- \\
17\end{array}$ & Italy & 33 & Hospital & $\begin{array}{l}62(52- \\
65)\end{array}$ & 21.1 & 3.0 & 24.24 & - \\
\hline Girardeau et al. & & 2020-07- & & & & $30129-$ & & & & \\
\hline
\end{tabular}

59. 


\begin{tabular}{|c|c|c|c|c|c|c|c|c|c|c|}
\hline$(2020)$ & Girardeau & 17 & France & 10 & Community & $33)^{1-}$ & 50.0 & 40.0 & 10.00 & - \\
\hline $\begin{array}{l}\text { Kurashima et al. } \\
\text { (2020) }\end{array}$ & Kurashima & $\begin{array}{l}2020-07- \\
17\end{array}$ & Japan & 53 & Hospital & $\begin{array}{l}62.9^{\wedge} \\
(49-76)\end{array}$ & 35.8 & - & - & 50.94 \\
\hline $\begin{array}{l}\text { McGrail and } \\
\text { Edwards (2020) }\end{array}$ & Edwards & $\begin{array}{l}2020-07- \\
19\end{array}$ & USA & 209 & Hospital & $\begin{array}{l}62.5 \\
\text { (NA) }\end{array}$ & 38.8 & - & - & 18.66 \\
\hline $\begin{array}{l}\text { Martinez- } \\
\text { Resendez et al. } \\
(2020)\end{array}$ & $\begin{array}{l}\text { Martinez- } \\
\text { Resendez }\end{array}$ & $\begin{array}{l}2020-07- \\
20\end{array}$ & Mexico & 8 & Hospital & $\begin{array}{l}57(48- \\
69)\end{array}$ & 25.0 & - & - & 12.50 \\
\hline $\begin{array}{l}\text { Hoertel et al. } \\
(2020)\end{array}$ & Hoertel & $\begin{array}{l}2020-07- \\
20\end{array}$ & France & 12,612 & Hospital & $\begin{array}{l}58.7^{\wedge} \\
(39-77)\end{array}$ & 49.6 & - & - & 9.28 \\
\hline Wang et al. (2020) & Wang, Shu & $\begin{array}{l}2020-07- \\
20\end{array}$ & China & 59 & Hospital & $\begin{array}{l}67.4^{\wedge} \\
(56-78)\end{array}$ & 35.6 & - & - & 15.25 \\
\hline $\begin{array}{l}\text { Bernaola et al. } \\
(2020)\end{array}$ & Bernaola & $\begin{array}{l}2020-07- \\
21\end{array}$ & Spain & 1,645 & Hospital & NA & 38.5 & 2.5 & 10.88 & - \\
\hline $\begin{array}{l}\text { Schneeweiss et al. } \\
(2020)\end{array}$ & Schneeweiss & $\begin{array}{l}2020-07- \\
22\end{array}$ & USA & 24,313 & $\begin{array}{l}\text { Community } \\
\text { and } \\
\text { Hospital }\end{array}$ & $\begin{array}{l}67^{\wedge} \\
(53-80)\end{array}$ & 53.0 & - & - & 2.88 \\
\hline $\begin{array}{l}\text { Concha-Mejia and } \\
\text { Rincon-Sanchez } \\
(2020)\end{array}$ & Mejia & $\begin{array}{l}2020-07- \\
24\end{array}$ & Colombia & 72 & $\begin{array}{l}\text { Community } \\
\text { and } \\
\text { Hospital }\end{array}$ & $\begin{array}{l}46(28- \\
64)\end{array}$ & 47.0 & 8.3 & 11.11 & - \\
\hline $\begin{array}{l}\text { Izquierdo et al. } \\
(2020)\end{array}$ & Izquierdo & $\begin{array}{l}2020-07- \\
24\end{array}$ & Spain & 71,192 & $\begin{array}{l}\text { Community } \\
\text { and } \\
\text { Hospital }\end{array}$ & $\begin{array}{l}42^{\wedge} \\
(18-66)\end{array}$ & 59.0 & 10.0 & - & - \\
\hline $\begin{array}{l}\text { Santos et al. } \\
(2020)\end{array}$ & Santos & $\begin{array}{l}2020-07- \\
25\end{array}$ & USA & 23 & $\begin{array}{l}\text { Community } \\
\text { and } \\
\text { Hospital }\end{array}$ & NA & - & - & - & 8.70 \\
\hline Reiter et al. (2020) & Reiter & $\begin{array}{l}2020-07- \\
26\end{array}$ & Austria & 235 & Community & $\begin{array}{l}44.2^{\wedge} \\
(32-55)\end{array}$ & 70.0 & 22.6 & 22.55 & - \\
\hline Motta et al. (2020) & Motta & $\begin{array}{l}2020-07- \\
26\end{array}$ & USA & 374 & Hospital & $\begin{array}{l}64.7^{\wedge} \\
(46-82)\end{array}$ & 41.4 & - & - & 33.16 \\
\hline $\begin{array}{l}\text { Altamimi et al. } \\
(2020)\end{array}$ & Altamimi & $\begin{array}{l}2020-07- \\
27\end{array}$ & Qatar & 68 & Hospital & $\begin{array}{l}49^{\wedge} \\
(40-58)\end{array}$ & 2.0 & 16.2 & - & - \\
\hline $\begin{array}{l}\text { Thompson et al. } \\
(2020)\end{array}$ & Thompson & $\begin{array}{l}2020-07- \\
27\end{array}$ & UK & 470 & Hospital & $\begin{array}{l}71(57- \\
82)\end{array}$ & 46.0 & 14.0 & 27.23 & - \\
\hline $\begin{array}{l}\text { Zobairy et al. } \\
(2020)\end{array}$ & Zobairy & $\begin{array}{l}2020-07- \\
28\end{array}$ & Iran & 203 & $\begin{array}{l}\text { Community } \\
\text { and } \\
\text { Hospital }\end{array}$ & $\begin{array}{l}49.2^{\wedge} \\
(32-65)\end{array}$ & 44.8 & 5.9 & - & - \\
\hline Zhou et al. (2020) & Zhou, Sun & $\begin{array}{l}2020-07- \\
29\end{array}$ & China & 144 & Hospital & $\begin{array}{l}47(38- \\
56)\end{array}$ & 46.5 & 9.0 & - & - \\
\hline Kumar et al. (2020) & Kumar & $\begin{array}{l}2020-07- \\
29\end{array}$ & India & 91 & Hospital & $\begin{array}{l}47^{\wedge} \\
(41-52)\end{array}$ & 21.0 & 44.0 & - & - \\
\hline Qu et al. (2020) & Qu & $\begin{array}{l}2020-07- \\
29\end{array}$ & China & 246 & Hospital & $\begin{array}{l}53.6^{\wedge} \\
(38-68)\end{array}$ & 53.3 & 42.3 & - & - \\
\hline $\begin{array}{l}\text { Higuchi et al. } \\
\text { (2020) }\end{array}$ & Higuchi & $\begin{array}{l}2020-07- \\
30\end{array}$ & Japan & 57 & Hospital & $\begin{array}{l}52(35- \\
70)\end{array}$ & 43.9 & 12.3 & 29.82 & - \\
\hline Zhao et al. (2020) & Zhao, Chen & $\begin{array}{l}2020-07- \\
30\end{array}$ & USA & 641 & Hospital & $\begin{array}{l}60 \\
\text { (NA) }\end{array}$ & 40.1 & 21.7 & - & - \\
\hline Fond et al. (2020) & Fond & $\begin{array}{l}2020-07- \\
30\end{array}$ & France & 1,092 & Hospital & $\begin{array}{l}62.5 \\
(51-76)\end{array}$ & 45.7 & 11.4 & - & - \\
\hline Jun et al. (2020) & Jun & $\begin{array}{l}2020-08- \\
01\end{array}$ & USA & 3,086 & Hospital & $\begin{array}{l}66(56- \\
77)\end{array}$ & 40.9 & 3.7 & 21.32 & - \\
\hline Mnrshed et al & & วกวก-ก৪- & & & & $371.31-$ & & & & \\
\hline
\end{tabular}




\begin{tabular}{|c|c|c|c|c|c|c|c|c|c|c|c|}
\hline$(2020)$ & Morshed & 02 & Bangladesh & 103 & Community & $53)^{\prime \prime \prime}$ & 28.2 & 31.1 & - & - & - \\
\hline $\begin{array}{l}\text { Iversen et al. } \\
(2020)\end{array}$ & Iversen & $\begin{array}{l}2020-08- \\
03\end{array}$ & Denmark & 28,792 & $\begin{array}{l}\text { Community } \\
\text { and } \\
\text { Hospital }\end{array}$ & $\begin{array}{l}44.4^{\wedge} \\
(31-57)\end{array}$ & 78.9 & 16.0 & 6.52 & - & 76. \\
\hline $\begin{array}{l}\text { Ebinger et al. } \\
(2020)\end{array}$ & Ebinger & $\begin{array}{l}2020-08- \\
04\end{array}$ & USA & 6,062 & Community & $\begin{array}{l}41.5^{\wedge} \\
(29-53)\end{array}$ & 67.8 & 1.7 & - & - & - \\
\hline Hadi et al. (2020) & Hadi & $\begin{array}{l}2020-08- \\
05\end{array}$ & USA & 370 & $\begin{array}{l}\text { Community } \\
\text { and } \\
\text { Hospital }\end{array}$ & $\begin{array}{l}48.2^{\wedge} \\
(34-62)\end{array}$ & 29.5 & - & - & 15.14 & 84. \\
\hline Tao et al. (2020) & Tao & $\begin{array}{l}2020-08- \\
06\end{array}$ & China & 70 & Community & $\begin{array}{l}33.2^{\wedge} \\
(12-53)\end{array}$ & 48.6 & - & - & 15.71 & - \\
\hline Klang et al. (2020) & Klang, Soffer & $\begin{array}{l}2020-08- \\
09\end{array}$ & USA & 1,320 & Hospital & NA & 41.5 & - & - & 24.70 & - \\
\hline Zhou et al. (2021) & Zhou, Ma & $\begin{array}{l}2020-08- \\
10\end{array}$ & China & 429 & Hospital & $\begin{array}{l}58.3^{\wedge} \\
(42-74)\end{array}$ & 50.6 & - & - & 8.39 & - \\
\hline Altibi et al. (2020) & Altibi & $\begin{array}{l}2020-08- \\
11\end{array}$ & USA & 706 & Hospital & $\begin{array}{l}66.7^{\wedge} \\
(51-81)\end{array}$ & 43.0 & 4.0 & 37.25 & - & 58. \\
\hline $\begin{array}{l}\text { Izzi-Engbeaya et } \\
\text { al. (2020) }\end{array}$ & Izzi-Engbeaya & $\begin{array}{l}2020-08- \\
11\end{array}$ & UK & 889 & Hospital & $\begin{array}{l}65.8^{\wedge} \\
(48-83)\end{array}$ & 40.0 & - & - & 21.26 & 33. \\
\hline Rizzo et al. (2020) & Rizzo & $\begin{array}{l}2020-08- \\
11\end{array}$ & USA & 76,819 & Hospital & $\begin{array}{l}54(38- \\
67)\end{array}$ & 55.2 & 6.7 & 20.78 & - & 50. \\
\hline Jehi et al. (2020) & Jehi & $\begin{array}{l}2020-08- \\
11\end{array}$ & USA & 4,536 & $\begin{array}{l}\text { Community } \\
\text { and } \\
\text { Hospital }\end{array}$ & NA & - & 7.3 & 28.48 & - & 49. \\
\hline $\begin{array}{l}\text { Ouyang et al. } \\
(2020)\end{array}$ & Ouyang & $\begin{array}{l}2020-08- \\
14\end{array}$ & China & 217 & Hospital & $\begin{array}{l}46.5^{\wedge} \\
(30-62)\end{array}$ & 53.5 & 16.6 & - & - & - \\
\hline $\begin{array}{l}\text { Valenzuela et al. } \\
(2020)\end{array}$ & Valenzuela & $\begin{array}{l}2020-08- \\
14\end{array}$ & Chile & 29 & Hospital & $\begin{array}{l}56.9^{\wedge} \\
(43-70)\end{array}$ & 6.9 & 17.2 & - & - & - \\
\hline $\begin{array}{l}\text { Monteiro et al. } \\
(2020)\end{array}$ & Monteiro & $\begin{array}{l}2020-08- \\
14\end{array}$ & USA & 112 & Hospital & $\begin{array}{l}61(45- \\
74)\end{array}$ & 34.0 & 6.2 & 17.86 & - & 68. \\
\hline $\begin{array}{l}\text { Philipose et al. } \\
\text { (2020) }\end{array}$ & Philipose & $\begin{array}{l}2020-08- \\
14\end{array}$ & UK & 466 & Hospital & $\begin{array}{l}67(6- \\
97)\end{array}$ & 41.8 & 6.0 & 73.18 & - & 16. \\
\hline $\begin{array}{l}\text { Weerahandi et al. } \\
(2020)\end{array}$ & Weerahandi & $\begin{array}{l}2020-08- \\
14\end{array}$ & USA & 394 & Community & $\begin{array}{l}63(55- \\
70)\end{array}$ & 37.0 & 5.3 & 25.89 & - & 55. \\
\hline $\begin{array}{l}\text { Parra-Bracamonte } \\
\text { et al. }(2020)\end{array}$ & Parra-Bracamonte & $\begin{array}{l}2020-08- \\
14\end{array}$ & Mexico & 331,298 & $\begin{array}{l}\text { Community } \\
\text { and } \\
\text { Hospital }\end{array}$ & $\begin{array}{l}44(33- \\
56)\end{array}$ & 46.2 & - & - & 7.39 & - \\
\hline Peters et al. (2020) & Peters & $\begin{array}{l}2020-08- \\
15\end{array}$ & Netherlands & 1,893 & Hospital & $\begin{array}{l}66.8^{\wedge} \\
(52-81)\end{array}$ & 39.4 & 4.9 & - & - & - \\
\hline Islam et al. (2020) & Islam & $\begin{array}{l}2020-08- \\
18\end{array}$ & Bangladesh & 1,016 & $\begin{array}{l}\text { Community } \\
\text { and } \\
\text { Hospital }\end{array}$ & $\begin{array}{l}37(28- \\
49)\end{array}$ & 35.9 & 18.2 & - & - & - \\
\hline Chand et al. (2020) & Chand & $\begin{array}{l}2020-08- \\
19\end{array}$ & USA & 300 & Hospital & $\begin{array}{l}58.2^{\wedge} \\
(45-70)\end{array}$ & 39.3 & 22.3 & - & - & - \\
\hline Aksu et al. (2020) & Aksu & $\begin{array}{l}2020-08- \\
19\end{array}$ & Turkey & 123 & $\begin{array}{l}\text { Community } \\
\text { and } \\
\text { Hospital }\end{array}$ & $\begin{array}{l}49.7^{\wedge} \\
(36-63)\end{array}$ & 33.3 & 11.4 & - & - & - \\
\hline
\end{tabular}




\begin{tabular}{|c|c|c|c|c|c|c|c|c|c|c|c|}
\hline \multirow{2}{*}{\multicolumn{12}{|c|}{$1+-\cdots$}} \\
\hline & & & & & & & & & & & \\
\hline Ward et al. (2020) & Ward & $\begin{array}{l}2020-08- \\
21\end{array}$ & UK & 99,908 & Community & NA & 56.1 & 10.6 & - & - & - \\
\hline $\begin{array}{l}\text { Salerno et al. } \\
(2020)\end{array}$ & Salerno & $\begin{array}{l}2020-08- \\
22\end{array}$ & USA & 15,920 & Hospital & $\begin{array}{l}49(30- \\
65)\end{array}$ & 57.0 & - & - & 36.78 & 55 \\
\hline $\begin{array}{l}\text { Rashid et al. } \\
(2020)\end{array}$ & Rashid & $\begin{array}{l}2020-08- \\
22\end{array}$ & UK & 517 & Hospital & $\begin{array}{l}72.8^{\wedge} \\
(59-86)\end{array}$ & 31.9 & 9.9 & 29.01 & - & 29 \\
\hline Pan et al. (2020) & Pan & $\begin{array}{l}2020-08- \\
22\end{array}$ & USA & 12,084 & $\begin{array}{l}\text { Community } \\
\text { and } \\
\text { Hospital }\end{array}$ & $\begin{array}{l}45.5^{\wedge} \\
(27-63)\end{array}$ & 54.3 & - & - & 17.51 & - \\
\hline $\begin{array}{l}\text { Fillmore et al. } \\
(2020)\end{array}$ & Fillmore & $\begin{array}{l}2020-08- \\
24\end{array}$ & USA & 22,914 & $\begin{array}{l}\text { Community } \\
\text { and } \\
\text { Hospital }\end{array}$ & NA & - & 37.5 & 40.65 & - & 15 \\
\hline Zhou et al. (2020) & Zhou, Qin & $\begin{array}{l}2020-08- \\
25\end{array}$ & China & 51 & Hospital & $\begin{array}{l}57.37^{\wedge} \\
(42-72)\end{array}$ & 29.4 & - & - & 78.43 & 21 \\
\hline $\begin{array}{l}\text { lbrahim et al. } \\
(2020)\end{array}$ & Ibrahim & $\begin{array}{l}2020-08- \\
27\end{array}$ & USA & 38 & Hospital & $\begin{array}{l}63^{\wedge} \\
(51-75)\end{array}$ & 47.0 & 10.5 & - & - & - \\
\hline $\begin{array}{l}\text { Oliveira et al. } \\
(2020)\end{array}$ & Oliveira & $\begin{array}{l}2020-08- \\
31\end{array}$ & USA & 131 & Hospital & $\begin{array}{l}61 \\
(49.5- \\
71.5)\end{array}$ & 64.9 & - & - & 17.56 & 26 \\
\hline Yoo et al. (2020) & Yoo & $\begin{array}{l}2020-08- \\
31\end{array}$ & USA & 4,840 & Hospital & $\begin{array}{l}66.4 \\
(54.9- \\
77.8)\end{array}$ & 43.5 & 4.4 & 21.38 & - & 53 \\
\hline Zhan et al. (2020) & Zhan, Liu & $\begin{array}{l}2020-08- \\
31\end{array}$ & China & 405 & Hospital & $\begin{array}{l}56^{\wedge} \\
(17-95)\end{array}$ & 54.1 & - & - & 11.36 & 88 \\
\hline $\begin{array}{l}\text { Villar-Garcia et al. } \\
(2020)\end{array}$ & Vilar-Garcia & $\begin{array}{l}2020-09- \\
01\end{array}$ & Spain & $7,699,568$ & $\begin{array}{l}\text { Community } \\
\text { and } \\
\text { Hospital }\end{array}$ & $\begin{array}{l}43(24- \\
59)\end{array}$ & 50.9 & 17.1 & - & - & - \\
\hline $\begin{array}{l}\text { Ibarra-Nava et al. } \\
(2020)\end{array}$ & Ibarra-Nava & $\begin{array}{l}2020-09- \\
01\end{array}$ & Mexico & 416,546 & $\begin{array}{l}\text { Community } \\
\text { and } \\
\text { Hospital }\end{array}$ & NA & 46.9 & 7.4 & - & - & - \\
\hline $\begin{array}{l}\text { Rubio-Rivas et al. } \\
\text { (2020) }\end{array}$ & Rubio-Rivas & $\begin{array}{l}2020-09- \\
01\end{array}$ & Spain & 186 & Hospital & $\begin{array}{l}64.3^{\wedge} \\
(51-77)\end{array}$ & 30.6 & 4.3 & 20.43 & - & 75 \\
\hline $\begin{array}{l}\text { Mamtani et al. } \\
(2020)\end{array}$ & Mamtani & $\begin{array}{l}2020-09- \\
02\end{array}$ & USA & 403 & Hospital & $\begin{array}{l}55^{\wedge} \\
(41-68)\end{array}$ & 32.3 & 9.7 & 12.66 & - & 68 \\
\hline Ren et al. (2020) & Ren & $\begin{array}{l}2020-09- \\
02\end{array}$ & China & 432 & Hospital & NA & 57.9 & 10.0 & - & - & 90 \\
\hline $\begin{array}{l}\text { Mutambudzi et al. } \\
(2020)\end{array}$ & Mutambudzi & $\begin{array}{l}2020-09- \\
03\end{array}$ & UK & 120,075 & $\begin{array}{l}\text { Community } \\
\text { and } \\
\text { Hospital }\end{array}$ & NA & 54.2 & 11.7 & 26.39 & - & 61 \\
\hline Yan et al. (2020) & Yan & $\begin{array}{l}2020-09- \\
07\end{array}$ & China & 578 & Hospital & $\begin{array}{l}49.2^{\wedge} \\
(35-63)\end{array}$ & 49.3 & 9.2 & - & - & - \\
\hline $\begin{array}{l}\text { Mancilla-Galindo } \\
\text { et al. (2020) }\end{array}$ & Mancilla-Galindo & $\begin{array}{l}2020-09- \\
08\end{array}$ & Mexico & 183,779 & $\begin{array}{l}\text { Community } \\
\text { and } \\
\text { Hospital }\end{array}$ & $\begin{array}{l}45^{\wedge} \\
(28-61)\end{array}$ & 46.0 & 7.6 & - & - & - \\
\hline Ullah et al. (2020) & Ullah & $\begin{array}{l}2020-09- \\
08\end{array}$ & UK & 212 & $\begin{array}{l}\text { Community } \\
\text { and } \\
\text { Hospital }\end{array}$ & $\begin{array}{l}66.7 \\
(54.2- \\
80.5)\end{array}$ & 44.8 & 11.3 & 48.11 & - & 37 \\
\hline
\end{tabular}




\begin{tabular}{|c|c|c|c|c|c|c|c|c|c|c|}
\hline Dashti et al. (2020) & Dashti & $\begin{array}{l}2020-09- \\
13\end{array}$ & USA & 12,347 & $\begin{array}{l}\text { and } \\
\text { Hospital }\end{array}$ & $\begin{array}{l}47(32- \\
62)\end{array}$ & 53.3 & 4.6 & 15.87 & - \\
\hline Sami et al. (2020) & Sami & $\begin{array}{l}2020-09- \\
14\end{array}$ & Iran & 490 & $\begin{array}{l}\text { Community } \\
\text { and } \\
\text { Hospital }\end{array}$ & $\begin{array}{l}56.6^{\wedge} \\
(41-71)\end{array}$ & 39.0 & 14.1 & - & - \\
\hline $\begin{array}{l}\text { Pongpirul et al. } \\
(2020)\end{array}$ & Pongpirul & $\begin{array}{l}2020-09- \\
16\end{array}$ & Thailand & 193 & $\begin{array}{l}\text { Community } \\
\text { and } \\
\text { Hospital }\end{array}$ & $\begin{array}{l}37(29- \\
53)\end{array}$ & 41.5 & - & - & 15.03 \\
\hline Burrell et al. (2020) & Burrell & $\begin{array}{l}2020-09- \\
16\end{array}$ & Australia & 204 & Hospital & $\begin{array}{l}63.5 \\
(53-72)\end{array}$ & 31.4 & - & - & 13.24 \\
\hline $\begin{array}{l}\text { Nicholson et al. } \\
(2020)\end{array}$ & Nicholson & $\begin{array}{l}2020-09- \\
17\end{array}$ & USA & 1,042 & Hospital & $\begin{array}{l}64(53- \\
75)\end{array}$ & 43.2 & 8.3 & 22.17 & - \\
\hline Ariza et al. (2020) & Ariza & $\begin{array}{l}2020-09- \\
18\end{array}$ & Colombia & 351 & $\begin{array}{l}\text { Community } \\
\text { and } \\
\text { Hospital }\end{array}$ & $\begin{array}{l}30.5 \\
(\mathrm{NA})\end{array}$ & 54.0 & 6.8 & - & - \\
\hline Carrat et al. (2020) & Carrat & $\begin{array}{l}2020-09- \\
18\end{array}$ & France & 14,628 & Community & NA & 60.3 & 12.0 & 40.83 & - \\
\hline $\begin{array}{l}\text { Favara et al. } \\
(2020)\end{array}$ & Favara & $\begin{array}{l}2020-09- \\
20\end{array}$ & UK & 434 & Community & $\begin{array}{l}40(19- \\
66)\end{array}$ & 82.0 & 8.5 & - & - \\
\hline $\begin{array}{l}\text { Invernizzi et al. } \\
(2020)\end{array}$ & Invernizzi & $\begin{array}{l}2020-09- \\
20\end{array}$ & Italy & 54 & Hospital & $\begin{array}{l}49.9^{\wedge} \\
(34-65)\end{array}$ & 29.7 & - & - & 24.07 \\
\hline Zhu et al. (2020) & Zhu & $\begin{array}{l}2020-09- \\
21\end{array}$ & China & 432 & $\begin{array}{l}\text { Community } \\
\text { and } \\
\text { Hospital }\end{array}$ & $\begin{array}{l}49(35- \\
60)\end{array}$ & 47.9 & 14.4 & - & - \\
\hline $\begin{array}{l}\text { O'Reilly et al. } \\
(2020)\end{array}$ & O'Reilly & $\begin{array}{l}2020-09- \\
21\end{array}$ & Australia & 1,334 & Hospital & NA & - & - & - & 28.49 \\
\hline Meini et al. (n.d.) & Meini & $\begin{array}{l}2020-09- \\
23\end{array}$ & Italy & 461 & Hospital & NA & 51.2 & 10.4 & 25.81 & - \\
\hline $\begin{array}{l}\text { Silva Neto et al. } \\
(2020)\end{array}$ & da Silva Neto & $\begin{array}{l}2020-09- \\
23\end{array}$ & Brazil & 91 & $\begin{array}{l}\text { Community } \\
\text { and } \\
\text { Hospital }\end{array}$ & $\begin{array}{l}49^{\wedge} \\
(29-68)\end{array}$ & 49.4 & - & - & 19.78 \\
\hline $\begin{array}{l}\text { loannou et al. } \\
(2020)\end{array}$ & loannou & $\begin{array}{l}2020-09- \\
23\end{array}$ & USA & 88,747 & $\begin{array}{l}\text { Community } \\
\text { and } \\
\text { Hospital }\end{array}$ & NA & 9.0 & 20.6 & 37.55 & - \\
\hline $\begin{array}{l}\text { Torres-Macho et al. } \\
\text { (2020) }\end{array}$ & Torres-Macho & $\begin{array}{l}2020-09- \\
23\end{array}$ & Spain & 1,968 & Hospital & NA & 44.0 & - & - & 23.37 \\
\hline Li et al. (2020) & Li, Cai & $\begin{array}{l}2020-09- \\
28\end{array}$ & China & 98 & Hospital & $\begin{array}{l}68.5 \\
(63-75)\end{array}$ & 58.2 & - & - & 11.22 \\
\hline Wang et al. (2020) & Wang & $\begin{array}{l}2020-09- \\
29\end{array}$ & USA & 1,078 & Hospital & NA & 38.2 & 3.7 & 24.86 & - \\
\hline $\begin{array}{l}\text { Lopez-Medrano et } \\
\text { al. (2020) }\end{array}$ & Lopez-Medrano & $\begin{array}{l}2020-09- \\
30\end{array}$ & Spain & 261 & Hospital & NA & 43.7 & - & - & 37.16 \\
\hline $\begin{array}{l}\text { Collard et al. } \\
(2020)\end{array}$ & Collard & $\begin{array}{l}2020-10- \\
01\end{array}$ & Netherlands & 1,604 & Hospital & $\begin{array}{l}65.7^{\wedge} \\
(50-80)\end{array}$ & 39.5 & 4.9 & - & - \\
\hline $\begin{array}{l}\text { Makaronidis et al. } \\
(2020)\end{array}$ & Makaronidis & $\begin{array}{l}2020-10- \\
01\end{array}$ & UK & 567 & Community & $\begin{array}{l}39.4^{\wedge} \\
(27-51)\end{array}$ & 69.1 & 9.3 & - & - \\
\hline $\begin{array}{l}\text { Yadaw et al. } \\
(2020)\end{array}$ & Yadaw & $\begin{array}{l}2020-10- \\
01\end{array}$ & USA & 5,051 & $\begin{array}{l}\text { Community } \\
\text { and } \\
\text { Hospital }\end{array}$ & NA & - & 3.6 & 15.92 & - \\
\hline \multirow[t]{2}{*}{$\begin{array}{l}\text { Talavera et al. } \\
(2020)\end{array}$} & Talavera & $\begin{array}{l}2020-10- \\
01\end{array}$ & Spain & 576 & Hospital & $\begin{array}{l}67.2^{\wedge} \\
(52-81)\end{array}$ & 43.4 & - & - & 20.49 \\
\hline & & 2020-10- & & $\ldots$ & Community & & 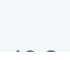 & $\cdots$ & $\ldots$ & \\
\hline
\end{tabular}




\begin{tabular}{|c|c|c|c|c|c|c|c|c|c|c|c|}
\hline Jakob et al. (2020) & Jakob & 01 & Multiple & 2,155 & $\begin{array}{l}\text { and } \\
\text { Hospital }\end{array}$ & NA & 40.3 & 6.6 & 7.29 & - & 34. \\
\hline Incerti et al. (2020) & Incerti & $\begin{array}{l}2020-10- \\
02\end{array}$ & USA & 13,658 & Hospital & $\begin{array}{l}62(49- \\
75)\end{array}$ & 48.1 & 6.3 & 22.64 & - & 45. \\
\hline Luo et al. (2020) & Luo, Rizvi & $\begin{array}{l}2020-10- \\
03\end{array}$ & USA & 102 & Hospital & $\begin{array}{l}68(61- \\
75)\end{array}$ & 52.0 & - & - & 26.47 & - \\
\hline $\begin{array}{l}\text { Robinson et al. } \\
\text { (2021) }\end{array}$ & Robinson & $\begin{array}{l}2020-10- \\
05\end{array}$ & USA & 3,248 & Hospital & $\begin{array}{l}51^{\wedge} \\
(34-68)\end{array}$ & 72.0 & 4.0 & 17.64 & - & 61. \\
\hline Adrish et al. (2020) & Adrish & $\begin{array}{l}2020-10- \\
05\end{array}$ & USA & 1,173 & Hospital & NA & 38.6 & 14.0 & 14.66 & - & 71. \\
\hline $\begin{array}{l}\text { Chaudhary et al. } \\
(2020)\end{array}$ & Chaudhary & $\begin{array}{l}2020-10- \\
06\end{array}$ & Nepal & 220 & Hospital & $\begin{array}{l}31.5 \\
(25-37)\end{array}$ & 17.7 & 11.4 & 7.73 & - & 80. \\
\hline $\begin{array}{l}\text { Raines et al. } \\
(2021)\end{array}$ & Raines & $\begin{array}{l}2020-10- \\
07\end{array}$ & USA & 453 & $\begin{array}{l}\text { Community } \\
\text { and } \\
\text { Hospital }\end{array}$ & $\begin{array}{l}60.8^{\wedge} \\
(46-74)\end{array}$ & 10.7 & - & - & 52.98 & 41. \\
\hline $\begin{array}{l}\text { Roederer et al. } \\
\text { (2021) }\end{array}$ & Roederer & $\begin{array}{l}2020-10- \\
09\end{array}$ & France & 818 & Community & NA & 20.4 & 36.9 & 8.80 & - & 53. \\
\hline Zinellu et al. (2021) & Zinellu & $\begin{array}{l}2020-10- \\
11\end{array}$ & Italy & 105 & Hospital & $\begin{array}{l}72 \\
(59.5- \\
80)\end{array}$ & 33.3 & 30.5 & 10.48 & - & 59. \\
\hline $\begin{array}{l}\text { Ramachandran et } \\
\text { al. (2020) }\end{array}$ & Ramachandran & $\begin{array}{l}2020-10- \\
12\end{array}$ & USA & 188 & Hospital & NA & - & 18.6 & - & - & - \\
\hline $\begin{array}{l}\text { Lamure et al. } \\
(2020)\end{array}$ & Lamure & $\begin{array}{l}2020-10- \\
12\end{array}$ & France & 89 & Hospital & $\begin{array}{l}67(19- \\
92)\end{array}$ & 34.0 & 5.6 & 32.58 & - & 48. \\
\hline Ghinai et al. (2020) & Ghinai & $\begin{array}{l}2020-10- \\
12\end{array}$ & USA & 1,435 & $\begin{array}{l}\text { Homeless } \\
\text { Shelters }\end{array}$ & NA & 27.6 & 36.6 & 17.49 & - & 33. \\
\hline Best et al. (2020) & Best & $\begin{array}{l}2020-10- \\
12\end{array}$ & USA & 3,471 & Hospital & $\begin{array}{l}63.5^{\wedge} \\
(47-79)\end{array}$ & 51.2 & - & - & 28.64 & - \\
\hline $\begin{array}{l}\text { Savarraj et al. } \\
(2020)\end{array}$ & Savarraj & $\begin{array}{l}2020-10- \\
18\end{array}$ & USA & 48 & Hospital & $\begin{array}{l}50^{\wedge} \\
(33-67)\end{array}$ & 48.0 & 10.4 & - & - & - \\
\hline Israel et al. (2020) & Israel, Schaffer & $\begin{array}{l}2020-10- \\
18\end{array}$ & Israel & 26,959 & Hospital & NA & 50.6 & 6.8 & 15.22 & - & 77. \\
\hline $\begin{array}{l}\text { El-Solh et al. } \\
(2020)\end{array}$ & El-Solh & $\begin{array}{l}2020-10- \\
20\end{array}$ & USA & 7,816 & Hospital & $\begin{array}{l}69(60- \\
74)\end{array}$ & 5.5 & - & - & 45.25 & - \\
\hline Perico et al. (2020) & Perico & $\begin{array}{l}2020-10- \\
22\end{array}$ & Italy & 423 & Community & $\begin{array}{l}44.3^{\wedge} \\
(34-54)\end{array}$ & 36.4 & 21.7 & 17.97 & - & 60. \\
\hline Zhou et al. (2020) & Zhou, Song & $\begin{array}{l}2020-10- \\
22\end{array}$ & China & 124 & Hospital & $\begin{array}{l}67(30- \\
86)\end{array}$ & 48.0 & 19.1 & - & - & - \\
\hline $\begin{array}{l}\text { Chudasama et al. } \\
(2020)\end{array}$ & Chudasama & $\begin{array}{l}2020-10- \\
23\end{array}$ & UK & 1,706 & $\begin{array}{l}\text { Community } \\
\text { and } \\
\text { Hospital }\end{array}$ & $\begin{array}{l}68(48- \\
85)\end{array}$ & 42.5 & 13.8 & 40.97 & - & 45. \\
\hline $\begin{array}{l}\text { Salama et al. } \\
(2021)\end{array}$ & Salama & $\begin{array}{l}2020-10- \\
23\end{array}$ & Multiple & 377 & Hospital & $\begin{array}{l}55.9^{\wedge} \\
(41-70)\end{array}$ & 40.8 & 5.8 & 16.98 & - & 77. \\
\hline Wang et al. (2020) & Wang, Zheutlin & $\begin{array}{l}2020-10- \\
26\end{array}$ & USA & 3,273 & Hospital & $\begin{array}{l}65 \text { (53- } \\
77)\end{array}$ & 42.7 & 3.5 & 20.68 & - & 53. \\
\hline
\end{tabular}




\begin{tabular}{|c|c|c|c|c|c|c|c|c|c|c|c|}
\hline$(2020)$ & 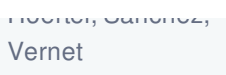 & $\begin{array}{l}\text {-uー } \\
27\end{array}$ & France & 12,210 & Hospital & NA & 50.1 & 9.0 & - & - & - \\
\hline Arleo et al. (2020) & Arleo & $\begin{array}{l}2020-10- \\
27\end{array}$ & USA & 70 & $\begin{array}{l}\text { Community } \\
\text { and } \\
\text { Hospital }\end{array}$ & $\begin{array}{l}56.6^{\wedge} \\
(48-65)\end{array}$ & 80.0 & 1.4 & 28.57 & - & 70. \\
\hline $\begin{array}{l}\text { Bermejo-Martin et } \\
\text { al. (2020) }\end{array}$ & Bermejo-Martin & $\begin{array}{l}2020-10- \\
27\end{array}$ & Spain & 250 & $\begin{array}{l}\text { Community } \\
\text { and } \\
\text { Hospital }\end{array}$ & NA & 64.0 & 6.0 & - & - & - \\
\hline $\begin{array}{l}\text { Joubert et al. } \\
(2020)\end{array}$ & Joubert & $\begin{array}{l}2020-10- \\
29\end{array}$ & France & 74 & $\begin{array}{l}\text { Community } \\
\text { and } \\
\text { Hospital }\end{array}$ & NA & - & 5.4 & - & - & - \\
\hline $\begin{array}{l}\text { Kortela et al. } \\
(2020)\end{array}$ & Kortela & $\begin{array}{l}2020-11- \\
01\end{array}$ & Finland & 3,008 & $\begin{array}{l}\text { Community } \\
\text { and } \\
\text { Hospital }\end{array}$ & $\begin{array}{l}51(36- \\
69)\end{array}$ & 59.6 & 11.0 & 14.03 & - & 29. \\
\hline $\begin{array}{l}\text { Gianfrancesco et } \\
\text { al. }(2020)\end{array}$ & $\begin{array}{l}\text { Gianfrancesco, } \\
\text { Leykina }\end{array}$ & $\begin{array}{l}2020-11- \\
03\end{array}$ & USA & 1,324 & $\begin{array}{l}\text { Community } \\
\text { and } \\
\text { Hospital }\end{array}$ & NA & 75.9 & - & - & 26.66 & 68. \\
\hline $\begin{array}{l}\text { Gallichotte et al. } \\
(2020)\end{array}$ & Gallichotte & $\begin{array}{l}2020-11- \\
05\end{array}$ & USA & 239 & Community & $\begin{array}{l}41^{\wedge} \\
(16-76)\end{array}$ & - & 20.1 & 16.74 & - & 57. \\
\hline Lin et al. (2020) & Lin & $\begin{array}{l}2020-11- \\
05\end{array}$ & USA & 2,821 & Hospital & $\begin{array}{l}62.7^{\wedge} \\
(\mathrm{NA})\end{array}$ & 45.0 & 2.9 & 12.51 & - & 3.3 \\
\hline Kim et al. (2020) & Kim, Han & $\begin{array}{l}2020-11- \\
09\end{array}$ & $\begin{array}{l}\text { South } \\
\text { Korea }\end{array}$ & 4,787 & Hospital & $\begin{array}{l}55(38- \\
68)\end{array}$ & 60.1 & 5.5 & 2.84 & - & 91. \\
\hline Galal et al. (2020) & Galal & $\begin{array}{l}2020-11- \\
12\end{array}$ & Egypt & 430 & Community & $\begin{array}{l}37.4^{\wedge} \\
(24-50)\end{array}$ & 63.7 & 6.0 & 7.67 & - & 86. \\
\hline Sourij et al. (2020) & Sourij & $\begin{array}{l}2020-11- \\
16\end{array}$ & Austria & 238 & Hospital & $\begin{array}{l}71.1^{\wedge} \\
(58-83)\end{array}$ & 36.1 & 1.7 & 15.97 & - & 82. \\
\hline $\begin{array}{l}\text { Clavario et al. } \\
(2020)\end{array}$ & Clavario & $\begin{array}{l}2020-11- \\
16\end{array}$ & Italy & 110 & Community & $\begin{array}{l}61.7 \\
(53.5- \\
69.2)\end{array}$ & 40.9 & 40.9 & - & - & - \\
\hline Saeed et al. (2020) & Saeed & $\begin{array}{l}2020-11- \\
16\end{array}$ & $\begin{array}{l}\text { United Arab } \\
\text { Emirates }\end{array}$ & 173 & Hospital & NA & 34.1 & 6.4 & - & - & - \\
\hline $\begin{array}{l}\text { Mansour et al. } \\
(2020)\end{array}$ & Mansour & $\begin{array}{l}2020-11- \\
16\end{array}$ & Iran & 353 & Hospital & $\begin{array}{l}61.7^{\wedge} \\
(45-78)\end{array}$ & 42.5 & 7.1 & - & - & - \\
\hline $\begin{array}{l}\text { Cadegiani et al. } \\
(2020)\end{array}$ & Cadegiani & $\begin{array}{l}2020-11- \\
18\end{array}$ & Brazil & 130 & Community & $\begin{array}{l}42^{\wedge} \\
(\mathrm{NA})\end{array}$ & 0.0 & 7.7 & - & - & - \\
\hline Ilic et al. (2021) & Ilic & $\begin{array}{l}2020-11- \\
19\end{array}$ & Serbia & 107 & $\begin{array}{l}\text { Community } \\
\text { and } \\
\text { Hospital }\end{array}$ & $\begin{array}{l}39.1^{\wedge} \\
(27-50)\end{array}$ & - & 29.9 & - & - & - \\
\hline $\begin{array}{l}\text { Benaim et al. } \\
(2020)\end{array}$ & Benaim & $\begin{array}{l}2020-11- \\
19\end{array}$ & Israel & 693 & Hospital & $\begin{array}{l}59.8 \\
(\mathrm{NA})\end{array}$ & 47.9 & - & - & 5.05 & - \\
\hline Ho et al. (2020) & Ho & $\begin{array}{l}2020-11- \\
19\end{array}$ & UK & 235,928 & $\begin{array}{l}\text { Community } \\
\text { and } \\
\text { Hospital }\end{array}$ & NA & - & - & - & 45.38 & - \\
\hline Singh et al. (2020) & Singh & $\begin{array}{l}2020-11- \\
20\end{array}$ & UK & 930 & Hospital & $\begin{array}{l}71.4^{\wedge} \\
(54-87)\end{array}$ & 44.8 & - & - & 19.03 & - \\
\hline $\begin{array}{l}\text { Márquez-Salinas } \\
\text { et al. (2020) }\end{array}$ & Marquez-Salinas & $\begin{array}{l}2020-11- \\
24\end{array}$ & Mexico & 1,068 & Hospital & $\begin{array}{l}53(44- \\
63)\end{array}$ & 36.8 & - & - & 14.98 & - \\
\hline $\begin{array}{l}\text { Díez-Manglano et } \\
\text { al. (2020) }\end{array}$ & Diez-Manglanas & $\begin{array}{l}2020-11- \\
24\end{array}$ & Spain & 4,393 & Hospital & $\begin{array}{l}53^{\wedge} \\
(\mathrm{NA})\end{array}$ & 40.8 & 6.6 & - & - & - \\
\hline Bellan et al. (2020) & Bellan & $\begin{array}{l}2020-11- \\
26\end{array}$ & Italy & 1,697 & Hospital & $\begin{array}{l}71(58- \\
80)\end{array}$ & 41.0 & 3.2 & - & - & - \\
\hline
\end{tabular}




\begin{tabular}{|c|c|c|c|c|c|c|c|c|c|c|}
\hline $\begin{array}{l}\text { Castilla-Bancayán } \\
(2020)\end{array}$ & Woolcott & 26 & Mexico & $1,636,050$ & $\begin{array}{l}\text { and } \\
\text { Hospital }\end{array}$ & $54)$ & 51.9 & - & - & 8.76 \\
\hline Yao et al. (2021) & Yao, Hasegawa & $\begin{array}{l}2020-11- \\
26\end{array}$ & Japan & 101 & Hospital & $\begin{array}{l}60^{\wedge} \\
(17-97)\end{array}$ & 39.6 & - & - & 28.71 \\
\hline Chen et al. (2020) & Chen, Varathraja & $\begin{array}{l}2020-11- \\
29\end{array}$ & USA & 10,123 & $\begin{array}{l}\text { Community } \\
\text { and } \\
\text { Hospital }\end{array}$ & $\begin{array}{l}40(28- \\
54)\end{array}$ & 53.6 & 4.4 & 9.71 & - \\
\hline $\begin{array}{l}\text { Serling-Boyd et al. } \\
\text { (2020) }\end{array}$ & Serling-Boyd & $\begin{array}{l}2020-11- \\
30\end{array}$ & USA & 831 & Hospital & NA & 76.0 & 3.0 & 23.23 & - \\
\hline $\begin{array}{l}\text { Simons et al. } \\
(2020)\end{array}$ & Simons & $\begin{array}{l}2020-11- \\
30\end{array}$ & UK & 446 & Hospital & $\begin{array}{l}64.9 \\
(52.4- \\
76.2)\end{array}$ & 35.9 & 9.4 & 38.57 & - \\
\hline $\begin{array}{l}\text { Dupraz et al. } \\
(2020)\end{array}$ & Dupraz & $\begin{array}{l}2020-11- \\
30\end{array}$ & Switzerland & 219 & Community & NA & 54.8 & 11.0 & - & - \\
\hline $\begin{array}{l}\text { Barasa et al. } \\
(2020)\end{array}$ & Barasa & $\begin{array}{l}2020-11- \\
30\end{array}$ & USA & 394 & Hospital & NA & 47.7 & 14.7 & 36.04 & - \\
\hline Ren et al. (2020) & Ren, Guo & $\begin{array}{l}2020-11- \\
30\end{array}$ & China & 481 & Hospital & NA & 45.7 & 7.7 & 0.62 & - \\
\hline $\begin{array}{l}\text { Martinez- } \\
\text { Lacalzada et al. } \\
(2020)\end{array}$ & $\begin{array}{l}\text { Martinez- } \\
\text { Lacalzada }\end{array}$ & $\begin{array}{l}2020-12- \\
01\end{array}$ & Spain & 10,433 & Hospital & $\begin{array}{l}65.8^{\wedge} \\
(49-82)\end{array}$ & 42.8 & 5.2 & 23.77 & - \\
\hline Li et al. (2020) & Li, Long, Zhang & $\begin{array}{l}2020-12- \\
03\end{array}$ & China & 954 & Hospital & NA & - & - & - & 5.87 \\
\hline Martini et al. (2020) & Martini & $\begin{array}{l}2020-12- \\
04\end{array}$ & Italy & 146 & Hospital & NA & 49.0 & - & - & 46.58 \\
\hline $\begin{array}{l}\text { O'Gallagher et al. } \\
(2020)\end{array}$ & O'Gallagher & $\begin{array}{l}2020-12- \\
04\end{array}$ & UK & 1,721 & Hospital & $\begin{array}{l}71(56- \\
83)\end{array}$ & 43.4 & 6.6 & 18.48 & - \\
\hline $\begin{array}{l}\text { Alguwaihes et al. } \\
(2020)\end{array}$ & Alguwaihes & $\begin{array}{l}2020-12- \\
05\end{array}$ & $\begin{array}{l}\text { Saudi } \\
\text { Arabia }\end{array}$ & 439 & Hospital & $\begin{array}{l}55(19- \\
101)\end{array}$ & 31.7 & 2.1 & - & - \\
\hline Zuo et al. (2020) & Zuo, Warnock & $\begin{array}{l}2020-12- \\
05\end{array}$ & USA & 118 & Hospital & $\begin{array}{l}61^{\wedge} \\
(44-78)\end{array}$ & 46.0 & - & - & 23.73 \\
\hline Zhang et al. (2020) & Zhang, Li & $\begin{array}{l}2020-12- \\
06\end{array}$ & UK & 1,746 & $\begin{array}{l}\text { Community } \\
\text { and } \\
\text { Hospital }\end{array}$ & $\begin{array}{l}68.8^{\wedge} \\
(59-78)\end{array}$ & 47.1 & 10.1 & 35.09 & - \\
\hline Dai et al. (2020) & Dai & $\begin{array}{l}2020-12- \\
09\end{array}$ & China & 1,574 & Hospital & $\begin{array}{l}57.3^{\wedge} \\
(41-73)\end{array}$ & 48.2 & - & - & 9.21 \\
\hline $\begin{array}{l}\text { Vila-Córcoles et al. } \\
\text { (2020) }\end{array}$ & Vila-Corcoles & $\begin{array}{l}2020-12- \\
10\end{array}$ & Spain & 79,083 & Community & NA & 52.4 & - & - & 16.12 \\
\hline Bisso et al. (2020) & Bisso & $\begin{array}{l}2020-12- \\
11\end{array}$ & Argentina & 168 & Hospital & $\begin{array}{l}67(58- \\
75)\end{array}$ & 34.0 & 10.7 & - & - \\
\hline $\begin{array}{l}\text { Rentsch et al. } \\
(2020)\end{array}$ & Rentsch, Beckman & $\begin{array}{l}2020-12- \\
11\end{array}$ & USA & 4,297 & Hospital & $\begin{array}{l}68(58- \\
75)\end{array}$ & 6.6 & 36.8 & 39.31 & - \\
\hline $\begin{array}{l}\text { Thiabaud et al. } \\
\text { (2020) }\end{array}$ & Thiabaud & $\begin{array}{l}2020-12- \\
11\end{array}$ & Switzerland & 3,582 & Hospital & $\begin{array}{l}68(54- \\
79)\end{array}$ & 40.5 & - & - & 6.62 \\
\hline $\begin{array}{l}\text { Vila-Corcoles et al. } \\
(2020)\end{array}$ & $\begin{array}{l}\text { Vila-Corcoles, } \\
\text { Satue-Gracia }\end{array}$ & $\begin{array}{l}2020-12- \\
11\end{array}$ & Spain & 282 & $\begin{array}{l}\text { Community } \\
\text { and } \\
\text { Hospital }\end{array}$ & $\begin{array}{l}65.9^{\wedge} \\
(53-78)\end{array}$ & 50.3 & 8.9 & - & - \\
\hline Lévy et al. (2020) & Levy & $\begin{array}{l}2020-12- \\
12\end{array}$ & France & 61 & Hospital & $\begin{array}{l}60(50- \\
69)\end{array}$ & 20.0 & 8.2 & - & - \\
\hline $\begin{array}{l}\text { Kantele et al. } \\
(2020)\end{array}$ & Kantele & $\begin{array}{l}2020-12- \\
13\end{array}$ & Finland & 1,095 & $\begin{array}{l}\text { Community } \\
\text { and } \\
\text { Hospital }\end{array}$ & $\begin{array}{l}38(31- \\
48)\end{array}$ & 82.7 & 16.3 & 25.84 & - \\
\hline
\end{tabular}




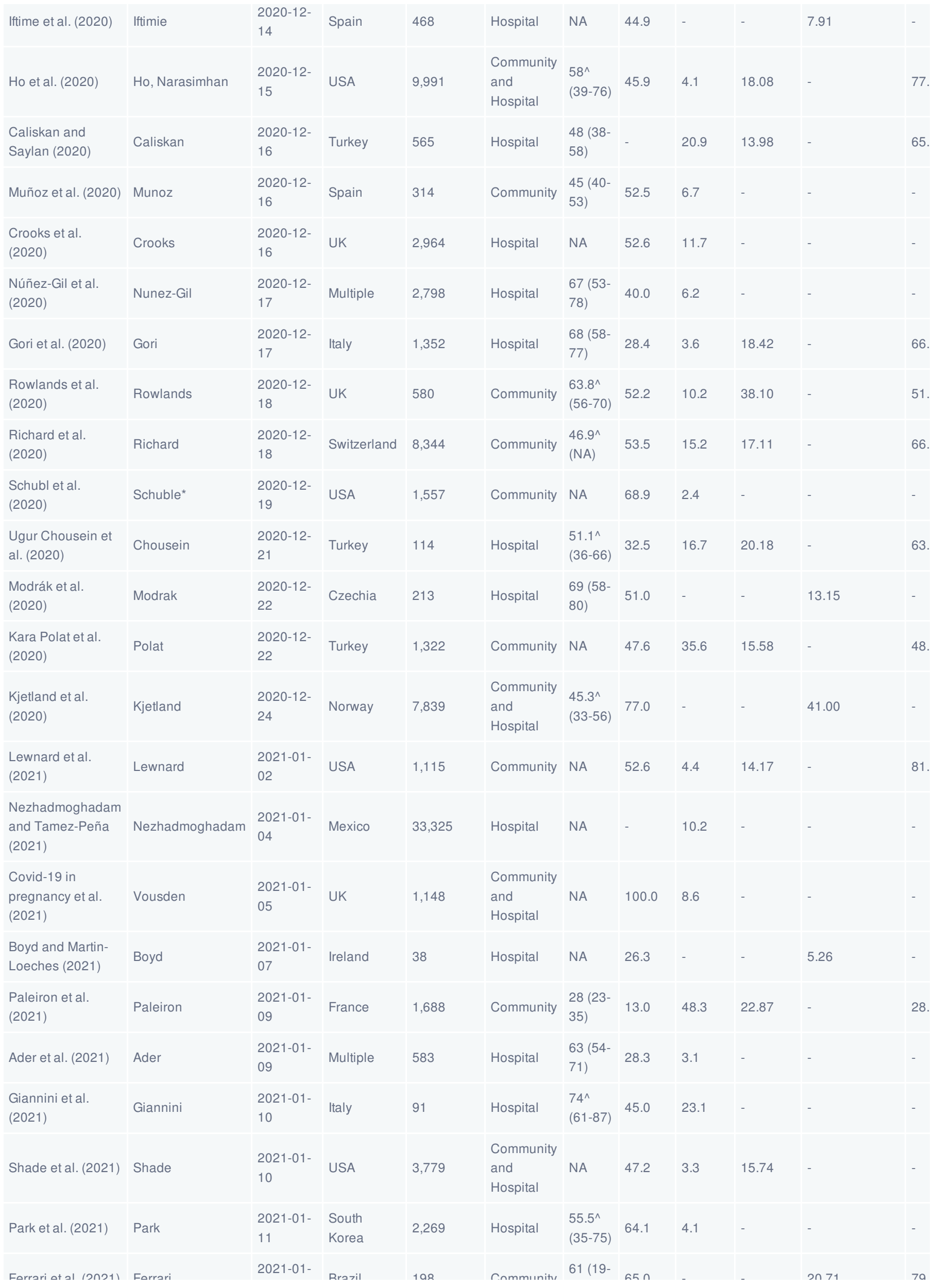




\begin{tabular}{|c|c|c|c|c|c|c|c|c|c|c|c|}
\hline ' viluirvial. (cva') & 'vinil & 12 & Licu<" & ivo & 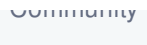 & 91) & uง.v & & & cu.t. & ive \\
\hline Zhang et al. (2021) & Zhang, Yang & $\begin{array}{l}2021-01- \\
12\end{array}$ & UK & 1,485 & $\begin{array}{l}\text { Community } \\
\text { and } \\
\text { Hospital }\end{array}$ & $\begin{array}{l}68.2^{\wedge} \\
(59-77)\end{array}$ & 47.2 & 48.5 & 39.19 & - & 11. \\
\hline $\begin{array}{l}\text { Ebrahimian et al. } \\
\text { (2021) }\end{array}$ & Ebrahimian & $\begin{array}{l}2021-01- \\
13\end{array}$ & USA & 226 & Hospital & NA & - & - & - & 10.62 & - \\
\hline $\begin{array}{l}\text { Vahidy et al. } \\
(2021)\end{array}$ & Vahidy & $\begin{array}{l}2021-01- \\
13\end{array}$ & USA & 96,473 & Hospital & $\begin{array}{l}51.2^{\wedge} \\
(32-69)\end{array}$ & 59.6 & - & - & 26.18 & 73. \\
\hline $\begin{array}{l}\text { Mendes et al. } \\
(2021)\end{array}$ & Mendes & $\begin{array}{l}2021-01- \\
14\end{array}$ & Switzerland & 265 & Hospital & $\begin{array}{l}85.9^{\wedge} \\
(79-92)\end{array}$ & 57.0 & 5.7 & - & - & - \\
\hline $\begin{array}{l}\text { Saurabh et al. } \\
(2021)\end{array}$ & Saurabh & $\begin{array}{l}2021-01- \\
14\end{array}$ & India & 911 & Community & $\begin{array}{l}43.1^{\wedge} \\
(23-62)\end{array}$ & 35.2 & 8.1 & 0.66 & - & 91. \\
\hline $\begin{array}{l}\text { Thakur et al. } \\
\text { (2021) }\end{array}$ & Thakar & $\begin{array}{l}2021-01- \\
18\end{array}$ & India & 250 & Hospital & NA & 42.4 & - & - & 49.20 & - \\
\hline Zhong et al. (2021) & Zhong & $\begin{array}{l}2021-01- \\
18\end{array}$ & China & 91 & Hospital & $\begin{array}{l}47.3^{\wedge} \\
(30-64)\end{array}$ & 49.5 & - & - & 18.68 & - \\
\hline $\begin{array}{l}\text { Cummins et al. } \\
\text { (2021) }\end{array}$ & Cummins & $\begin{array}{l}2021-01- \\
20\end{array}$ & UK & 1,781 & $\begin{array}{l}\text { Community } \\
\text { and } \\
\text { Hospital }\end{array}$ & NA & 44.8 & 10.2 & - & - & - \\
\hline Sun et al. (2021) & Sun & $\begin{array}{l}2021-01- \\
21\end{array}$ & USA & 323 & $\begin{array}{l}\text { Community } \\
\text { and } \\
\text { Hospital }\end{array}$ & NA & 57.6 & - & - & 39.32 & - \\
\hline $\begin{array}{l}\text { Caglar and Kacer } \\
(2021)\end{array}$ & Caglar & $\begin{array}{l}2021-01- \\
25\end{array}$ & Turkey & 120 & Hospital & $\begin{array}{l}57(47- \\
67)\end{array}$ & 51.7 & - & - & 37.50 & - \\
\hline Quan et al. (2021) & Quan & $\begin{array}{l}2021-01- \\
27\end{array}$ & USA & 2,038 & Hospital & $\begin{array}{l}64^{\wedge} \\
(47-80)\end{array}$ & 50.4 & - & - & 39.74 & - \\
\hline $\begin{array}{l}\text { Strangfeld et al. } \\
\text { (2021) }\end{array}$ & Strangfeld & $\begin{array}{l}2021-01- \\
27\end{array}$ & Multiple & 3,729 & $\begin{array}{l}\text { Community } \\
\text { and } \\
\text { Hospital }\end{array}$ & $\begin{array}{l}57^{\wedge} \\
(41-72)\end{array}$ & 68.0 & - & - & 20.81 & 64. \\
\hline Tardif et al. (2021) & Tardif & $\begin{array}{l}2021-01- \\
27\end{array}$ & Canada & 4,488 & $\begin{array}{l}\text { Community } \\
\text { and } \\
\text { Hospital }\end{array}$ & NA & 53.9 & 9.6 & 33.60 & - & 56. \\
\hline Jafari et al. (2021) & Jafari & $\begin{array}{l}2021-01- \\
28\end{array}$ & USA & 1,979 & Hospital & $\begin{array}{l}66(57- \\
74)\end{array}$ & 32.0 & 12.1 & - & - & - \\
\hline $\begin{array}{l}\text { Prats-Uribe et al. } \\
(2021)\end{array}$ & Prats-Uribe & $\begin{array}{l}2021-01- \\
30\end{array}$ & Spain & 696 & Hospital & $\begin{array}{l}63^{\wedge} \\
(52-73)\end{array}$ & 30.9 & - & - & 16.09 & 74. \\
\hline Nuño et al. (2021) & Nuno & $\begin{array}{l}2021-02- \\
01\end{array}$ & USA & 4,730 & Hospital & $\begin{array}{l}61(46- \\
73)\end{array}$ & 43.6 & 10.1 & - & - & - \\
\hline Abajo et al. (2021) & de Abajo & $\begin{array}{l}2021-02- \\
03\end{array}$ & Spain & 625 & Hospital & NA & 39.4 & 4.6 & 29.28 & - & 39. \\
\hline Mora et al. (2021) & Mora & $\begin{array}{l}2021-02- \\
03\end{array}$ & USA & 1,058 & Community & $\begin{array}{l}39.7^{\wedge} \\
(27-52)\end{array}$ & 52.5 & 4.5 & 14.56 & - & 80. \\
\hline $\begin{array}{l}\text { Molenaar et al. } \\
\text { (2021) }\end{array}$ & Molenaar & $\begin{array}{l}2021-02- \\
03\end{array}$ & USA & 696 & $\begin{array}{l}\text { Community } \\
\text { and } \\
\text { Hospital }\end{array}$ & $\begin{array}{l}33.1^{\wedge} \\
(\mathrm{NA})\end{array}$ & 100.0 & 4.5 & - & - & - \\
\hline
\end{tabular}




\begin{tabular}{|c|c|c|c|c|c|c|c|c|c|c|c|}
\hline $\begin{array}{l}\text { Didikoglu et al. } \\
\text { (2021) }\end{array}$ & Didikoglu & $\begin{array}{l}2021-02- \\
04\end{array}$ & UK & 384,816 & $\begin{array}{l}\text { Community } \\
\text { and } \\
\text { Hospital }\end{array}$ & $\begin{array}{l}68.3^{\wedge} \\
(60-76)\end{array}$ & 54.9 & 9.8 & 35.19 & - & 55. \\
\hline Estiri et al. (2021) & Estiri & $\begin{array}{l}2021-02- \\
04\end{array}$ & USA & 16,709 & Hospital & NA & 57.2 & - & - & 8.62 & - \\
\hline $\begin{array}{l}\text { Tavakol et al. } \\
(2021)\end{array}$ & Tavakol & $\begin{array}{l}2021-02- \\
04\end{array}$ & Iran & 206 & $\begin{array}{l}\text { Community } \\
\text { and } \\
\text { Hospital }\end{array}$ & $\begin{array}{l}40.9^{\wedge} \\
(29-52)\end{array}$ & 57.8 & - & - & 12.62 & - \\
\hline Cai et al. (2021) & Cai, Yang & $\begin{array}{l}2021-02- \\
05\end{array}$ & China & 455 & Hospital & NA & 52.6 & 7.5 & - & - & - \\
\hline Lohia et al. (2021) & Lohia & $\begin{array}{l}2021-02- \\
05\end{array}$ & USA & 1,871 & Hospital & $\begin{array}{l}64.1^{\wedge} \\
(48-80)\end{array}$ & 48.4 & - & - & 37.63 & - \\
\hline $\begin{array}{l}\text { Vila-Corcoles et al. } \\
(2021)\end{array}$ & $\begin{array}{l}\text { Vila-Corcoles, } \\
\text { Satue-Gracia, Vila- } \\
\text { Rovira }\end{array}$ & $\begin{array}{l}2021-02- \\
05\end{array}$ & Spain & 79,083 & Community & $\begin{array}{l}65.8^{\wedge} \\
(54-77)\end{array}$ & 52.4 & 16.1 & - & - & - \\
\hline $\begin{array}{l}\text { International } \\
\text { Severe Acute } \\
\text { Respiratory and } \\
\text { emerging } \\
\text { Infections } \\
\text { Consortium et al. } \\
(2020)\end{array}$ & ISARIC & $\begin{array}{l}2021-02- \\
11\end{array}$ & Multiple & 240,149 & Hospital & $\begin{array}{l}60 \\
\text { (NA) }\end{array}$ & 49.0 & - & - & 3.63 & 21. \\
\hline $\begin{array}{l}\text { Gégout petit et al. } \\
(2021)\end{array}$ & Gegout Petit & $\begin{array}{l}2021-02- \\
12\end{array}$ & France & 2,006 & Community & NA & 55.0 & 16.8 & - & - & - \\
\hline Zhang et al. (2021) & Zhang, Wang & $\begin{array}{l}2021-02- \\
13\end{array}$ & China & 172 & Hospital & $\begin{array}{l}47.9^{\wedge} \\
(29-66)\end{array}$ & 46.5 & 7.0 & 1.16 & 91.86 & - \\
\hline 4 & & & & & & & & & & & 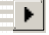 \\
\hline
\end{tabular}

* grouped together current smokers and vapers

\section{Smoking prevalence by country}

Unadjusted smoking prevalence compared with overall estimates for national adult smoking prevalence split by country and study setting is presented in Figure $2 \mathrm{a}$ and $2 \mathrm{~b}$. Lower than expected current smoking prevalence was generally observed, especially in studies with hospitalised samples. Former smoking prevalence was more similar to expected prevalence when reported; however, study-based prevalence was typically higher than national estimates. National smoking prevalence estimates used for comparison are presented in Supplementary table 3. 


\section{Prevalence of current smoking in included studies}

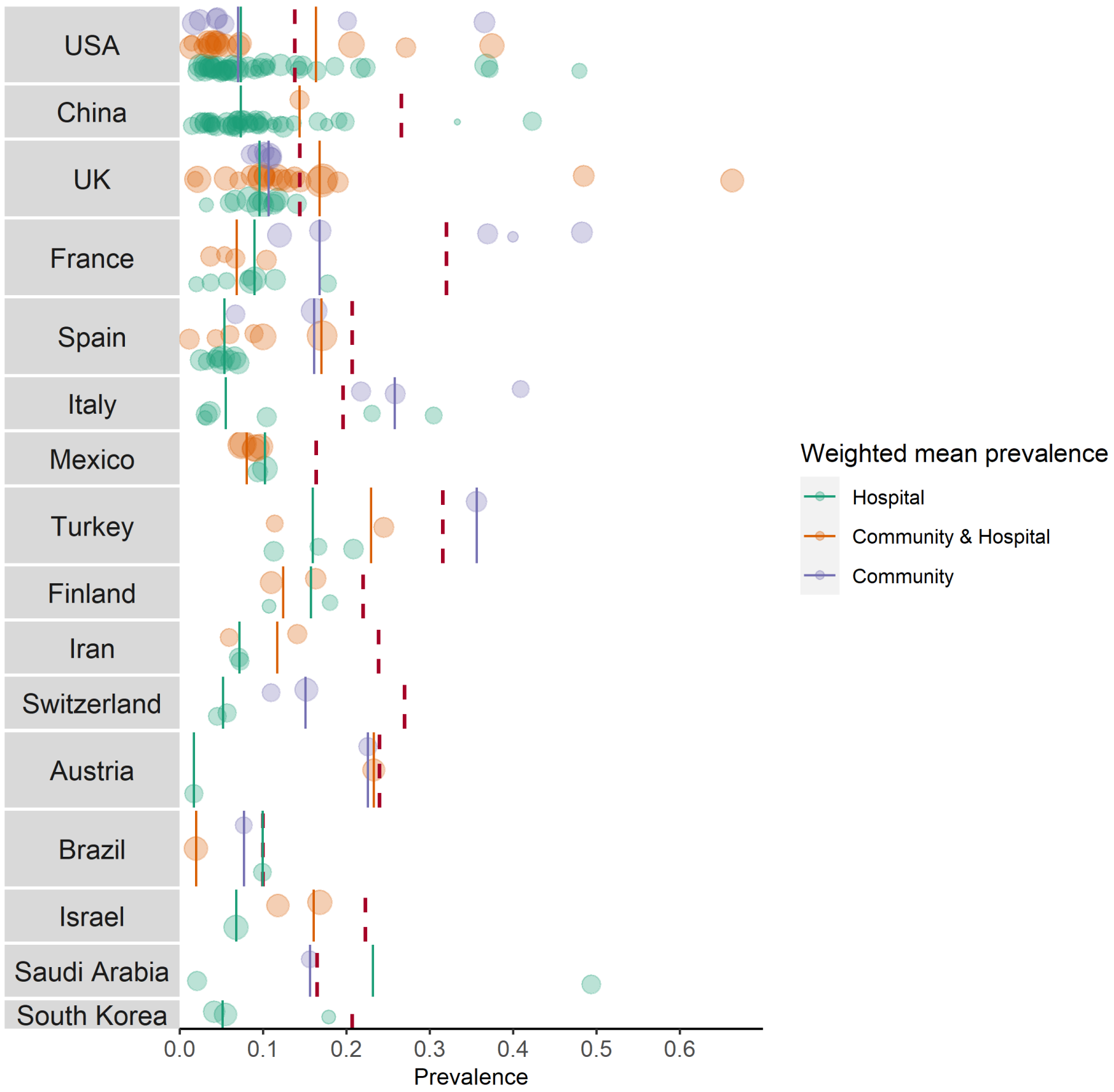

Figure 2a. Weighted mean prevalence of current smoking in included studies, split by country (solid lines). The circles represent individual studies, with colour corresponding to study setting (i.e. community, community and hospital, hospital) and size corresponding to relative study sample size. For comparison, national current smoking prevalence is shown by the dashed red lines. Countries with three or more 


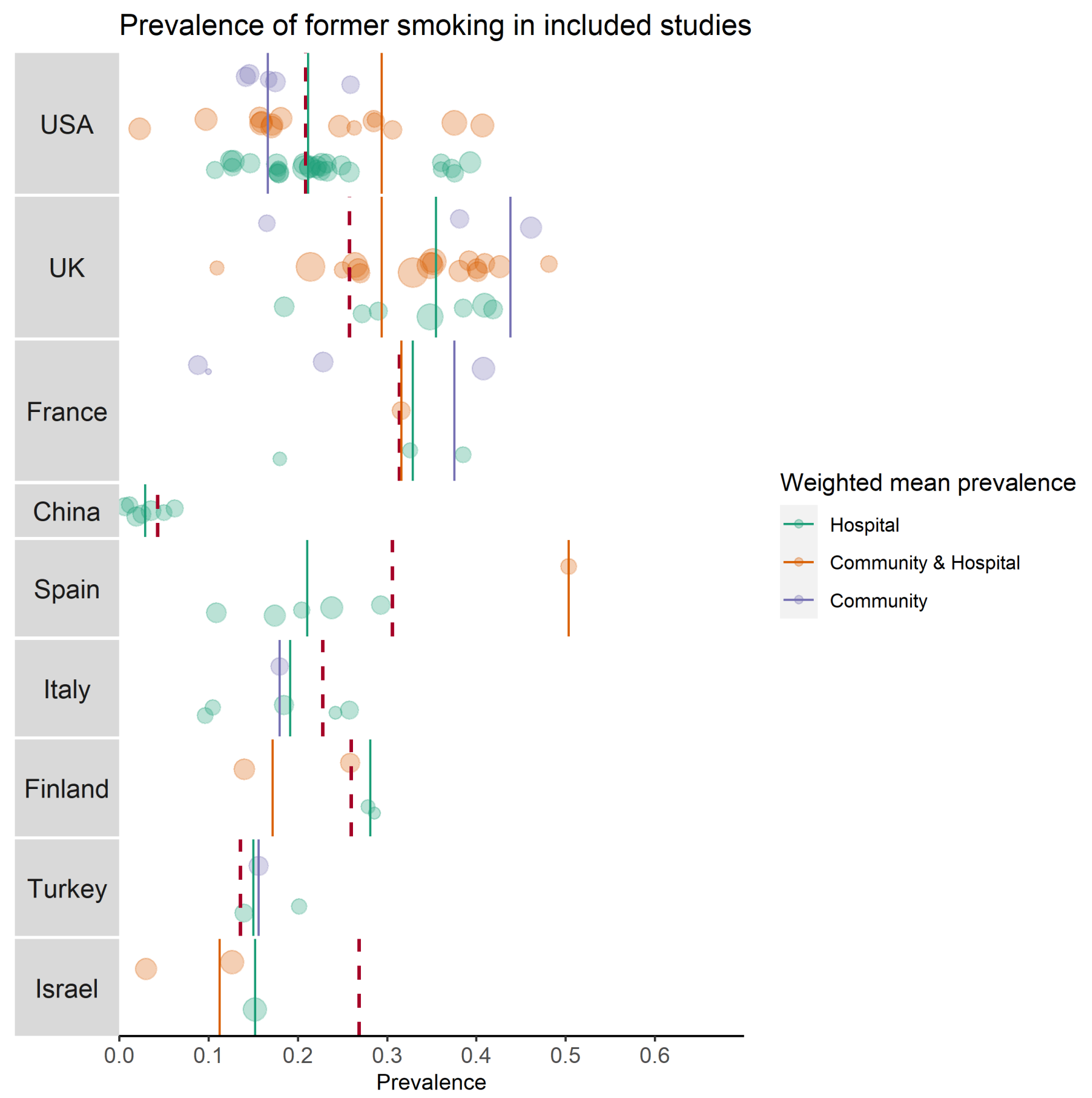

Figure 2b. Prevalence of former smoking in included studies, split by country (solid lines). The circles represent individual studies, with colour corresponding to study setting (i.e. community, community and hospital, hospital) and size corresponding to relative study sample size. For comparison, national former smoking prevalence is shown by dashed red lines. Countries with three or more eligible studies are shown.

SARS-CoV-2 testing by smoking status

Four studies provided data on access to SARS-CoV-2 diagnostic testing for those meeting local testing criteria by smoking status. In a cohort study of US military veterans aged 54-75 (Rentsch et al., 2020), current smokers were more likely to receive a test: $42.3 \%(1,603 / 3,789)$ of the sample were current smokers compared with $23.8 \%$ of all veterans aged $50+$ 
years using any tobacco product between 2010-2015 (Odani, 2018). In the UK Biobank cohort (Niedzwiedz et al., 2020), a multivariable analysis showed former $(\mathrm{RR}=1.29,95 \% \mathrm{Cl}=1.14-1.45, p<.001)$ and current $(\mathrm{RR}=1.44,95 \% \mathrm{Cl}=1.20$ $1.71, p<.001)$ compared with never smokers to be more likely to receive a test. In an Australian rapid assessment screening clinic for COVID-19 (Trubiano et al., 2020), 9.4\% (397/4,226) of the self-referred sample (subsequently assessed by a healthcare professional to decide on testing) were current smokers. Of these self-referrals, healthcare professionals decided that current compared with former or never smokers were less likely to require a test $(\mathrm{RR}=0.93,95 \% \mathrm{Cl}=0.86$ $1.0, p=0.045)$. In a further study using the UK Biobank cohort (Didikoglu et al., 2021), current $(R R=1.23,95 \% \mathrm{Cl}=1.19$ $1.26, p<0.001)$ and former smokers $(\mathrm{RR}=1.20,95 \% \mathrm{Cl}=1.18-1.23, p<0.001)$ were more likely to receive a test compared with never smokers.

\section{SARS-CoV-2 infection by smoking status}

76 studies provided data on SARS-CoV-2 infection for people meeting local testing criteria by smoking status (see Table 2). Meta-analyses were performed for 3 'good' and 27 'fair' quality studies (see Figure 3 and 4). Current smokers were at reduced risk of testing positive for SARS-CoV-2 compared with never smokers $(\mathrm{RR}=0.71,95 \%$ Credible Interval $(\mathrm{Crl})=$ $0.61-0.82, \tau=0.34)$. The three good quality studies each reported point estimates less than 1 , although the Crl for one of the three studies crossed 1 . The probability of current smokers being at reduced risk of infection compared with never smokers ( $R R \leq 0.9)$ was $>99 \%$. Former compared with never smokers were at increased risk of testing positive, but data were inconclusive $(R R=1.03,95 \% \mathrm{Crl}=0.95-1.11, \tau=0.17)$ and favoured there being no important association. The probability of former smokers being at increased risk of infection ( $R R \geq 1.1)$ compared with never smokers was $4 \%$. Results were materially unchanged in sensitivity analyses.

Table 2 SARS-CoV-2 infection by smoking status

\begin{tabular}{|c|c|c|c|c|c|c|c|c|c|c|c|}
\hline \multirow[b]{2}{*}{ Author } & \multirow[b]{2}{*}{$\begin{array}{l}\text { Total } \\
\text { population } \\
\text { tested }\end{array}$} & \multicolumn{6}{|c|}{ SARS-CoV-2 negative } & \multicolumn{4}{|c|}{ SARS-CoV-2 positive } \\
\hline & & $\mathbf{N}(\%)$ & $\begin{array}{l}\text { Current } \\
\text { smoker } \\
(\%)\end{array}$ & $\begin{array}{l}\text { Former } \\
\text { smoker } \\
(\%)\end{array}$ & $\begin{array}{l}\text { Current/former } \\
\text { smoker (\%) }\end{array}$ & $\begin{array}{l}\text { Never } \\
\text { smoker } \\
(\%)\end{array}$ & $\begin{array}{l}\text { Not } \\
\text { stated } \\
(\%)\end{array}$ & N (\%) & $\begin{array}{l}\text { Current } \\
\text { smoker } \\
(\%)\end{array}$ & $\begin{array}{l}\text { Former } \\
\text { smoker } \\
(\%)\end{array}$ & $\begin{array}{l}\text { Current/form } \\
\text { smoker (\%) }\end{array}$ \\
\hline Rentsch & 3,528 & $\begin{array}{l}2974 \\
(84.30 \%)\end{array}$ & $\begin{array}{l}1444 \\
(48.55 \%)\end{array}$ & $\begin{array}{l}704 \\
(23.67 \%)\end{array}$ & - & $\begin{array}{l}826 \\
(27.77 \%)\end{array}$ & - & $\begin{array}{l}554 \\
(15.70 \%)\end{array}$ & $\begin{array}{l}159 \\
(28.70 \%)\end{array}$ & $\begin{array}{l}179 \\
(32.31 \%)\end{array}$ & - \\
\hline Fontanet & 661 & $\begin{array}{l}490 \\
(74.13 \%)\end{array}$ & $\begin{array}{l}64 \\
(13.06 \%)\end{array}$ & - & - & $\begin{array}{l}426 \\
(86.94 \%)\end{array}$ & - & $\begin{array}{l}171 \\
(25.87 \%)\end{array}$ & $\begin{array}{l}5 \\
(2.92 \%)\end{array}$ & - & - \\
\hline Cho & 1,331 & $\begin{array}{l}793 \\
(59.58 \%)\end{array}$ & $\begin{array}{l}142 \\
(17.91 \%)\end{array}$ & $\begin{array}{l}214 \\
(26.99 \%)\end{array}$ & - & $\begin{array}{l}437 \\
(55.11 \%)\end{array}$ & - & $\begin{array}{l}538 \\
(40.42 \%)\end{array}$ & $\begin{array}{l}111 \\
(20.63 \%)\end{array}$ & $\begin{array}{l}145 \\
(26.95 \%)\end{array}$ & - \\
\hline Shah & 243 & $\begin{array}{l}212 \\
(87.24 \%)\end{array}$ & $\begin{array}{l}52 \\
(24.53 \%)\end{array}$ & $\begin{array}{l}47 \\
(22.17 \%)\end{array}$ & - & $\begin{array}{l}113 \\
(53.30 \%)\end{array}$ & - & $\begin{array}{l}29 \\
(11.93 \%)\end{array}$ & $\begin{array}{l}0 \\
(0.00 \%)\end{array}$ & $\begin{array}{l}9 \\
(31.03 \%)\end{array}$ & - \\
\hline Kolin & 1,474 & $\begin{array}{l}805 \\
(54.61 \%)\end{array}$ & $\begin{array}{l}141 \\
(17.52 \%)\end{array}$ & $\begin{array}{l}307 \\
(38.14 \%)\end{array}$ & - & $\begin{array}{l}354 \\
(43.98 \%)\end{array}$ & $3(0.37 \%)$ & $\begin{array}{l}669 \\
(45.39 \%)\end{array}$ & $\begin{array}{l}72 \\
(10.76 \%)\end{array}$ & $\begin{array}{l}285 \\
(42.60 \%)\end{array}$ & - \\
\hline de Lusignan & 3,291 & $\begin{array}{l}2740 \\
(83.26 \%)\end{array}$ & $\begin{array}{l}366 \\
(13.36 \%)\end{array}$ & $\begin{array}{l}1450 \\
(52.92 \%)\end{array}$ & - & $\begin{array}{l}924 \\
(33.72 \%)\end{array}$ & - & $\begin{array}{l}551 \\
(16.74 \%)\end{array}$ & $\begin{array}{l}47 \\
(8.53 \%)\end{array}$ & $\begin{array}{l}303 \\
(54.99 \%)\end{array}$ & - \\
\hline \multirow[t]{2}{*}{ Valenti } & 789 & $\begin{array}{l}689 \\
(87.33 \%)\end{array}$ & $\begin{array}{l}197 \\
(28.59 \%)\end{array}$ & - & - & - & $\begin{array}{l}492 \\
(71.41 \%)\end{array}$ & $\begin{array}{l}40 \\
(5.07 \%)\end{array}$ & $\begin{array}{l}7 \\
(17.50 \%)\end{array}$ & - & - \\
\hline & & on & 1 & in & & תד & & 27 & 1 & in & \\
\hline
\end{tabular}




\begin{tabular}{|c|c|c|c|c|c|c|c|c|c|c|c|}
\hline Parrotta & 76 & $\begin{array}{l}\text { งy } \\
\text { (51.32\%) }\end{array}$ & $\begin{array}{l}\text { ' } \\
(2.56 \%)\end{array}$ & $\begin{array}{l}\text { IU } \\
(25.64 \%)\end{array}$ & - & $\begin{array}{l}<1 \\
(69.23 \%)\end{array}$ & $1(2.56 \%)$ & (48.68\%) & $\begin{array}{l}\text { ' } \\
(2.70 \%)\end{array}$ & $\begin{array}{l}\text { IU } \\
(27.03 \%)\end{array}$ & - \\
\hline Berumen & 102,875 & $\begin{array}{l}71353 \\
(69.36 \%)\end{array}$ & - & - & $7173(10.05 \%)$ & $\begin{array}{l}64180 \\
(89.95 \%)\end{array}$ & - & $\begin{array}{l}31522 \\
(30.64 \%)\end{array}$ & - & - & 2748 (8.72\%) \\
\hline Israel & 24,906 & $\begin{array}{l}20755 \\
(83.33 \%)\end{array}$ & $\begin{array}{l}3783 \\
(18.23 \%)\end{array}$ & $\begin{array}{l}2671 \\
(12.87 \%)\end{array}$ & - & $\begin{array}{l}14301 \\
(68.90 \%)\end{array}$ & - & $\begin{array}{l}4151 \\
(16.67 \%)\end{array}$ & $\begin{array}{l}406 \\
(9.78 \%)\end{array}$ & $\begin{array}{l}483 \\
(11.64 \%)\end{array}$ & - \\
\hline Romao & 34 & $\begin{array}{l}20 \\
(58.82 \%)\end{array}$ & - & - & $5(25.00 \%)$ & - & $\begin{array}{l}15 \\
(75.00 \%)\end{array}$ & $\begin{array}{l}14 \\
(41.18 \%)\end{array}$ & - & - & $4(28.57 \%)$ \\
\hline Ramlall & 11,116 & $\begin{array}{l}4723 \\
(42.49 \%)\end{array}$ & - & - & - & - & - & $\begin{array}{l}6393 \\
(57.51 \%)\end{array}$ & - & - & $\begin{array}{l}1643.001 \\
(25.70 \%)\end{array}$ \\
\hline Eugen-Olsen & 407 & $\begin{array}{l}290 \\
(71.25 \%)\end{array}$ & $\begin{array}{l}76 \\
(26.21 \%)\end{array}$ & $\begin{array}{l}104 \\
(35.86 \%)\end{array}$ & - & $\begin{array}{l}102 \\
(35.17 \%)\end{array}$ & - & $\begin{array}{l}117 \\
(28.75 \%)\end{array}$ & $\begin{array}{l}8 \\
(6.84 \%)\end{array}$ & $\begin{array}{l}46 \\
(39.32 \%)\end{array}$ & - \\
\hline Raisi-Estabragh & 4,510 & $\begin{array}{l}3184 \\
(70.60 \%)\end{array}$ & - & - & $1653(51.92 \%)$ & - & $\begin{array}{l}1531 \\
(48.08 \%)\end{array}$ & $\begin{array}{l}1326 \\
(29.40 \%)\end{array}$ & - & - & $683(51.51 \%)$ \\
\hline Houlihan & 177 & $\begin{array}{l}97 \\
(54.80 \%)\end{array}$ & $\begin{array}{l}14 \\
(14.43 \%)\end{array}$ & $\begin{array}{l}14 \\
(14.43 \%)\end{array}$ & - & $\begin{array}{l}69 \\
(71.13 \%)\end{array}$ & - & $\begin{array}{l}80 \\
(45.20 \%)\end{array}$ & $\begin{array}{l}7 \\
(8.75 \%)\end{array}$ & $\begin{array}{l}19 \\
(23.75 \%)\end{array}$ & - \\
\hline McQueenie & 428,199 & $\begin{array}{l}424355 \\
(99.10 \%)\end{array}$ & - & - & $\begin{array}{l}189299 \\
(44.61 \%)\end{array}$ & $\begin{array}{l}235056 \\
(55.39 \%)\end{array}$ & - & $\begin{array}{l}1311 \\
(0.31 \%)\end{array}$ & - & - & 669 (51.03\%) \\
\hline Woolford & 4,474 & $\begin{array}{l}3161 \\
(70.65 \%)\end{array}$ & $\begin{array}{l}441 \\
(13.95 \%)\end{array}$ & $\begin{array}{l}1194 \\
(37.77 \%)\end{array}$ & - & $\begin{array}{l}1526 \\
(48.28 \%)\end{array}$ & - & $\begin{array}{l}1313 \\
(29.35 \%)\end{array}$ & $\begin{array}{l}145 \\
(11.04 \%)\end{array}$ & $\begin{array}{l}525 \\
(39.98 \%)\end{array}$ & - \\
\hline $\begin{array}{l}\text { Hernandez, } \\
\text { Garduno }\end{array}$ & 32,583 & $\begin{array}{l}20279 \\
(62.24 \%)\end{array}$ & - & - & $2399(11.83 \%)$ & $\begin{array}{l}17861 \\
(88.08 \%)\end{array}$ & - & $\begin{array}{l}12304 \\
(37.76 \%)\end{array}$ & - & - & $1191(9.68 \%)$ \\
\hline Govind & 6,215 & $\begin{array}{l}6207 \\
(99.87 \%)\end{array}$ & $\begin{array}{l}4104 \\
(66.12 \%)\end{array}$ & $\begin{array}{l}1669 \\
(26.89 \%)\end{array}$ & - & $\begin{array}{l}342 \\
(5.51 \%)\end{array}$ & - & $\begin{array}{l}102 \\
(1.64 \%)\end{array}$ & $\begin{array}{l}78 \\
(76.47 \%)\end{array}$ & $\begin{array}{l}20 \\
(19.61 \%)\end{array}$ & - \\
\hline $\mathrm{Gu}$ & 4,699 & $\begin{array}{l}3815 \\
(81.19 \%)\end{array}$ & $\begin{array}{l}360 \\
(9.44 \%)\end{array}$ & $\begin{array}{l}1142 \\
(29.93 \%)\end{array}$ & - & $\begin{array}{l}2313 \\
(60.63 \%)\end{array}$ & - & $\begin{array}{l}884 \\
(18.81 \%)\end{array}$ & $\begin{array}{l}40 \\
(4.52 \%)\end{array}$ & $\begin{array}{l}264 \\
(29.86 \%)\end{array}$ & - \\
\hline Kibler & 702 & $\begin{array}{l}680 \\
(96.87 \%)\end{array}$ & $\begin{array}{l}25 \\
(3.68 \%)\end{array}$ & - & - & - & $\begin{array}{l}655 \\
(96.32 \%)\end{array}$ & $\begin{array}{l}22 \\
(3.13 \%)\end{array}$ & $\begin{array}{l}1 \\
(4.55 \%)\end{array}$ & - & - \\
\hline Auvinen & 61 & $\begin{array}{l}33 \\
(54.10 \%)\end{array}$ & $\begin{array}{l}10 \\
(30.30 \%)\end{array}$ & $\begin{array}{l}8 \\
(24.24 \%)\end{array}$ & - & $\begin{array}{l}15 \\
(45.45 \%)\end{array}$ & - & $\begin{array}{l}28 \\
(45.90 \%)\end{array}$ & $\begin{array}{l}1 \\
(3.57 \%)\end{array}$ & $\begin{array}{l}9 \\
(32.14 \%)\end{array}$ & - \\
\hline Antonio-Villa & 34,263 & $\begin{array}{l}23338 \\
(68.11 \%)\end{array}$ & $\begin{array}{l}2293 \\
(9.83 \%)\end{array}$ & - & - & - & $\begin{array}{l}21045 \\
(90.17 \%)\end{array}$ & $\begin{array}{l}10925 \\
(31.89 \%)\end{array}$ & $\begin{array}{l}1023 \\
(9.36 \%)\end{array}$ & - & - \\
\hline Merzon & 7,807 & $\begin{array}{l}7025 \\
(89.98 \%)\end{array}$ & - & - & $1136(16.17 \%)$ & - & $\begin{array}{l}5889 \\
(83.83 \%)\end{array}$ & $\begin{array}{l}782 \\
(10.02 \%)\end{array}$ & - & - & $127(16.24 \%)$ \\
\hline Trubiano & 2,935 & $\begin{array}{l}2827 \\
(96.32 \%)\end{array}$ & - & - & 256 (9.06\%) & - & $\begin{array}{l}2586 \\
(91.48 \%)\end{array}$ & $\begin{array}{l}108 \\
(3.68 \%)\end{array}$ & - & - & 3 (2.78\%) \\
\hline Shi, Resurreccion & 1,521 & $\begin{array}{l}1265 \\
(83.17 \%)\end{array}$ & - & - & 681 (53.83\%) & - & $\begin{array}{l}584 \\
(46.17 \%)\end{array}$ & $\begin{array}{l}256 \\
(16.83 \%)\end{array}$ & - & - & $154(60.16 \%)$ \\
\hline Riley & 120,620 & $\begin{array}{l}120461 \\
(99.87 \%)\end{array}$ & $\begin{array}{l}2594 \\
(2.15 \%)\end{array}$ & - & - & $\begin{array}{l}19914 \\
(16.53 \%)\end{array}$ & $\begin{array}{l}97953 \\
(81.32 \%)\end{array}$ & $\begin{array}{l}159 \\
(0.13 \%)\end{array}$ & $\begin{array}{l}3 \\
(1.89 \%)\end{array}$ & - & - \\
\hline Alizadehsani & 319 & $\begin{array}{l}196 \\
(61.44 \%)\end{array}$ & - & - & - & - & $\begin{array}{l}196 \\
(100.00 \%)\end{array}$ & $\begin{array}{l}123 \\
(38.56 \%)\end{array}$ & - & - & $1(0.81 \%)$ \\
\hline Merkely & 10,474 & $\begin{array}{l}10336 \\
(98.68 \%)\end{array}$ & $\begin{array}{l}2904 \\
(28.10 \%)\end{array}$ & $\begin{array}{l}2107 \\
(20.39 \%)\end{array}$ & - & $\begin{array}{l}5310 \\
(51.37 \%)\end{array}$ & $\begin{array}{l}15 \\
(0.15 \%)\end{array}$ & $\begin{array}{l}70 \\
(0.67 \%)\end{array}$ & $\begin{array}{l}16 \\
(22.86 \%)\end{array}$ & $\begin{array}{l}15 \\
(21.43 \%)\end{array}$ & - \\
\hline
\end{tabular}




\begin{tabular}{|c|c|c|c|c|c|c|c|c|c|c|c|}
\hline$-\cdots \cdots+\cdots$ & $\cdots$ & (56.46\%) & & & $\cdots, 1-\cdots \cdots$, & & (73.73\%) & $(43.54 \%)$ & & & $-1, \cdots-\cdots$, \\
\hline Reiter & 235 & $\begin{array}{l}175 \\
(74.47 \%)\end{array}$ & - & - & 93 (53.14\%) & $\begin{array}{l}82 \\
(46.86 \%)\end{array}$ & - & $\begin{array}{l}60 \\
(25.53 \%)\end{array}$ & - & - & $13(21.67 \%)$ \\
\hline Izquierdo & 71,192 & $\begin{array}{l}\text { NA ( } \\
\text { NA\%) }\end{array}$ & - & - & - & - & - & $\begin{array}{l}1006 \\
(1.41 \%)\end{array}$ & $\begin{array}{l}111 \\
(11.03 \%)\end{array}$ & - & - \\
\hline Ward & 99,908 & $\begin{array}{l}94416 \\
(94.50 \%)\end{array}$ & $\begin{array}{l}10202 \\
(10.81 \%)\end{array}$ & - & - & - & $\begin{array}{l}84214 \\
(89.19 \%)\end{array}$ & $\begin{array}{l}5492 \\
(5.50 \%)\end{array}$ & $\begin{array}{l}433 \\
(7.88 \%)\end{array}$ & - & - \\
\hline Ebinger & 6,062 & $\begin{array}{l}5850 \\
(96.50 \%)\end{array}$ & $\begin{array}{l}99 \\
(1.69 \%)\end{array}$ & - & - & - & $\begin{array}{l}5668 \\
(96.89 \%)\end{array}$ & $\begin{array}{l}212 \\
(3.50 \%)\end{array}$ & $\begin{array}{l}3 \\
(1.42 \%)\end{array}$ & - & - \\
\hline Salerno & 15,920 & $\begin{array}{l}14753 \\
(92.67 \%)\end{array}$ & - & - & $5517(37.40 \%)$ & $\begin{array}{l}8278 \\
(56.11 \%)\end{array}$ & $\begin{array}{l}958 \\
(6.49 \%)\end{array}$ & $\begin{array}{l}1167 \\
(7.33 \%)\end{array}$ & - & - & $339(29.05 \%)$ \\
\hline Iversen & 28,792 & $\begin{array}{l}27629 \\
(95.96 \%)\end{array}$ & $\begin{array}{l}4430 \\
(16.03 \%)\end{array}$ & $\begin{array}{l}1799 \\
(6.51 \%)\end{array}$ & - & $\begin{array}{l}21217 \\
(76.79 \%)\end{array}$ & $\begin{array}{l}246 \\
(0.89 \%)\end{array}$ & $\begin{array}{l}1163 \\
(4.04 \%)\end{array}$ & $\begin{array}{l}177 \\
(15.22 \%)\end{array}$ & $\begin{array}{l}78 \\
(6.71 \%)\end{array}$ & - \\
\hline Hippisley-Cox & $8,275,949$ & $\begin{array}{l}\text { NA ( } \\
\text { NA\%) }\end{array}$ & - & - & - & - & - & $\begin{array}{l}19486 \\
(0.24 \%)\end{array}$ & $\begin{array}{l}1354 \\
(6.95 \%)\end{array}$ & $\begin{array}{l}5715 \\
(29.33 \%)\end{array}$ & - \\
\hline Fillmore & 22,914 & $\begin{array}{l}21120 \\
(92.17 \%)\end{array}$ & $\begin{array}{l}8137 \\
(38.53 \%)\end{array}$ & $\begin{array}{l}8416 \\
(39.85 \%)\end{array}$ & - & $\begin{array}{l}3227 \\
(15.28 \%)\end{array}$ & $\begin{array}{l}1340 \\
(6.34 \%)\end{array}$ & $\begin{array}{l}1794 \\
(7.83 \%)\end{array}$ & $\begin{array}{l}452 \\
(25.20 \%)\end{array}$ & $\begin{array}{l}899 \\
(50.11 \%)\end{array}$ & - \\
\hline Alkurt & 119 & $\begin{array}{l}\text { NA ( } \\
\text { NA\%) }\end{array}$ & - & - & - & - & - & $\begin{array}{l}119 \\
(100.00 \%)\end{array}$ & $\begin{array}{l}14 \\
(11.76 \%)\end{array}$ & - & - \\
\hline Petrilli & 10,620 & $\begin{array}{l}5341 \\
(50.29 \%)\end{array}$ & $\begin{array}{l}3454 \\
(64.67 \%)\end{array}$ & $\begin{array}{l}816 \\
(15.28 \%)\end{array}$ & - & $\begin{array}{l}541 \\
(10.13 \%)\end{array}$ & $\begin{array}{l}530 \\
(9.92 \%)\end{array}$ & $\begin{array}{l}5279 \\
(49.71 \%)\end{array}$ & $\begin{array}{l}3268 \\
(61.91 \%)\end{array}$ & $\begin{array}{l}902 \\
(17.09 \%)\end{array}$ & - \\
\hline Bello-Chavolla & 150,200 & $\begin{array}{l}98567 \\
(65.62 \%)\end{array}$ & - & - & 9624 (9.76\%) & - & $\begin{array}{l}88943 \\
(90.24 \%)\end{array}$ & $\begin{array}{l}51633 \\
(34.38 \%)\end{array}$ & - & - & $4366(8.46 \%)$ \\
\hline Ariza & 351 & $\begin{array}{l}322 \\
(91.74 \%)\end{array}$ & $\begin{array}{l}21 \\
(6.52 \%)\end{array}$ & - & - & - & $\begin{array}{l}301 \\
(93.48 \%)\end{array}$ & $\begin{array}{l}29 \\
(8.26 \%)\end{array}$ & $\begin{array}{l}3 \\
(10.34 \%)\end{array}$ & - & - \\
\hline Meini & 461 & $\begin{array}{l}243 \\
(52.71 \%)\end{array}$ & $\begin{array}{l}39 \\
(16.05 \%)\end{array}$ & $\begin{array}{l}66 \\
(27.16 \%)\end{array}$ & - & $\begin{array}{l}138 \\
(56.79 \%)\end{array}$ & - & $\begin{array}{l}218 \\
(47.29 \%)\end{array}$ & $\begin{array}{l}9 \\
(4.13 \%)\end{array}$ & $\begin{array}{l}53 \\
(24.31 \%)\end{array}$ & - \\
\hline Favara & 434 & $\begin{array}{l}354 \\
(81.57 \%)\end{array}$ & $\begin{array}{l}28 \\
(7.91 \%)\end{array}$ & - & - & - & $\begin{array}{l}326 \\
(92.09 \%)\end{array}$ & $\begin{array}{l}80 \\
(18.43 \%)\end{array}$ & $\begin{array}{l}9 \\
(11.25 \%)\end{array}$ & - & - \\
\hline Erber & 4,554 & $\begin{array}{l}4446 \\
(97.63 \%)\end{array}$ & - & - & 806 (18.13\%) & - & $\begin{array}{l}3640 \\
(81.87 \%)\end{array}$ & $\begin{array}{l}108 \\
(2.37 \%)\end{array}$ & - & - & $11(10.19 \%)$ \\
\hline Roederer & 815 & $\begin{array}{l}390 \\
(47.85 \%)\end{array}$ & $\begin{array}{l}175 \\
(44.87 \%)\end{array}$ & $\begin{array}{l}32 \\
(8.21 \%)\end{array}$ & - & $\begin{array}{l}183 \\
(46.92 \%)\end{array}$ & - & $\begin{array}{l}425 \\
(52.15 \%)\end{array}$ & $\begin{array}{l}127 \\
(29.88 \%)\end{array}$ & $\begin{array}{l}40 \\
(9.41 \%)\end{array}$ & - \\
\hline Makaronidis & 567 & $\begin{array}{l}127 \\
(22.40 \%)\end{array}$ & $\begin{array}{l}16 \\
(12.60 \%)\end{array}$ & - & - & - & $\begin{array}{l}111 \\
(87.40 \%)\end{array}$ & $\begin{array}{l}440 \\
(77.60 \%)\end{array}$ & $\begin{array}{l}37 \\
(8.41 \%)\end{array}$ & - & - \\
\hline loannou & 88,747 & $\begin{array}{l}78616 \\
(88.58 \%)\end{array}$ & $\begin{array}{l}17138 \\
(21.80 \%)\end{array}$ & $\begin{array}{l}29245 \\
(37.20 \%)\end{array}$ & - & $\begin{array}{l}22327 \\
(28.40 \%)\end{array}$ & $\begin{array}{l}9906 \\
(12.60 \%)\end{array}$ & $\begin{array}{l}10131 \\
(11.42 \%)\end{array}$ & $\begin{array}{l}1135 \\
(11.20 \%)\end{array}$ & $\begin{array}{l}4073 \\
(40.20 \%)\end{array}$ & - \\
\hline Perico & 423 & $\begin{array}{l}260 \\
(61.47 \%)\end{array}$ & $\begin{array}{l}69 \\
(26.54 \%)\end{array}$ & $\begin{array}{l}35 \\
(13.46 \%)\end{array}$ & - & $\begin{array}{l}156 \\
(60.00 \%)\end{array}$ & - & $\begin{array}{l}163 \\
(38.53 \%)\end{array}$ & $\begin{array}{l}23 \\
(14.11 \%)\end{array}$ & $\begin{array}{l}41 \\
(25.15 \%)\end{array}$ & - \\
\hline Vila-Corcoles & 2,324 & $\begin{array}{l}1944 \\
(83.65 \%)\end{array}$ & - & - & - & - & - & $\begin{array}{l}380 \\
(16.35 \%)\end{array}$ & - & - & 27 (7.11\%) \\
\hline O'Reilly & 1,334 & $\begin{array}{l}1284 \\
(96.25 \%)\end{array}$ & - & - & $376(29.28 \%)$ & - & $\begin{array}{l}908 \\
(70.72 \%)\end{array}$ & $\begin{array}{l}50 \\
(3.75 \%)\end{array}$ & - & - & $4(8.00 \%)$ \\
\hline Ghinai & 1,435 & $\begin{array}{l}1004 \\
(69.97 \%)\end{array}$ & $\begin{array}{l}412 \\
(41.04 \%)\end{array}$ & $\begin{array}{l}155 \\
(15.44 \%)\end{array}$ & - & $\begin{array}{l}341 \\
(33.96 \%)\end{array}$ & $\begin{array}{l}96 \\
(9.56 \%)\end{array}$ & $\begin{array}{l}431 \\
(30.03 \%)\end{array}$ & $\begin{array}{l}113 \\
(26.22 \%)\end{array}$ & $\begin{array}{l}96 \\
(22.27 \%)\end{array}$ & - \\
\hline Kortela & 2,993 & $\begin{array}{l}2419 \\
(80.82 \%)\end{array}$ & $\begin{array}{l}300 \\
(12.40 \%)\end{array}$ & $\begin{array}{l}340 \\
(14.06 \%)\end{array}$ & - & $\begin{array}{l}636 \\
(26.29 \%)\end{array}$ & $\begin{array}{l}1143 \\
(47.25 \%)\end{array}$ & $\begin{array}{l}574 \\
(19.18 \%)\end{array}$ & $\begin{array}{l}26 \\
(4.53 \%)\end{array}$ & $\begin{array}{l}80 \\
(13.94 \%)\end{array}$ & - \\
\hline
\end{tabular}




\begin{tabular}{|c|c|c|c|c|c|c|c|c|c|c|c|}
\hline Saeed & 173 & $\begin{array}{l}69 \\
(39.88 \%)\end{array}$ & $\begin{array}{l}2 \\
(2.90 \%)\end{array}$ & - & - & - & $\begin{array}{l}67 \\
(97.10 \%)\end{array}$ & $\begin{array}{l}104 \\
(60.12 \%)\end{array}$ & $\begin{array}{l}9 \\
(8.65 \%)\end{array}$ & - & - \\
\hline Woolcott & $1,636,050$ & $\begin{array}{l}878840 \\
(53.72 \%)\end{array}$ & - & - & $85816(9.76 \%)$ & - & $\begin{array}{l}793024 \\
(90.24 \%)\end{array}$ & $\begin{array}{l}757210 \\
(46.28 \%)\end{array}$ & - & - & 57451 (7.59\% \\
\hline Barasa & 394 & $\begin{array}{l}277 \\
(70.30 \%)\end{array}$ & $\begin{array}{l}49 \\
(17.69 \%)\end{array}$ & $\begin{array}{l}105 \\
(37.91 \%)\end{array}$ & - & $\begin{array}{l}110 \\
(39.71 \%)\end{array}$ & $\begin{array}{l}13 \\
(4.69 \%)\end{array}$ & $\begin{array}{l}117 \\
(29.70 \%)\end{array}$ & $\begin{array}{l}9 \\
(7.69 \%)\end{array}$ & $\begin{array}{l}37 \\
(31.62 \%)\end{array}$ & - \\
\hline Paleiron & 1,688 & $\begin{array}{l}409 \\
(24.23 \%)\end{array}$ & $\begin{array}{l}236 \\
(57.70 \%)\end{array}$ & $\begin{array}{l}77 \\
(18.83 \%)\end{array}$ & - & $\begin{array}{l}96 \\
(23.47 \%)\end{array}$ & - & $\begin{array}{l}1279 \\
(75.77 \%)\end{array}$ & $\begin{array}{l}579 \\
(45.27 \%)\end{array}$ & $\begin{array}{l}309 \\
(24.16 \%)\end{array}$ & - \\
\hline Didikoglu & 43,428 & $\begin{array}{l}35695 \\
(82.19 \%)\end{array}$ & $\begin{array}{l}3919 \\
(10.98 \%)\end{array}$ & $\begin{array}{l}13841 \\
(38.78 \%)\end{array}$ & - & $\begin{array}{l}17939 \\
(50.26 \%)\end{array}$ & - & $\begin{array}{l}7733 \\
(17.81 \%)\end{array}$ & $\begin{array}{l}867 \\
(11.21 \%)\end{array}$ & $\begin{array}{l}2966 \\
(38.36 \%)\end{array}$ & - \\
\hline Kantele & 1,095 & $\begin{array}{l}1059 \\
(96.71 \%)\end{array}$ & $\begin{array}{l}176 \\
(16.62 \%)\end{array}$ & $\begin{array}{l}272 \\
(25.68 \%)\end{array}$ & - & $\begin{array}{l}611 \\
(57.70 \%)\end{array}$ & - & $\begin{array}{l}36 \\
(3.29 \%)\end{array}$ & $\begin{array}{l}3 \\
(8.33 \%)\end{array}$ & $\begin{array}{l}11 \\
(30.56 \%)\end{array}$ & - \\
\hline Polat & 1,322 & $\begin{array}{l}\text { NA ( } \\
\text { NA\%) }\end{array}$ & - & - & - & - & - & $\begin{array}{l}23 \\
(1.74 \%)\end{array}$ & $\begin{array}{l}4 \\
(17.39 \%)\end{array}$ & $\begin{array}{l}2 \\
(8.70 \%)\end{array}$ & - \\
\hline Richard & 8,344 & $\begin{array}{l}6798 \\
(81.47 \%)\end{array}$ & $\begin{array}{l}1065 \\
(15.67 \%)\end{array}$ & $\begin{array}{l}1171 \\
(17.23 \%)\end{array}$ & - & $\begin{array}{l}4456 \\
(65.55 \%)\end{array}$ & $\begin{array}{l}106 \\
(1.56 \%)\end{array}$ & $\begin{array}{l}531 \\
(6.36 \%)\end{array}$ & $\begin{array}{l}47 \\
(8.85 \%)\end{array}$ & $\begin{array}{l}83 \\
(15.63 \%)\end{array}$ & - \\
\hline Schubl & 1,557 & $\begin{array}{l}1392 \\
(89.40 \%)\end{array}$ & $\begin{array}{l}33 \\
(2.37 \%)\end{array}$ & - & - & - & $\begin{array}{l}1359 \\
(97.63 \%)\end{array}$ & $\begin{array}{l}165 \\
(10.60 \%)\end{array}$ & $\begin{array}{l}4 \\
(2.42 \%)\end{array}$ & - & - \\
\hline Nezhadmoghadam & 33,325 & $\begin{array}{l}19958 \\
(59.89 \%)\end{array}$ & $\begin{array}{l}2151 \\
(10.78 \%)\end{array}$ & & - & - & $\begin{array}{l}17807 \\
(89.22 \%)\end{array}$ & $\begin{array}{l}13367 \\
(40.11 \%)\end{array}$ & $\begin{array}{l}1251 \\
(9.36 \%)\end{array}$ & - & - \\
\hline Mora & 1,058 & $\begin{array}{l}857 \\
(81.00 \%)\end{array}$ & $\begin{array}{l}40 \\
(4.67 \%)\end{array}$ & $\begin{array}{l}118 \\
(13.77 \%)\end{array}$ & - & $\begin{array}{l}698 \\
(81.45 \%)\end{array}$ & $1(0.12 \%)$ & $\begin{array}{l}201 \\
(19.00 \%)\end{array}$ & $\begin{array}{l}8 \\
(3.98 \%)\end{array}$ & $\begin{array}{l}36 \\
(17.91 \%)\end{array}$ & - \\
\hline Molenaar & 696 & $\begin{array}{l}591 \\
(84.91 \%)\end{array}$ & $\begin{array}{l}29 \\
(4.91 \%)\end{array}$ & - & - & - & $\begin{array}{l}562 \\
(95.09 \%)\end{array}$ & $\begin{array}{l}105 \\
(15.09 \%)\end{array}$ & $\begin{array}{l}2 \\
(1.90 \%)\end{array}$ & - & - \\
\hline $\begin{array}{l}\text { Vila-Corcoles, } \\
\text { Satue-Gracia, Vila- } \\
\text { Rovira }\end{array}$ & 4,113 & $\begin{array}{l}3577 \\
(86.97 \%)\end{array}$ & $\begin{array}{l}591 \\
(16.52 \%)\end{array}$ & - & - & - & $\begin{array}{l}2986 \\
(83.48 \%)\end{array}$ & $\begin{array}{l}536 \\
(13.03 \%)\end{array}$ & $\begin{array}{l}41 \\
(7.65 \%)\end{array}$ & - & - \\
\hline Gegout Petit & 2,006 & $\begin{array}{l}1964 \\
(97.91 \%)\end{array}$ & $\begin{array}{l}334 \\
(17.01 \%)\end{array}$ & - & - & $\begin{array}{l}1545 \\
(78.67 \%)\end{array}$ & $\begin{array}{l}85 \\
(4.33 \%)\end{array}$ & $\begin{array}{l}42 \\
(2.09 \%)\end{array}$ & $\begin{array}{l}4 \\
(9.52 \%)\end{array}$ & - & - \\
\hline Leister & 3,301 & $\begin{array}{l}3269 \\
(99.03 \%)\end{array}$ & $\begin{array}{l}764 \\
(23.37 \%)\end{array}$ & - & - & - & $\begin{array}{l}2505 \\
(76.63 \%)\end{array}$ & $\begin{array}{l}32 \\
(0.97 \%)\end{array}$ & $\begin{array}{l}5 \\
(15.62 \%)\end{array}$ & - & - \\
\hline & & & & & & & & & & & - \\
\hline
\end{tabular}


Forest plot of current smokers and risk of testing positive

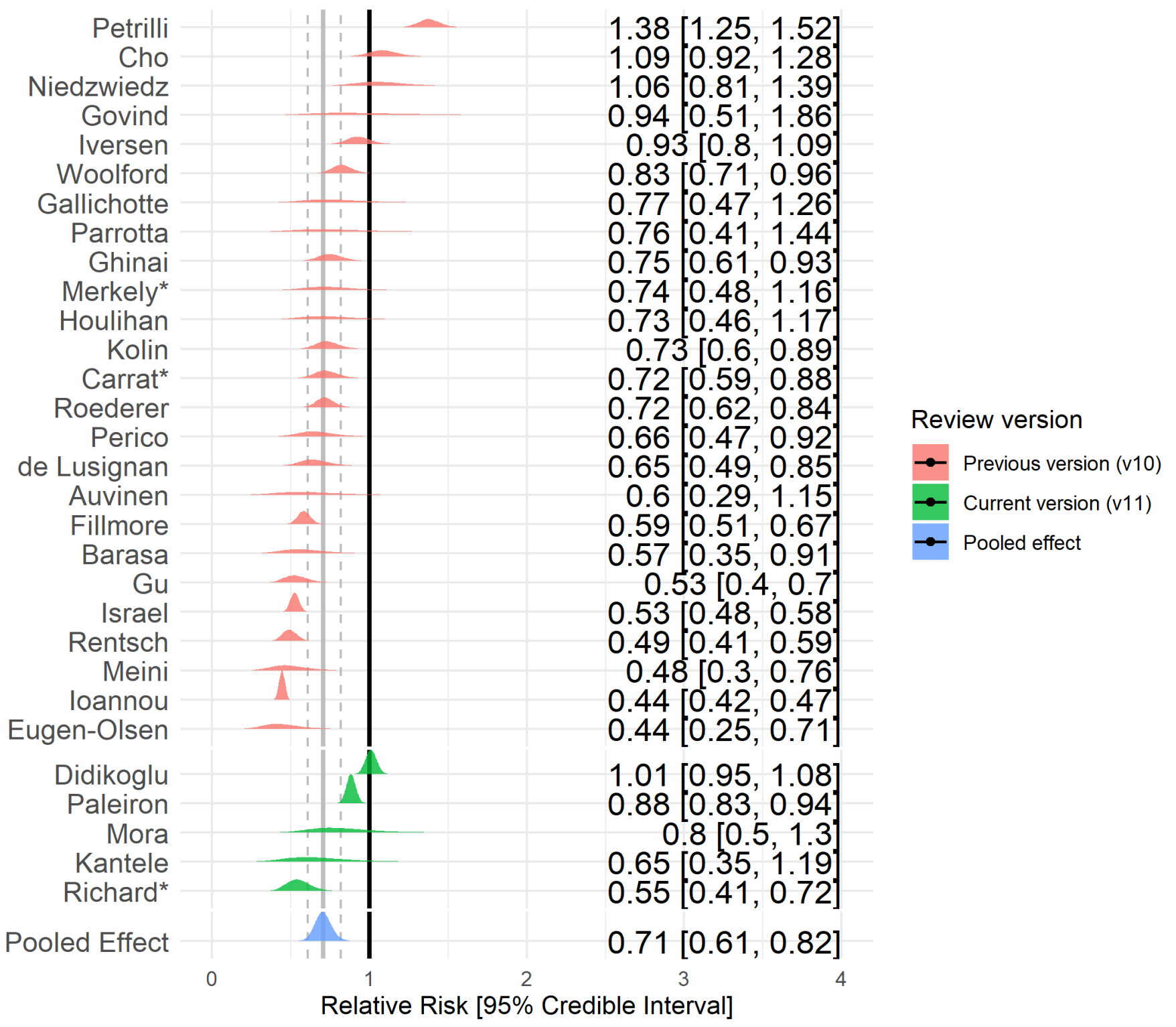

v10 testing prior

Figure 3. Forest plot for risk of testing positive for SARS-CoV-2 in current vs. never smokers. * Indicates 'good' quality studies. The prior from the previous review version ( $\mathrm{v} 10)$ was $\mathrm{RR}=0.69$. 
Forest plot of former smokers and risk of testing positive

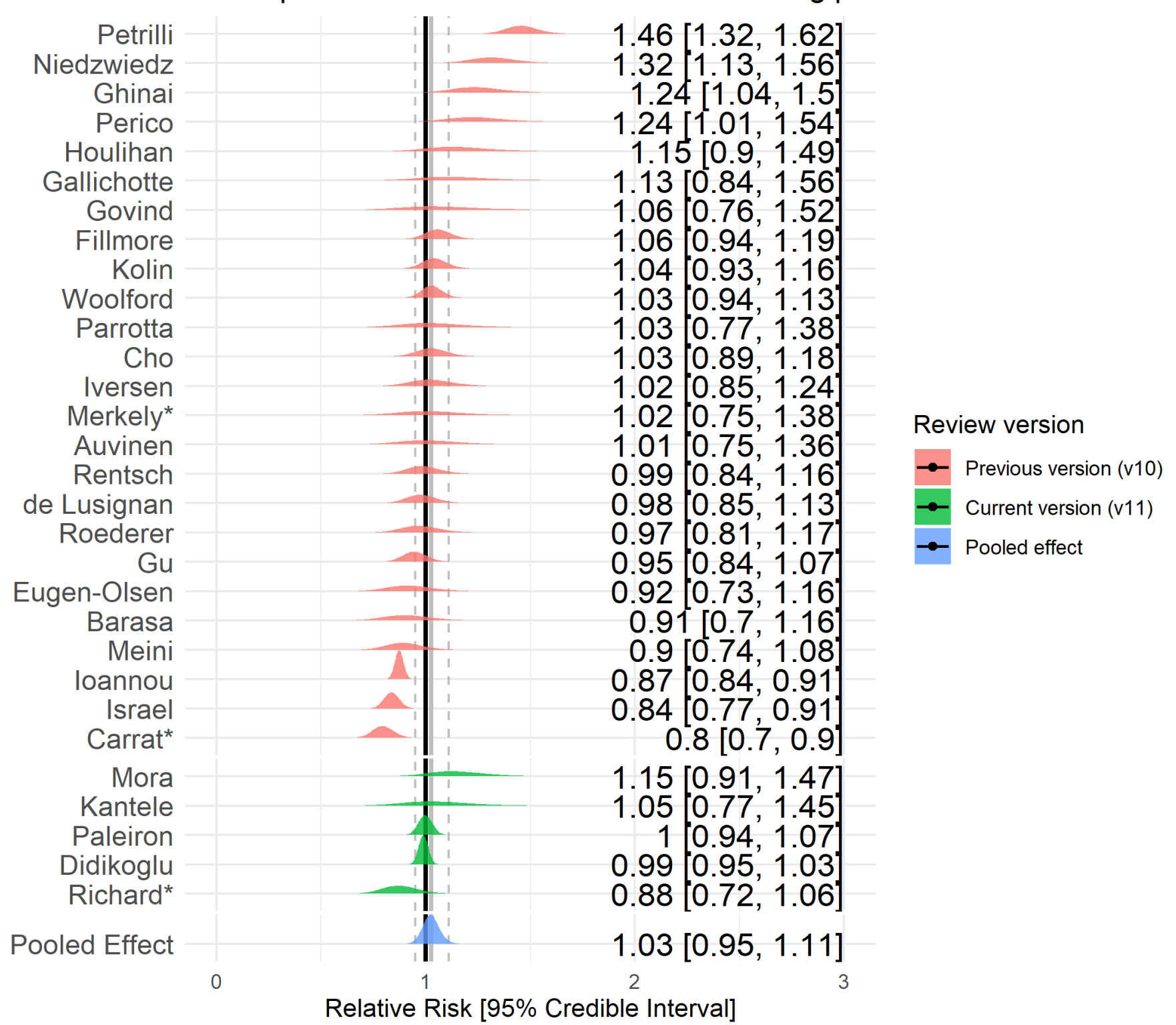

v10 testing prior

Figure 4. Forest plot for risk of testing positive for SARS-CoV-2 in former vs. never smokers. * Indicates 'good' quality studies. The prior from the previous review version ( 110$)$ was $\mathrm{RR}=1.02$.

Hospitalisation for COVID-19 by smoking status

48 studies examined hospitalisation for COVID-19 disease, stratified by smoking status (see Table 3). Meta-analyses were performed for 17 'fair' quality studies (see Figure 5 and 6$)$. Current $(R R=1.1,95 \% \mathrm{Crl}=0.99-1.21, \tau=0.15$ ) and former $(R R=1.19, \mathrm{Crl}=1.1-1.29, \tau=0.13)$ compared with never smokers were at increased risk of hospitalisation with COVID19. However, data for current smokers were inconclusive and favoured there being no important association. The probability of current and former smokers being at increased risk of hospitalisation ( $R R \geq 1.1)$ compared with never smokers was $49 \%$ and $98 \%$, respectively. Results were materially unchanged in two sensitivity analyses. 
Table 3 COVID-19 hospitalsation by smoking status

\begin{tabular}{|c|c|c|c|c|c|c|c|c|c|c|c|c|}
\hline \multirow[b]{2}{*}{ Author } & \multirow[b]{2}{*}{$\begin{array}{l}\text { Population } \\
\text { with } \\
\text { outcome }\end{array}$} & \multicolumn{7}{|c|}{ Community } & \multicolumn{4}{|c|}{ Hospitalised } \\
\hline & & N (\%) & $\begin{array}{l}\text { Current } \\
\text { smoker } \\
(\%)\end{array}$ & $\begin{array}{l}\text { Former } \\
\text { smoker } \\
(\%)\end{array}$ & $\begin{array}{l}\text { Current/former } \\
\text { smoker (\%) }\end{array}$ & $\begin{array}{l}\text { Never } \\
\text { smoker } \\
(\%)\end{array}$ & $\begin{array}{l}\text { Never/unknown } \\
\text { smoker (\%) }\end{array}$ & $\begin{array}{l}\text { Not } \\
\text { stated } \\
(\%)\end{array}$ & $\mathbf{N}(\%)$ & $\begin{array}{l}\text { Current } \\
\text { smoker } \\
(\%)\end{array}$ & $\begin{array}{l}\text { Former } \\
\text { smoker } \\
(\%)\end{array}$ & $\begin{array}{l}\text { Cur } \\
\text { sme }\end{array}$ \\
\hline Rentsch & 554 & $\begin{array}{l}269 \\
(48 \%)\end{array}$ & $\begin{array}{l}69 \\
(25.65 \%)\end{array}$ & $\begin{array}{l}90 \\
(33.46 \%)\end{array}$ & & $\begin{array}{l}110 \\
(40.89 \%)\end{array}$ & - & - & $\begin{array}{l}285 \\
(51 \%)\end{array}$ & $\begin{array}{l}90 \\
(31.58 \%)\end{array}$ & $\begin{array}{l}89 \\
(31.23 \%)\end{array}$ & - \\
\hline $\begin{array}{l}\text { Chow (US } \\
\text { CDC) }\end{array}$ & 6,637 & $\begin{array}{l}5143 \\
(77 \%)\end{array}$ & $\begin{array}{l}61 \\
(1.19 \%)\end{array}$ & $\begin{array}{l}80 \\
(1.56 \%)\end{array}$ & - & - & - & $\begin{array}{l}5002 \\
(97.26 \%)\end{array}$ & $\begin{array}{l}1494 \\
(22 \%)\end{array}$ & $\begin{array}{l}27 \\
(1.81 \%)\end{array}$ & $\begin{array}{l}78 \\
(5.22 \%)\end{array}$ & - \\
\hline Argenziano & 1,000 & $\begin{array}{l}151 \\
(15 \%)\end{array}$ & $\begin{array}{l}14 \\
(9.27 \%)\end{array}$ & $\begin{array}{l}18 \\
(11.92 \%)\end{array}$ & - & $\begin{array}{l}119 \\
(78.81 \%)\end{array}$ & - & - & $\begin{array}{l}849 \\
(84 \%)\end{array}$ & $\begin{array}{l}35 \\
(4.12 \%)\end{array}$ & $\begin{array}{l}161 \\
(18.96 \%)\end{array}$ & - \\
\hline Lubetzky & 54 & $\begin{array}{l}15 \\
(27 \%)\end{array}$ & - & - & $4(26.67 \%)$ & - & - & $\begin{array}{l}11 \\
(73.33 \%)\end{array}$ & $\begin{array}{l}39 \\
(72 \%)\end{array}$ & - & - & $8(2$ \\
\hline Carillo-Vega & 9,946 & $\begin{array}{l}3922 \\
(39 \%)\end{array}$ & $\begin{array}{l}408 \\
(10.40 \%)\end{array}$ & - & - & - & - & $\begin{array}{l}3514 \\
(89.60 \%)\end{array}$ & $\begin{array}{l}6024 \\
(60 \%)\end{array}$ & $\begin{array}{l}486 \\
(8.07 \%)\end{array}$ & - & - \\
\hline Yanover & 4,353 & $\begin{array}{l}4180 \\
(96 \%)\end{array}$ & $\begin{array}{l}484 \\
(11.58 \%)\end{array}$ & $\begin{array}{l}118 \\
(2.82 \%)\end{array}$ & - & $\begin{array}{l}3578 \\
(85.60 \%)\end{array}$ & - & - & $\begin{array}{l}173 \\
(3 \%)\end{array}$ & $\begin{array}{l}30 \\
(17.34 \%)\end{array}$ & $\begin{array}{l}11 \\
(6.36 \%)\end{array}$ & - \\
\hline Hamer & 387,109 & $\begin{array}{l}386349 \\
(99 \%)\end{array}$ & $\begin{array}{l}37333 \\
(9.66 \%)\end{array}$ & $\begin{array}{l}134542 \\
(34.82 \%)\end{array}$ & - & $\begin{array}{l}214474 \\
(55.51 \%)\end{array}$ & - & - & $\begin{array}{l}760 \\
(0 \%)\end{array}$ & $\begin{array}{l}93 \\
(12.24 \%)\end{array}$ & $\begin{array}{l}313 \\
(41.18 \%)\end{array}$ & - \\
\hline Heili-Frades & 4,712 & $\begin{array}{l}1973 \\
(41 \%)\end{array}$ & $\begin{array}{l}121 \\
(6.13 \%)\end{array}$ & $\begin{array}{l}222 \\
(11.25 \%)\end{array}$ & - & - & $1630(82.62 \%)$ & $\begin{array}{l}1630 \\
(82.62 \%)\end{array}$ & $\begin{array}{l}2739 \\
(58 \%)\end{array}$ & $\begin{array}{l}112 \\
(4.09 \%)\end{array}$ & $\begin{array}{l}598 \\
(21.83 \%)\end{array}$ & - \\
\hline Freites & 123 & $\begin{array}{l}69 \\
(56 \%)\end{array}$ & $\begin{array}{l}1 \\
(1.45 \%)\end{array}$ & - & - & - & - & $\begin{array}{l}68 \\
(98.55 \%)\end{array}$ & $\begin{array}{l}54 \\
(43 \%)\end{array}$ & $\begin{array}{l}3 \\
(5.56 \%)\end{array}$ & - & - \\
\hline Berumen & 102,875 & $\begin{array}{l}18832 \\
(18 \%)\end{array}$ & - & - & $1546(8.21 \%)$ & - & $17286(91.79 \%)$ & - & $\begin{array}{l}12690 \\
(12 \%)\end{array}$ & - & - & 120 \\
\hline Gianfrancesco & 600 & $\begin{array}{l}323 \\
(53 \%)\end{array}$ & - & - & $61(18.89 \%)$ & - & - & $\begin{array}{l}262 \\
(81.11 \%)\end{array}$ & $\begin{array}{l}277 \\
(46 \%)\end{array}$ & - & - & 68( \\
\hline Chaudhry & 40 & $\begin{array}{l}19 \\
(47 \%)\end{array}$ & - & - & $0(0.00 \%)$ & - & - & $\begin{array}{l}19 \\
(100.00 \%)\end{array}$ & $\begin{array}{l}21 \\
(52 \%)\end{array}$ & - & - & $6(2$ \\
\hline Giannouchos & 89,756 & $\begin{array}{l}58485 \\
(65 \%)\end{array}$ & $\begin{array}{l}4679 \\
(8.00 \%)\end{array}$ & - & - & - & 53806 (92.00\%) & - & $\begin{array}{l}31271 \\
(34 \%)\end{array}$ & $\begin{array}{l}2721 \\
(8.70 \%)\end{array}$ & - & - \\
\hline $\begin{array}{l}\text { Wang, } \\
\text { Oekelen }\end{array}$ & 57 & $\begin{array}{l}22 \\
(38 \%)\end{array}$ & - & - & $6(27.27 \%)$ & - & - & $\begin{array}{l}16 \\
(72.73 \%)\end{array}$ & $\begin{array}{l}36 \\
(63 \%)\end{array}$ & & - & 15( \\
\hline Miyara & 470 & $\begin{array}{l}132 \\
(28 \%)\end{array}$ & $\begin{array}{l}14 \\
(10.61 \%)\end{array}$ & $\begin{array}{l}41 \\
(31.06 \%)\end{array}$ & & $\begin{array}{l}77 \\
(58.33 \%)\end{array}$ & - & - & $\begin{array}{l}338 \\
(71 \%)\end{array}$ & $\begin{array}{l}18 \\
(5.33 \%)\end{array}$ & $\begin{array}{l}111 \\
(32.84 \%)\end{array}$ & - \\
\hline Suleyman & 463 & $\begin{array}{l}108 \\
(23 \%)\end{array}$ & - & - & $23(21.30 \%)$ & - & - & $\begin{array}{l}85 \\
(78.70 \%)\end{array}$ & $\begin{array}{l}355 \\
(76 \%)\end{array}$ & - & - & 137 \\
\hline Garassino & 196 & $\begin{array}{l}48 \\
(24 \%)\end{array}$ & $\begin{array}{l}10 \\
(20.83 \%)\end{array}$ & $\begin{array}{l}27 \\
\text { (56.25\%) }\end{array}$ & - & $\begin{array}{l}11 \\
(22.92 \%)\end{array}$ & - & - & $\begin{array}{l}152 \\
(77 \%)\end{array}$ & $\begin{array}{l}38 \\
(25.00 \%)\end{array}$ & $\begin{array}{l}84 \\
(55.26 \%)\end{array}$ & - \\
\hline Siso-Almirall & 260 & $\begin{array}{l}119 \\
(45 \%)\end{array}$ & - & - & $31(26.05 \%)$ & - & - & $\begin{array}{l}88 \\
(73.95 \%)\end{array}$ & $\begin{array}{l}141 \\
(54 \%)\end{array}$ & - & - & 50( \\
\hline Gu & 884 & $\begin{array}{l}511 \\
(57 \%)\end{array}$ & $\begin{array}{l}30 \\
(5.87 \%)\end{array}$ & $\begin{array}{l}126 \\
(24.66 \%)\end{array}$ & - & $\begin{array}{l}355 \\
(69.47 \%)\end{array}$ & - & - & $\begin{array}{l}373 \\
(42 \%)\end{array}$ & $\begin{array}{l}10 \\
(2.68 \%)\end{array}$ & $\begin{array}{l}138 \\
(37.00 \%)\end{array}$ & - \\
\hline Killerby & 531 & $\begin{array}{l}311 \\
(58 \%)\end{array}$ & - & - & $37(11.90 \%)$ & $\begin{array}{l}222 \\
(71.38 \%)\end{array}$ & - & $\begin{array}{l}52 \\
(16.72 \%)\end{array}$ & $\begin{array}{l}220 \\
(41 \%)\end{array}$ & - & - & 54( \\
\hline Nguyen & 689 & $\begin{array}{l}333 \\
(48 \%)\end{array}$ & - & - & 57 (17.12\%) & - & - & $\begin{array}{l}276 \\
(82.88 \%)\end{array}$ & $\begin{array}{l}356 \\
(51 \%)\end{array}$ & - & - & 114 \\
\hline Mendv & 689 & 473 & - & - & $84(17.76 \%)$ & - & - & 389 & 216 & & - & 861 \\
\hline
\end{tabular}




\begin{tabular}{|c|c|c|c|c|c|c|c|c|c|c|c|c|}
\hline$\cdots \cdots \cdots$, & $\ldots$ & $(68 \%)$ & & & $\cdots, \ldots \ldots, \ldots$, & & & $(82.24 \%)$ & $(31 \%)$ & & & $\cdots$ \\
\hline Soares & 10,713 & $\begin{array}{l}9561 \\
(89 \%)\end{array}$ & $\begin{array}{l}132 \\
(1.38 \%)\end{array}$ & - & - & - & $9429(98.62 \%)$ & - & $\begin{array}{l}1152 \\
(10 \%)\end{array}$ & $\begin{array}{l}77 \\
(6.68 \%)\end{array}$ & - & - \\
\hline Zobairy & 203 & $\begin{array}{l}65 \\
(32 \%)\end{array}$ & $\begin{array}{l}1 \\
(1.54 \%)\end{array}$ & - & - & - & $64(98.46 \%)$ & - & $\begin{array}{l}138 \\
(67 \%)\end{array}$ & $\begin{array}{l}11 \\
(7.97 \%)\end{array}$ & - & - \\
\hline Izquierdo & 1,006 & $\begin{array}{l}743 \\
(73 \%)\end{array}$ & $\begin{array}{l}52 \\
(7.00 \%)\end{array}$ & - & - & - & $691(93.00 \%)$ & - & $\begin{array}{l}263 \\
(26 \%)\end{array}$ & $\begin{array}{l}16 \\
(6.08 \%)\end{array}$ & - & - \\
\hline Rizzo & 76,819 & $\begin{array}{l}60039 \\
(78 \%)\end{array}$ & $\begin{array}{l}3931 \\
(6.55 \%)\end{array}$ & $\begin{array}{l}11379 \\
(18.95 \%)\end{array}$ & - & $\begin{array}{l}30042 \\
(50.04 \%)\end{array}$ & - & $\begin{array}{l}14687 \\
(24.46 \%)\end{array}$ & $\begin{array}{l}16780 \\
(21 \%)\end{array}$ & $\begin{array}{l}1254 \\
(7.47 \%)\end{array}$ & $\begin{array}{l}4585 \\
(27.32 \%)\end{array}$ & - \\
\hline Pan & 12,084 & $\begin{array}{l}8548 \\
(70 \%)\end{array}$ & - & - & $1263(14.78 \%)$ & - & - & $\begin{array}{l}7285 \\
(85.22 \%)\end{array}$ & $\begin{array}{l}3536 \\
(29 \%)\end{array}$ & - & - & 874 \\
\hline Petrilli & 5,279 & $\begin{array}{l}2538 \\
(48 \%)\end{array}$ & $\begin{array}{l}147 \\
(5.79 \%)\end{array}$ & $\begin{array}{l}337 \\
(13.28 \%)\end{array}$ & - & $\begin{array}{l}1678 \\
(66.12 \%)\end{array}$ & - & $\begin{array}{l}376 \\
(14.81 \%)\end{array}$ & $\begin{array}{l}2741 \\
(51 \%)\end{array}$ & $\begin{array}{l}141 \\
(5.14 \%)\end{array}$ & $\begin{array}{l}565 \\
(20.61 \%)\end{array}$ & - \\
\hline Vilar-Garcia & 328,892 & $\begin{array}{l}291254 \\
(88 \%)\end{array}$ & $\begin{array}{l}64792 \\
(22.25 \%)\end{array}$ & - & - & - & - & $\begin{array}{l}226462 \\
(77.75 \%)\end{array}$ & $\begin{array}{l}37638 \\
(11 \%)\end{array}$ & $\begin{array}{l}9526 \\
(25.31 \%)\end{array}$ & - & - \\
\hline Ibarra-Nava & 416,546 & $\begin{array}{l}302693 \\
(72 \%)\end{array}$ & $\begin{array}{l}26773 \\
(8.84 \%)\end{array}$ & - & - & - & - & $\begin{array}{l}275920 \\
(91.16 \%)\end{array}$ & $\begin{array}{l}113853 \\
(27 \%)\end{array}$ & $\begin{array}{l}8875 \\
(7.80 \%)\end{array}$ & - & - \\
\hline Dashti & 12,347 & $\begin{array}{l}8946 \\
(72 \%)\end{array}$ & $\begin{array}{l}353 \\
(3.95 \%)\end{array}$ & $\begin{array}{l}1099 \\
(12.28 \%)\end{array}$ & - & $\begin{array}{l}5133 \\
(57.38 \%)\end{array}$ & - & $\begin{array}{l}2361 \\
(26.39 \%)\end{array}$ & $\begin{array}{l}3401 \\
(27 \%)\end{array}$ & $\begin{array}{l}210 \\
(6.17 \%)\end{array}$ & $\begin{array}{l}860 \\
(25.29 \%)\end{array}$ & - \\
\hline da Silva Neto & 91 & $\begin{array}{l}44 \\
(48 \%)\end{array}$ & - & - & $4(9.09 \%)$ & - & $40(90.91 \%)$ & - & $\begin{array}{l}47 \\
(51 \%)\end{array}$ & - & - & 14( \\
\hline $\begin{array}{l}\text { Israel, } \\
\text { Schaffer }\end{array}$ & 26,676 & $\begin{array}{l}13706 \\
(51 \%)\end{array}$ & $\begin{array}{l}944 \\
(6.89 \%)\end{array}$ & $\begin{array}{l}2166 \\
(15.80 \%)\end{array}$ & - & $\begin{array}{l}10596 \\
(77.31 \%)\end{array}$ & - & - & $\begin{array}{l}12970 \\
(48 \%)\end{array}$ & $\begin{array}{l}880 \\
(6.78 \%)\end{array}$ & $\begin{array}{l}1936 \\
(14.93 \%)\end{array}$ & - \\
\hline loannou & 10,131 & $\begin{array}{l}6624 \\
(65 \%)\end{array}$ & $\begin{array}{l}716 \\
(10.81 \%)\end{array}$ & $\begin{array}{l}2484 \\
(37.50 \%)\end{array}$ & - & $\begin{array}{l}2542 \\
(38.38 \%)\end{array}$ & - & $\begin{array}{l}882 \\
(13.32 \%)\end{array}$ & $\begin{array}{l}3507 \\
(34 \%)\end{array}$ & $\begin{array}{l}419 \\
(11.95 \%)\end{array}$ & $\begin{array}{l}1593 \\
(45.42 \%)\end{array}$ & - \\
\hline Zhang, Li & 1,596 & $\begin{array}{l}576 \\
(36 \%)\end{array}$ & $\begin{array}{l}63 \\
(10.94 \%)\end{array}$ & $\begin{array}{l}190 \\
(32.99 \%)\end{array}$ & - & $\begin{array}{l}318 \\
(55.21 \%)\end{array}$ & - & $5(0.87 \%)$ & $\begin{array}{l}1020 \\
(63 \%)\end{array}$ & $\begin{array}{l}116 \\
(11.37 \%)\end{array}$ & $\begin{array}{l}429 \\
(42.06 \%)\end{array}$ & - \\
\hline $\begin{array}{l}\text { Parra- } \\
\text { Bracamonte }\end{array}$ & 331,298 & $\begin{array}{l}235840 \\
(71 \%)\end{array}$ & - & - & $16676(7.07 \%)$ & - & - & $\begin{array}{l}219164 \\
(92.93 \%)\end{array}$ & $\begin{array}{l}95458 \\
(28 \%)\end{array}$ & - & - & 751 \\
\hline Jehi & 4,536 & $\begin{array}{l}3578 \\
(78 \%)\end{array}$ & $\begin{array}{l}247 \\
(6.90 \%)\end{array}$ & $\begin{array}{l}943 \\
(26.36 \%)\end{array}$ & - & $\begin{array}{l}1795 \\
(50.17 \%)\end{array}$ & - & $\begin{array}{l}593 \\
(16.57 \%)\end{array}$ & $\begin{array}{l}958 \\
(21 \%)\end{array}$ & $\begin{array}{l}82 \\
(8.56 \%)\end{array}$ & $\begin{array}{l}349 \\
(36.43 \%)\end{array}$ & - \\
\hline Arleo & 70 & $\begin{array}{l}36 \\
(51 \%)\end{array}$ & $\begin{array}{l}1 \\
(2.78 \%)\end{array}$ & $\begin{array}{l}10 \\
(27.78 \%)\end{array}$ & - & $\begin{array}{l}25 \\
(69.44 \%)\end{array}$ & - & - & $\begin{array}{l}34 \\
(48 \%)\end{array}$ & $\begin{array}{l}0 \\
(0.00 \%)\end{array}$ & $\begin{array}{l}10 \\
(29.41 \%)\end{array}$ & - \\
\hline Kortela & 604 & $\begin{array}{l}246 \\
(40 \%)\end{array}$ & $\begin{array}{l}12 \\
(4.88 \%)\end{array}$ & $\begin{array}{l}14 \\
(5.69 \%)\end{array}$ & - & $\begin{array}{l}55 \\
(22.36 \%)\end{array}$ & - & $\begin{array}{l}165 \\
(67.07 \%)\end{array}$ & $\begin{array}{l}328 \\
(54 \%)\end{array}$ & $\begin{array}{l}14 \\
(4.27 \%)\end{array}$ & $\begin{array}{l}66 \\
(20.12 \%)\end{array}$ & - \\
\hline Didikoglu & 7,733 & $\begin{array}{l}5239 \\
(67 \%)\end{array}$ & $\begin{array}{l}554 \\
(10.57 \%)\end{array}$ & $\begin{array}{l}1845 \\
(35.22 \%)\end{array}$ & - & $\begin{array}{l}2841 \\
(54.23 \%)\end{array}$ & - & - & $\begin{array}{l}2494 \\
(32 \%)\end{array}$ & $\begin{array}{l}313 \\
(12.55 \%)\end{array}$ & $\begin{array}{l}1121 \\
(44.95 \%)\end{array}$ & - \\
\hline $\begin{array}{l}\text { Ho, } \\
\text { Narasimhan }\end{array}$ & 9,991 & $\begin{array}{l}5082 \\
(50 \%)\end{array}$ & $\begin{array}{l}189 \\
(3.72 \%)\end{array}$ & $\begin{array}{l}744 \\
(14.64 \%)\end{array}$ & - & $\begin{array}{l}4148 \\
(81.62 \%)\end{array}$ & - & - & $\begin{array}{l}4909 \\
(49 \%)\end{array}$ & $\begin{array}{l}217 \\
(4.42 \%)\end{array}$ & $\begin{array}{l}1062 \\
(21.63 \%)\end{array}$ & - \\
\hline Saurabh & 911 & $\begin{array}{l}69 \\
(7 \%)\end{array}$ & $\begin{array}{l}6 \\
(8.70 \%)\end{array}$ & $\begin{array}{l}0 \\
(0.00 \%)\end{array}$ & - & $\begin{array}{l}63 \\
(91.30 \%)\end{array}$ & - & - & $\begin{array}{l}842 \\
(92 \%)\end{array}$ & $\begin{array}{l}68 \\
(8.08 \%)\end{array}$ & $\begin{array}{l}6 \\
(0.71 \%)\end{array}$ & - \\
\hline Munoz & 314 & $\begin{array}{l}294 \\
(93 \%)\end{array}$ & $\begin{array}{l}20 \\
(6.80 \%)\end{array}$ & - & - & - & - & $\begin{array}{l}274 \\
(93.20 \%)\end{array}$ & $\begin{array}{l}20 \\
(6 \%)\end{array}$ & $\begin{array}{l}1 \\
(5.00 \%)\end{array}$ & - & - \\
\hline Cummins & 1,781 & $\begin{array}{l}586 \\
(32 \%)\end{array}$ & $\begin{array}{l}77 \\
(13.14 \%)\end{array}$ & - & - & - & - & $\begin{array}{l}509 \\
(86.86 \%)\end{array}$ & $\begin{array}{l}1195 \\
(67 \%)\end{array}$ & $\begin{array}{l}104 \\
(8.70 \%)\end{array}$ & - & - \\
\hline 4 & & & & & & & & & & & & - \\
\hline
\end{tabular}


Forest plot of current smokers and risk of hospital admission

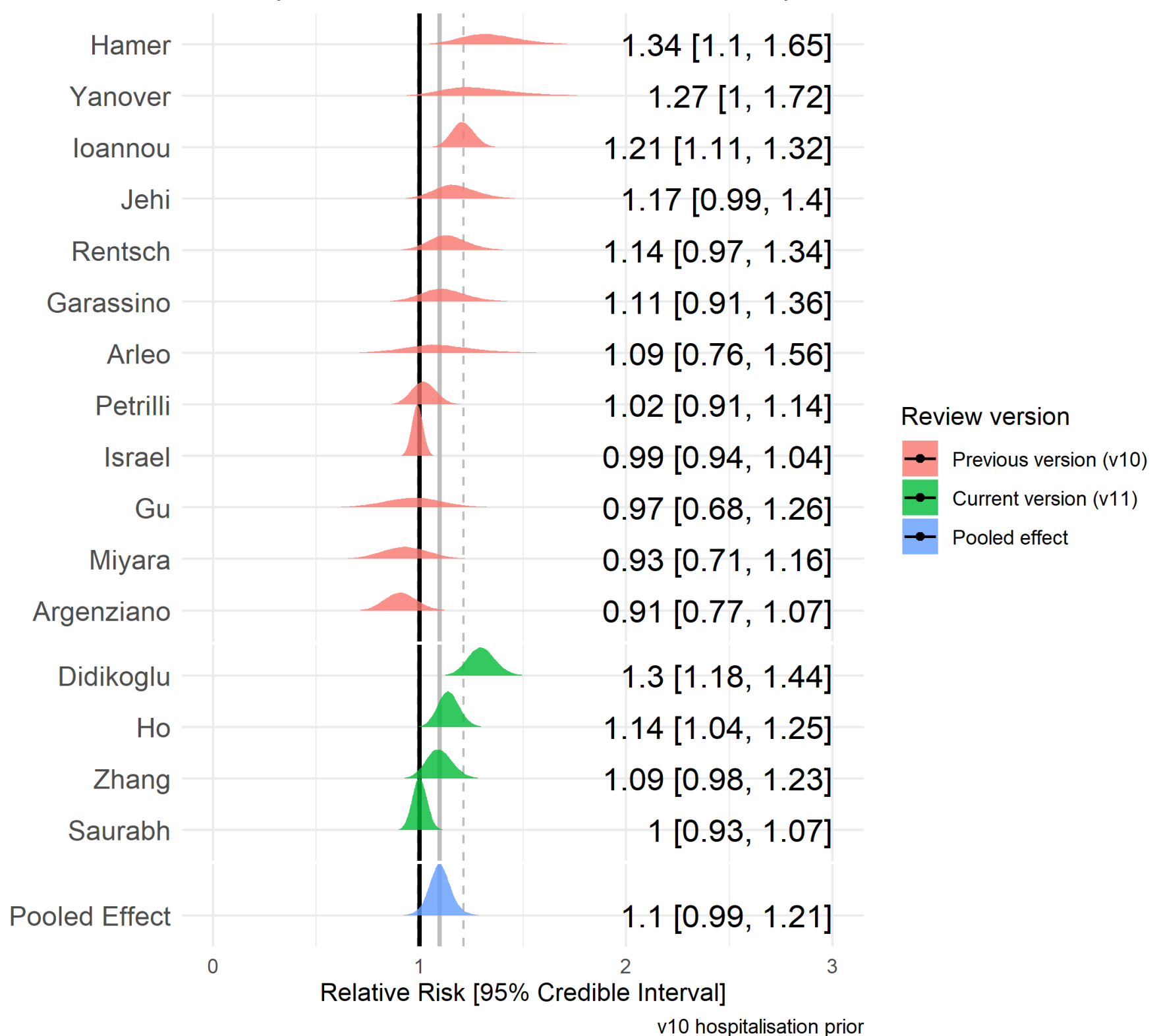

Figure 5. Forest plot for risk of hospitalisation in current vs. never smokers. The prior from the previous review version (v10) was $R R=1.08$. 


\section{Forest plot of former smokers and risk of hospital admission}

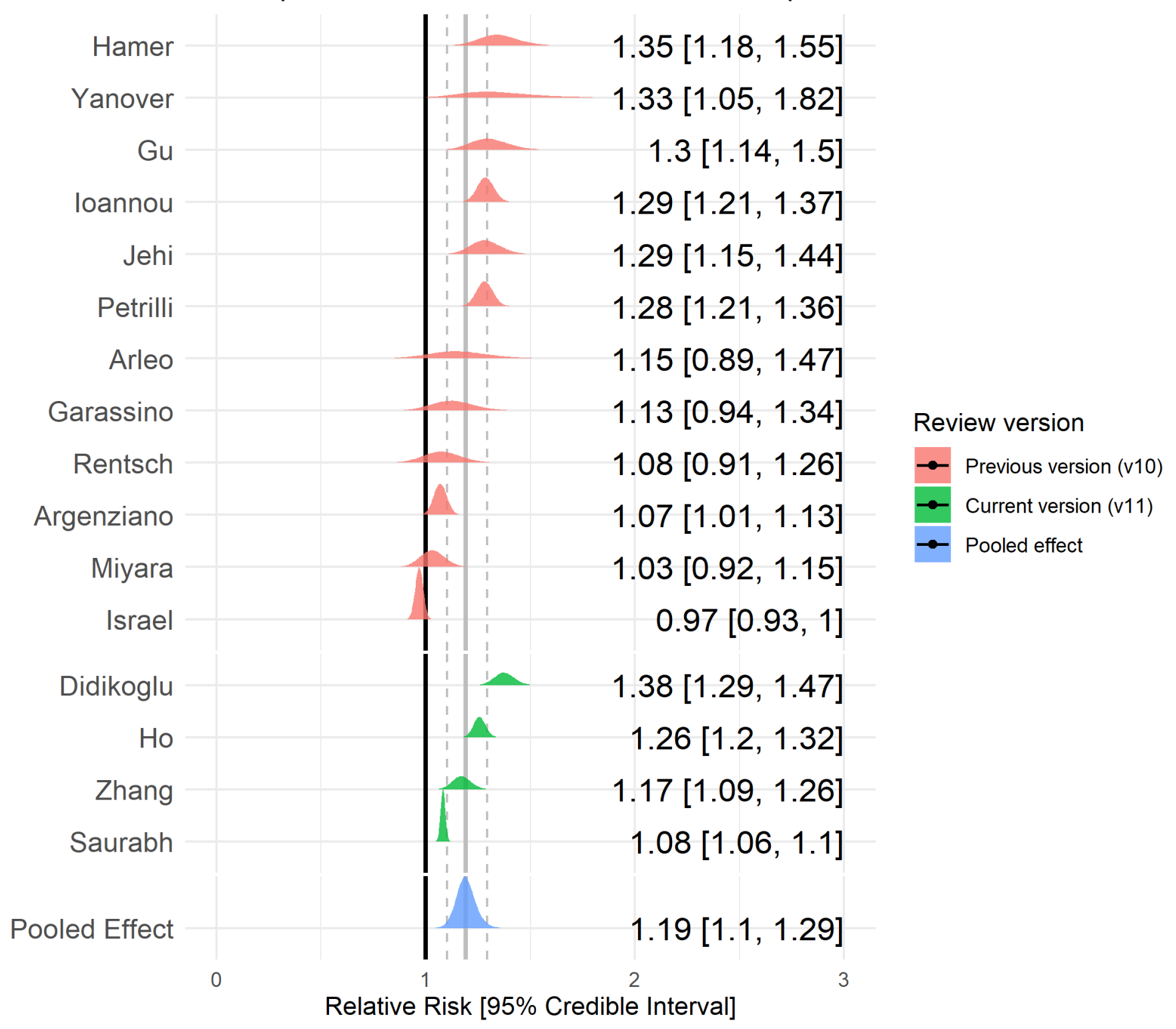

v10 hospitalisation prior

Figure 6. Forest plot for risk of hospitalisation in former vs. never smokers. The prior from the previous version (v10) was $R R=1.18$.

Disease severity by smoking status

85 studies reported disease severity in hospitalised patients stratified by smoking status (see Table 4). Severe (as opposed to non-severe) disease was broadly defined as requiring intensive treatment unit (ITU) admission, requiring oxygen as a hospital inpatient or in-hospital death. Meta-analyses were performed for 16 'fair' quality studies (see Figure 7 and 8). Current $(R R=1.26,95 \% \mathrm{Crl}=0.92-1.73, \tau=0.32)$ and former $(\mathrm{R} R=1.8,95 \% \mathrm{Crl}=1.27-2.55, \tau=0.46) \mathrm{compared}$ with never smokers were at increased risk of greater disease severity. However, while data for current smokers only were inconclusive, they favoured there being a small but important association. The probability of current and former smokers having increased risk of greater disease severity $(R R \geq 1.1)$ compared with never smokers was $80 \%$ and $100 \%$, 
respectively. Results were materially unchanged in two sensitivity analyses.

Table 4 COVID-19 disease severity by smoking status

\begin{tabular}{|c|c|c|c|c|c|c|c|c|c|c|c|c|}
\hline \multirow[b]{2}{*}{ Author } & \multirow[b]{2}{*}{$\begin{array}{l}\text { Population } \\
\text { with } \\
\text { severity }\end{array}$} & \multicolumn{7}{|c|}{ Non severe disease } & \multicolumn{4}{|c|}{ Severe disease } \\
\hline & & $\mathbf{N}(\%)$ & $\begin{array}{l}\text { Current } \\
\text { smoker } \\
(\%)\end{array}$ & $\begin{array}{l}\text { Former } \\
\text { smoker } \\
(\%)\end{array}$ & $\begin{array}{l}\text { Current/former } \\
\text { smoker (\%) }\end{array}$ & $\begin{array}{l}\text { Never } \\
\text { smoker } \\
(\%)\end{array}$ & $\begin{array}{l}\text { Never/unknown } \\
\text { smoker (\%) }\end{array}$ & $\begin{array}{l}\text { Not } \\
\text { stated } \\
(\%)\end{array}$ & $\mathrm{N}(\%)$ & $\begin{array}{l}\text { Current } \\
\text { smoker } \\
(\%)\end{array}$ & $\begin{array}{l}\text { Former } \\
\text { smoker } \\
(\%)\end{array}$ & $\begin{array}{l}\text { C } \\
\text { si }\end{array}$ \\
\hline Guan, Ni & 1,085 & $\begin{array}{l}913 \\
(84 \%)\end{array}$ & $\begin{array}{l}108 \\
(11.83 \%)\end{array}$ & $\begin{array}{l}12 \\
(1.31 \%)\end{array}$ & - & $\begin{array}{l}793 \\
(86.86 \%)\end{array}$ & - & - & $\begin{array}{l}172 \\
(15 \%)\end{array}$ & $\begin{array}{l}29 \\
(16.86 \%)\end{array}$ & $\begin{array}{l}9 \\
(5.23 \%)\end{array}$ & - \\
\hline Zhang, Dong & 9 & $\begin{array}{l}3 \\
(33 \%)\end{array}$ & $0(0.00 \%)$ & $\begin{array}{l}3 \\
(100.00 \%)\end{array}$ & - & $\begin{array}{l}0 \\
(0.00 \%)\end{array}$ & - & - & $\begin{array}{l}6 \\
(66 \%)\end{array}$ & $\begin{array}{l}2 \\
(33.33 \%)\end{array}$ & $\begin{array}{l}4 \\
(66.67 \%)\end{array}$ & - \\
\hline Wan & 9 & $\begin{array}{l}8 \\
(88 \%)\end{array}$ & $\begin{array}{l}8 \\
(100.00 \%)\end{array}$ & $0(0.00 \%)$ & - & $\begin{array}{l}0 \\
(0.00 \%)\end{array}$ & - & - & $\begin{array}{l}1 \\
(11 \%)\end{array}$ & $\begin{array}{l}1 \\
(100.00 \%)\end{array}$ & $\begin{array}{l}0 \\
(0.00 \%)\end{array}$ & - \\
\hline $\begin{array}{l}\text { Huang, } \\
\text { Wang }\end{array}$ & 3 & $\begin{array}{l}3 \\
(100 \%)\end{array}$ & $\begin{array}{l}3 \\
(100.00 \%)\end{array}$ & $0(0.00 \%)$ & - & $\begin{array}{l}0 \\
(0.00 \%)\end{array}$ & - & - & $0(0 \%)$ & 0 (NaN\%) & $\begin{array}{l}0 \\
\text { (NaN\%) }\end{array}$ & - \\
\hline Rentsch & 285 & $\begin{array}{l}168 \\
(58 \%)\end{array}$ & $\begin{array}{l}47 \\
(27.98 \%)\end{array}$ & $\begin{array}{l}53 \\
(31.55 \%)\end{array}$ & - & $\begin{array}{l}68 \\
(40.48 \%)\end{array}$ & - & - & $\begin{array}{l}117 \\
(41 \%)\end{array}$ & $\begin{array}{l}43 \\
(36.75 \%)\end{array}$ & $\begin{array}{l}36 \\
(30.77 \%)\end{array}$ & - \\
\hline $\mathrm{Hu}$ & 323 & $\begin{array}{l}151 \\
(46 \%)\end{array}$ & - & - & 12 (7.95\%) & - & 139 (92.05\%) & - & $\begin{array}{l}172 \\
(53 \%)\end{array}$ & - & - & $2 t$ \\
\hline Wang, Pan & 125 & $\begin{array}{l}100 \\
(80 \%)\end{array}$ & - & - & $9(9.00 \%)$ & - & $91(91.00 \%)$ & - & $\begin{array}{l}25 \\
(20 \%)\end{array}$ & - & - & 7 \\
\hline Kim & 27 & $\begin{array}{l}21 \\
(77 \%)\end{array}$ & $\begin{array}{l}3 \\
(14.29 \%)\end{array}$ & - & - & - & $18(85.71 \%)$ & - & $\begin{array}{l}6 \\
(22 \%)\end{array}$ & $\begin{array}{l}2 \\
(33.33 \%)\end{array}$ & $\begin{array}{l}0 \\
(0.00 \%)\end{array}$ & - \\
\hline Shi, Yu & 474 & $\begin{array}{l}425 \\
(89 \%)\end{array}$ & - & - & $34(8.00 \%)$ & & 391 (92.00\%) & - & $\begin{array}{l}49 \\
(10 \%)\end{array}$ & - & - & 6 \\
\hline Liao, Feng & 148 & $\begin{array}{l}92 \\
(62 \%)\end{array}$ & - & - & $5(5.43 \%)$ & - & - & $\begin{array}{l}87 \\
(94.57 \%)\end{array}$ & $\begin{array}{l}56 \\
(37 \%)\end{array}$ & $3(5.36 \%)$ & - & - \\
\hline Shi, Ren & 134 & $\begin{array}{l}88 \\
(65 \%)\end{array}$ & - & - & $8(9.09 \%)$ & - & - & $\begin{array}{l}80 \\
(90.91 \%)\end{array}$ & $\begin{array}{l}46 \\
(34 \%)\end{array}$ & - & - & 6 \\
\hline Hadjadj & 50 & $\begin{array}{l}15 \\
(30 \%)\end{array}$ & $1(6.67 \%)$ & $\begin{array}{l}2 \\
(13.33 \%)\end{array}$ & - & $\begin{array}{l}12 \\
(80.00 \%)\end{array}$ & - & - & $\begin{array}{l}35 \\
(70 \%)\end{array}$ & $0(0.00 \%)$ & $\begin{array}{l}7 \\
(20.00 \%)\end{array}$ & - \\
\hline Zheng, Xiong & 73 & $\begin{array}{l}43 \\
(58 \%)\end{array}$ & - & - & $6(13.95 \%)$ & $\begin{array}{l}37 \\
(86.05 \%)\end{array}$ & - & - & $\begin{array}{l}30 \\
(41 \%)\end{array}$ & - & - & 2 \\
\hline de la Rica & 48 & $\begin{array}{l}26 \\
(54 \%)\end{array}$ & - & - & $6(23.08 \%)$ & - & - & $\begin{array}{l}20 \\
(76.92 \%)\end{array}$ & $\begin{array}{l}20 \\
(41 \%)\end{array}$ & - & - & 4 \\
\hline Yin, Yang & 106 & $\begin{array}{l}47 \\
(44 \%)\end{array}$ & - & - & $6(12.77 \%)$ & - & - & $\begin{array}{l}41 \\
(87.23 \%)\end{array}$ & $\begin{array}{l}59 \\
(55 \%)\end{array}$ & - & - & $11^{\prime}$ \\
\hline Allenbach & 147 & $\begin{array}{l}100 \\
(68 \%)\end{array}$ & - & - & $9(9.00 \%)$ & - & - & $\begin{array}{l}91 \\
(91.00 \%)\end{array}$ & $\begin{array}{l}47 \\
(31 \%)\end{array}$ & - & - & 0 \\
\hline Goyal & 393 & $\begin{array}{l}263 \\
(66 \%)\end{array}$ & $\begin{array}{l}14 \\
(5.32 \%)\end{array}$ & - & - & - & - & $\begin{array}{l}249 \\
(94.68 \%)\end{array}$ & $\begin{array}{l}130 \\
(33 \%)\end{array}$ & $6(4.62 \%)$ & - & - \\
\hline Feng & 454 & $\begin{array}{l}333 \\
(73 \%)\end{array}$ & $\begin{array}{l}27 \\
(8.11 \%)\end{array}$ & - & - & - & - & $\begin{array}{l}306 \\
(91.89 \%)\end{array}$ & $\begin{array}{l}121 \\
(26 \%)\end{array}$ & $\begin{array}{l}17 \\
(14.05 \%)\end{array}$ & - & - \\
\hline Yao & 108 & $\begin{array}{l}83 \\
(76 \%)\end{array}$ & $1(1.20 \%)$ & - & - & - & - & $\begin{array}{l}82 \\
(98.80 \%)\end{array}$ & $\begin{array}{l}25 \\
(23 \%)\end{array}$ & $\begin{array}{l}3 \\
(12.00 \%)\end{array}$ & - & - \\
\hline Regina & 200 & $\begin{array}{l}163 \\
(81 \%)\end{array}$ & $9(5.52 \%)$ & - & - & - & - & $\begin{array}{l}154 \\
(94.48 \%)\end{array}$ & $\begin{array}{l}37 \\
(18 \%)\end{array}$ & $0(0.00 \%)$ & - & - \\
\hline Feuth & 28 & $\begin{array}{l}21 \\
(75 \%)\end{array}$ & $1(4.76 \%)$ & $\begin{array}{l}7 \\
\text { (33.33\%) }\end{array}$ & - & $\begin{array}{l}13 \\
(61.90 \%)\end{array}$ & - & - & $\begin{array}{l}7 \\
(25 \%)\end{array}$ & $\begin{array}{l}2 \\
\text { (28.57\%) }\end{array}$ & $\begin{array}{l}1 \\
(14.29 \%)\end{array}$ & \\
\hline
\end{tabular}




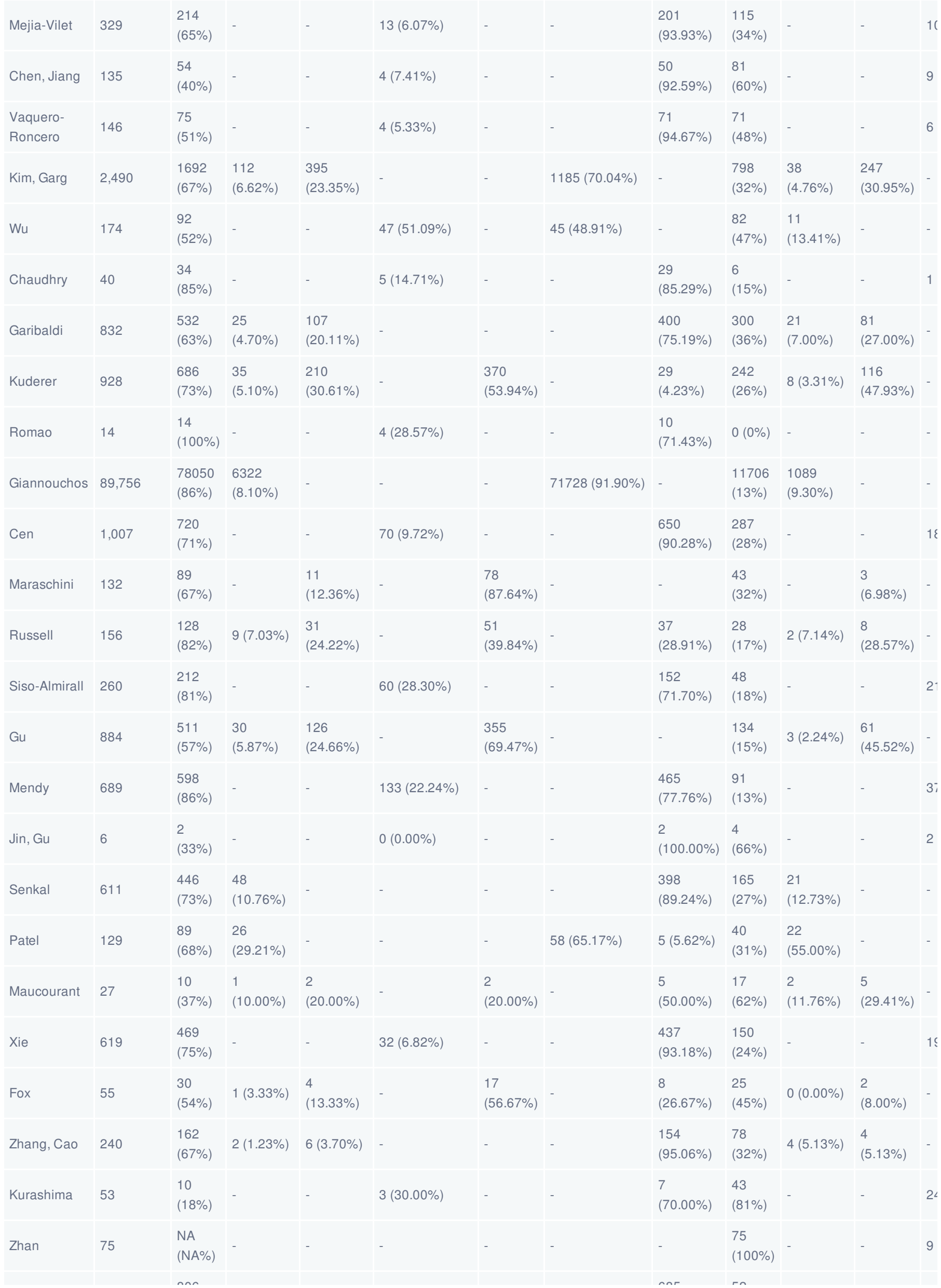




\begin{tabular}{|c|c|c|c|c|c|c|c|c|c|c|c|c|}
\hline Omrani & 858 & $\begin{array}{l}\text { ৪Ub } \\
(93 \%)\end{array}$ & - & - & $121(15.01 \%)$ & - & - & $\begin{array}{l}\text { ь४১ } \\
\text { (84.99\%) }\end{array}$ & $\begin{array}{l}3< \\
(6 \%)\end{array}$ & - & - & 9 \\
\hline Marcos & 918 & $\begin{array}{l}555 \\
(60 \%)\end{array}$ & $\begin{array}{l}38 \\
(6.85 \%)\end{array}$ & - & $69(12.43 \%)$ & - & - & $\begin{array}{l}448 \\
(80.72 \%)\end{array}$ & $\begin{array}{l}363 \\
(39 \%)\end{array}$ & $\begin{array}{l}18 \\
(4.96 \%)\end{array}$ & - & $7^{\circ}$ \\
\hline $\begin{array}{l}\text { Hoertel, } \\
\text { Sanchez, } \\
\text { Rico }\end{array}$ & 7,345 & $\begin{array}{l}6014 \\
(81 \%)\end{array}$ & $\begin{array}{l}433 \\
(7.20 \%)\end{array}$ & - & - & - & - & $\begin{array}{l}5581 \\
(92.80 \%)\end{array}$ & $\begin{array}{l}1331 \\
(18 \%)\end{array}$ & $\begin{array}{l}190 \\
(14.27 \%)\end{array}$ & - & - \\
\hline Qi & 267 & $\begin{array}{l}217 \\
(81 \%)\end{array}$ & $\begin{array}{l}22 \\
(10.14 \%)\end{array}$ & - & - & - & 195 (89.86\%) & - & $\begin{array}{l}50 \\
(18 \%)\end{array}$ & $\begin{array}{l}31 \\
(62.00 \%)\end{array}$ & - & - \\
\hline Monteiro & 112 & $\begin{array}{l}84 \\
(75 \%)\end{array}$ & $3(3.57 \%)$ & $\begin{array}{l}14 \\
(16.67 \%)\end{array}$ & - & $\begin{array}{l}63 \\
(75.00 \%)\end{array}$ & - & $4(4.76 \%)$ & $\begin{array}{l}28 \\
(25 \%)\end{array}$ & $\begin{array}{l}4 \\
(14.29 \%)\end{array}$ & $\begin{array}{l}6 \\
(21.43 \%)\end{array}$ & - \\
\hline Morshed & 103 & $\begin{array}{l}87 \\
(84 \%)\end{array}$ & $\begin{array}{l}28 \\
(32.18 \%)\end{array}$ & - & - & - & $59(67.82 \%)$ & - & $\begin{array}{l}16 \\
(15 \%)\end{array}$ & $\begin{array}{l}4 \\
(25.00 \%)\end{array}$ & - & - \\
\hline Zhou, Sun & 144 & $\begin{array}{l}108 \\
(75 \%)\end{array}$ & $\begin{array}{l}11 \\
(10.19 \%)\end{array}$ & - & - & - & - & $\begin{array}{l}97 \\
(89.81 \%)\end{array}$ & $\begin{array}{l}36 \\
(25 \%)\end{array}$ & $2(5.56 \%)$ & - & - \\
\hline $\begin{array}{l}\text { Hippisley- } \\
\text { Cox }\end{array}$ & - & $\begin{array}{l}\text { NA } \\
\text { (NA\%) }\end{array}$ & - & - & - & - & - & - & $\begin{array}{l}1286 \\
\text { (NA\%) }\end{array}$ & $\begin{array}{l}56 \\
(4.35 \%)\end{array}$ & $\begin{array}{l}427 \\
(33.20 \%)\end{array}$ & - \\
\hline Zhao, Chen & 641 & $\begin{array}{l}398 \\
(62 \%)\end{array}$ & $\begin{array}{l}87 \\
(21.86 \%)\end{array}$ & - & - & - & - & $\begin{array}{l}311 \\
(78.14 \%)\end{array}$ & $\begin{array}{l}195 \\
(30 \%)\end{array}$ & $\begin{array}{l}52 \\
(26.67 \%)\end{array}$ & - & - \\
\hline Qu & 246 & $\begin{array}{l}226 \\
(91 \%)\end{array}$ & $\begin{array}{l}90 \\
(39.82 \%)\end{array}$ & - & - & - & - & $\begin{array}{l}136 \\
(60.18 \%)\end{array}$ & $\begin{array}{l}20 \\
(8 \%)\end{array}$ & $\begin{array}{l}14 \\
(70.00 \%)\end{array}$ & - & - \\
\hline Petrilli & 2,729 & $\begin{array}{l}1739 \\
(63 \%)\end{array}$ & $\begin{array}{l}97 \\
(5.58 \%)\end{array}$ & $\begin{array}{l}325 \\
(18.69 \%)\end{array}$ & - & $\begin{array}{l}1067 \\
(61.36 \%)\end{array}$ & - & $\begin{array}{l}250 \\
(14.38 \%)\end{array}$ & $\begin{array}{l}990 \\
(36 \%)\end{array}$ & $\begin{array}{l}44 \\
(4.44 \%)\end{array}$ & $\begin{array}{l}236 \\
(23.84 \%)\end{array}$ & - \\
\hline Ren & 432 & $\begin{array}{l}314 \\
(72 \%)\end{array}$ & $\begin{array}{l}26 \\
(8.28 \%)\end{array}$ & - & - & $\begin{array}{l}288 \\
(91.72 \%)\end{array}$ & - & - & $\begin{array}{l}118 \\
(27 \%)\end{array}$ & $\begin{array}{l}17 \\
(14.41 \%)\end{array}$ & - & - \\
\hline Yan & 578 & $\begin{array}{l}450 \\
(77 \%)\end{array}$ & $\begin{array}{l}31 \\
(6.89 \%)\end{array}$ & - & - & - & - & $\begin{array}{l}419 \\
(93.11 \%)\end{array}$ & $\begin{array}{l}128 \\
(22 \%)\end{array}$ & $\begin{array}{l}20 \\
(15.62 \%)\end{array}$ & - & - \\
\hline Nicholson & 1,042 & $\begin{array}{l}550 \\
(52 \%)\end{array}$ & $\begin{array}{l}37 \\
(6.73 \%)\end{array}$ & $\begin{array}{l}106 \\
(19.27 \%)\end{array}$ & - & $\begin{array}{l}211 \\
(38.36 \%)\end{array}$ & - & $\begin{array}{l}196 \\
(35.64 \%)\end{array}$ & $\begin{array}{l}401 \\
(38 \%)\end{array}$ & $\begin{array}{l}41 \\
(10.22 \%)\end{array}$ & $\begin{array}{l}92 \\
(22.94 \%)\end{array}$ & - \\
\hline Zhu & 432 & $\begin{array}{l}285 \\
(65 \%)\end{array}$ & $\begin{array}{l}46 \\
(16.14 \%)\end{array}$ & - & - & - & - & $\begin{array}{l}239 \\
(83.86 \%)\end{array}$ & $\begin{array}{l}147 \\
(34 \%)\end{array}$ & $\begin{array}{l}16 \\
(10.88 \%)\end{array}$ & - & - \\
\hline Kalan & 193 & $\begin{array}{l}122 \\
(63 \%)\end{array}$ & $9(7.38 \%)$ & - & - & $\begin{array}{l}102 \\
(83.61 \%)\end{array}$ & - & $\begin{array}{l}11 \\
(9.02 \%)\end{array}$ & $\begin{array}{l}71 \\
(36 \%)\end{array}$ & $5(7.04 \%)$ & - & - \\
\hline Burrell & 204 & $\begin{array}{l}85 \\
(41 \%)\end{array}$ & - & - & 7 (8.24\%) & - & 75 (88.24\%) & 3 (3.53\%) & $\begin{array}{l}119 \\
(58 \%)\end{array}$ & - & - & 2( \\
\hline Chudasama & 1,706 & $\begin{array}{l}\text { NA } \\
\text { (NA\%) }\end{array}$ & - & - & - & - & - & - & $\begin{array}{l}1706 \\
(100 \%)\end{array}$ & $\begin{array}{l}235 \\
(13.77 \%)\end{array}$ & $\begin{array}{l}699 \\
(40.97 \%)\end{array}$ & - \\
\hline Lamure & 89 & $\begin{array}{l}\text { NA } \\
\text { (NA\%) }\end{array}$ & - & - & - & - & - & - & $\begin{array}{l}25 \\
(28 \%)\end{array}$ & $1(4.00 \%)$ & $\begin{array}{l}5 \\
(20.00 \%)\end{array}$ & - \\
\hline $\begin{array}{l}\text { Zhou, He, } \\
\text { Yang }\end{array}$ & 1,087 & $\begin{array}{l}990 \\
(91 \%)\end{array}$ & - & - & $849(85.76 \%)$ & $\begin{array}{l}141 \\
(14.24 \%)\end{array}$ & - & - & $\begin{array}{l}97 \\
(8 \%)\end{array}$ & - & - & $7 !$ \\
\hline Zhou, Qin & 51 & $\begin{array}{l}\text { NA } \\
\text { (NA\%) }\end{array}$ & - & - & - & - & - & - & $\begin{array}{l}51 \\
(100 \%)\end{array}$ & - & - & 4( \\
\hline Zhan, Liu & 405 & $\begin{array}{l}257 \\
(63 \%)\end{array}$ & - & - & $21(8.17 \%)$ & $\begin{array}{l}236 \\
(91.83 \%)\end{array}$ & - & - & $\begin{array}{l}148 \\
(36 \%)\end{array}$ & - & - & $2 !$ \\
\hline $\begin{array}{l}\text { Li, Long, } \\
\text { Zhang }\end{array}$ & 954 & $\begin{array}{l}838 \\
(87 \%)\end{array}$ & - & - & $34(4.06 \%)$ & $\begin{array}{l}804 \\
(95.94 \%)\end{array}$ & - & - & $\begin{array}{l}116 \\
(12 \%)\end{array}$ & - & - & $2 ;$ \\
\hline Jakob & 2,155 & $\begin{array}{l}1400 \\
(64 \%)\end{array}$ & - & - & $92(6.57 \%)$ & $\begin{array}{l}99 \\
(7.07 \%)\end{array}$ & - & $\begin{array}{l}669 \\
(47.79 \%)\end{array}$ & $\begin{array}{l}755 \\
(35 \%)\end{array}$ & $\begin{array}{l}51 \\
(6.75 \%)\end{array}$ & $\begin{array}{l}58 \\
(7.68 \%)\end{array}$ & - \\
\hline Aksu & 123 & $\begin{array}{l}34 \\
(27 \%)\end{array}$ & $3(8.82 \%)$ & - & - & - & $31(91.18 \%)$ & - & $\begin{array}{l}89 \\
(72 \%)\end{array}$ & $\begin{array}{l}11 \\
(12.36 \%)\end{array}$ & - & - \\
\hline
\end{tabular}




\begin{tabular}{|c|c|c|c|c|c|c|c|c|c|c|c|c|}
\hline Adrish & 1,173 & $\begin{array}{l}162 \\
(13 \%)\end{array}$ & - & - & $36(22.22 \%)$ & - & $126(77.78 \%)$ & - & $\begin{array}{l}1011 \\
(86 \%)\end{array}$ & - & - & 3( \\
\hline $\begin{array}{l}\text { Hoertel, } \\
\text { Sanchez, } \\
\text { Vernet }\end{array}$ & 12,210 & $\begin{array}{l}11018 \\
(90 \%)\end{array}$ & $\begin{array}{l}921 \\
(8.36 \%)\end{array}$ & - & - & - & - & $\begin{array}{l}10097 \\
(91.64 \%)\end{array}$ & $\begin{array}{l}1192 \\
(9 \%)\end{array}$ & $\begin{array}{l}181 \\
(15.18 \%)\end{array}$ & - & - \\
\hline $\begin{array}{l}\text { Vila- } \\
\text { Corcoles, } \\
\text { Satue-Gracia }\end{array}$ & 282 & $\begin{array}{l}218 \\
(77 \%)\end{array}$ & - & - & 21 (9.63\%) & - & - & $\begin{array}{l}197 \\
(90.37 \%)\end{array}$ & $\begin{array}{l}64 \\
(22 \%)\end{array}$ & - & - & 4 \\
\hline Boyd & 38 & $0(0 \%)$ & - & - & - & - & - & - & $\begin{array}{l}38 \\
(100 \%)\end{array}$ & - & - & 2 \\
\hline Caliskan & 565 & $\begin{array}{l}474 \\
(83 \%)\end{array}$ & $\begin{array}{l}96 \\
(20.25 \%)\end{array}$ & $\begin{array}{l}45 \\
(9.49 \%)\end{array}$ & - & $\begin{array}{l}333 \\
(70.25 \%)\end{array}$ & - & - & $\begin{array}{l}91 \\
(16 \%)\end{array}$ & $\begin{array}{l}22 \\
(24.18 \%)\end{array}$ & $\begin{array}{l}34 \\
(37.36 \%)\end{array}$ & - \\
\hline Ebrahimian & 226 & $\begin{array}{l}134 \\
(59 \%)\end{array}$ & - & - & 12 (8.96\%) & - & 122 (91.04\%) & - & $\begin{array}{l}92 \\
(40 \%)\end{array}$ & - & - & $1 c^{\prime}$ \\
\hline $\begin{array}{l}\text { Ho, } \\
\text { Narasimhan }\end{array}$ & 4,909 & $\begin{array}{l}3859 \\
(78 \%)\end{array}$ & $\begin{array}{l}169 \\
(4.38 \%)\end{array}$ & $\begin{array}{l}833 \\
(21.59 \%)\end{array}$ & - & - & 2857 (74.03\%) & - & $\begin{array}{l}1050 \\
(21 \%)\end{array}$ & $\begin{array}{l}48 \\
(4.57 \%)\end{array}$ & $\begin{array}{l}229 \\
(21.81 \%)\end{array}$ & - \\
\hline Quan & 2,038 & $\begin{array}{l}1452 \\
(71 \%)\end{array}$ & - & $\begin{array}{l}549 \\
(37.81 \%)\end{array}$ & - & - & - & $\begin{array}{l}903 \\
(62.19 \%)\end{array}$ & $\begin{array}{l}586 \\
(28 \%)\end{array}$ & - & - & 26 \\
\hline Saurabh & 911 & $\begin{array}{l}783 \\
(85 \%)\end{array}$ & $\begin{array}{l}65 \\
(8.30 \%)\end{array}$ & $3(0.38 \%)$ & - & $\begin{array}{l}715 \\
(91.32 \%)\end{array}$ & - & - & $\begin{array}{l}128 \\
(14 \%)\end{array}$ & 9 (7.03\%) & $\begin{array}{l}3 \\
(2.34 \%)\end{array}$ & - \\
\hline Chousein & 114 & $\begin{array}{l}94 \\
(82 \%)\end{array}$ & $\begin{array}{l}17 \\
(18.09 \%)\end{array}$ & $\begin{array}{l}17 \\
(18.09 \%)\end{array}$ & - & $\begin{array}{l}60 \\
(63.83 \%)\end{array}$ & - & - & $\begin{array}{l}20 \\
(17 \%)\end{array}$ & $\begin{array}{l}2 \\
(10.00 \%)\end{array}$ & $\begin{array}{l}6 \\
(30.00 \%)\end{array}$ & - \\
\hline Tavakol & 206 & $\begin{array}{l}182 \\
(88 \%)\end{array}$ & - & - & $24(13.19 \%)$ & - & 158 (86.81\%) & - & $\begin{array}{l}24 \\
(11 \%)\end{array}$ & - & - & 2 \\
\hline $\begin{array}{l}\text { Yao, } \\
\text { Hasegawa }\end{array}$ & 101 & $\begin{array}{l}78 \\
(77 \%)\end{array}$ & - & - & 17 (21.79\%) & - & 71 (91.03\%) & - & $\begin{array}{l}23 \\
(22 \%)\end{array}$ & - & - & $1 c^{\prime}$ \\
\hline Cummins & 1,195 & $\begin{array}{l}1043 \\
(87 \%)\end{array}$ & $\begin{array}{l}96 \\
(9.20 \%)\end{array}$ & - & - & - & - & $\begin{array}{l}947 \\
(90.80 \%)\end{array}$ & $\begin{array}{l}152 \\
(12 \%)\end{array}$ & 9 (5.92\%) & - & - \\
\hline Nuno & 4,730 & $\begin{array}{l}3536 \\
(74 \%)\end{array}$ & $\begin{array}{l}333 \\
(9.42 \%)\end{array}$ & - & - & - & - & $\begin{array}{l}3203 \\
(90.58 \%)\end{array}$ & $\begin{array}{l}1194 \\
(25 \%)\end{array}$ & $\begin{array}{l}147 \\
(12.31 \%)\end{array}$ & - & - \\
\hline 4 & & & & & & & & & & & & - \\
\hline
\end{tabular}


Forest plot of current smokers and risk of severe disease

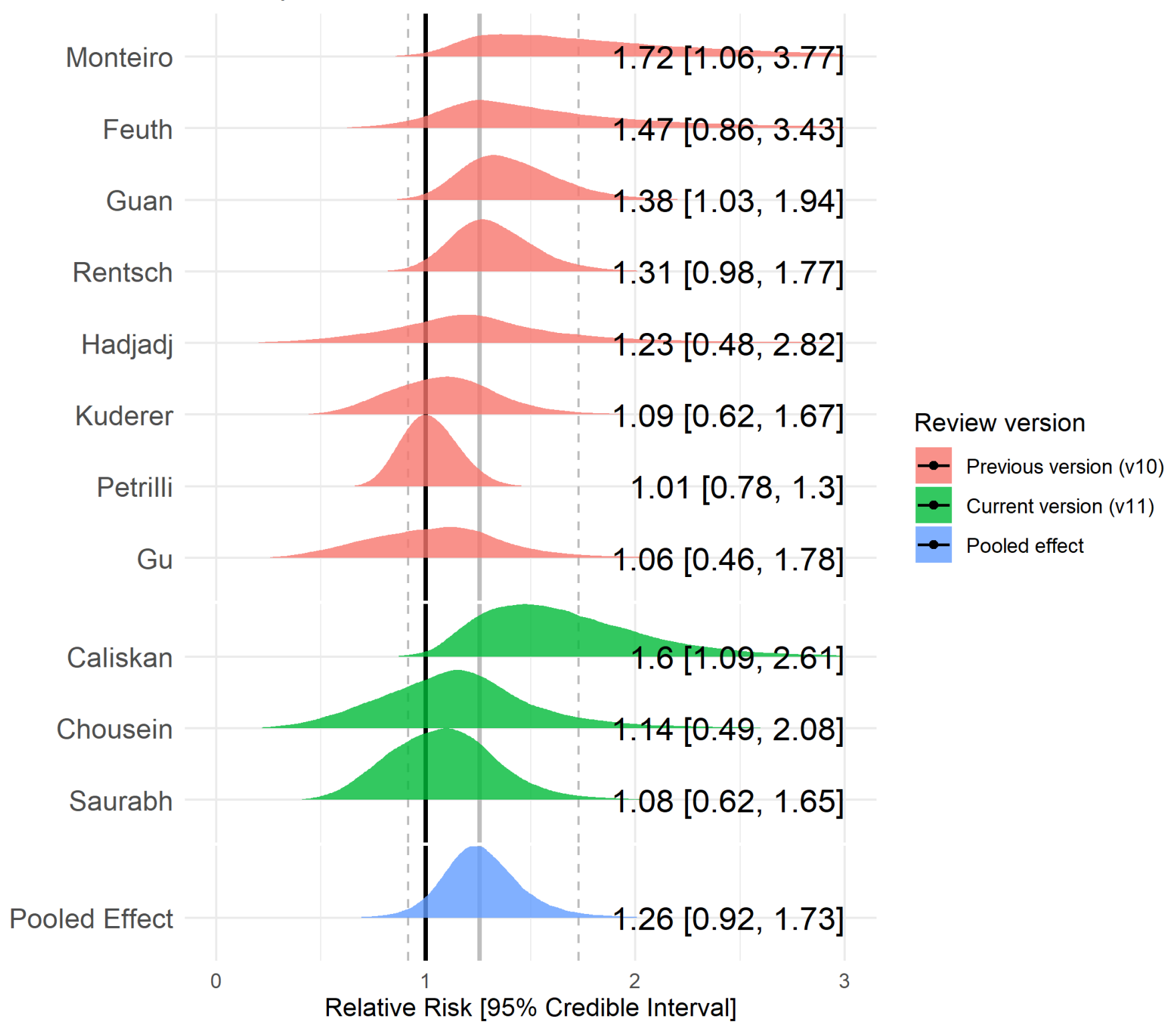

v10 severity prior

Figure 7. Forest plot for the risk of severe disease in current vs. never smokers. The prior from the previous review version (v10) was RR $=1.26$. 


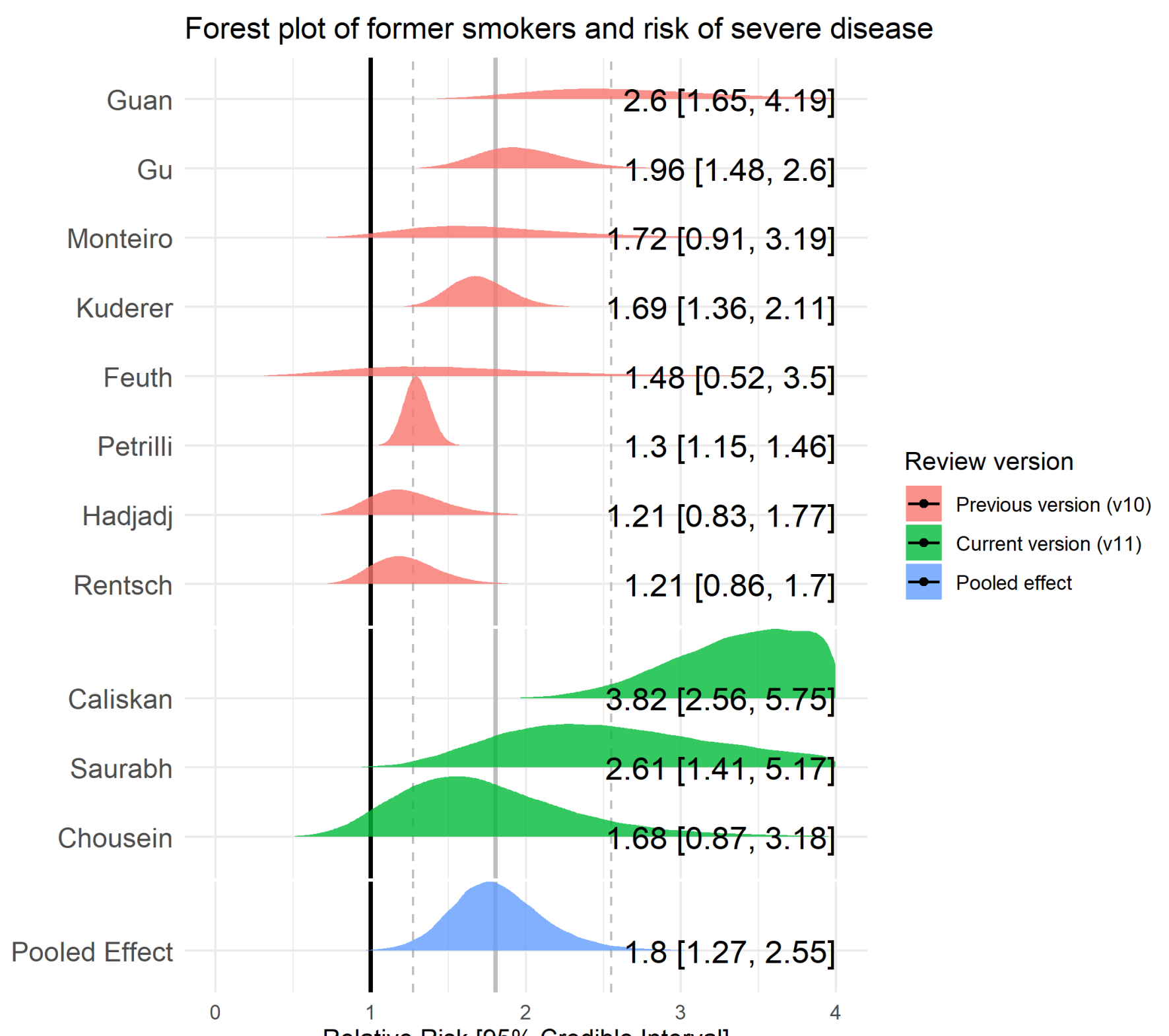

Relative Risk [95\% Credible Interval]

v10 severity prior

Figure 8. Forest plot for the risk of severe disease in former vs. never smokers. The prior from the previous review version $(\mathrm{v} 10)$ was $\mathrm{RR}=1.52$.

Mortality by smoking status

89 studies reported mortality from COVID-19 by smoking status (see Table 5), with 21 'fair' quality studies included in meta-analyses (see Figure 9 and 10). Current $(R R=1.12,95 \% \mathrm{Crl}=0.84-1.47, \tau=0.42)$ and former $(\mathrm{RR}=1.56,95 \% \mathrm{Crl}=$ 1.23-2, $\tau=0.43$ ) compared with never smokers were at increased risk of in-hospital mortality from COVID-19. However, data for current smokers were inconclusive and favoured there being no important association. The probability of current and former smokers being at greater risk of in-hospital mortality $(R R \geq 1.1)$ compared with never smokers was $60 \%$ and $>99 \%$, respectively. Results were materially unchanged in two sensitivity analyses. 
Table 5 COVID-19 attributed mortality by smoking status

\begin{tabular}{|c|c|c|c|c|c|c|c|c|c|c|c|}
\hline \multirow[b]{2}{*}{ Author } & \multirow[b]{2}{*}{$\begin{array}{l}\text { Population } \\
\text { with } \\
\text { mortality }\end{array}$} & \multicolumn{7}{|c|}{ Recovered } & \multicolumn{3}{|l|}{ Died } \\
\hline & & N (\%) & $\begin{array}{l}\text { Current } \\
\text { smoker } \\
(\%)\end{array}$ & $\begin{array}{l}\text { Former } \\
\text { smoker } \\
(\%)\end{array}$ & $\begin{array}{l}\text { Current/former } \\
\text { smoker (\%) }\end{array}$ & $\begin{array}{l}\text { Never } \\
\text { smoker } \\
(\%)\end{array}$ & $\begin{array}{l}\text { Never/unknown } \\
\text { smoker (\%) }\end{array}$ & $\begin{array}{l}\text { Not } \\
\text { stated } \\
(\%)\end{array}$ & N (\%) & $\begin{array}{l}\text { Current } \\
\text { smoker } \\
(\%)\end{array}$ & $\begin{array}{l}\text { Former } \\
\text { smoker } \\
(\%)\end{array}$ \\
\hline Chen & 274 & $\begin{array}{l}161 \\
(58 \%)\end{array}$ & $\begin{array}{l}5 \\
(3.11 \%)\end{array}$ & $\begin{array}{l}5 \\
(3.11 \%)\end{array}$ & - & - & - & $\begin{array}{l}151 \\
(93.79 \%)\end{array}$ & $\begin{array}{l}113 \\
(41 \%)\end{array}$ & $\begin{array}{l}7 \\
(6.19 \%)\end{array}$ & $\begin{array}{l}2 \\
(1.77 \%)\end{array}$ \\
\hline Zhou, Yu & 191 & $\begin{array}{l}137 \\
(71 \%)\end{array}$ & $\begin{array}{l}6 \\
(4.38 \%)\end{array}$ & - & - & - & - & $\begin{array}{l}131 \\
(95.62 \%)\end{array}$ & $\begin{array}{l}54 \\
(28 \%)\end{array}$ & $\begin{array}{l}5 \\
(9.26 \%)\end{array}$ & - \\
\hline Yang, Yu & 52 & $\begin{array}{l}20 \\
(38 \%)\end{array}$ & $\begin{array}{l}2 \\
(10.00 \%)\end{array}$ & - & - & - & $18(90.00 \%)$ & - & $\begin{array}{l}32 \\
(61 \%)\end{array}$ & - & - \\
\hline Borobia & 2,226 & $\begin{array}{l}1766 \\
(79 \%)\end{array}$ & $\begin{array}{l}113 \\
(6.40 \%)\end{array}$ & - & - & - & - & $\begin{array}{l}1653 \\
(93.60 \%)\end{array}$ & $\begin{array}{l}460 \\
(20 \%)\end{array}$ & $\begin{array}{l}44 \\
(9.57 \%)\end{array}$ & - \\
\hline Giacomelli & 233 & $\begin{array}{l}185 \\
(79 \%)\end{array}$ & - & - & $53(28.65 \%)$ & $\begin{array}{l}132 \\
(71.35 \%)\end{array}$ & - & - & $\begin{array}{l}48 \\
(20 \%)\end{array}$ & - & - \\
\hline Yao & 108 & $\begin{array}{l}96 \\
(88 \%)\end{array}$ & $\begin{array}{l}1 \\
(1.04 \%)\end{array}$ & - & - & - & - & $\begin{array}{l}95 \\
(98.96 \%)\end{array}$ & $\begin{array}{l}12 \\
(11 \%)\end{array}$ & $\begin{array}{l}3 \\
(25.00 \%)\end{array}$ & - \\
\hline Carillo-Vega & 9,946 & $\begin{array}{l}8983 \\
(90 \%)\end{array}$ & $\begin{array}{l}795 \\
(8.85 \%)\end{array}$ & - & - & - & - & $\begin{array}{l}8188 \\
(91.15 \%)\end{array}$ & $\begin{array}{l}963 \\
(9 \%)\end{array}$ & $\begin{array}{l}99 \\
(10.28 \%)\end{array}$ & - \\
\hline $\mathrm{Ge}$ & 51 & $\begin{array}{l}39 \\
(76 \%)\end{array}$ & $\begin{array}{l}6 \\
(15.38 \%)\end{array}$ & - & - & - & - & $\begin{array}{l}33 \\
(84.62 \%)\end{array}$ & $\begin{array}{l}12 \\
(23 \%)\end{array}$ & $\begin{array}{l}1 \\
(8.33 \%)\end{array}$ & - \\
\hline Chen, Jiang & 135 & $\begin{array}{l}\text { NA } \\
\text { (NA\%) }\end{array}$ & - & - & - & - & - & - & $\begin{array}{l}31 \\
(22 \%)\end{array}$ & - & - \\
\hline Heili-Frades & 4,712 & $\begin{array}{l}4086 \\
(86 \%)\end{array}$ & $\begin{array}{l}210 \\
(5.14 \%)\end{array}$ & $\begin{array}{l}659 \\
(16.13 \%)\end{array}$ & - & - & $3217(78.73 \%)$ & - & $\begin{array}{l}626 \\
(13 \%)\end{array}$ & $\begin{array}{l}23 \\
(3.67 \%)\end{array}$ & $\begin{array}{l}161 \\
(25.72 \%)\end{array}$ \\
\hline Kim, Garg & 2,490 & $\begin{array}{l}2070 \\
(83 \%)\end{array}$ & $\begin{array}{l}128 \\
(6.18 \%)\end{array}$ & $\begin{array}{l}481 \\
(23.24 \%)\end{array}$ & - & - & $1461(70.58 \%)$ & - & $\begin{array}{l}420 \\
(16 \%)\end{array}$ & $\begin{array}{l}22 \\
(5.24 \%)\end{array}$ & $\begin{array}{l}161 \\
(38.33 \%)\end{array}$ \\
\hline Al-Hindawi & 31 & $\begin{array}{l}15 \\
(48 \%)\end{array}$ & $\begin{array}{l}0 \\
(0.00 \%)\end{array}$ & $\begin{array}{l}10 \\
(66.67 \%)\end{array}$ & - & $\begin{array}{l}5 \\
(33.33 \%)\end{array}$ & - & - & $\begin{array}{l}16 \\
(51 \%)\end{array}$ & $\begin{array}{l}1 \\
(6.25 \%)\end{array}$ & $\begin{array}{l}12 \\
(75.00 \%)\end{array}$ \\
\hline Louis & 22 & $\begin{array}{l}16 \\
(72 \%)\end{array}$ & - & - & $7(43.75 \%)$ & - & - & $\begin{array}{l}9 \\
(56.25 \%)\end{array}$ & $\begin{array}{l}6 \\
(27 \%)\end{array}$ & - & - \\
\hline Soto-Mota & 400 & $\begin{array}{l}200 \\
(50 \%)\end{array}$ & - & - & 23 (11.50\%) & - & - & $\begin{array}{l}177 \\
(88.50 \%)\end{array}$ & $\begin{array}{l}200 \\
(50 \%)\end{array}$ & - & - \\
\hline Garibaldi & 747 & $\begin{array}{l}634 \\
(84 \%)\end{array}$ & $\begin{array}{l}36 \\
(5.68 \%)\end{array}$ & $\begin{array}{l}129 \\
(20.35 \%)\end{array}$ & - & - & - & $\begin{array}{l}469 \\
(73.97 \%)\end{array}$ & $\begin{array}{l}113 \\
(15 \%)\end{array}$ & $\begin{array}{l}6 \\
(5.31 \%)\end{array}$ & $\begin{array}{l}36 \\
(31.86 \%)\end{array}$ \\
\hline Docherty & 13,364 & $\begin{array}{l}8199 \\
(61 \%)\end{array}$ & $\begin{array}{l}370 \\
(4.51 \%)\end{array}$ & $\begin{array}{l}1832 \\
(22.34 \%)\end{array}$ & - & $\begin{array}{l}4179 \\
(50.97 \%)\end{array}$ & - & $\begin{array}{l}1818 \\
(22.17 \%)\end{array}$ & $\begin{array}{l}5165 \\
(38 \%)\end{array}$ & $\begin{array}{l}214 \\
(4.14 \%)\end{array}$ & $\begin{array}{l}1350 \\
(26.14 \%)\end{array}$ \\
\hline Kuderer & 928 & $\begin{array}{l}807 \\
(86 \%)\end{array}$ & $\begin{array}{l}38 \\
(4.71 \%)\end{array}$ & $\begin{array}{l}262 \\
(32.47 \%)\end{array}$ & - & $\begin{array}{l}425 \\
(52.66 \%)\end{array}$ & - & $\begin{array}{l}31 \\
(3.84 \%)\end{array}$ & $\begin{array}{l}121 \\
(13 \%)\end{array}$ & $\begin{array}{l}5 \\
(4.13 \%)\end{array}$ & $\begin{array}{l}64 \\
(52.89 \%)\end{array}$ \\
\hline Ramlall & 11,116 & $\begin{array}{l}10498 \\
(94 \%)\end{array}$ & - & - & 2771 (26.40\%) & $\begin{array}{l}7727 \\
(73.60 \%)\end{array}$ & - & - & $\begin{array}{l}618 \\
(5 \%)\end{array}$ & - & - \\
\hline Wang, Oekelen & 57 & $\begin{array}{l}43 \\
(75 \%)\end{array}$ & - & - & $14(32.56 \%)$ & - & - & $\begin{array}{l}29 \\
(67.44 \%)\end{array}$ & $\begin{array}{l}14 \\
(24 \%)\end{array}$ & - & - \\
\hline Martinez-Portilla & 224 & $\begin{array}{l}217 \\
(96 \%)\end{array}$ & - & - & 7 (3.23\%) & - & - & $\begin{array}{l}210 \\
(96.77 \%)\end{array}$ & $7(3 \%)$ & - & - \\
\hline Cen & 1,007 & $\begin{array}{l}964 \\
(95 \%)\end{array}$ & - & - & 87 (9.02\%) & - & - & $\begin{array}{l}877 \\
(90.98 \%)\end{array}$ & $\begin{array}{l}43 \\
(4 \%)\end{array}$ & - & - \\
\hline Klang & 3,406 & $\begin{array}{l}2270 \\
(66 \%)\end{array}$ & - & - & $492(21.67 \%)$ & - & - & $\begin{array}{l}1778 \\
(78.33 \%)\end{array}$ & $\begin{array}{l}1136 \\
(33 \%)\end{array}$ & - & - \\
\hline
\end{tabular}




\begin{tabular}{|c|c|c|c|c|c|c|c|c|c|c|c|}
\hline Wang, Zhong & 5,510 & $\begin{array}{l}48 / 4 \\
(88 \%)\end{array}$ & $\begin{array}{l}24 / \\
(5.07 \%)\end{array}$ & $\begin{array}{l}1083 \\
(22.22 \%)\end{array}$ & - & $\begin{array}{l}3544 \\
(72.71 \%)\end{array}$ & - & - & $\begin{array}{l}636 \\
(11 \%)\end{array}$ & $\begin{array}{l}28 \\
(4.40 \%)\end{array}$ & $\begin{array}{l}214 \\
(33.65 \%)\end{array}$ \\
\hline Miyara & 338 & $\begin{array}{l}211 \\
(62 \%)\end{array}$ & $\begin{array}{l}13 \\
(6.16 \%)\end{array}$ & $\begin{array}{l}58 \\
(27.49 \%)\end{array}$ & - & $\begin{array}{l}141 \\
(66.82 \%)\end{array}$ & - & - & $\begin{array}{l}46 \\
(13 \%)\end{array}$ & $\begin{array}{l}1 \\
(2.17 \%)\end{array}$ & $\begin{array}{l}23 \\
(50.00 \%)\end{array}$ \\
\hline Rajter & 255 & $\begin{array}{l}209 \\
(81 \%)\end{array}$ & - & - & $28(13.40 \%)$ & $\begin{array}{l}181 \\
(86.60 \%)\end{array}$ & - & - & $\begin{array}{l}53 \\
(20 \%)\end{array}$ & - & - \\
\hline Zeng & 1,031 & $\begin{array}{l}866 \\
(84 \%)\end{array}$ & - & - & $69(7.97 \%)$ & - & - & $\begin{array}{l}797 \\
(92.03 \%)\end{array}$ & $\begin{array}{l}165 \\
(16 \%)\end{array}$ & - & - \\
\hline Chen, Yu & 1,859 & $\begin{array}{l}1651 \\
(88 \%)\end{array}$ & $\begin{array}{l}32 \\
(1.94 \%)\end{array}$ & $\begin{array}{l}54 \\
(3.27 \%)\end{array}$ & - & $\begin{array}{l}1565 \\
(94.79 \%)\end{array}$ & - & - & $\begin{array}{l}208 \\
(11 \%)\end{array}$ & $\begin{array}{l}13 \\
(6.25 \%)\end{array}$ & $\begin{array}{l}12 \\
(5.77 \%)\end{array}$ \\
\hline Garassino & 190 & $\begin{array}{l}124 \\
(65 \%)\end{array}$ & - & - & $92(74.19 \%)$ & $\begin{array}{l}32 \\
(25.81 \%)\end{array}$ & - & - & $\begin{array}{l}66 \\
(34 \%)\end{array}$ & - & $\begin{array}{l}61 \\
(92.42 \%)\end{array}$ \\
\hline $\mathrm{Gu}$ & 884 & $\begin{array}{l}864 \\
(97 \%)\end{array}$ & $\begin{array}{l}40 \\
(4.63 \%)\end{array}$ & $\begin{array}{l}250 \\
(28.94 \%)\end{array}$ & - & $\begin{array}{l}219 \\
(25.35 \%)\end{array}$ & - & - & $\begin{array}{l}20 \\
(2 \%)\end{array}$ & $\begin{array}{l}0 \\
(0.00 \%)\end{array}$ & $\begin{array}{l}14 \\
(70.00 \%)\end{array}$ \\
\hline Zhou, He & - & $\begin{array}{l}\text { NA } \\
\text { (NA\%) }\end{array}$ & - & - & - & - & - & - & $\begin{array}{l}\text { NA } \\
\text { (NA\%) }\end{array}$ & - & - \\
\hline Sigel & 88 & $\begin{array}{l}70 \\
(79 \%)\end{array}$ & - & - & 37 (52.86\%) & - & - & $\begin{array}{l}33 \\
(47.14 \%)\end{array}$ & $\begin{array}{l}18 \\
(20 \%)\end{array}$ & - & - \\
\hline Nguyen & 356 & $\begin{array}{l}308 \\
(86 \%)\end{array}$ & - & - & $91(29.55 \%)$ & - & - & $\begin{array}{l}217 \\
(70.45 \%)\end{array}$ & $\begin{array}{l}45 \\
(12 \%)\end{array}$ & - & - \\
\hline de Souza & 8,443 & $\begin{array}{l}7826 \\
(92 \%)\end{array}$ & - & - & $95(1.21 \%)$ & - & $7571(96.74 \%)$ & $\begin{array}{l}160 \\
(2.04 \%)\end{array}$ & $\begin{array}{l}617 \\
(7 \%)\end{array}$ & - & - \\
\hline Mendy & 532 & $\begin{array}{l}663 \\
(124 \%)\end{array}$ & - & - & $160(24.13 \%)$ & - & - & $\begin{array}{l}503 \\
(75.87 \%)\end{array}$ & $\begin{array}{l}26 \\
(4 \%)\end{array}$ & - & - \\
\hline Shi, Resurreccion & 256 & $\begin{array}{l}210 \\
(82 \%)\end{array}$ & - & - & $128(60.95 \%)$ & - & - & $\begin{array}{l}82 \\
(39.05 \%)\end{array}$ & $\begin{array}{l}46 \\
(17 \%)\end{array}$ & - & - \\
\hline Xie & 619 & $\begin{array}{l}591 \\
(95 \%)\end{array}$ & - & - & 43 (7.28\%) & - & - & $\begin{array}{l}548 \\
(92.72 \%)\end{array}$ & $\begin{array}{l}28 \\
(4 \%)\end{array}$ & - & - \\
\hline Fox & 54 & $\begin{array}{l}35 \\
(64 \%)\end{array}$ & $\begin{array}{l}1 \\
(2.86 \%)\end{array}$ & $\begin{array}{l}4 \\
(11.43 \%)\end{array}$ & - & $\begin{array}{l}18 \\
(51.43 \%)\end{array}$ & - & $\begin{array}{l}12 \\
(34.29 \%)\end{array}$ & $\begin{array}{l}19 \\
(35 \%)\end{array}$ & $\begin{array}{l}0 \\
(0.00 \%)\end{array}$ & $\begin{array}{l}2 \\
(10.53 \%)\end{array}$ \\
\hline Zhang, Cao & 289 & $\begin{array}{l}240 \\
(83 \%)\end{array}$ & $\begin{array}{l}10 \\
(4.17 \%)\end{array}$ & $\begin{array}{l}6 \\
(2.50 \%)\end{array}$ & - & - & - & $\begin{array}{l}224 \\
(93.33 \%)\end{array}$ & $\begin{array}{l}49 \\
(16 \%)\end{array}$ & $\begin{array}{l}4 \\
(8.16 \%)\end{array}$ & $\begin{array}{l}8 \\
(16.33 \%)\end{array}$ \\
\hline Gupta & 496 & $\begin{array}{l}255 \\
(51 \%)\end{array}$ & - & - & $15(5.88 \%)$ & - & 80 (31.37\%) & $\begin{array}{l}160 \\
(62.75 \%)\end{array}$ & $\begin{array}{l}241 \\
(48 \%)\end{array}$ & - & - \\
\hline Soares & 1,152 & $\begin{array}{l}696 \\
(60 \%)\end{array}$ & $\begin{array}{l}38 \\
(5.46 \%)\end{array}$ & - & - & - & 658 (94.54\%) & - & $\begin{array}{l}456 \\
(39 \%)\end{array}$ & $\begin{array}{l}39 \\
(8.55 \%)\end{array}$ & - \\
\hline Thompson & 470 & $\begin{array}{l}301 \\
(64 \%)\end{array}$ & $\begin{array}{l}39 \\
(12.96 \%)\end{array}$ & $\begin{array}{l}79 \\
(26.25 \%)\end{array}$ & - & $\begin{array}{l}183 \\
(60.80 \%)\end{array}$ & - & - & $\begin{array}{l}169 \\
(35 \%)\end{array}$ & $\begin{array}{l}27 \\
(15.98 \%)\end{array}$ & $\begin{array}{l}49 \\
(28.99 \%)\end{array}$ \\
\hline Bernaola & 1,645 & $\begin{array}{l}1382 \\
(84 \%)\end{array}$ & $\begin{array}{l}35 \\
(2.53 \%)\end{array}$ & $\begin{array}{l}146 \\
(10.56 \%)\end{array}$ & - & $\begin{array}{l}1201 \\
(86.90 \%)\end{array}$ & - & - & $\begin{array}{l}263 \\
(15 \%)\end{array}$ & $\begin{array}{l}6 \\
(2.28 \%)\end{array}$ & $\begin{array}{l}33 \\
(12.55 \%)\end{array}$ \\
\hline Islam & 654 & $\begin{array}{l}631 \\
(96 \%)\end{array}$ & $\begin{array}{l}103 \\
(16.32 \%)\end{array}$ & - & - & - & - & $\begin{array}{l}507 \\
(80.35 \%)\end{array}$ & $\begin{array}{l}23 \\
(3 \%)\end{array}$ & $\begin{array}{l}3 \\
(13.04 \%)\end{array}$ & - \\
\hline Philipose & 466 & $\begin{array}{l}267 \\
(57 \%)\end{array}$ & $\begin{array}{l}19 \\
(7.12 \%)\end{array}$ & $\begin{array}{l}204 \\
(76.40 \%)\end{array}$ & - & $\begin{array}{l}44 \\
(16.48 \%)\end{array}$ & - & - & $\begin{array}{l}199 \\
(42 \%)\end{array}$ & $\begin{array}{l}9 \\
(4.52 \%)\end{array}$ & $\begin{array}{l}137 \\
(68.84 \%)\end{array}$ \\
\hline Fillmore & 1,794 & $\begin{array}{l}1566 \\
(87 \%)\end{array}$ & $\begin{array}{l}408 \\
(26.05 \%)\end{array}$ & $\begin{array}{l}758 \\
(48.40 \%)\end{array}$ & - & $\begin{array}{l}279 \\
(17.82 \%)\end{array}$ & - & $\begin{array}{l}98 \\
(6.26 \%)\end{array}$ & $\begin{array}{l}228 \\
(12 \%)\end{array}$ & $\begin{array}{l}44 \\
(19.30 \%)\end{array}$ & $\begin{array}{l}141 \\
(61.84 \%)\end{array}$ \\
\hline Pan & 3,536 & $\begin{array}{l}3302 \\
(93 \%)\end{array}$ & - & - & $862(26.11 \%)$ & - & - & $\begin{array}{l}2440 \\
(73.89 \%)\end{array}$ & $\begin{array}{l}234 \\
(6 \%)\end{array}$ & - & - \\
\hline Zhao, Chen & 474 & $\begin{array}{l}398 \\
(83 \%)\end{array}$ & $\begin{array}{l}87 \\
(21.86 \%)\end{array}$ & - & - & - & - & $\begin{array}{l}311 \\
(78.14 \%)\end{array}$ & $\begin{array}{l}82 \\
(17 \%)\end{array}$ & $\begin{array}{l}36 \\
(43.90 \%)\end{array}$ & - \\
\hline
\end{tabular}




\begin{tabular}{|c|c|c|c|c|c|c|c|c|c|c|c|}
\hline ' יויו" & ルেンט & (NA\%) & & & & & & & $(100 \%)$ & $(5.54 \%)$ & $(42.62 \%)$ \\
\hline Chand & 300 & $\begin{array}{l}143 \\
(47 \%)\end{array}$ & $\begin{array}{l}23 \\
(16.08 \%)\end{array}$ & - & - & - & - & $\begin{array}{l}120 \\
(83.92 \%)\end{array}$ & $\begin{array}{l}157 \\
(52 \%)\end{array}$ & $\begin{array}{l}44 \\
(28.03 \%)\end{array}$ & - \\
\hline Oliveira & 131 & $\begin{array}{l}105 \\
(80 \%)\end{array}$ & - & - & 16 (15.24\%) & - & $83(79.05 \%)$ & $6(5.71 \%)$ & $\begin{array}{l}26 \\
(19 \%)\end{array}$ & - & - \\
\hline Vilar-Garcia & 328,892 & $\begin{array}{l}316605 \\
(96 \%)\end{array}$ & $\begin{array}{l}71215 \\
(22.49 \%)\end{array}$ & - & - & - & - & $\begin{array}{l}245390 \\
(77.51 \%)\end{array}$ & $\begin{array}{l}12287 \\
(3 \%)\end{array}$ & $\begin{array}{l}3103 \\
(25.25 \%)\end{array}$ & - \\
\hline lbarra-Nava & 416,546 & $\begin{array}{l}370038 \\
(88 \%)\end{array}$ & $\begin{array}{l}27001 \\
(7.30 \%)\end{array}$ & - & - & - & - & $\begin{array}{l}343037 \\
(92.70 \%)\end{array}$ & $\begin{array}{l}46508 \\
(11 \%)\end{array}$ & $\begin{array}{l}3817 \\
(8.21 \%)\end{array}$ & - \\
\hline Rubio-Rivas & 186 & $\begin{array}{l}147 \\
(79 \%)\end{array}$ & $\begin{array}{l}7 \\
(4.76 \%)\end{array}$ & $\begin{array}{l}32 \\
(21.77 \%)\end{array}$ & - & $\begin{array}{l}108 \\
(73.47 \%)\end{array}$ & - & - & $\begin{array}{l}39 \\
(20 \%)\end{array}$ & $\begin{array}{l}1 \\
(2.56 \%)\end{array}$ & $\begin{array}{l}6 \\
(15.38 \%)\end{array}$ \\
\hline Ren & 432 & $\begin{array}{l}289 \\
(66 \%)\end{array}$ & $\begin{array}{l}25 \\
(8.65 \%)\end{array}$ & - & - & $\begin{array}{l}264 \\
(91.35 \%)\end{array}$ & - & - & $\begin{array}{l}143 \\
(33 \%)\end{array}$ & $\begin{array}{l}18 \\
(12.59 \%)\end{array}$ & - \\
\hline Ullah & 212 & $\begin{array}{l}158 \\
(74 \%)\end{array}$ & $\begin{array}{l}22 \\
(13.92 \%)\end{array}$ & $\begin{array}{l}67 \\
(42.41 \%)\end{array}$ & - & $\begin{array}{l}63 \\
(39.87 \%)\end{array}$ & - & $6(3.80 \%)$ & $\begin{array}{l}54 \\
(25 \%)\end{array}$ & $\begin{array}{l}2 \\
(3.70 \%)\end{array}$ & $\begin{array}{l}35 \\
(64.81 \%)\end{array}$ \\
\hline Dashti & 3,401 & $\begin{array}{l}2892 \\
(85 \%)\end{array}$ & $\begin{array}{l}190 \\
(6.57 \%)\end{array}$ & $\begin{array}{l}689 \\
(23.82 \%)\end{array}$ & - & $\begin{array}{l}1756 \\
(60.72 \%)\end{array}$ & - & $\begin{array}{l}257 \\
(8.89 \%)\end{array}$ & $\begin{array}{l}509 \\
(14 \%)\end{array}$ & $\begin{array}{l}20 \\
(3.93 \%)\end{array}$ & $\begin{array}{l}171 \\
(33.60 \%)\end{array}$ \\
\hline Nicholson & 1,040 & $\begin{array}{l}829 \\
(79 \%)\end{array}$ & $\begin{array}{l}70 \\
(8.44 \%)\end{array}$ & $\begin{array}{l}163 \\
(19.66 \%)\end{array}$ & - & $\begin{array}{l}320 \\
(38.60 \%)\end{array}$ & - & $\begin{array}{l}276 \\
(33.29 \%)\end{array}$ & $\begin{array}{l}211 \\
(20 \%)\end{array}$ & $\begin{array}{l}16 \\
(7.58 \%)\end{array}$ & $\begin{array}{l}68 \\
(32.23 \%)\end{array}$ \\
\hline Kalan & 193 & $\begin{array}{l}188 \\
(97 \%)\end{array}$ & $\begin{array}{l}14 \\
(7.45 \%)\end{array}$ & - & - & $\begin{array}{l}162 \\
(86.17 \%)\end{array}$ & - & $\begin{array}{l}12 \\
(6.38 \%)\end{array}$ & $5(2 \%)$ & $\begin{array}{l}0 \\
(0.00 \%)\end{array}$ & - \\
\hline Incerti & 13,658 & $\begin{array}{l}11495 \\
(84 \%)\end{array}$ & $\begin{array}{l}785 \\
(6.83 \%)\end{array}$ & $\begin{array}{l}2450 \\
(21.31 \%)\end{array}$ & - & $\begin{array}{l}5450 \\
(47.41 \%)\end{array}$ & $2810(24.45 \%)$ & - & $\begin{array}{l}2163 \\
(15 \%)\end{array}$ & $\begin{array}{l}81 \\
(3.74 \%)\end{array}$ & $\begin{array}{l}642 \\
(29.68 \%)\end{array}$ \\
\hline loannou & 10,131 & $\begin{array}{l}9033 \\
(89 \%)\end{array}$ & $\begin{array}{l}1054 \\
(11.67 \%)\end{array}$ & $\begin{array}{l}3549 \\
(39.29 \%)\end{array}$ & - & $\begin{array}{l}3339 \\
(36.96 \%)\end{array}$ & - & $\begin{array}{l}1091 \\
(12.08 \%)\end{array}$ & $\begin{array}{l}1098 \\
(10 \%)\end{array}$ & $\begin{array}{l}81 \\
(7.38 \%)\end{array}$ & $\begin{array}{l}528 \\
(48.09 \%)\end{array}$ \\
\hline Lamure & 89 & $\begin{array}{l}59 \\
(66 \%)\end{array}$ & $\begin{array}{l}4 \\
(6.78 \%)\end{array}$ & $\begin{array}{l}16 \\
(27.12 \%)\end{array}$ & - & $\begin{array}{l}31 \\
(52.54 \%)\end{array}$ & - & $\begin{array}{l}8 \\
(13.56 \%)\end{array}$ & $\begin{array}{l}30 \\
(33 \%)\end{array}$ & $\begin{array}{l}1 \\
(3.33 \%)\end{array}$ & $\begin{array}{l}13 \\
(43.33 \%)\end{array}$ \\
\hline Yadaw & 5,051 & $\begin{array}{l}4635 \\
(91 \%)\end{array}$ & $\begin{array}{l}162 \\
(3.50 \%)\end{array}$ & $\begin{array}{l}709 \\
(15.30 \%)\end{array}$ & - & $\begin{array}{l}2394 \\
(51.65 \%)\end{array}$ & - & $\begin{array}{l}1370 \\
(29.56 \%)\end{array}$ & $\begin{array}{l}416 \\
(8 \%)\end{array}$ & $\begin{array}{l}17 \\
(4.09 \%)\end{array}$ & $\begin{array}{l}105 \\
(25.24 \%)\end{array}$ \\
\hline Zinellu & 105 & $\begin{array}{l}77 \\
(73 \%)\end{array}$ & $\begin{array}{l}24 \\
(31.17 \%)\end{array}$ & $\begin{array}{l}8 \\
(10.39 \%)\end{array}$ & - & $\begin{array}{l}45 \\
(58.44 \%)\end{array}$ & - & - & $\begin{array}{l}28 \\
(26 \%)\end{array}$ & $\begin{array}{l}8 \\
(28.57 \%)\end{array}$ & $\begin{array}{l}3 \\
(10.71 \%)\end{array}$ \\
\hline Zhang, Li & 399 & $\begin{array}{l}\text { NA } \\
\text { (NA\%) }\end{array}$ & - & - & - & - & - & - & $\begin{array}{l}399 \\
(100 \%)\end{array}$ & $\begin{array}{l}60 \\
(15.04 \%)\end{array}$ & $\begin{array}{l}186 \\
(46.62 \%)\end{array}$ \\
\hline Wang, Shu & 59 & $\begin{array}{l}18 \\
(30 \%)\end{array}$ & - & - & $0(0.00 \%)$ & - & - & $\begin{array}{l}18 \\
(100.00 \%)\end{array}$ & $\begin{array}{l}41 \\
(69 \%)\end{array}$ & - & - \\
\hline Wang, Zheutlin & 2,448 & $\begin{array}{l}1706 \\
(69 \%)\end{array}$ & $\begin{array}{l}57 \\
(3.34 \%)\end{array}$ & $\begin{array}{l}315 \\
(18.46 \%)\end{array}$ & - & $\begin{array}{l}954 \\
(55.92 \%)\end{array}$ & - & $\begin{array}{l}380 \\
(22.27 \%)\end{array}$ & $\begin{array}{l}742 \\
(30 \%)\end{array}$ & $\begin{array}{l}25 \\
(3.37 \%)\end{array}$ & $\begin{array}{l}197 \\
(26.55 \%)\end{array}$ \\
\hline Torres-Macho & 1,968 & $\begin{array}{l}1643 \\
(83 \%)\end{array}$ & - & - & $335(20.39 \%)$ & - & - & $\begin{array}{l}1308 \\
(79.61 \%)\end{array}$ & $\begin{array}{l}325 \\
(16 \%)\end{array}$ & - & - \\
\hline Raines & 440 & $\begin{array}{l}408 \\
(92 \%)\end{array}$ & - & - & $222(54.41 \%)$ & $\begin{array}{l}186 \\
(45.59 \%)\end{array}$ & - & - & $\begin{array}{l}32 \\
(7 \%)\end{array}$ & - & - \\
\hline Parra-Bracamonte & 331,298 & $\begin{array}{l}292988 \\
(88 \%)\end{array}$ & - & - & 21269 (7.26\%) & - & - & $\begin{array}{l}271719 \\
(92.74 \%)\end{array}$ & $\begin{array}{l}38310 \\
(11 \%)\end{array}$ & - & - \\
\hline Li, Long, Zhang & 954 & $\begin{array}{l}876 \\
(91 \%)\end{array}$ & - & - & $48(5.48 \%)$ & $\begin{array}{l}828 \\
(94.52 \%)\end{array}$ & - & - & $\begin{array}{l}78 \\
(8 \%)\end{array}$ & - & - \\
\hline Bellan & 407 & $\begin{array}{l}285 \\
(70 \%)\end{array}$ & $\begin{array}{l}30 \\
(10.53 \%)\end{array}$ & - & - & - & $191(67.02 \%)$ & $\begin{array}{l}64 \\
(22.46 \%)\end{array}$ & $\begin{array}{l}122 \\
(29 \%)\end{array}$ & $\begin{array}{l}24 \\
(19.67 \%)\end{array}$ & - \\
\hline Alharthy & 352 & $\begin{array}{l}239 \\
(67 \%)\end{array}$ & $\begin{array}{l}109 \\
(45.61 \%)\end{array}$ & - & - & - & $130(54.39 \%)$ & - & $\begin{array}{l}113 \\
(32 \%)\end{array}$ & $\begin{array}{l}65 \\
(57.52 \%)\end{array}$ & - \\
\hline Adrish & 1,173 & $\begin{array}{l}783 \\
(66 \%)\end{array}$ & - & - & 205 (26.18\%) & $\begin{array}{l}578 \\
(73.82 \%)\end{array}$ & - & - & $\begin{array}{l}390 \\
(33 \%)\end{array}$ & - & - \\
\hline
\end{tabular}




\begin{tabular}{|c|c|c|c|c|c|c|c|c|c|c|c|}
\hline $\begin{array}{l}\text { Hoertel, Sanchez, } \\
\text { Vernet }\end{array}$ & 12,210 & $\begin{array}{l}12210 \\
(100 \%)\end{array}$ & $\begin{array}{l}898 \\
(7.35 \%)\end{array}$ & - & - & - & - & $\begin{array}{l}9904 \\
(81.11 \%)\end{array}$ & $\begin{array}{l}1408 \\
(11 \%)\end{array}$ & $\begin{array}{l}204 \\
(14.49 \%)\end{array}$ & - \\
\hline Sourij & 238 & $\begin{array}{l}180 \\
(75 \%)\end{array}$ & $\begin{array}{l}4 \\
(2.22 \%)\end{array}$ & $\begin{array}{l}26 \\
(14.44 \%)\end{array}$ & - & $\begin{array}{l}150 \\
(83.33 \%)\end{array}$ & - & - & $\begin{array}{l}58 \\
(24 \%)\end{array}$ & $\begin{array}{l}0 \\
(0.00 \%)\end{array}$ & $\begin{array}{l}12 \\
(20.69 \%)\end{array}$ \\
\hline Simons & 446 & $\begin{array}{l}318 \\
(71 \%)\end{array}$ & $\begin{array}{l}30 \\
(9.43 \%)\end{array}$ & $\begin{array}{l}109 \\
(34.28 \%)\end{array}$ & - & $\begin{array}{l}179 \\
(56.29 \%)\end{array}$ & - & - & $\begin{array}{l}128 \\
(28 \%)\end{array}$ & $\begin{array}{l}12 \\
(9.38 \%)\end{array}$ & $\begin{array}{l}63 \\
(49.22 \%)\end{array}$ \\
\hline Bisso & 168 & $\begin{array}{l}122 \\
(72 \%)\end{array}$ & $\begin{array}{l}13 \\
(10.66 \%)\end{array}$ & - & - & - & - & $\begin{array}{l}109 \\
(89.34 \%)\end{array}$ & $\begin{array}{l}46 \\
(27 \%)\end{array}$ & $\begin{array}{l}5 \\
(10.87 \%)\end{array}$ & - \\
\hline Caliskan & 565 & $\begin{array}{l}490 \\
(86 \%)\end{array}$ & $\begin{array}{l}97 \\
(19.80 \%)\end{array}$ & $\begin{array}{l}48 \\
(9.80 \%)\end{array}$ & - & $\begin{array}{l}345 \\
(70.41 \%)\end{array}$ & - & - & $\begin{array}{l}75 \\
(13 \%)\end{array}$ & $\begin{array}{l}21 \\
(28.00 \%)\end{array}$ & $\begin{array}{l}31 \\
(41.33 \%)\end{array}$ \\
\hline Ebrahimian & 226 & $\begin{array}{l}128 \\
(56 \%)\end{array}$ & - & - & $9(7.03 \%)$ & - & 119 (92.97\%) & - & $\begin{array}{l}98 \\
(43 \%)\end{array}$ & - & - \\
\hline Estiri & 16,709 & $\begin{array}{l}15879 \\
(95 \%)\end{array}$ & - & - & 1304 (8.21\%) & - & - & $\begin{array}{l}14575 \\
(91.79 \%)\end{array}$ & $\begin{array}{l}830 \\
(4 \%)\end{array}$ & - & - \\
\hline Ferrari & 198 & $\begin{array}{l}165 \\
(83 \%)\end{array}$ & - & - & 27 (16.36\%) & $\begin{array}{l}138 \\
(83.64 \%)\end{array}$ & - & - & $\begin{array}{l}33 \\
(16 \%)\end{array}$ & - & - \\
\hline Ho, Narasimhan & 4,909 & $\begin{array}{l}3553 \\
(72 \%)\end{array}$ & $\begin{array}{l}163 \\
(4.59 \%)\end{array}$ & $\begin{array}{l}743 \\
(20.91 \%)\end{array}$ & - & $\begin{array}{l}2647 \\
(74.50 \%)\end{array}$ & - & - & $\begin{array}{l}1356 \\
(27 \%)\end{array}$ & $\begin{array}{l}54 \\
(3.98 \%)\end{array}$ & $\begin{array}{l}319 \\
(23.53 \%)\end{array}$ \\
\hline Mansour & 111 & $\begin{array}{l}83 \\
(74 \%)\end{array}$ & $\begin{array}{l}5 \\
(6.02 \%)\end{array}$ & - & - & - & - & $\begin{array}{l}78 \\
(93.98 \%)\end{array}$ & $\begin{array}{l}28 \\
(25 \%)\end{array}$ & $\begin{array}{l}6 \\
(21.43 \%)\end{array}$ & - \\
\hline Park & 2,269 & $\begin{array}{l}2105 \\
(92 \%)\end{array}$ & $\begin{array}{l}92 \\
(4.37 \%)\end{array}$ & - & - & - & - & $\begin{array}{l}2013 \\
(95.63 \%)\end{array}$ & $\begin{array}{l}164 \\
(7 \%)\end{array}$ & $\begin{array}{l}2 \\
(1.22 \%)\end{array}$ & - \\
\hline Quan & 2,038 & $\begin{array}{l}1587 \\
(77 \%)\end{array}$ & - & - & 589 (37.11\%) & - & - & $\begin{array}{l}998 \\
(62.89 \%)\end{array}$ & $\begin{array}{l}442 \\
(21 \%)\end{array}$ & - & - \\
\hline Saurabh & 911 & $\begin{array}{l}870 \\
(95 \%)\end{array}$ & $\begin{array}{l}70 \\
(8.05 \%)\end{array}$ & $\begin{array}{l}4 \\
(0.46 \%)\end{array}$ & - & $\begin{array}{l}796 \\
(91.49 \%)\end{array}$ & - & - & $\begin{array}{l}41 \\
(4 \%)\end{array}$ & $\begin{array}{l}4 \\
(9.76 \%)\end{array}$ & $\begin{array}{l}2 \\
(4.88 \%)\end{array}$ \\
\hline Strangfeld & 3,729 & $\begin{array}{l}3339 \\
(89 \%)\end{array}$ & - & - & 664 (19.89\%) & $\begin{array}{l}2190 \\
(65.59 \%)\end{array}$ & - & $\begin{array}{l}485 \\
(14.53 \%)\end{array}$ & $\begin{array}{l}390 \\
(10 \%)\end{array}$ & - & - \\
\hline Nezhadmoghadam & 13,367 & $\begin{array}{l}7757 \\
(58 \%)\end{array}$ & $\begin{array}{l}686 \\
(8.84 \%)\end{array}$ & - & - & - & - & $\begin{array}{l}7071 \\
(91.16 \%)\end{array}$ & $\begin{array}{l}5610 \\
(41 \%)\end{array}$ & $\begin{array}{l}565 \\
(10.07 \%)\end{array}$ & - \\
\hline Nuno & 4,730 & $\begin{array}{l}4401 \\
(93 \%)\end{array}$ & $\begin{array}{l}427 \\
(9.70 \%)\end{array}$ & - & - & - & - & $\begin{array}{l}3974 \\
(90.30 \%)\end{array}$ & $\begin{array}{l}329 \\
(6 \%)\end{array}$ & $\begin{array}{l}53 \\
(16.11 \%)\end{array}$ & - \\
\hline
\end{tabular}


Forest plot of current smokers and mortality

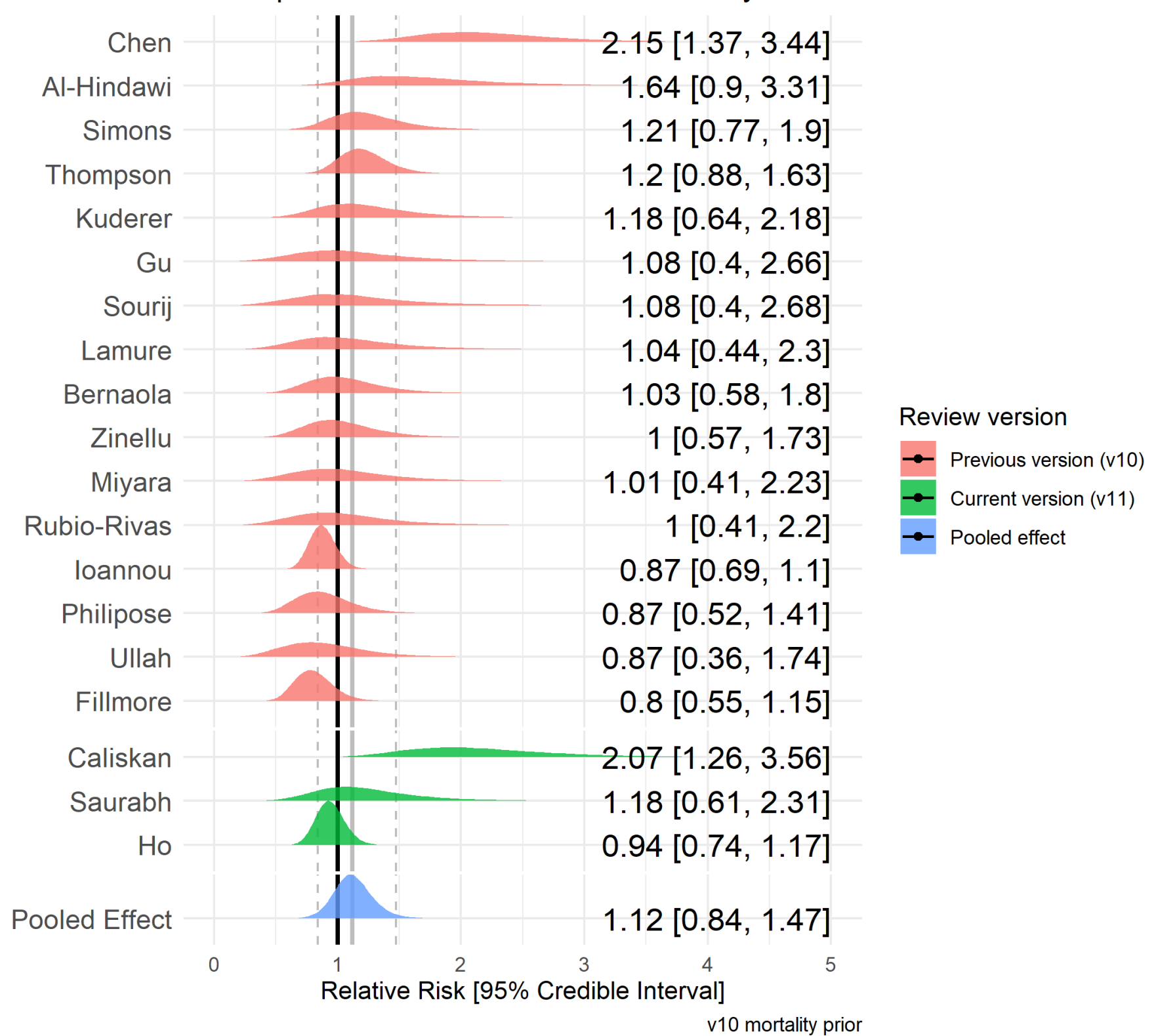

Figure 9. Forest plot for the risk of mortality in current vs. never smokers. The prior from the previous review version (v10) was RR $=1.05$. 


\section{Forest plot of former smokers and mortality}

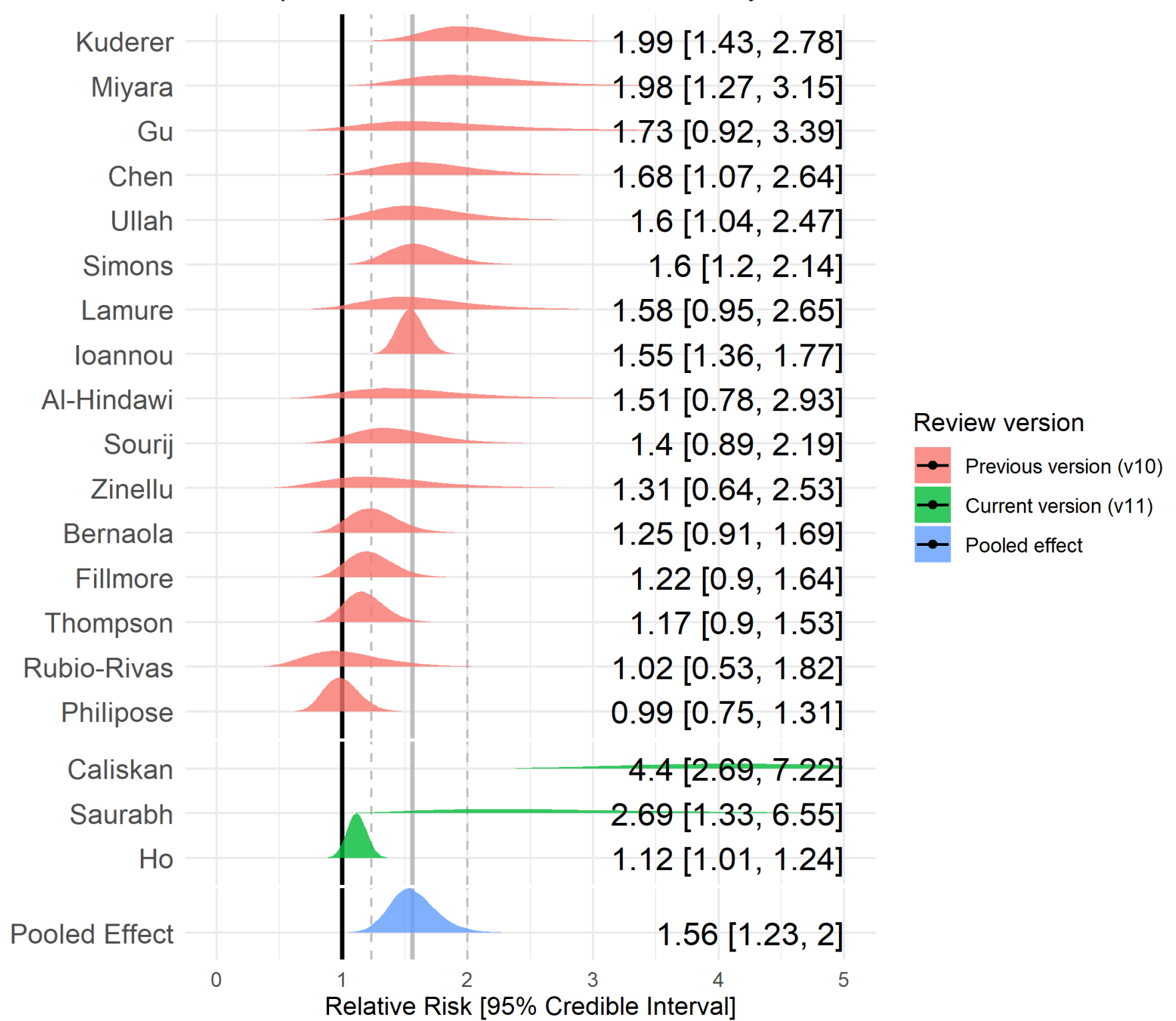

v10 mortality prior

Figure 10. Forest plot for the risk of mortality in former smokers vs. never smokers. The prior from the previous version (v10) was RR $=1.40$.

\section{Discussion}

This living rapid review found uncertainty in the majority of 405 studies arising from the recording of smoking status. Notwithstanding this uncertainty, compared with overall adult national prevalence estimates, recorded current smoking rates in most studies were lower than expected. In a subset of good and fair quality studies ( $n=30)$, current but not former smokers had a reduced risk of testing positive for SARS-CoV-2 but current smokers appeared somewhat more likely to present for testing and/or receive a test. Data for current smokers on the risk of hospitalisation, disease severity and mortality were inconclusive, and favoured there being no important associations with hospitalisation and mortality and a small but important increase in the risk of severe disease. Former smokers were at increased risk of hospitalisation, 
disease severity and mortality compared with never smokers.

Issues complicating interpretation

Interpretation of results from studies conducted during the first phase of the SARS-CoV-2 pandemic is complicated by several factors (see Figure 11):

1. Exposure to SARS-CoV-2

i) Exposure to the SARS-CoV-2 virus is heterogeneous with different subgroups at heightened risk of infection at different stages of the pandemic, at least partly due to differential contact matrices by age, sex and socioeconomic position (CMMID COVID-19 working group et al., 2020), which are associated with smoking status.

ii) The probability of viral exposure depends largely on local prevalence, which varies over time. This likely introduces bias in studies assessing the rate of infection by smoking status conducted in the early phase of the pandemic.

2. Infection with SARS-CoV-2

i) Infection following viral exposure depends on individual differences in, for example, genetic susceptibility or immunocompetence, which are poorly understood at present and may be confounded with smoking. For example, the household secondary attack rate for COVID-19 is estimated at 17\% (Fung et al., 2020).

ii) Heated and humidified air may act to disrupt the ability of the virus to persist in the airway mucosa of smokers. There is some evidence that transient localised hyperthermia can inhibit replication of rhinoviruses, a non-enveloped virus that causes the common cold (Conti et al., 1999). However, as SARS-CoV-2 is an enveloped virus (Schoeman and Fielding, 2019), it is unclear whether a similar protective effect against viral replication or invasion by heated and humidified air may occur.

3. Symptomatic COVID-19

i) An estimated $20 \%(95 \% \mathrm{Cl}=17-25 \%)$ of COVID-19 cases are asymptomatic (Buitrago-Garcia et al., 2020), with some evidence suggesting younger people are more likely to be asymptomatic (Kronbichler et al., 2020). Testing is hence likely limited in some subgroups, with the potential for these groups to include an overrepresentation of current smokers.

ii) On the other hand, current and former smokers may be more likely to meet local criteria for community testing due to increased prevalence of symptoms consistent with SARS-CoV-2 infection, such as cough, increased sputum production or altered sense of smell or taste (Hopkinson et al., 2020). Evidence from a small number of studies indicates that current smokers may be more likely to present for testing, hence increasing the denominator in comparisons with never smokers and potentially inflating the rate of negative tests in current smokers. Infection positivity rates estimated among random samples are more informative. We identified one population study conducted in Hungary reporting on seroprevalence and smoking status (Merkely et al., 2020); however, the response rate was only $58.8 \%$ and the current smoking rate was 10 percentage points below national prevalence estimates, which raises some doubt about representativeness of the final sample. Similarly, two further representative population surveys (Carrat et al., 2020; Richard et al., 2020) reported a current smoking rate of more than 10 percentage points below national prevalence ( $12 \%$ vs. $25 \%$ and $15 \%$ vs. $27 \%$ daily smoking prevalence, respectively) (Andler, 2019; FSO, 
2020).

\section{Testing positive for SARS-CoV-2}

i) Smokers with COVID-19 may be less likely to receive a SARS-CoV-2 test or present to hospital due to lack of access to healthcare and may be more likely to die in the community from sudden complications (i.e. self-selection bias) and thus not be recorded (Brown, 2020).

ii) Diagnostic criteria for SARS-CoV-2 infection and COVID-19 have changed during the course of the pandemic (Organisation, n.d.). It was not possible to extract details on the specific RT-PCR or antibody-based techniques or platforms used across the included studies due to reporting gaps. Different platforms have varying sensitivity and specificity to detect SARS-CoV-2 infection. In addition, testing for acute infection requires swabbing of the mucosal epithelium, which may be disrupted in current smokers, potentially altering the sensitivity of assays (Lusignan et al., 2020).

\section{Hospitalisation with COVID-19}

i) Reasons for hospitalisation vary by country and time in the pandemic. For example, early cases may have been hospitalised for isolation and quarantine reasons and not due to medical necessity. It is plausible this may have skewed early data towards less severe cases. In addition, the observed association between former smoking and greater disease severity may be explained by collider bias (Griffith et al., 2020), where conditioning on a collider (e.g. testing or hospitalisation) by design or analysis may introduce a spurious association between current or former smoking (a potential cause of testing or hospitalisation) and SARS-CoV-2 infection/adverse outcomes from COVID-19 (potentially exacerbated by smoking) (Murray, 2020).

ii) The majority of included studies relied on EHRs as the source of information on smoking status. Research shows large discrepancies between EHRs and actual behaviour (Polubriaginof et al., 2018). Known failings of EHRs include implausible longitudinal changes, such as former smokers being recorded as never smokers at subsequent hospital visits (Polubriaginof et al., 2018). Misreporting on the part of the patient (perhaps due to perceived stigma) has also been observed, with biochemical measures showing higher rates of smoking compared with self-report in hospitalised patients in the US (Benowitz et al., 2009). It is hence possible that under-reporting of current and former smoking status in hospitals occurred across the included studies.

iii) The majority of included studies were conducted in hospital settings. It is plausible that a non-trivial proportion of patients were infected with SARS-CoV-2 while being an inpatient for a different medical reason. If so, this may have biased the hospitalised populations towards older and more frail groups, who are less likely to be smokers (Mangera et al., 2017).

iv) Individuals with severe COVID-19 symptoms may have stopped smoking immediately before admission to hospital and may therefore not have been recorded as current smokers (i.e. reverse causality).

6. COVID-19 disease severity and death

i) Given lack of knowledge of the disease progression and long-term outcomes of COVID-19, it is unclear whether studies conducted thus far in the pandemic have monitored patients for a sufficient time period to report complete survival outcomes or whether they are subject to early censoring. Adding to this, COVID-19 related mortality has been differentially defined across countries and epidemic phases. For example, in some UK reporting, death within 28 days 
of a COVID-19 diagnosis is required for attributing the cause of death to the virus. However, according to the UK Office for National Statistics, COVID-19 deaths are recorded only if this was stated on the death certificate.

ii) If there is a protective effect of nicotine on COVID-19 disease outcomes, abrupt nicotine withdrawal upon hospitalisation may lead to worse disease outcomes including death (Farsalinos, Niaura, et al., 2020).

iii) During periods of heightened demand of limited healthcare resources, current and former smokers with extensive comorbidities may have reduced priority for intensive care admission, thus leading to higher in-hospital mortality.

iv) COVID-19 outcomes are currently limited to in-hospital death or survival to discharge. This binary outcome does not capture potential long-term morbidity attributed to COVID-19, such as stroke, amputation or acute cardiac events, which may be moderated by smoking status. 
i. Infection following exposure depends on poorly understood individual differences (e.g. genetic susceptibility, immunocompetence)

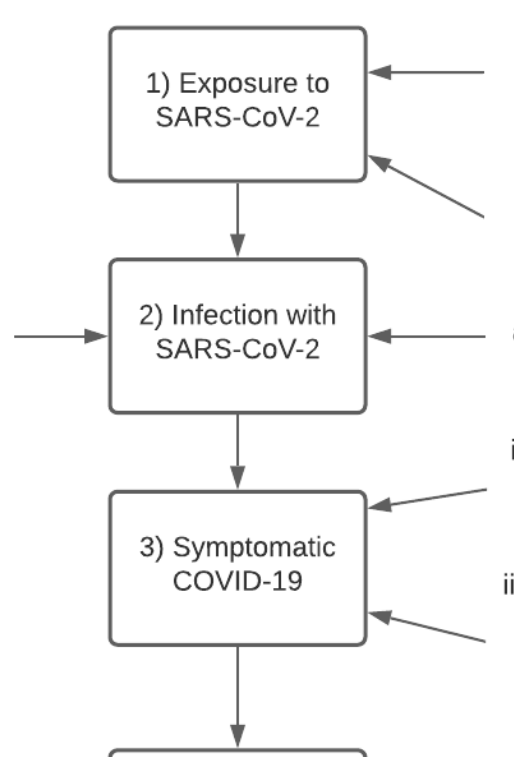

i. Differential access to health care (inequities limiting access to testing or healthcare services)

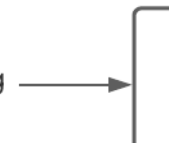

4) Testing positive for SARS-CoV-2

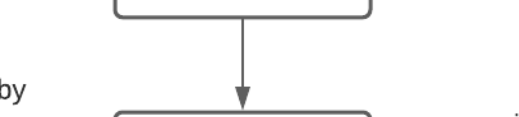

i. Reasons for hospitalisation vary by country and time in the pandemic (e.g. hospitalisation for isolation and quarantine reasons)

iii. Hospital acquired infections

i. It is unclear whether studies have monitored patients for a sufficient period of time to report complete survival outcomes. Mortality from COVID-19 has also been differentially defined across countries and studies i. Exposure to the virus is heterogeneous due to differential contact matrices by age, sex and socioeconomic position, which are associated with smoking

ii. Probability of exposure to infected individuals depends on local prevalence, which is time-varying

ii. Heated and humidified air may act to disrupt the ability of the virus to persist in the airway mucosa of smokers

i. Asymptomatic infection, particularly among younger individuals, may limit testing. Age is associated with smoking status

ii. Symptoms consistent with COVID-19 such as cough or change in smell or taste may be more common in current or former smokers, thus increasing numbers presenting for testing

ii. Sensitivity of tests may differ in current smokers and different techniques and platforms used throughout the pandemic have varying sensitivity and specificity

ii. Misreporting or poor recording of smoking status

iv. Reverse causality (e.g. current smokers quitting immediately prior to hospitalisation)

ii. Acute nicotine withdrawal may lead to increased disease severity in hospitalised current smokers

iii. Current and former smokers may have increased comorbidities compared with never smokers.

Intensive care escalation (as judged by healthcare professionals in discussion with patients) may not be in their best interest.

iv. COVID-19 outcomes are currently limited to death or recovery, with little attention given to long-term morbidity

Figure 11. A schematic of some of the interpretation issues for the association of smoking status and COVID-19 infection, hospitalisation, disease severity and mortality. Numbers refer to the issues listed in-text in the above section. Issues presented on the right-hand side relate directly to smoking status. 
This living rapid evidence review was limited by having a single reviewer extracting data with a second independently verifying the data extracted to minimise errors, restricting the search to one electronic database and one pre-print server and by not including at least three large population surveys due to their reliance on self-reported suspected or confirmed SARS-CoV-2 infection (which means they do not meet our eligibility criteria) (Bowyer et al., 2020; Hopkinson et al., 2020; Jackson et al., 2020). We also did not include a large, UK-based, representative seroprevalence study (Ward et al., 2020) in our meta-analyses as the odds of testing positive in former smokers was not reported. However, the odds of infection for current smokers $(\mathrm{OR}=0.64,95 \% \mathrm{Cl}=0.58-0.71)$ was in concordance with the pooled estimate in our meta-analysis. Population surveys - particularly with linked data on confirmed infection or antibodies - will be included in future review versions to help mitigate some of the limitations of healthcare based observational studies. The comparisons of current and former smoking prevalence in the included studies with national prevalence estimates did not adjust observed prevalence for the demographic profile of those tested/admitted to hospital. Other reviews focused on this comparison have applied adjustments for sex and age, and continue to find lower than expected prevalence - notwithstanding the issues complicating interpretation described above (Farsalinos, Barbouni, et al., 2020).

Implications for research, policy and practice

Further scientific research is needed to resolve the mixed findings summarised in our review. First, clinical trials of the posited therapeutic effect of nicotine could have important implications both for smokers and for improved understanding of how the SARS-CoV-2 virus causes disease in humans. Such trials should focus on medicinal nicotine (as smoked tobacco is a dirty delivery mechanism that could mask beneficial effects) and potentially differentiate between different modes of delivery (i.e. inhaled vs. ingested) since this can affect pharmacokinetics (Shahab et al., 2013) and potential therapeutic effects. A second research priority would be a large, representative (randomly sampled) population survey with a validated assessment of smoking status which distinguishes between recent and long-term ex-smokers - ideally biochemically verified - and assesses seroprevalence and links to health records.

In the meantime, public-facing messages about the possible protective effect of smoking or nicotine are premature. In our view, until there is further research, the quality of the evidence does not justify the huge risk associated with a message likely to reach millions of people that a lethal activity, such as smoking, may protect against COVID-19. It continues to be appropriate to recommend smoking cessation and emphasise the role of alternative nicotine products to support smokers to stop as part of public health efforts during COVID-19. At the very least, smoking cessation reduces acute risks from cardiovascular disease and could reduce demands on the healthcare system (Stead et al., 2013). GPs and other healthcare providers can play a crucial role - brief, high-quality and free online training is available at National Centre for Smoking Cessation and Training.

\section{Conclusion}

Across 405 studies, recorded current but not past smoking prevalence was generally lower than national prevalence 
estimates. Current smokers were at reduced risk of testing positive for SARS-CoV-2 and former smokers were at increased risk of hospitalisation, disease severity and mortality compared with never smokers.

\section{Acknowledgements}

An original short review for the Royal College of Physicians was converted to an extended living review after a request by Martin Dockrell, Tobacco Control Lead, Public Health England. All scientific decisions were made by the authors independently of funders and external organisations. The authors would like to thank Rosemary Koper for her assistance in running the electronic searches and data extraction up until v7, and all authors who responded to requests for additional data.

\section{Declaration of conflicts of interest}

DS and OP have no conflicts of interest to declare. LS has received a research grant and honoraria for a talk and travel expenses from manufacturers of smoking cessation medications (Pfizer and Johnson \& Johnson). JB has received unrestricted research funding to study smoking cessation from companies who manufacture smoking cessation medications. All authors declare no financial links with tobacco companies or e-cigarette manufacturers or their representatives.

\section{Funding statement}

DS is supported by a PhD studentship from the UK Biotechnology and Biological Sciences Research Council [BB/M009513/1]. OP receives salary support from Cancer Research UK (C1417/A22962). JB, LS, \& OP are members of SPECTRUM, a UK Prevention Research Partnership Consortium (MR/S037519/1). UKPRP is an initiative funded by the UK Research and Innovation Councils, the Department of Health and Social Care (England) and the UK devolved administrations, and leading health research charities.

\section{Review versions}

The most up-to-date review version is available here

Previous review versions

- Version 1

- Version 2

- Version 3

- Version 4 
- Version 5

- Version 6

- Version 7

- Version 8

- Version 9

- Version 10

\section{Data availability}

All data contributing to the current and future review versions are available here

All code required to reproduce the current and future analyses are available here

\section{References}

Abadom TR, Smith AD, Tempia S, et al. (2016) Risk factors associated with hospitalisation for influenza-associated severe acute respiratory illness in South Africa: A case-population study. Vaccine 34(46): 5649-5655. DOI:

\subsection{6/j.vaccine.2016.09.011.}

Abajo FJ de, Rodríguez-Miguel A, Rodríguez-Martín S, et al. (2021) IN-HOSPITAL CONTINUATION WITH ANGIOTENSIN RECEPTOR BLOCKERS IS ASSOCIATED WITH A LOWER MORTALITY RATE THAN CONTINUATION WITH ANGIOTENSIN CONVERTING ENZYME INHIBITORS IN COVID-19 PATIENTS: A RETROSPECTIVE COHORT STUDY. DOI: 10.1101/2021.02.01.21250853.

Abolghasemi S, Mardani M, Sali S, et al. (2020) COVID-19 and kidney transplant recipients. Transplant Infectious Disease 22(6). DOI: $10.1111 /$ tid.13413.

Ader F, Peiffer-Smadja N, Poissy J, et al. (2021) Antiviral drugs in hospitalized patients with COVID-19 - the DisCoVeRy

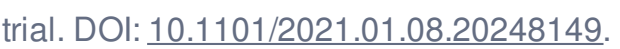

Adrish M, Chilimuri S, Mantri N, et al. (2020) Association of smoking status with outcomes in hospitalised patients with COVID-19. BMJ Open Respiratory Research 7(1): e000716. DOI: 10.1136/bmjresp-2020-000716.

Aksu K, Naziroğlu T and Özkan P (2020) Factors determining COVID-19 pneumonia severity in a country with routine BCG vaccination. Clinical \& Experimental Immunology 202(2): 220-225. DOI: 10.1111/cei.13507.

Alguwaihes AM, Al-Sofiani ME, Megdad M, et al. (2020) Diabetes and Covid-19 among hospitalized patients in Saudi Arabia: A single-centre retrospective study. Cardiovascular Diabetology 19(1): 205. DOI: 10.1186/s12933-020-01184-4. Alharthy A, Aletreby W, Faqihi F, et al. (2020) Clinical Characteristics and Predictors of 28-Day Mortality in 352 Critically III Patients with COVID-19: A Retrospective Study: Journal of Epidemiology and Global Health. DOI:

10.2991/jegh.k.200928.001.

Al-Hindawi A, Sokhi J, Cuddihy J, et al. (2020) COVID-19 in London, a Case Series Demonstrating Late Improvement in Survivors. medRxiv: 2020.05.16.20103853. DOI: 10.1101/2020.05.16.20103853.

Alizadehsani R, Sani ZA, Behjati M, et al. (2020) Risk Factors Prediction, Clinical Outcomes, and Mortality of COVID-19 
Patients. medRxiv: 2020.07.07.20148569. DOI: 10.1101/2020.07.07.20148569.

Alkurt G, Murt A, Aydin Z, et al. (2020) Seroprevalence of Coronavirus Disease 2019 (COVID-19) Among Health Care Workers from Three Pandemic Hospitals of Turkey. medRxiv: 2020.08.19.20178095. DOI: 10.1101/2020.08.19.20178095. Allenbach Y, Saadoun D, Maalouf G, et al. (2020) Development of a multivariate prediction model of intensive care unit transfer or death: A French prospective cohort study of hospitalized COVID-19 patients. PLOS ONE Moreira J (ed.) 15(10): e0240711. DOI: 10.1371/journal.pone.0240711.

Almazeedi S, Al-Youha S, Jamal MH, et al. (2020) Characteristics, risk factors and outcomes among the first consecutive 1096 patients diagnosed with COVID-19 in Kuwait. EClinicalMedicine 24: 100448. DOI: 10.1016/j.eclinm.2020.100448. Almirall J, González CA, Balanzó X, et al. (1999) Proportion of community-acquired pneumonia cases attributable to tobacco smoking. Chest 116: 375-9. DOI: 10.1378/chest.116.2.375.

Alqahtani JS, Oyelade T, Aldhahir AM, et al. (2020) Prevalence, Severity and Mortality associated with COPD and Smoking in patients with COVID-19: A Rapid Systematic Review and Meta-Analysis. medRxiv: 2020.03.25.20043745. DOI: $10.1101 / 2020.03 .25 .20043745$.

Alshami AA, Alattas RA, Anan HF, et al. (2020) Silent Disease and Loss of Taste and Smell are Common Manifestations of SARS-COV-2 Infection in a Quarantine Facility: First report from Saudi Arabia. medRxiv: 2020.05.13.20100222. DOI: $10.1101 / 2020.05 .13 .20100222$.

Altamimi H, Alahmad Y, Khazal F, et al. (2020) The Outcome of COVID-19 Patients with Acute Myocardial Infarction. medRxiv: 2020.07.21.20156349. DOI: 10.1101/2020.07.21.20156349.

Altibi AM, Bhargava P, Liaqat H, et al. (2020) Comparative Clinical Outcomes and Mortality in Prisoner and Non-Prisoner Populations Hospitalized with COVID-19: A Cohort from Michigan. medRxiv: 2020.08.08.20170787. DOI:

10.1101/2020.08.08.20170787.

Andler R (2019) BAISSE DE LA PRÉVALENCE DU TABAGISME QUOTIDIEN PARMI LES ADULTES : RÉSULTATS DU BAROMĖTRE DE SANTÉ PUBLIQUE FRANCE 2018 / REDUCTION OF DAILY SMOKING RATE AMONG ADULTS: RESULTS FROM THE 2018 SANTÉ PUBLIQUE FRANCE HEALTH BAROMETER.: 7.

Antonio-Villa NE, Bello-Chavolla OY, Vargas-Vazquez A, et al. (2020) Health-care workers with COVID-19 living in Mexico City: Clinical characterization and related outcomes. medRxiv: 2020.07.02.20145169. DOl:

10.1101/2020.07.02.20145169.

Apea VJ, Wan YI, Dhairyawan R, et al. (2021) Ethnicity and outcomes in patients hospitalised with COVID-19 infection in East London: An observational cohort study. BMJ open 11. DOI: 10.1136/bmjopen-2020-042140.

Argenziano MG, Bruce SL, Slater CL, et al. (2020) Characterization and clinical course of 1000 patients with coronavirus disease 2019 in New York: Retrospective case series. BMJ: m1996. DOI: 10.1136/bmj.m1996.

Ariza BE, Torres YX, Salgado D, et al. (2020) Seroprevalence and seroconversion rates to SARS-CoV-2 in interns, residents, and medical doctors in a University Hospital in Bogota, Colombia. medRxiv: 2020.09.15.20195313. DOI: 10.1101/2020.09.15.20195313.

Arleo T, Tong D, Shabto J, et al. (2020) Clinical Course and Outcomes of coronavirus disease 2019 (COVID-19) in Rheumatic Disease Patients on Immunosuppression: A case Cohort Study at a Single Center with a Significantly Diverse Population. preprint, October. Epidemiology. DOI: 10.1101/2020.10.26.20219154. 
Auvinen R, Nohynek H, Syrjänen R, et al. (2020) Comparison of the clinical characteristics and outcomes of hospitalized adult COVID-19 and influenza patients: A prospective observational study. medRxiv: 2020.06.29.20140632. DOI: 10.1101/2020.06.29.20140632.

Ayoubkhani D, Khunti K, Nafilyan V, et al. (2021) Epidemiology of post-COVID syndrome following hospitalisation with coronavirus: A retrospective cohort study. DOI: $10.1101 / 2021.01 .15 .21249885$.

Barasa S, Kiage-Mokaya J, Cruz-Madrid K, et al. (2020) Smoking increases the risk of COVID-19 positivity, while Neversmoking reduces the risk. preprint, December. Respiratory Medicine. DOI: 10.1101/2020.11.30.20241380.

Basse C, Diakite S, Servois V, et al. (2020) Characteristics and outcome of SARS-CoV-2 infection in cancer patients. medRxiv: 2020.05.14.20101576. DOI: 10.1101/2020.05.14.20101576.

Batty GD, Deary I, Luciano M, et al. (2020) Psychosocial factors and hospitalisations for COVID-19: Prospective cohort study of the general population. medRxiv: 2020.05.29.20100735. DOI: 10.1101/2020.05.29.20100735.

Bellan M, Patti G, Hayden E, et al. (2020) Fatality rate and predictors of mortality in an Italian cohort of hospitalized COVID19 patients. Scientific Reports 10(1): 20731. DOI: 10.1038/s41598-020-77698-4.

Bello-Chavolla OY, Bahena-López JP, Antonio-Villa NE, et al. (2020) Predicting Mortality Due to SARS-CoV-2: A Mechanistic Score Relating Obesity and Diabetes to COVID-19 Outcomes in Mexico. The Journal of Clinical Endocrinology \& Metabolism 105(8). DOI: 10.1210/clinem/dgaa346.

Bello-Chavolla OY, Antonio-Villa NE, Vargas-Vázquez A, et al. (2020) Profiling pre-symptomatic and asymptomatic cases with confirmed SARS-CoV-2 infection in Mexico City. medRxiv: 2020.07.02.20145516. DOI:

10.1101/2020.07.02.20145516.

Benaim AR, Sobel JA, Almog R, et al. (2020) At the dawn of winter: Comparing COVID-19 and influenza presentation and trajectory. preprint, November. Infectious Diseases (except HIV/AIDS). DOI: 10.1101/2020.11.19.20235077.

Benowitz NL, Schultz KE, Haller CA, et al. (2009) Prevalence of smoking assessed biochemically in an urban public hospital: A rationale for routine cotinine screening. American Journal of Epidemiology 170(7): 885-891. DOI:

10.1093/aje/kwp215.

Berlin I, Thomas D, Le Faou A-L, et al. (2020) COVID-19 and Smoking. Nicotine \& Tobacco Research. DOI: 10.1093/NTR/NTAA059.

Bermejo-Martin JF, González-Rivera M, Almansa R, et al. (2020) Viral RNA load in plasma is associated with critical illness and a dysregulated host response in COVID-19. Critical Care 24(1): 691. DOI: 10.1186/s13054-020-03398-0. Bernaola N, Mena R, Bernaola A, et al. (2020) Observational Study of the Efficiency of Treatments in Patients Hospitalized with Covid-19 in Madrid. medRxiv: 2020.07.17.20155960. DOI: 10.1101/2020.07.17.20155960.

Berumen J, Schmulson M, Alegre J, et al. (2020) Risk of infection and hospitalization by Covid-19 in Mexico: A casecontrol study. medRxiv: 2020.05.24.20104414. DOI: 10.1101/2020.05.24.20104414.

Best JH, Mohan SV, Kong AM, et al. (2020) Baseline Demographics and Clinical Characteristics Among 3471 US Patients Hospitalized with COVID-19 and Pulmonary Involvement: A Retrospective Study. Advances in Therapy 37(12): 49814995. DOI: $10.1007 / \mathrm{s} 12325-020-01510-y$.

Bian H, Zheng Z-H, Wei D, et al. (2020) Meplazumab treats COVID-19 pneumonia: An open-labelled, concurrent controlled add-on clinical trial. medRxiv: 2020.03.21.20040691. DOI: 10.1101/2020.03.21.20040691. 
Bisso IC, Huespe I, Lockhart C, et al. (2020) Clinical characteristics of critically ill patients with COVID-19. preprint, December. Intensive Care; Critical Care Medicine. DOI: 10.1101/2020.12.09.20246413.

Borobia AM, Carcas AJ, Arnalich F, et al. (2020) A Cohort of Patients with COVID-19 in a Major Teaching Hospital in Europe. Journal of Clinical Medicine 9(6): 1733. DOI: 10.3390/jcm9061733.

Boulware DR, Pullen MF, Bangdiwala AS, et al. (2020) A Randomized Trial of Hydroxychloroquine as Postexposure Prophylaxis for Covid-19. New England Journal of Medicine. DOI: 10.1056/NEJMoa2016638.

Bowyer RCE, Varsavsky T, Sudre CH, et al. (2020) Geo-social gradients in predicted COVID-19 prevalence and severity in Great Britain: Results from 2,266,235 users of the COVID-19 Symptoms Tracker app. DOI:

\subsection{1/2020.04.23.20076521.}

Boyd S and Martin-Loeches I (2021) The incidence of venous thromboembolism in critically ill patients with COVID-19 compared with critically ill non-COVID patients. Ir J Med Sci NA. DOI: 10.1007/s11845-020-02503-0.

Brake SJ, Barnsley K, Lu W, et al. (2020) Smoking Upregulates Angiotensin-Converting Enzyme-2 Receptor: A Potential Adhesion Site for Novel Coronavirus SARS-CoV-2 (Covid-19). J Clin Med 9, Page 841: 841. DOI: 10.3390/jcm9030841. Brown J (2020) The Smoking Toolkit Study, 2020. Available at: https://docs.google.com/presentation/d/e/2PACX-

\section{1vQmMeD0wPM7iEawo39m2QZnVBOo2e8YIQPjm2R0OgYBvKhBbYqnarSn5vxyk3-56w/embed?} start=false \&loop=false \&delayms $=3000 \&$ usp $=$ embed_facebook.

Buitrago-Garcia D, Egli-Gany D, Counotte MJ, et al. (2020) Occurrence and transmission potential of asymptomatic and presymptomatic SARS-CoV-2 infections: A living systematic review and meta-analysis. PLOS Medicine 17(9): e1003346. DOI: 10.1371 /journal.pmed.1003346.

Burrell AJ, Pellegrini B, Salimi F, et al. (2020) Outcomes of COVID-19 patients admitted to Australian intensive care units during the early phase of the pandemic. The Medical Journal of Australia: 1. DOI: $10.5694 / \mathrm{mja2} .50883$.

Bürkner P-C (2018) Advanced Bayesian Multilevel Modeling with the R Package brms. The R Journal 10(1): 395. DOI: 10.32614/RJ-2018-017.

Cadegiani FA, McCoy J, Wambier CG, et al. (2020) 5-Alpha-Reductase Inhibitors Reduce Remission Time of COVID-19: Results From a Randomized Double Blind Placebo Controlled Interventional Trial in 130 SARS-CoV-2 Positive Men. preprint, November. Infectious Diseases (except HIV/AIDS). DOI: 10.1101/2020.11.16.20232512.

Caglar A and Kacer I (2021) Anxiety levels in patients admitted to the emergency department with myocardial infarction or COVID-19 pneumonia. PSYCHOL HEALTH MED NA: 1-9. DOI: 10.1080/13548506.2021.1876893.

Cai G (2020) Bulk and Single-Cell Transcriptomics Identify Tobacco-Use Disparity in Lung Gene Expression of ACE2, the Receptor of 2019-nCov. DOI: 10.20944/PREPRINTS202002.0051.V3.

Cai H, Yang L, Lu Y, et al. (2021) High body mass index is a significant risk factor for the progression and prognosis of imported COVID-19: A multicenter, retrospective cohort study. BMC Infect Dis 21. DOI: 10.1186/s12879-021-05818-0. Caliskan T and Saylan B (2020) Smoking and comorbidities are associated with COVID-19 severity and mortality in 565 patients treated in Turkey: A retrospective observational study. Rev Assoc Med Bras 66: 1679-1684. DOI: 10.1590/1806$\underline{9282.66 .12 .1679 .}$.

Carrat F, Lamballerie X de, Rahib D, et al. (2020) Seroprevalence of SARS-CoV-2 among adults in three regions of France following the lockdown and associated risk factors: A multicohort study. medRxiv: 2020.09.16.20195693. DOI: 


\section{$\underline{10.1101 / 2020.09 .16 .20195693}$.}

Carrillo-Vega MF, Salinas-Escudero G, Garcia-Peña C, et al. (2020) Early estimation of the risk factors for hospitalisation and mortality by COVID-19 in Mexico. medRxiv: 2020.05.11.20098145. DOI: 10.1101/2020.05.11.20098145.

CDCMMWR (2020) Preliminary Estimates of the Prevalence of Selected Underlying Health Conditions Among Patients with Coronavirus Disease 2019 - United States, February 12-March 28, 2020. MMWR. Morbidity and Mortality Weekly Report 69. DOI: 10.15585/mmwr.mm6913e2.

Cen Y, Chen X, Shen Y, et al. (2020) Risk factors for disease progression in patients with mild to moderate coronavirus disease 2019—a multi-centre observational study. Clinical Microbiology and Infection: S1198743X20303414. DOI: 10.1016/j.cmi.2020.05.041.

Chand S, Kapoor S, Orsi D, et al. (2020) COVID-19-Associated Critical IIIness-Report of the First 300 Patients Admitted to Intensive Care Units at a New York City Medical Center: Journal of Intensive Care Medicine. DOI:

\section{$10.1177 / 0885066620946692$.}

Chaudhary A, Singh UN, Paudel P, et al. (2020) Characteristics and outcomes of hospitalized adults with COVID-19 in Nepal: A multicenter, prospective cohort study. medRxiv: 2020.10.03.20206128. DOI: 10.1101/2020.10.03.20206128. Chaudhry F, Bulka H, Rathnam AS, et al. (2020) COVID-19 in Multiple Sclerosis Patients and Risk Factors for Severe Infection. medRxiv: 2020.05.27.20114827. DOI: 10.1101/2020.05.27.20114827.

Chen C, Jiang J, Xu X, et al. (2020) Dynamic liver function indexes monitoring and clinical characteristics in three types of COVID-19 patients. medRxiv: 2020.05.13.20099614. DOI: 10.1101/2020.05.13.20099614.

Chen H, Varatharajah Y, Ramirez SS de, et al. (2020) A Retrospective Longitudinal Study of COVID-19 as Seen by a Large Urban Hospital in Chicago. preprint, November. Infectious Diseases (except HIV/AIDS). DOI: 10.1101/2020.11.29.20240606.

Chen L, Yu J, He W, et al. (2020) Risk factors for death in 1859 subjects with COVID-19. Leukemia: 1-11. DOI: 10.1038/s41375-020-0911-0.

Chen T, Wu D, Chen H, et al. (2020) Clinical characteristics of 113 deceased patients with coronavirus disease 2019: Retrospective study. BMJ 368. DOI: 10.1136/bmj.m1091.

Cho ER, Slutsky AS and Jha P (2020) Smoking and the risk of COVID-19 infection in the UK Biobank Prospective Study. medRxiv: 2020.05.05.20092445. DOI: 10.1101/2020.05.05.20092445.

Chudasama YV, Zaccardi F, Gillies CL, et al. (2020) Patterns of Multimorbidity and Risk of Severe SARS-CoV-2 Infection: An observational study in the U.K. preprint, October. Epidemiology. DOI: 10.1101/2020.10.21.20216721.

Clavario P, De Marzo V, Lotti R, et al. (2020) Assessment of functional capacity with cardiopulmonary exercise testing in non-severe COVID-19 patients at three months follow-up. preprint, November. Cardiovascular Medicine. DOI:

$10.1101 / 2020.11 .15 .20231985$.

CMMID COVID-19 working group, Jarvis Cl, Van Zandvoort K, et al. (2020) Quantifying the impact of physical distance measures on the transmission of COVID-19 in the UK. BMC Medicine 18(1): 124. DOI: 10.1186/s12916-020-01597-8. Collard D, Nurmohamed NS, Kaiser Y, et al. (2020) Cardiovascular risk factors are independently associated with COVID19 mortality: A prospective cohort study. preprint, October. Infectious Diseases (except HIV/AIDS). DOI:

$10.1101 / 2020.10 .01 .20205229$. 
Concha-Mejia A and Rincon-Sanchez RA (2020) CCOFEE-GI Study: Colombian COVID19 First Experience in Gastroentrology. Characterization of digestive manifestations in patients diagnosed with COVID-19 at a highly complex institution in Bogota D.C., Colombia. medRxiv: 2020.07.24.20161604. DOI: 10.1101/2020.07.24.20161604.

Conti C, Marco A de, Mastromarino P, et al. (1999) Antiviral Effect of Hyperthermic Treatment in Rhinovirus Infection. Antimicrobial Agents and Chemotherapy 43(4): 822-829. Available at: https://www.ncbi.nlm.nih.gov/pmc/articles/PMC89212/ (accessed 1 October 2020). Covid-19 in pregnancy, Vousden N, Bunch K, et al. (2021) The incidence, characteristics and outcomes of pregnant women hospitalized with symptomatic and asymptomatic SARS-CoV-2 infection in the UK from March to September 2020: A national cohort study using the UK Obstetric Surveillance System (UKOSS). DOI: 10.1101/2021.01.04.21249195. Crooks CJ, West J, Fogarty A, et al. (2020) Predicting the need for escalation of care or death from repeated daily clinical observations and laboratory results in patients with SARS-CoV-2 during 2020: A retrospective population-based cohort study from the United Kingdom. DOI: $10.1101 / 2020.12 .14 .20248181$.

Crovetto F, Crispi F, Llurba E, et al. (2020) SEROPREVALENCE AND CLINICAL SPECTRUM OF SARS-CoV-2 INFECTION IN THE FIRST VERSUS THIRD TRIMESTER OF PREGNANCY. medRxiv: 2020.06.17.20134098. DOI: 10.1101/2020.06.17.20134098.

Cummins L, Ebyarimpa I, Cheetham N, et al. (2021) Factors associated with COVID-19 related hospitalisation, critical care admission and mortality using linked primary and secondary care data. DOI: 10.1101/2021.01.19.20241844.

Dai M, Tao L, Chen Z, et al. (2020) Influence of Cigarettes and Alcohol on the Severity and Death of COVID-19: A Multicenter Retrospective Study in Wuhan, China. Front Physiol 11. DOI: 10.3389/fphys.2020.588553.

Dashti H, Roche E, Bates D, et al. (2020) SARS2 simplified scores to estimate risk of hospitalization and death among patients with COVID-19. DOI: 10.1101/2020.09.11.20190520.

Denholm JT, Gordon CL, Johnson PD, et al. (2010) Hospitalised adult patients with pandemic (H1N1) 2009 influenza in Melbourne, Australia. Medical Journal of Australia 192(2): 84-86. DOI: 10.5694/j.1326-5377.2010.tb03424.x.

Didikoglu A, Maharani A, Pendleton N, et al. (2021) Early life factors and COVID-19 infection in England: A prospective analysis of UK Biobank participants. Early Hum Dev 155. DOI: 10.1016/j.earlhumdev.2021.105326.

Díez-Manglano J, Marquínez MNS, García AÁ, et al. (2020) Healthcare workers hospitalized due to COVID-19 have no higher risk of death than general population. Data from the Spanish SEMI-COVID-19 Registry. preprint, November. Infectious Diseases (except HIV/AIDS). DOI: 10.1101/2020.11.23.20236810.

Docherty AB, Harrison EM, Green CA, et al. (2020) Features of 20133 UK patients in hospital with covid-19 using the ISARIC WHO Clinical Characterisation Protocol: Prospective observational cohort study. BMJ 369. DOI:

10.1136/bmj.m1985.

Dong X, Cao Y-y, Lu X-x, et al. (2020) Eleven faces of coronavirus disease 2019. Allergy 75(7): 1699-1709. DOI: 10.1111/all.14289.

Duan L, Zhang S, Guo M, et al. (2020) Epidemiological and clinical characteristics in patients with SARS-CoV-2 antibody negative probable COVID-19 in Wuhan. medRxiv: 2020.06.18.20134619. DOI: 10.1101/2020.06.18.20134619. Dupraz J, Butty A, Duperrex O, et al. (2020) Prevalence of SARS-CoV-2 in household members and other close contacts of COVID-19 cases: A serologic study in canton of Vaud, Switzerland. preprint, November. Epidemiology. DOI: 


\section{$10.1101 / 2020.11 .27 .20239244$}

Dye JA and Adler KB (1994) Occasional review Effects of cigarette smoke on epithelial cells of the respiratory tract. Thorax 49: 825-834. DOI: 10.1136/thx.49.8.825.

Ebinger J, Botwin GJ, Albert CM, et al. (2020) SARS-CoV-2 Seroprevalence Across a Diverse Cohort of Healthcare Workers. medRxiv: 2020.07.31.20163055. DOI: 10.1101/2020.07.31.20163055.

Ebrahimian S, Homayounieh F, Rockenbach MABC, et al. (2021) Artificial intelligence matches subjective severity assessment of pneumonia for prediction of patient outcome and need for mechanical ventilation: A cohort study. Sci. rep 11. DOI: $10.1038 / \mathrm{s} 41598-020-79470-0$.

Eiros R, Barreiro-Perez M, Martin-Garcia A, et al. (2020) Pericarditis and myocarditis long after SARS-CoV-2 infection: A cross-sectional descriptive study in health-care workers. medRxiv: 2020.07.12.20151316. DOI:

10.1101/2020.07.12.20151316.

Elezkurtaj S, Greuel S, Ihlow J, et al. (2020) Causes of Death and Comorbidities in Patients with COVID-19. medRxiv: 2020.06.15.20131540. DOI: $10.1101 / 2020.06 .15 .20131540$.

Elliott JH, Turner T, Clavisi O, et al. (2014) Living Systematic Reviews: An Emerging Opportunity to Narrow the EvidencePractice Gap. PLoS Medicine 11(2). DOI: 10.1371/journal.pmed.1001603.

Elmunzer BJ, Spitzer RL, Foster LD, et al. (2020) Digestive Manifestations in Patients Hospitalized with COVID-19. medRxiv: 2020.07.07.20143024. DOI: 10.1101/2020.07.07.20143024.

El-Solh AA, Meduri UG, Lawson Y, et al. (2020) CLINICAL COURSE AND OUTCOME OF COVID-19 ACUTE RESPIRATORY DISTRESS SYNDROME: DATA FROM A NATIONAL REPOSITORY. medRXiv: 2020.10.16.20214130. DOI: $10.1101 / 2020.10 .16 .20214130$.

Emami A, Javanmardi F, Pirbonyeh N, et al. (2020) Prevalence of Underlying Diseases in Hospitalized Patients with COVID-19: A Systematic Review and Meta-Analysis. Archives of academic emergency medicine 8(1): e35.

Erber J, Kappler V, Haller B, et al. (2020) Strategies for infection control and prevalence of anti-SARS-CoV-2 IgG in 4,554 employees of a university hospital in Munich, Germany. preprint, October. Epidemiology. DOI:

10.1101/2020.10.04.20206136.

Estiri H, Strasser ZH, Klann JG, et al. (2021) Predicting COVID-19 mortality with electronic medical records. npj digit. med 4. DOI: $10.1038 / \mathrm{s} 41746-021-00383-\mathrm{x}$.

Eugen-Olsen J, Altintas I, Tingleff J, et al. (2020) Low levels of the prognostic biomarker suPAR are predictive of mild outcome in patients with symptoms of COVID-19 - a prospective cohort study. medRxiv: 2020.05.27.20114678. DOI: 10.1101/2020.05.27.20114678.

Fan X, Yin C, Wang J, et al. (2020) Pre-diagnostic circulating concentrations of insulin-like growth factor-1 and risk of COVID-19 mortality: Results from UK Biobank. medRxiv: 2020.07.09.20149369. DOI: 10.1101/2020.07.09.20149369. Farsalinos K, Niaura R, Le Houezec J, et al. (2020) Editorial: Nicotine and SARS-CoV-2: COVID-19 may be a disease of the nicotinic cholinergic system. Toxicology Reports. DOI: 10.1016/j.toxrep.2020.04.012.

Farsalinos K, Barbouni A and Niaura R (2020) Systematic review of the prevalence of current smoking among hospitalized COVID-19 patients in China: Could nicotine be a therapeutic option? Internal and Emergency Medicine. DOI:

\subsection{7/s11739-020-02355-7.}


Favara DM, McAdam K, Cooke A, et al. (2020) SARS-CoV-2 antigen and antibody prevalence among UK staff working with cancer patients during the COVID-19 pandemic. DOI: $10.1101 / 2020.09 .18 .20197590$.

Feldman C and Anderson R (2013) Cigarette smoking and mechanisms of susceptibility to infections of the respiratory tract and other organ systems. J. Infect 67: 169-84. DOI: 10.1016/i.jinf.2013.05.004.

Feng Y, Ling Y, Bai T, et al. (2020) COVID-19 with Different Severities: A Multicenter Study of Clinical Features. American Journal of Respiratory and Critical Care Medicine 201(11): 1380-1388. DOI: 10.1164/rccm.202002-0445OC.

Ferrari BL, Ferreira CG, Menezes M, et al. (2021) Determinants of COVID-19 Mortality in Patients With Cancer From a Community Oncology Practice in Brazil. JCO Glob Oncol 7: 46-55. DOI: 10.1200/G0.20.00444.

Feuth T, Saaresranta T, Karlsson A, et al. (2020) Is sleep apnoea a risk factor for Covid-19? Findings from a retrospective cohort study. medRxiv: 2020.05.14.20098319. DOI: 10.1101/2020.05.14.20098319.

Fillmore NR, La J, Szalat RE, et al. (2020) Prevalence and outcome of Covid-19 infection in cancer patients: A national VA study. medRxiv: 2020.08.21.20177923. DOI: $10.1101 / 2020.08 .21 .20177923$.

Fisman D, Greer AL and Tuite A (2020) Derivation and Validation of Clinical Prediction Rule for COVID-19 Mortality in Ontario, Canada. medRxiv: 2020.06.21.20136929. DOI: 10.1101/2020.06.21.20136929.

Fond G, Pauly V, Orleans V, et al. (2020) Increased in-hospital mortality from COVID-19 in patients with schizophrenia. L'Encéphale: S0013700620301883. DOI: 10.1016/j.encep.2020.07.003.

Fontanet A, Tondeur L, Madec Y, et al. (2020) Cluster of COVID-19 in northern France: A retrospective closed cohort study. medRxiv: 2020.04.18.20071134. DOI: 10.1101/2020.04.18.20071134.

Fox TA, Troy-Barnes E, Kirkwood AA, et al. (n.d.) Clinical outcomes and risk factors for severe COVID-19 infection in patients with haematological disorders receiving chemo- or immunotherapy. British Journal of Haematology n/a(n/a). DOI: 10.1111/bjh.17027.

Freites D, Leon L, Mucientes A, et al. (2020) Risk factors for hospital admission related to COVID-19 in inflammatory rheumatic diseases. medRxiv: 2020.05.14.20101584. DOI: 10.1101/2020.05.14.20101584.

Fung HF, Martinez L, Alarid-Escudero F, et al. (2020) The household secondary attack rate of SARS-CoV-2: A rapid review. Clinical Infectious Diseases. DOI: 10.1093/cid/ciaa1558.

Galal I, Hussein AAM, Amin MT, et al. (2020) Determinants of Persistent Post COVID-19 symptoms: Value of a Novel COVID-19 symptoms score. DOI: 10.1101/2020.11.11.20230052.

Gallichotte EN, Quicke KM, Sexton NR, et al. (2020) Longitudinal Surveillance for SARS-CoV-2 Among Staff in Six Colorado Long-Term Care Facilities: Epidemiologic, Virologic and Sequence Analysis. medRxiv: 2020.06.08.20125989. DOI: $10.1101 / 2020.06 .08 .20125989$.

Garassino MC, Whisenant JG, Huang L-C, et al. (2020) COVID-19 in patients with thoracic malignancies (TERAVOLT): First results of an international, registry-based, cohort study. The Lancet Oncology 21(7): 914-922. DOI: 10.1016/S14702045(20)30314-4.

Garibaldi BT, Fiksel J, Muschelli J, et al. (2020) Patient trajectories and risk factors for severe outcomes among persons hospitalized for COVID-19 in the Maryland/DC region. medRxiv: 2020.05.24.20111864. DOI:

$10.1101 / 2020.05 .24 .20111864$.

Ge H, Zhu M, Du J, et al. (2020) Cardiac Structural and Functional Characteristics in Patients with Coronavirus Disease 
2019: A Serial Echocardiographic Study. medRxiv: 2020.05.12.20095885. DOI: 10.1101/2020.05.12.20095885.

Gégout petit A, Jeulin H, Legrand K, et al. (2021) Seroprevalence of SARS-CoV-2, symptom profiles and seroneutralization during the first COVID-19 wave in a suburban area, France. DOI: 10.1101/2021.02.10.21250862. Ghinai I, Davis ES, Mayer S, et al. (2020) Risk Factors for Severe Acute Respiratory Syndrome Coronavirus 2 Infection in Homeless Shelters in Chicago, Illinois-March-May, 2020. Open Forum Infectious Diseases 7(11): ofaa477. DOI:

\subsection{3/ofid/ofaa477.}

Giacomelli A, Ridolfo AL, Milazzo L, et al. (2020) 30-day mortality in patients hospitalized with COVID-19 during the first wave of the Italian epidemic: A prospective cohort study. Pharmacological Research 158: 104931. DOI:

\subsection{6/j.phrs.2020.104931.}

Gianfrancesco M, Hyrich KL, Al-Adely S, et al. (2020) Characteristics associated with hospitalisation for COVID-19 in people with rheumatic disease: Data from the COVID-19 Global Rheumatology Alliance physician-reported registry. Annals of the Rheumatic Diseases 79(7): 859-866. DOI: 10.1136/annrheumdis-2020-217871.

Gianfrancesco MA, Leykina LA, Izadi Z, et al. (2020) Race/ethnicity association with COVID-19 outcomes in rheumatic disease: Data from the COVID-19 Global Rheumatology Alliance Physician Registry. Arthritis \& Rheumatology: art.41567. DOI: $10.1002 /$ art.41567.

Giannini S, Passeri G, Tripepi G, et al. (2021) Effectiveness of In-Hospital Cholecalciferol Use on Clinical Outcomes in Comorbid COVID-19 Patients: A Hypothesis-Generating Study. Nutrients 13. DOI: 10.3390/nu13010219.

Giannouchos T, Sussman R, Mier JM, et al. (2020) Characteristics and risk factors for COVID-19 diagnosis and adverse outcomes in Mexico: An analysis of 89,756 laboratory-confirmed COVID-19 cases. medRxiv: 2020.06.04.20122481. DOI: 10.1101/2020.06.04.20122481.

Gil-Agudo A, Rodriguez-Cola M, Jimenez-Velasco I, et al. (2020) Clinical features of coronavirus disease 2019 (COVID$19)$ in a cohort of patients with disability due to spinal cord injury. medRxiv: 2020.04.20.20072918. DOI: 10.1101/2020.04.20.20072918.

Girardeau Y, Gallois Y, De Bonnecaze G, et al. (2020) Confirmed central olfactory system lesions on brain MRI in COVID19 patients with anosmia: A case-series. DOI: 10.1101/2020.07.08.20148692.

Gold JAW, Wong KK, Szablewski CM, et al. (2020) Characteristics and Clinical Outcomes of Adult Patients Hospitalized with COVID-19 - Georgia, March 2020. MMWR. Morbidity and Mortality Weekly Report 69(18): 545-550. DOI:

10.15585/mmwr.mm6918e1.

Gori M, Berzuini C, D’Elia E, et al. (2020) Exposure to Renin-Angiotensin System Inhibitors Is Associated with Reduced Mortality of Older Hypertensive Covid-19 Patients. DOI: 10.1101/2020.12.15.20247999.

Govind R, Freitas DF de, Pritchard MR, et al. (2020) Clozapine treatment and risk of COVID-19. medRxiv. 2020.06.17.20133595. DOI: 10.1101/2020.06.17.20133595.

Goyal P, Choi JJ, Pinheiro LC, et al. (2020) Clinical Characteristics of Covid-19 in New York City. New England Journal of Medicine 382(24): 2372-2374. DOI: 10.1056/NEJMc2010419.

Griffith G, Morris TT, Tudball M, et al. (2020) Collider bias undermines our understanding of COVID-19 disease risk and severity. medRxiv: 2020.05.04.20090506. DOI: 10.1101/2020.05.04.20090506.

Grundy* EJ, Suddek* T, Filippidis FT, et al. (2020) Smoking, SARS-CoV-2 and COVID-19: A review of reviews 
considering implications for public health policy and practice. Tobacco Induced Diseases 18(July). DOI: $10.18332 / \mathrm{tid} / 124788$.

Gu T, Mack JA, Salvatore M, et al. (2020) COVID-19 outcomes, risk factors and associations by race: A comprehensive analysis using electronic health records data in Michigan Medicine. medRxiv: 2020.06.16.20133140. DOI:

10.1101/2020.06.16.20133140.

Guan W-j, Ni Z-y, Hu Y, et al. (2020) Clinical Characteristics of Coronavirus Disease 2019 in China. New England Journal of Medicine. DOI: 10.1056/NEJMoa2002032.

Guan W-j, Liang W-h, Zhao Y, et al. (2020) Comorbidity and its impact on 1590 patients with COVID-19 in China: A nationwide analysis. European Respiratory Journal 55(5). DOI: 10.1183/13993003.00547-2020.

Guo T, Fan Y, Chen M, et al. (2020) Cardiovascular Implications of Fatal Outcomes of Patients With Coronavirus Disease 2019 (COVID-19). JAMA Cardiology 5(7): 811-818. DOI: 10.1001/jamacardio.2020.1017.

Gupta R, Agrawal R, Bukhari Z, et al. (2020) Higher Comorbidities and Early Death is Characteristic of Hospitalized African-American Patients with COVID-19. medRxiv: 2020.07.15.20154906. DOI: 10.1101/2020.07.15.20154906. Hadi YB, Naqvi SFZ, Kupec JT, et al. (2020) Characteristics and outcomes of COVID-19 in patients with HIV: A multicentre research network study. AIDS 34(13): F3-F8. DOI: 10.1097/QAD.0000000000002666.

Hadjadj J, Yatim N, Barnabei L, et al. (2020) Impaired type I interferon activity and exacerbated inflammatory responses in severe Covid-19 patients. medRxiv: 2020.04.19.20068015. DOI: 10.1101/2020.04.19.20068015.

Hamadah H, Alahmad B, Behbehani M, et al. (2020) COVID-19 clinical outcomes and nationality: Results from a Nationwide registry in Kuwait. BMC Public Health 20(1): 1384. DOI: 10.1186/s12889-020-09490-y.

Hamer M, Kivimäki M, Gale CR, et al. (2020) Lifestyle risk factors, inflammatory mechanisms, and COVID-19 hospitalization: A community-based cohort study of 387,109 adults in UK. Brain, Behavior, and Immunity 87: 184-187. DOI: 10.1016/j.bbi.2020.05.059.

Hao S-R, Zhang S-Y, Lian J-S, et al. (2020) Liver Enzyme Elevation in Coronavirus Disease 2019: A Multicenter, Retrospective, Cross-Sectional Study. The American Journal of Gastroenterology. DOI: 10.14309/ajg.0000000000000717. Heili-Frades S (n.d.) COVID-19 Outcomes in 4712 consecutively confirmed SARS-CoV2 cases in the city of Madrid. medRxiv. DOI: $10.1101 / 2020.05 .22 .20109850$.

Hernández-Garduño E (2020) Obesity is the comorbidity more strongly associated for Covid-19 in Mexico. A case-control study. Obesity Research \& Clinical Practice. DOI: 10.1016/j.orcp.2020.06.001.

Hewitt J, Carter B, Vilches-Moraga A, et al. (2020) The effect of frailty on survival in patients with COVID-19 (COPE): A multicentre, European, observational cohort study. The Lancet Public Health 5(8): e444-e451. DOI: 10.1016/S24682667(20)30146-8.

Higuchi T, Nishida T, Iwahashi H, et al. (2020) Early Clinical Factors Predicting the Development of Critical Disease in Japanese Patients with COVID-19: A Single-Center Retrospective, Observational Study. medRxiv: 2020.07.29.20159442. DOI: $10.1101 / 2020.07 .29 .20159442$.

Hippisley-Cox J, Young D, Coupland C, et al. (2020) Risk of severe COVID-19 disease with ACE inhibitors and angiotensin receptor blockers: Cohort study including 8.3 million people. Heart. heartjnl-2020-317393. DOI:

10.1136/heartjnl-2020-317393. 
Ho FK, Celis-Morales CA, Gray SR, et al. (2020) Modifiable and non-modifiable risk factors for COVID-19, and comparison to risk factors for influenza and pneumonia: Results from a UK Biobank prospective cohort study. BMJ open 10. DOI: 10.1136/bmjopen-2020-040402.

Ho KS, Narasimhan B, Sheehan J, et al. (2020) Controversy over Smoking in COVID-19 - A Real World Experience in New York City. J Med Virol NA. DOI: 10.1002/jmv.26738.

Hoertel N, Rico MS, Vernet R, et al. (2020) Association between SSRI Antidepressant Use and Reduced Risk of Intubation or Death in Hospitalized Patients with Coronavirus Disease 2019: A Multicenter Retrospective Observational Study. medRxiv: 2020.07.09.20143339. DOI: 10.1101/2020.07.09.20143339.

Hoertel N, Sánchez M, Vernet R, et al. (2020) Dexamethasone use and Mortality in Hospitalized Patients with Coronavirus Disease 2019: A Multicenter Retrospective Observational Study. preprint, October. Infectious Diseases (except HIV/AIDS). DOI: $10.1101 / 2020.10 .23 .20218172$.

Hoertel N, Rico MS, Vernet R, et al. (2020) Observational Study of Haloperidol in Hospitalized Patients with Covid-19. medRxiv: 2020.07.15.20150490. DOI: 10.1101/2020.07.15.20150490.

Hoffmann M, Kleine-Weber H, Schroeder S, et al. (2020) SARS-CoV-2 Cell Entry Depends on ACE2 and TMPRSS2 and Is Blocked by a Clinically Proven Protease Inhibitor. Cell 181(2): 271-280.e8. DOI: 10.1016/j.cell.2020.02.052. Holman N, Knighton P, Kar P, et al. (2020) Risk factors for COVID-19-related mortality in people with type 1 and type 2 diabetes in England: A population-based cohort study. The Lancet Diabetes \& Endocrinology 0(0). DOI: 10.1016/S22138587(20)30271-0.

Hopkinson NS, Rossi N, Moustafa JE-S, et al. (2020) Current tobacco smoking and risk from COVID-19: Results from a population symptom app in over 2.4 million people. medRxiv: 2020.05.18.20105288. DOI: 10.1101/2020.05.18.20105288. Houlihan CF, Vora N, Byrne T, et al. (2020) Pandemic peak SARS-CoV-2 infection and seroconversion rates in London frontline health-care workers. The Lancet. S0140673620314847. DOI: 10.1016/S0140-6736(20)31484-7.

Hu L, Chen S, Fu Y, et al. (2020) Risk Factors Associated with Clinical Outcomes in 323 COVID-19 Patients in Wuhan, China. medRxiv: 2020.03.25.20037721. DOI: 10.1101/2020.03.25.20037721.

Huang C, Wang Y, Li X, et al. (2020) Clinical features of patients infected with 2019 novel coronavirus in Wuhan, China. The Lancet 395(10223): 497-506. DOI: 10.1016/S0140-6736(20)30183-5.

Huang Y, Yang R, Xu Y, et al. (2020) Clinical characteristics of 36 non-survivors with COVID-19 in Wuhan, China. medRxiv: 2020.02.27.20029009. DOI: 10.1101/2020.02.27.20029009.

Hultcrantz M, Richter J, Rosenbaum C, et al. (2020) COVID-19 infections and outcomes in patients with multiple myeloma in New York City: A cohort study from five academic centers. medRxiv: 2020.06.09.20126516. DOI:

10.1101/2020.06.09.20126516.

Hussein MH, Toraih EA, Attia AS, et al. (2020) Asthma in COVID-19: An extra chain fitting around the neck? medRxiv: 2020.07.13.20153130. DOI: $10.1101 / 2020.07 .13 .20153130$.

Ibarra-Nava I, Flores-Rodriguez KG, Ruiz-Herrera V, et al. (2020) Ethnic disparities in COVID-19 mortality in Mexico: A cross-sectional study based on national data. medRxiv: 2020.08.26.20182543. DOI: $10.1101 / 2020.08 .26 .20182543$. Ibrahim D, Dulipsingh L, Zapatka L, et al. (2020) Factors Associated with Good Patient Outcomes Following Convalescent Plasma in COVID-19: A Prospective Phase II Clinical Trial. medRxiv: 2020.08.27.20183293. DOI: 


\section{$10.1101 / 2020.08 .27 .20183293$}

Iftime S, López-Azcona AF, Vallverdu I, et al. (2020) First and second waves of coronavirus disease-19: A comparative study in hospitalized patients in Reus, Spain. preprint, December. Infectious Diseases (except HIV/AIDS). DOI: 10.1101/2020.12.10.20246959.

Ikitimur H, Uysal BB, Cengiz M, et al. (n.d.) "Determining Host Factors Contributing to Disease Severity in a Family Cluster of 29 Hospitalized SARS-CoV-2 Patients: Could Genetic Factors Be Relevant in the Clinical Course of COVID-19?". Journal of Medical Virology n/a(n/a). DOI: 10.1002/jmv.26106.

Ilic I, Zdravkovic M, Timcic S, et al. (2021) Pneumonia in healthcare workers during a COVID-19 outbreak at a cardiovascular hospitals. International Journal of Infectious Diseases 103: 188-193. DOI: 10.1016/j.jij. 2020.11.156. Incerti D, Rizzo S, Li X, et al. (2020) Risk factors for mortality among hospitalized patients with COVID-19. preprint, September. Infectious Diseases (except HIV/AIDS). DOI: 10.1101/2020.09.22.20196204. International Severe Acute Respiratory and emerging Infections Consortium, Escher M, Hall M, et al. (2020) ISARIC Clinical Data Report 10 February 2021. DOI: 10.1101/2020.07.17.20155218.

Invernizzi A, Torre A, Parrulli S, et al. (2020) Retinal findings in patients with COVID-19: Results from the SERPICO-19 study. EClinicalMedicine 27: 100550. DOI: 10.1016/j.eclinm.2020.100550.

loannou GN, Locke E, Green P, et al. (2020) Risk Factors for Hospitalization, Mechanical Ventilation, or Death Among 10131 US Veterans With SARS-CoV-2 Infection. JAMA Network Open 3(9): e2022310. DOI:

10.1001/jamanetworkopen.2020.22310.

Ip A, Berry DA, Hansen E, et al. (2020) Hydroxychloroquine and Tocilizumab Therapy in COVID-19 Patients - An Observational Study. medRxiv: 2020.05.21.20109207. DOI: 10.1101/2020.05.21.20109207.

Islam MZ, Riaz BK, Islam AS, et al. (2020) Risk factors associated with morbidity and mortality outcomes of COVID-19 patients on the 14th and 28th day of the disease course: A retrospective cohort study in Bangladesh. medRxiv: 2020.08.17.20176586. DOI: 10.1101/2020.08.17.20176586.

Israel A, Schäffer AA, Cicurel A, et al. (2020) Large population study identifies drugs associated with reduced COVID-19 severity. medRxiv: 2020.10.13.20211953. DOI: $10.1101 / 2020.10 .13 .20211953$.

Israel A, Feldhamer I, Lahad A, et al. (2020) Smoking and the risk of COVID-19 in a large observational population study. medRxiv: 2020.06.01.20118877. DOI: 10.1101/2020.06.01.20118877.

Iversen K, Bundgaard H, Hasselbalch RB, et al. (2020) Risk of COVID-19 in health-care workers in Denmark: An observational cohort study. The Lancet Infectious Diseases 0(0). DOI: 10.1016/S1473-3099(20)30589-2. Izquierdo JL, Almonacid C, Gonzalez Y, et al. (2020) The impact of COVID-19 on patients with asthma. medRxiv: 2020.07.24.20161596. DOI: 10.1101/2020.07.24.20161596.

Izzi-Engbeaya C, Distaso W, Amin A, et al. (2020) Severe COVID-19 and Diabetes: A Retrospective Cohort Study from Three London Teaching Hospitals. medRxiv: 2020.08.07.20160275. DOI: 10.1101/2020.08.07.20160275. Jackson SE, Brown J, Shahab L, et al. (2020) COVID-19, smoking and inequalities: A study of 53002 adults in the UK. Tobacco Control: tobaccocontrol-2020-055933. DOI: 10.1136/tobaccocontrol-2020-055933. Jafari D, Gandomi A, Makhnevich A, et al. (2021) Trajectories of Hypoxemia \& Respiratory System Mechanics of COVID19 ARDS in the NorthCARDS dataset. DOI: 10.1101/2021.01.26.21250492. 
Jakob CEM, Borgmann S, Duygu F, et al. (2020) First results of the 'Lean European Open Survey on SARS-CoV-2Infected Patients (LEOSS)'. Infection. DOI: 10.1007/s15010-020-01499-0.

Jehi L, Ji X, Milinovich A, et al. (2020) Development and validation of a model for individualized prediction of hospitalization risk in 4,536 patients with COVID-19. PLOS ONE Orueta JF (ed.) 15(8): e0237419. DOI:

10.1371/journal.pone.0237419.

Jin C, Gu J, Yuan Y, et al. (2020) Treatment of Six COVID-19 Patients with Convalescent Plasma. medRxiv: 2020.05.21.20109512. DOI: 10.1101/2020.05.21.20109512.

Jin X, Lian J-S, Hu J-H, et al. (2020) Epidemiological, clinical and virological characteristics of 74 cases of coronavirusinfected disease 2019 (COVID-19) with gastrointestinal symptoms. Gut 69(6): 1002-1009. DOI: 10.1136/gutjnl-2020$\underline{320926 .}$.

Joubert A, Andry F, Bertolotti A, et al. (2020) Distinguishing non severe cases of dengue from COVID-19 in the context of co-epidemics: A cohort study in a SARS-CoV-2 testing center on Reunion island. preprint, October. Infectious Diseases (except HIV/AIDS). DOI: 10.1101/2020.10.20.20214718.

Jun T, Nirenberg S, Kovatch P, et al. (2020) Sex-specificity of mortality risk factors among hospitalized COVID-19 patients in New York City: Prospective cohort study. medRxiv: 2020.07.29.20164640. DOI: 10.1101/2020.07.29.20164640.

Kalan ME, Ghobadi H, Taleb ZB, et al. (2020) Descriptive characteristics of hospitalized adult smokers and never-smokers with COVID-19. Tobacco Induced Diseases 18(May). DOI: 10.18332/tid/122759.

Kantele A, Laaveri T, Kareinen L, et al. (2020) SARS-CoV-2 infections among healthcare workers at Helsinki University Hospital, Finland, spring 2020: Serosurvey, symptoms and risk factors. Travel Med Infect Dis 39. DOI:

10.1016/j.tmaid.2020.101949.

Kara Polat A, Oguz Topal I, Karadag AS, et al. (2020) The impact of COVID-19 in patients with psoriasis: A multicenter study in Istanbul. Dermatol Ther NA. DOI: $10.1111 /$ dth.14691.

Kibler M, Carmona A, Marchandot B, et al. (2020) Risk and severity of COVID-19 and ABO blood group in transcatheter aortic valve patients. medRxiv: 2020.06.13.20130211. DOI: 10.1101/2020.06.13.20130211.

Killerby ME (2020) Characteristics Associated with Hospitalization Among Patients with COVID-19 — Metropolitan Atlanta, Georgia, March-April 2020. MMWR. Morbidity and Mortality Weekly Report69. DOI: 10.15585/mmwr.mm6925e1. Kim ES, Chin BS, Kang CK, et al. (2020) Clinical Course and Outcomes of Patients with Severe Acute Respiratory Syndrome Coronavirus 2 Infection: A Preliminary Report of the First 28 Patients from the Korean Cohort Study on COVID19. Journal of Korean Medical Science 35(13). DOI: 10.3346/jkms.2020.35.e142.

Kim H-J, Han D, Kim J-H, et al. (2020) An Easy-to-Use Machine Learning Model to Predict the Prognosis of Patients With COVID-19: Retrospective Cohort Study. Journal of Medical Internet Research 22(11): e24225. DOI: 10.2196/24225. Kim L, Garg S, O'Halloran A, et al. (2020) Interim Analysis of Risk Factors for Severe Outcomes among a Cohort of Hospitalized Adults Identified through the U.S. Coronavirus Disease 2019 (COVID-19)-Associated Hospitalization Surveillance Network (COVID-NET). medRxiv: 2020.05.18.20103390. DOI: 10.1101/2020.05.18.20103390. Kimmig LM, Wu D, Gold M, et al. (2020) IL-6 Inhibition in Critically III COVID-19 Patients Is Associated With Increased Secondary Infections. Frontiers in Medicine 7: 583897. DOI: 10.3389/fmed.2020.583897.

Kjetland EF, Kalleberg KT, Søraas CL, et al. (2020) Risk factors for community transmission of SARS-CoV-2. A cross- 
sectional study in 116,678 people. DOI: 10.1101/2020.12.23.20248514.

Klang E, Soffer S, Nadkarni G, et al. (2020) Sex Differences in Age and Comorbidities for COVID-19 Mortality in Urban New York City. SN Comprehensive Clinical Medicine 2(9): 1319-1322. DOI: 10.1007/s42399-020-00430-w.

Klang E, Kassim G, Soffer S, et al. (n.d.) Morbid Obesity as an Independent Risk Factor for COVID-19 Mortality in Hospitalized Patients Younger than 50. Obesity n/a(n/a). DOI: 10.1002/oby.22913.

Kolin DA, Kulm S and Elemento O (2020) Clinical and Genetic Characteristics of Covid-19 Patients from UK Biobank. medRxiv: 2020.05.05.20075507. DOI: 10.1101/2020.05.05.20075507.

Kortela E, Kirjavainen V, Ahava MJ, et al. (2020) Real-life clinical sensitivity of SARS-CoV-2 RT-PCR test in symptomatic patients. preprint, November. Infectious Diseases (except HIV/AIDS). DOI: 10.1101/2020.11.01.20223107.

Kronbichler A, Kresse D, Yoon S, et al. (2020) Asymptomatic patients as a source of COVID-19 infections: A systematic review and meta-analysis. International Journal of Infectious Diseases 98: 180-186. DOI: 10.1016/j.ijid.2020.06.052. Kuderer NM, Choueiri TK, Shah DP, et al. (2020) Clinical impact of COVID-19 on patients with cancer (CCC19): A cohort study. The Lancet 395(10241): 1907-1918. DOI: 10.1016/S0140-6736(20)31187-9.

Kumar A, Prasad G, Srivastav S, et al. (2020) A Retrospective Study on Efficacy and Safety of Guduchi Ghan Vati for Covid-19 Asymptomatic Patients. medRxiv: 2020.07.23.20160424. DOI: 10.1101/2020.07.23.20160424.

Kurashima K, Kagiyama N, Ishiguro T, et al. (2020) lgG antibody seroconversion and the clinical progression of COVID-19 pneumonia: A retrospective, cohort study. DOI: $10.1101 / 2020.07 .16 .20154088$.

Lamure S, Duléry R, Blasi RD, et al. (2020) Determinants of outcome in Covid-19 hospitalized patients with lymphoma: A retrospective multicentric cohort study. EClinicalMedicine 27. DOI: 10.1016/j.eclinm.2020.100549.

Lan F-Y, Suharlim C, Kales SN, et al. (2020) Association between SARS-CoV-2 infection, exposure risk and mental health among a cohort of essential retail workers in the United States. medRxiv: 2020.06.08.20125120. DOI:

10.1101/2020.06.08.20125120.

Lassale C, Gaye B, Hamer M, et al. (2020) Ethnic disparities in hospitalisation for COVID-19 in England: The role of socioeconomic factors, mental health, and inflammatory and pro-inflammatory factors in a community-based cohort study. Brain, Behavior, and Immunity 88: 44-49. DOI: 10.1016/j.bbi.2020.05.074.

Leister I, Ponocny-Seliger E, Kollaritsch H, et al. (2021) Antibody seroprevalence and rate of asymptomatic infections with SARS-CoV-2 in Austrian hospital personnel. DOI: 10.1101/2021.02.01.21250898.

Lenka J, Chhabria MS, Sharma N, et al. (2020) Clinical characteristics and outcomes of critically ill patients with COVID-19 in a tertiary community hospital in upstate New York. medRxiv: 2020.06.18.20135046. DOI:

10.1101/2020.06.18.20135046.

Lewnard JA, Mora AM, Nkwocha O, et al. (2021) Prevalence and clinical profile of SARS-CoV-2 infection among farmworkers in Monterey County, California: June-November, 2020. DOI: 10.1101/2020.12.27.20248894.

Lévy Y, Wiedemann A, Hejblum BP, et al. (2020) CD177, a specific marker of neutrophil activation, is a hallmark of COVID-19 severity and death. preprint, December. Allergy; Immunology. DOI: 10.1101/2020.12.12.20246934.

Li F, Cai Y, Gao C, et al. (2020) Clinical Course And Risk Factors For In-hospital Death In Critical COVID-19 In Wuhan, China. preprint, September. Public; Global Health. DOI: 10.1101/2020.09.26.20189522.

Li J, Chen Y, Chen S, et al. (2020) Derivation and validation of a prognostic model for predicting in-hospital mortality in 
patients admitted with COVID-19 in Wuhan, China: The PLANS (Platelet Lymphocyte Age Neutrophil Sex) model. medRxiv: 2020.05.13.20100370. DOI: 10.1101/2020.05.13.20100370.

Li J, Li S, Cai Y, et al. (2020) Epidemiological and Clinical Characteristics of 17 Hospitalized Patients with 2019 Novel Coronavirus Infections Outside Wuhan, China. medRxiv: 2020.02.11.20022053. DOI: 10.1101/2020.02.11.20022053. Li J, Long X, Zhang Q, et al. (2020) Tobacco smoking confers risk for severe COVID-19 unexplainable by pulmonary imaging. Journal of Internal Medicine: joim.13190. DOI: 10.1111/joim.13190.

Li J, Long X, Zhu C, et al. (n.d.) Olfactory Dysfunction in Recovered Coronavirus Disease 2019 (COVID-19) Patients. Movement Disorders n/a(n/a). DOI: 10.1002/mds.28172.

Lian J, Jin X, Hao S, et al. (n.d.) Analysis of Epidemiological and Clinical Features in Older Patients With Coronavirus Disease 2019 (COVID-19) Outside Wuhan. Clinical Infectious Diseases. DOI: 10.1093/cid/ciaa242.

Liao Y, Feng Y, Wang B, et al. (2020) Clinical Characteristics and Risk factors for developed COVID-19 patients transferring to designated hospital from Jianghan Fangcang shelter Hospital: A retrospective, observational study. medRxiv: 2020.04.21.20074724. DOI: 10.1101/2020.04.21.20074724.

Lin KJ, Schneeweiss S, Tesfaye H, et al. (2020) Pharmacotherapy for Hospitalized Patients with COVID-19: Treatment Patterns by Disease Severity. Drugs 80: 1961-1972. DOI: 10.1007/s40265-020-01424-7.

Liu R, Ming X, Xu O, et al. (2020) Association of Cardiovascular Manifestations with In-hospital Outcomes in Patients with COVID-19: A Hospital Staff Data. medRxiv: 2020.02.29.20029348. DOI: 10.1101/2020.02.29.20029348.

Liu W, Tao Z-W, Wang L, et al. (2020) Analysis of factors associated with disease outcomes in hospitalized patients with 2019 novel coronavirus disease. Chinese Medical Journal 133(9): 1032-1038. DOI: 10.1097/CM9.0000000000000775. Lohia P, Sreeram K, Nguyen P, et al. (2021) Preexisting respiratory diseases and clinical outcomes in COVID-19: A multihospital cohort study on predominantly African American population. Respir Res 22. DOI: 10.1186/s12931-02101647-6.

Lopez-Medrano F, Perez-Jacoiste Asin MA, Fernandez-Ruiz M, et al. (2020) Combination therapy with tocilizumab and corticosteroids for aged patients with severe COVID-19 pneumonia: A single-center retrospective study. preprint, September. Infectious Diseases (except HIV/AIDS). DOI: 10.1101/2020.09.26.20202283.

Louis S, Dhawan A, Newey C, et al. (2020) Continuous Electroencephalography (cEEG) Characteristics and Acute Symptomatic Seizures in COVID-19 Patients. medRxiv: 2020.05.26.20114033. DOI: 10.1101/2020.05.26.20114033. Lowe KE, Zein J, Hatipoglu U, et al. (2021) Association of Smoking and Cumulative Pack-Year Exposure With COVID-19 Outcomes in the Cleveland Clinic COVID-19 Registry. JAMA Intern Med NA. DOI: 10.1001/jamainternmed.2020.8360. Lubetzky M, Aull M, Craig-Shapiro R, et al. (2020) Kidney Allograft Recipients Diagnosed with Coronavirus Disease-2019: A Single Center Report. medRxiv: 2020.04.30.20086462. DOI: 10.1101/2020.04.30.20086462.

Luo H, Liu S, Wang Y, et al. (2020) Age differences in clinical features and outcomes in patients with COVID-19, Jiangsu, China: A retrospective, multi-center cohort study. medRxiv: 2020.06.01.20086025. DOI: 10.1101/2020.06.01.20086025. Luo J, Rizvi H, Preeshagul IR, et al. (2020) COVID-19 in patients with lung cancer. Annals of Oncology 31(10): 13861396. DOI: 10.1016/j.annonc.2020.06.007.

Lusignan S de, Dorward J, Correa A, et al. (2020) Risk factors for SARS-CoV-2 among patients in the Oxford Royal College of General Practitioners Research and Surveillance Centre primary care network: A cross-sectional study. The 
Lancet Infectious Diseases: S1473309920303716. DOI: 10.1016/S1473-3099(20)30371-6.

Madariaga MLL, Guthmiller J, Schrantz S, et al. (2020) Clinical predictors of donor antibody titer and correlation with recipient antibody response in a COVID-19 convalescent plasma clinical trial. medRxiv: 2020.06.21.20132944. DOI: 10.1101/2020.06.21.20132944.

Magagnoli J, Narendran S, Pereira F, et al. (2020) Outcomes of Hydroxychloroquine Usage in United States Veterans Hospitalized with COVID-19. Med: S2666634020300064. DOI: 10.1016/j.medj.2020.06.001.

Magleby R, Westblade LF, Trzebucki A, et al. (n.d.) Impact of SARS-CoV-2 Viral Load on Risk of Intubation and Mortality Among Hospitalized Patients with Coronavirus Disease 2019. Clinical Infectious Diseases. DOI: 10.1093/cid/ciaa851. Makaronidis J, Mok J, Balogun N, et al. (2020) Seroprevalence of SARS-CoV-2 antibodies in people with an acute loss in their sense of smell and/or taste in a community-based population in London, UK: An observational cohort study. PLOS Medicine 17(10): e1003358. DOI: 10.1371/journal.pmed.1003358.

Mamtani M, Athavale AM, Abraham M, et al. (2020) ASSOCIATION OF HYPERGLYCEMIA WITH HOSPITAL MORTALITY IN COVID-19 PATIENTS WITHOUT DIABETES: A COHORT STUDY. medRxiv: 2020.08.31.20185157. DOI: $10.1101 / 2020.08 .31 .20185157$.

Mancilla-Galindo J, Vera-Zertuche JM, Navarro-Cruz AR, et al. (2020) Development and Validation of the Patient History COVID-19 (PH-Covid19) Scoring System: A Multivariable Prediction Model of Death in Mexican Patients with COVID-19. medRxiv: 2020.09.05.20189142. DOI: 10.1101/2020.09.05.20189142.

Mangera Z, Lewis A, Hutchinson J, et al. (2017) Smoking prevalence in UK hospital admissions from a national observational study. European Respiratory Journal 50(suppl 61). DOI: 10.1183/1393003.congress-2017.PA1268. Mansour A, Sajjadi-Jazi SM, Kasaeian A, et al. (2020) Clinical characteristics and outcomes of diabetics hospitalized for COVID-19 infection: A single-centered, retrospective, observational study. EXCLI j 19: 1533-1543. DOI: 10.17179/excli2020-2988.

Maraschini A, Corsi E, Salvatore MA, et al. (2020) Coronavirus and birth in Italy: Results of a national population-based cohort study. medRxiv: 2020.06.11.20128652. DOI: 10.1101/2020.06.11.20128652.

Marcos M, Belhassen-Garcia M, Puente AS, et al. (2020) Development of a severity of disease score and classification model by machine learning for hospitalized COVID-19 patients. medRxiv: 2020.07.13.20150177. DOI:

10.1101/2020.07.13.20150177.

Martinez-Lacalzada M, Viteri-Noël LA, Manzano L, et al. (2020) Predicting critical illness on initial diagnosis of COVID-19: Development and validation of the PRIORITY model for outpatient applicability. DOI: $10.1101 / 2020.11 .27 .20237966$. Martinez-Portilla RJ, Sotiriadis A, Torres-Torres J, et al. (2020) Risk factors for mortality in pregnant women with SARSCoV-2 infection. medRxiv: 2020.05.31.20107276. DOI: 10.1101/2020.05.31.20107276.

Martinez-Resendez MF, Castilleja-Leal F, Torres-Quintanilla A, et al. (2020) Initial experience in Mexico with convalescent plasma in COVID-19 patients with severe respiratory failure, a retrospective case series. medRxiv: 2020.07.14.20144469. DOI: $10.1101 / 2020.07 .14 .20144469$.

Martini F, D’Alessio A, Bracchi F, et al. (2020) On Cancer, COVID-19, and CT Scans: A Monocentric Retrospective Study. Journal of Clinical Medicine 9(12): 3935. DOI: 10.3390/jcm9123935.

Martin-Jimenez P, Munoz-Garcia MI, Seoane D, et al. (2020) Cognitive impairment is a common comorbidity in COVID-19 
deceased patients. A hospital-based retrospective cohort study. medRxiv: 2020.06.08.20125872. DOI:

10.1101/2020.06.08.20125872.

Maucourant C, Filipovic I, Ponzetta A, et al. (2020) Natural killer cell activation related to clinical outcome of COVID-19. medRxiv: 2020.07.07.20148478. DOI: 10.1101/2020.07.07.20148478.

Márquez-Salinas A, Fermín-Martínez CA, Antonio-Villa NE, et al. (2020) Adaptive metabolic and inflammatory responses identified using accelerated aging metrics are linked to adverse outcomes in severe SARS-CoV-2 infection. preprint, November. Geriatric Medicine. DOI: 10.1101/2020.11.03.20225375.

McGrail DE and Edwards D (2020) COVID-19 Case Series at UnityPoint Health St. Lukes Hospital in Cedar Rapids, IA. medRxiv: 2020.07.17.20156521. DOI: 10.1101/2020.07.17.20156521.

McQueenie R, Foster H, Jani BD, et al. (2020) Multimorbidity, Polypharmacy, and COVID-19 infection within the UK Biobank cohort. medRxiv: 2020.06.10.20127563. DOI: 10.1101/2020.06.10.20127563.

Meini S, Fortini A, Andreini R, et al. (n.d.) The Paradox of the Low Prevalence of Current Smokers Among Covid-19 Patients Hospitalized in Non-Intensive Care Wards: Results From an Italian Multicenter Case-Control Study. Nicotine \& Tobacco Research. DOI: 10.1093/ntr/ntaa188.

Mejia-Vilet JM, Cordova-Sanchez BM, Fernandez-Camargo D, et al. (2020) A Risk Score to Predict Admission to Intensive Care Unit in Patients With COVID-19: The ABC-GOALS Score. medRxiv: 2020.05.12.20099416. DOI:

10.1101/2020.05.12.20099416.

Melo AC de, Thuler LCS, Silva JL da, et al. (2020) Cancer inpatient with COVID-19: A report from the Brazilian National Cancer Institute. medRxiv: 2020.06.27.20141499. DOI: 10.1101/2020.06.27.20141499.

Mendes A, Herrmann FR, Genton L, et al. (2021) Incidence, characteristics and clinical relevance of acute stroke in old patients hospitalized with COVID-19. BMC geriatr21. DOI: 10.1186/s12877-021-02006-2.

Mendy A, Apewokin S, Wells AA, et al. (2020) Factors Associated with Hospitalization and Disease Severity in a Racially and Ethnically Diverse Population of COVID-19 Patients. medRxiv: 2020.06.25.20137323. DOI:

10.1101/2020.06.25.20137323.

Merkely B, Szabó AJ, Kosztin A, et al. (2020) Novel coronavirus epidemic in the Hungarian population, a cross-sectional nationwide survey to support the exit policy in Hungary. GeroScience. DOI: 10.1007/s11357-020-00226-9.

Merzon E, Tworowski D, Gorohovski A, et al. (2020) Low plasma 25(OH) vitamin D3 level is associated with increased risk of COVID-19 infection: An Israeli population-based study. medRxiv: 2020.07.01.20144329. DOI:

10.1101/2020.07.01.20144329.

Miyara M, Tubach F, Martinez V, et al. (2020) Low rate of daily smokers in patients with symptomatic COVID-19. medRxiv: 2020.06.10.20127514. DOI: 10.1101/2020.06.10.20127514.

Mo P, Xing Y, Xiao Y, et al. (n.d.) Clinical characteristics of refractory COVID-19 pneumonia in Wuhan, China. Clinical Infectious Diseases. DOI: 10.1093/cid/ciaa270.

Modrák M, Bürkner P-C, Sieger T, et al. (2020) Detailed disease progression of 213 patients hospitalized with Covid-19 in the Czech Republic: An exploratory analysis. DOI: 10.1101/2020.12.03.20239863.

Mohamed-Hussein A, Galal I, Saad M, et al. (2020) Post-COVID-19 Functional Status: Relation to age, smoking, hospitalization and comorbidities. medRxiv: 2020.08.26.20182618. DOI: $10.1101 / 2020.08 .26 .20182618$. 
Mohamud AY, Griffith B, Rehman M, et al. (2020) Intraluminal Carotid Artery Thrombus in COVID-19: Another Danger of Cytokine Storm? American Journal of Neuroradiology. DOI: 10.3174/ajnr.A6674.

Molenaar NM, Rommel A-S, Witte L de, et al. (2021) Seroprevalence of SARS-CoV-2 during pregnancy and associated outcomes: Results from an ongoing prospective cohort study, New York City. DOI: 10.1101/2021.02.01.21250943. Monteiro ACC, Suri R, Emeruwa IO, et al. (2020) Obesity and Smoking as Risk Factors for Invasive Mechanical Ventilation in COVID-19: A Retrospective, Observational Cohort Study. medRxiv: 2020.08.12.20173849. DOI:

10.1101/2020.08.12.20173849.

Mora AM, Lewnard JA, Kogut K, et al. (2021) Risk factors for SARS-CoV-2 infection among farmworkers in Monterey County, California. DOI: 10.1101/2021.02.01.21250963.

Morshed MS, Mosabbir AA, Chowdhury P, et al. (2020) Clinical manifestations of patients with Coronavirus Disease 2019 (COVID-19) attending at hospitals in Bangladesh. medRxiv: 2020.07.30.20165100. DOI: 10.1101/2020.07.30.20165100. Motta JK, Ogunnaike RO, Shah R, et al. (2020) Clinical Outcomes With the Use of Prophylactic Versus Therapeutic Anticoagulation in COVID-19. medRxiv: 2020.07.20.20147769. DOI: 10.1101/2020.07.20.20147769.

Muñoz FJT, García-Guijarro E, García-Domingo P, et al. (2020) A safe protocol to identify low risk patients with COVID-19 pneumonia for outpatient management. medRxiv: 2020.12.15.20229286. DOI: $\underline{10.1101 / 2020.12 .15 .20229286}$.

Murray E (2020) Causation in Smoking and COVID-19. Available at: https://twitter.com/EpiEllie/status/1258607277357006849?s=20.

Mutambudzi M, Niedzwiedz CL, Macdonald EB, et al. (2020) Occupation and risk of severe COVID-19: Prospective cohort study of 120,075 UK Biobank participants. medRxiv: 2020.05.22.20109892. DOI: 10.1101/2020.05.22.20109892. Nezhadmoghadam F and Tamez-Peña J (2021) Unsupervised Discovery of Risk Profiles on Negative and Positive COVID-19 Hospitalized Patients. DOI: 10.1101/2020.12.30.20248908.

Nguyen AB, Upadhyay GA, Chung B, et al. (2020) Outcomes and Cardiovascular Comorbidities in a Predominantly African-American Population with COVID-19. medRxiv: 2020.06.28.20141929. DOI: $\underline{10.1101 / 2020.06 .28 .20141929}$. Nicholson CJ, Wooster L, Sigurslid HH, et al. (2020) Estimating Risk of Mechanical Ventilation and Mortality Among Adult COVID-19 patients Admitted to Mass General Brigham: The VICE and DICE Scores. medRxiv: 2020.09.14.20194670. DOI: $\underline{10.1101 / 2020.09 .14 .20194670 .}$.

Niedzwiedz CL, O'Donnell CA, Jani BD, et al. (2020) Ethnic and socioeconomic differences in SARS-CoV-2 infection: Prospective cohort study using UK Biobank. BMC Medicine 18(1): 160. DOI: 10.1186/s12916-020-01640-8. Nuño M, García Y, Rajasekar G, et al. (2021) COVID-19 Hospitalizations in Five California Hospitals. DOI: 10.1101/2021.01.29.21250788.

Núñez-Gil IJJ, Fernández-Ortiz A, Eid CM, et al. (2020) Underlying heart diseases and acute COVID-19 outcomes. Cardiology Journal 0(0). DOI: 10.5603/cj.a2020.0183.

Oakes JM, Fuchs RM, Gardner JD, et al. (2018) Nicotine and the renin-angiotensin system. Am. J. Physiol. - Regul. Integr. Comp. Physiol 315: 895-906. DOI: 10.1152/ajpregu.00099.2018.

Odani S (2018) Tobacco Product Use Among Military Veterans — United States, 2010-2015. MMWR. Morbidity and Mortality Weekly Report 67. DOI: 10.15585/mmwr.mm6701a2.

O'Gallagher K, Shek A, Bean DM, et al. (2020) Pre-existing cardiovascular disease rather than cardiovascular risk factors 
drives mortality in COVID-19. preprint, December. Cardiovascular Medicine. DOI: 10.1101/2020.12.02.20242933.

Olivares F, Munoz D, Fica A, et al. (2020) Covid-19 in Chile. The experience of a Regional reference Center. Preliminary report. medRxiv: 2020.06.14.20130898. DOI: $\underline{10.1101 / 2020.06 .14 .20130898 .}$

Oliveira E, Parikh A, Lopez-Ruiz A, et al. (2020) ICU Outcomes and Survival in Patients with Severe COVID-19 in the Largest Health Care System in Central Florida. medRxiv: 2020.08.25.20181909. DOI: 10.1101/2020.08.25.20181909. Omrani AS, Almaslamani MA, Daghfal J, et al. (2020) The First Consecutive 5000 Patients with Coronavirus Disease 2019 from Qatar; a Nation-wide Cohort Study. medRxiv: 2020.07.15.20154690. DOI: 10.1101/2020.07.15.20154690.

O’Reilly GM, Mitchell RD, Mitra B, et al. (2020) Epidemiology and clinical features of emergency department patients with suspected and confirmed <span style="font-variant:Small-caps;">COVID</span>-19: A multisite report from the <span style="font-variant:Small-caps;">COVID-19 Emergency Department</span> Quality Improvement Project for July 2020 ( <span style="font-variant:Small-caps;">COVED</span> -3). Emergency Medicine Australasia: 1742-6723.13651. DOI: 10.1111/1742-6723.13651.

Organisation WH (n.d.) Laboratory testing for 2019 novel coronavirus (2019-nCoV) in suspected human cases. Available at: https://www.who.int/publications-detail-redirect/10665-331501.

Ouyang J, Shan X, Wang X, et al. (2020) Clinical characteristics of COVID-19 and the model for predicting the occurrence of critically ill patients: A retrospective cohort study. medRxiv: 2020.08.13.20173799. DOI: 10.1101/2020.08.13.20173799. Palaiodimos L, Kokkinidis DG, Li W, et al. (2020) Severe obesity, increasing age and male sex are independently associated with worse in-hospital outcomes, and higher in-hospital mortality, in a cohort of patients with COVID-19 in the Bronx, New York. Metabolism 108: 154262. DOI: 10.1016/j.metabol.2020.154262.

Paleiron N, Mayet A, Marbac V, et al. (2021) Impact of Tobacco Smoking on the risk of COVID-19.A large scale retrospective cohort study. Nicotine Tob Res NA. DOI: 10.1093/ntr/ntab004.

Pan A, Khan O, Meeks J, et al. (2020) Disparities in COVID-19 Hospitalizations and Mortality among Black and Hispanic Patients: Cross-Sectional Analysis from the Greater Houston Metropolitan Area. medRxiv: 2020.08.19.20177956. DOI: 10.1101/2020.08.19.20177956.

Pandolfi L, Fossali T, Frangipane V, et al. (2020) Broncho-alveolar inflammation in COVID-19 patients: A correlation with clinical outcome. medRxiv: 2020.07.17.20155978. DOI: 10.1101/2020.07.17.20155978.

Park BE, Lee JH, Park HK, et al. (2021) Impact of Cardiovascular Risk Factors and Cardiovascular Diseases on Outcomes in Patients Hospitalized with COVID-19 in Daegu Metropolitan City. J Korean Med Sci 36. DOI: 10.3346/jkms.2021.36.e15. Parra-Bracamonte GM, Lopez-Villalobos N and Parra-Bracamonte FE (2020) Clinical characteristics and risk factors for mortality of patients with COVID-19 in a large data set from Mexico. Annals of Epidemiology 52: 93-98.e2. DOI:

10.1016/j.annepidem.2020.08.005.

Parrotta E, Kister I, Charvet L, et al. (2020) COVID-19 outcomes in MS: Observational study of early experience from NYU Multiple Sclerosis Comprehensive Care Center. Neurology - Neuroimmunology Neuroinflammation 7(5): e835. DOI: 10.1212/NXI.0000000000000835.

Patanavanich R and Glantz SA (2020) Smoking is Associated with COVID-19 Progression: A Meta-Analysis. medRxiv. DOI: 10.14171/j.2095-5944.sg.2014.02.004.

Patel M, Chowdhury J, Mills N, et al. (2020) ROX Index Predicts Intubation in Patients with COVID-19 Pneumonia and 
Moderate to Severe Hypoxemic Respiratory Failure Receiving High Flow Nasal Therapy. medRxiv: 2020.06.30.20143867. DOI: $10.1101 / 2020.06 .30 .20143867$.

Perico L, Tomasoni S, Peracchi T, et al. (2020) COVID-19 and lombardy: TESTing the impact of the first wave of the pandemic. EBioMedicine 61. DOI: 10.1016/j.ebiom.2020.103069.

Perrone F, Piccirillo MC, Ascierto PA, et al. (2020) Tocilizumab for patients with COVID-19 pneumonia. The TOCIVID-19 prospective phase 2 trial. medRxiv: 2020.06.01.20119149. DOI: 10.1101/2020.06.01.20119149.

Peters EJ, Collard D, Assen S van, et al. (2020) Outcomes of Persons With COVID-19 in Hospitals With and Without Standard Treatment With (Hydroxy)chloroquine. medRxiv: 2020.08.14.20173369. DOI: 10.1101/2020.08.14.20173369. Petrilli CM, Jones SA, Yang J, et al. (2020) Factors associated with hospital admission and critical illness among 5279 people with coronavirus disease 2019 in New York City: Prospective cohort study. BMJ 369. DOI: 10.1136/bmj.m1966. Philipose Z, Smati N, Wong CSJ, et al. (2020) Obesity, old age and frailty are the true risk factors for COVID-19 mortality and not chronic disease or ethnicity in Croydon. medRxiv: 2020.08.12.20156257. DOI: 10.1101/2020.08.12.20156257. Polubriaginof F, Salmasian H, Albert DA, et al. (2018) Challenges with Collecting Smoking Status in Electronic Health Records. AMIA Annual Symposium Proceedings 2017: 1392-1400. Available at: https://www.ncbi.nlm.nih.gov/pmc/articles/PMC5977725/ (accessed 25 September 2020). Pongpirul WA, Wiboonchutikul S, Charoenpong L, et al. (2020) Clinical course and potential predicting factors of pneumonia of adult patients with coronavirus disease 2019 (COVID-19): A retrospective observational analysis of 193 confirmed cases in Thailand. medRxiv: 2020.06.24.20139642. DOI: $10.1101 / 2020.06 .24 .20139642$.

Prats-Uribe A, Tobed M, Villacampa JM, et al. (2021) Timing of elective tracheotomy and duration of mechanical ventilation amongst patients admitted to intensive care with severe COVID-19: A multicentre prospective cohort study. DOI: 10.1101/2021.01.22.21249651.

Qi D, Yan X, Tang X, et al. (2020) Epidemiological and clinical features of 2019-nCoV acute respiratory disease cases in Chongqing municipality, China: A retrospective, descriptive, multiple-center study. medRxiv: 2020.03.01.20029397. DOI: 10.1101/2020.03.01.20029397.

Qu J, Chang LK, Tang X, et al. (2020) Clinical characteristics of COVID-19 and its comparison with influenza pneumonia. Acta Clinica Belgica 0(0): 1-9. DOI: 10.1080/17843286.2020.1798668.

Quan D, Luna Wong L, Shallal A, et al. (2021) Impact of Race and Socioeconomic Status on Outcomes in Patients Hospitalized with COVID-19. J Gen Intern Med NA. DOI: 10.1007/s11606-020-06527-1.

Raines AM, Tock JL, McGrew SJ, et al. (2021) Correlates of death among SARS-CoV-2 positive veterans: The contribution of lifetime tobacco use. Addictive Behaviors 113: 106692. DOI: 10.1016/j.addbeh.2020.106692.

Raisi-Estabragh Z, McCracken C, Bethell MS, et al. (n.d.) Greater risk of severe COVID-19 in Black, Asian and Minority Ethnic populations is not explained by cardiometabolic, socioeconomic or behavioural factors, or by $25(\mathrm{OH})$-vitamin $\mathrm{D}$ status: Study of 1326 cases from the UK Biobank. Journal of Public Health. DOI: 10.1093/pubmed/fdaa095.

Rajter JC, Sherman M, Fatteh N, et al. (2020) ICON (Ivermectin in COvid Nineteen) study: Use of Ivermectin is Associated with Lower Mortality in Hospitalized Patients with COVID19. medRxiv: 2020.06.06.20124461. DOI:

10.1101/2020.06.06.20124461.

Ramachandran P, Kathirvelu B, Chakraborti A, et al. (2020) COVID-19 in Cancer Patients From New York City: A 
Comparative Single Center Retrospective Analysis. Cancer Control 27(1): 107327482096045. DOI:

\subsection{7/1073274820960457.}

Ramlall V, Thangaraj P, Meydan C, et al. (2020) Identification of Immune complement function as a determinant of adverse SARS-CoV-2 infection outcome. medRxiv: 2020.05.05.20092452. DOI: 10.1101/2020.05.05.20092452.

Rashid M, Wu J, Timmis A, et al. (2020) Clinical Characteristics and Outcomes of COVID-19 Positive Acute Coronary Syndrome Patients; a multisource Electronic Healthcare Records Study from England. medRxiv: 2020.08.20.20175091. DOI: $10.1101 / 2020.08 .20 .20175091$.

REACT Study Investigators, Riley S, Ainslie KEC, et al. (2020) Community prevalence of SARS-CoV-2 virus in England during May 2020: REACT study. DOI: 10.1101/2020.07.10.20150524.

Regina J, Papadimitriou-Olivgeris M, Burger R, et al. (2020) Epidemiology, risk factors and clinical course of SARS-CoV-2 infected patients in a Swiss university hospital: An observational retrospective study. medRxiv: 2020.05.11.20097741.

DOI: $10.1101 / 2020.05 .11 .20097741$.

Reiter T, Pajenda S, Wagner L, et al. (2020) Covid-19 serology in nephrology health care workers. medRxiv: 2020.07.21.20136218. DOI: 10.1101/2020.07.21.20136218.

Ren HG, Guo X, Tu L, et al. (2020) Clinical Characteristics and Risk Factors for Myocardial Injury and Arrhythmia in COVID-19 patients. preprint, December. Cardiovascular Medicine. DOI: 10.1101/2020.11.30.20190926.

Ren HG, Guo X, Blighe K, et al. (2020) Risk Factors for ICU Admission, Mechanical Ventilation and Mortality in Hospitalized Patients with COVID-19 in Hubei, China. medRxiv: 2020.08.31.20184952. DOI:

$10.1101 / 2020.08 .31 .20184952$.

Rentsch CT, Kidwai-Khan F, Tate JP, et al. (2020) Covid-19 Testing, Hospital Admission, and Intensive Care Among 2,026,227 United States Veterans Aged 54-75 Years. medRxiv: 2020.04.09.20059964. DOI: 10.1101/2020.04.09.20059964.

Rentsch CT, Beckman JA, Tomlinson L, et al. (2020) Early initiation of prophylactic anticoagulation for prevention of COVID-19 mortality: A nationwide cohort study of hospitalized patients in the United States. preprint, December. Infectious Diseases (except HIV/AIDS). DOI: 10.1101/2020.12.09.20246579.

Rica R de la, Borges M, Aranda M, et al. (2020) Low albumin levels are associated with poorer outcomes in a case series of COVID-19 patients in Spain: A retrospective cohort study. medRxiv: 2020.05.07.20094987. DOI:

\subsection{1/2020.05.07.20094987.}

Richard A, Wisniak A, Perez-Saez J, et al. (2020) Seroprevalence of anti-SARS-CoV-2 lgG antibodies, risk factors for infection and associated symptoms in Geneva, Switzerland: A population-based study. medRxiv: 2020.12.16.20248180. DOI: $10.1101 / 2020.12 .16 .20248180$.

Richardson S, Hirsch JS, Narasimhan M, et al. (2020) Presenting Characteristics, Comorbidities, and Outcomes Among 5700 Patients Hospitalized With COVID-19 in the New York City Area. JAMA 323(20): 2052-2059. DOI:

\subsection{1/jama.2020.6775.}

Rimland CA, Morgan CE, Bell GJ, et al. (2020) Clinical characteristics and early outcomes in patients with COVID-19 treated with tocilizumab at a United States academic center. medRxiv: 2020.05.13.20100404. DOI:

10.1101/2020.05.13.20100404. 
Rizzo S, Chawla D, Zalocusky K, et al. (2020) Descriptive epidemiology of 16,780 hospitalized COVID-19 patients in the United States. medRxiv: 2020.07.17.20156265. DOI: 10.1101/2020.07.17.20156265.

Robilotti EV, Babady NE, Mead PA, et al. (2020) Determinants of Severity in Cancer Patients with COVID-19 Illness. medRxiv: 2020.05.04.20086322. DOI: 10.1101/2020.05.04.20086322.

Robinson L, Wang L, Fu X, et al. (2021) COVID-19 severity in asthma patients: A multi-center matched cohort study. Journal of Allergy and Clinical Immunology 147(2): AB241. DOI: 10.1016/.j.jaci.2020.12.026.

Roederer T, Mollo B, Vincent C, et al. (2021) Seroprevalence and risk factors of exposure to COVID-19 in homeless people in Paris, France: A cross-sectional study. The Lancet Public Health: S2468266721000013. DOI: 10.1016/S24682667(21)00001-3.

Romão VC, Oliveira-Ramos F, Cruz-Machado AR, et al. (2020) A COVID-19 outbreak in a rheumatology department upon the early days of the pandemic. medRxiv: 2020.06.05.20107011. DOI: $10.1101 / 2020.06 .05 .20107011$.

Rossi B, Nguyen LS, Zimmermann P, et al. (2020) Effect of tocilizumab in hospitalized patients with severe pneumonia COVID-19: A cohort study. medRxiv: 2020.06.06.20122341. DOI: 10.1101/2020.06.06.20122341.

Rowlands AV, Gillies C, Chudasama Y, et al. (2020) Association of working shifts, inside and outside of healthcare, with risk of severe COVID-19: An observational study. DOI: 10.1101/2020.12.16.20248243.

Rubio-Rivas M, Ronda M, Padulles A, et al. (2020) Beneficial Effect of Corticosteroids in Preventing Mortality in Patients Receiving Tocilizumab to Treat Severe COVID-19 IIIness. medRxiv: 2020.08.31.20182428. DOI:

\subsection{1/2020.08.31.20182428.}

Russell B, Moss C, Papa S, et al. (2020) Factors Affecting COVID-19 Outcomes in Cancer Patients: A First Report From Guy's Cancer Center in London. Frontiers in Oncology 10: 1279. DOI: 10.3389/fonc.2020.01279.

Saeed GA, Al Helali AA, Almazrouei S, et al. (2020) Chest CT features of COVID-19 in the region of Abu Dhabi, UAE-A single institute study. preprint, November. Radiology; Imaging. DOI: 10.1101/2020.11.14.20229096.

Salama C, Han J, Yau L, et al. (2021) Tocilizumab in Patients Hospitalized with Covid-19 Pneumonia. New England Journal of Medicine 384(1): 20-30. DOI: 10.1056/NEJMoa2030340.

Salerno S, Zhao Z, Sankar SP, et al. (2020) Understanding the patterns of repeated testing for COVID-19: Association with patient characteristics and outcomes. medRxiv: 2020.07.26.20162453. DOI: 10.1101/2020.07.26.20162453.

Salton F, Confalonieri P, Santus P, et al. (2020) Prolonged low-dose methylprednisolone in patients with severe COVID19 pneumonia. medRxiv: 2020.06.17.20134031. DOI: 10.1101/2020.06.17.20134031.

Sami R, Soltaninejad F, Amra B, et al. (2020) A one-year hospital-based prospective COVID-19 open-cohort in the Eastern Mediterranean region: The Khorshid COVID Cohort (KCC) study. medRxiv: 2020.05.11.20096727. DOI:

10.1101/2020.05.11.20096727.

Santos C, Rhee Y, Hollinger E, et al. (2020) Comparative Incidence and Outcomes of COVID-19 in Kidney or KidneyPancreas Transplant Recipients Versus Kidney or Kidney-Pancreas Waitlisted Patients: A Pilot Study. medRxiv: 2020.07.20.20157990. DOI: 10.1101/2020.07.20.20157990.

Saurabh S, Verma MK, Gautam V, et al. (2021) Tobacco, alcohol use and other risk factors for developing symptomatic COVID-19 vs asymptomatic SARS-CoV-2 infection: A case-control study from western Rajasthan, India. Trans $R$ Soc Trop Med Hyg NA. DOI: $10.1093 /$ trstmh/traa172. 
Savarraj JP, Burkett AB, Hinds SN, et al. (n.d.) Three-month outcomes in hospitalized COVID-19 patients.: 7. DOI: 10.1101/2020.10.16.20211029.

Schneeweiss MC, Leonard S, Weckstein A, et al. (2020) Renin-Angiotensin-Aldosterone-System inhibitor use in patients with COVID-19 infection and prevention of serious events: A cohort study in commercially insured patients in the US. medRxiv: 2020.07.22.20159855. DOI: 10.1101/2020.07.22.20159855.

Schoeman D and Fielding BC (2019) Coronavirus envelope protein: Current knowledge. Virology Journal 16(1): 69. DOI: 10.1186/s12985-019-1182-0.

Schubl SD, Figueroa C, Palma AM, et al. (2020) Risk Factors for SARS-CoV-2 Seropositivity in a Health Care Worker Population. DOI: $10.1101 / 2020.12 .17 .20248430$.

Senkal N (2020) Chronic ACE Inhibitor use is Associated with Decreased Odds of Severe Disease in Patients with COVID-19. The Anatolian Journal of Cardiology. DOI: 10.14744/AnatolJCardiol.2020.57431.

Serling-Boyd N, D'Silva KM, Hsu TY, et al. (2020) Coronavirus disease 2019 outcomes among patients with rheumatic diseases 6 months into the pandemic. Annals of the Rheumatic Diseases: annrheumdis-2020-219279. DOI:

\subsection{6/annrheumdis-2020-219279.}

Shade JK, Doshi AN, Sung E, et al. (2021) COVID-HEART: Development and Validation of a Multi-Variable Model for Real-Time Prediction of Cardiovascular Complications in Hospitalized Patients with COVID-19. DOI:

\subsection{1/2021.01.03.21249182.}

Shah SJ, Barish PN, Prasad PA, et al. (2020) Clinical features, diagnostics, and outcomes of patients presenting with acute respiratory illness: A comparison of patients with and without COVID-19. medRxiv: 2020.05.02.20082461. DOI: 10.1101/2020.05.02.20082461.

Shahab L, Brose LS and West R (2013) Novel delivery systems for nicotine replacement therapy as an aid to smoking cessation and for harm reduction: Rationale, and evidence for advantages over existing systems. CNS Drugs 27(12): 1007-1019. DOI: 10.1007/s40263-013-0116-4.

Sharma AK, Ahmed A, Baig VN, et al. (2020) Characteristics and Outcomes of Hospitalized Young Adults with Mild to Moderate Covid-19 at a University Hospital in India. medRxiv: 2020.06.02.20106310. DOI: 10.1101/2020.06.02.20106310. Shekhar R, Sheikh AB, Upadhyay S, et al. (2020) Early experience with COVID-19 patients at academic hospital in Southwestern United States. Infectious Diseases 52(8): 596-599. DOI: 10.1080/23744235.2020.1774645.

Shi H, Zuo Y, Yalavarthi S, et al. (2020) Neutrophil calprotectin identifies severe pulmonary disease in COVID-19. medRxiv: 2020.05.06.20093070. DOI: 10.1101/2020.05.06.20093070.

Shi P, Ren G, Yang J, et al. (2020) Clinical characteristics of imported and second-generation COVID-19 cases outside Wuhan, China: A multicenter retrospective study. medRxiv: 2020.04.19.20071472. DOI: 10.1101/2020.04.19.20071472. Shi Q, Zhao K, Yu J, et al. (2020) Clinical characteristics of 101 COVID-19 nonsurvivors in Wuhan, China: A retrospective study. medRxiv: 2020.03.04.20031039. DOI: 10.1101/2020.03.04.20031039.

Shi Y, Yu X, Zhao H, et al. (2020) Host susceptibility to severe COVID-19 and establishment of a host risk score: Findings of 487 cases outside Wuhan. Critical Care 24(1): 108. DOI: 10.1186/s13054-020-2833-7.

Shi Z, Resurreccion WK, Wang C-H, et al. (2020) Association of Cancer with Risk and Mortality of COVID-19: Results from the UK Biobank. medRxiv: 2020.07.10.20151076. DOI: 10.1101/2020.07.10.20151076. 
Sierpiński R, Pinkas J, Jankowski M, et al. (2020) Sex differences in the frequency of gastrointestinal symptoms and olfactory or taste disorders among 1,942 non-hospitalized patients with COVID-19. Polish Archives of Internal Medicine. DOI: 10.20452/pamw.15414.

Sigel K, Swartz T, Golden E, et al. (n.d.) Covid-19 and People with HIV Infection: Outcomes for Hospitalized Patients in New York City. Clinical Infectious Diseases. DOI: 10.1093/cid/ciaa880.

Silva Neto PV da, Carvalho JCS de, Pimentel VE, et al. (2020) Prognostic value of sTREM-1 in COVID-19 patients: A biomarker for disease severity and mortality. preprint, September. Infectious Diseases (except HIV/AIDS). DOI:

10.1101/2020.09.22.20199703.

Simons D, Brown J, Shahab L, et al. (2020) Smoking and COVID-19: Rapid evidence review for the Royal College of Physicians, London (UK). Qeios. DOI: 10.32388/VGJCUN.

Simons D, Perski O, Shahab L, et al. (2020) The association of smoking status with hospitalisation for COVID-19 compared with other respiratory viruses a year previous: A case-control study at a single UK National Health Service trust. medRxiv: 2020.11.26.20238469. DOI: 10.1101/2020.11.26.20238469.

Simons D, Shahab L, Brown J, et al. (2020) The association of smoking status with SARS-CoV-2 infection, hospitalisation and mortality from COVID-19: A living rapid evidence review with Bayesian meta-analyses (version 7). Addiction $\mathrm{n} / \mathrm{a}(\mathrm{n} / \mathrm{a})$. DOI: 10.1111/add.15276.

Singh BM, Bateman J, Viswanath A, et al. (2020) The risk of COVID hospital admission and COVID mortality during the first COVID 19 wave with a special emphasis on Ethnic Minorities: An observational study of a single, deprived, multi ethnic UK health economy. preprint, November. Infectious Diseases (except HIV/AIDS). DOI:

\subsection{1/2020.11.20.20224691.}

Sisó-Almirall A, Kostov B, Mas-Heredia M, et al. (2020) PROGNOSTIC FACTORS IN SPANISH COVID-19 PATIENTS: A CASE SERIES FROM BARCELONA. medRxiv: 2020.06.18.20134510. DOI: $\underline{10.1101 / 2020.06 .18 .20134510}$.

Soares R de CM, Mattos LR and Raposo LM (2020) Risk Factors for Hospitalization and Mortality due to COVID-19 in Espírito Santo State, Brazil.: tpmd200483. DOI: 10.4269/ajtmh.20-0483.

Solís P and Carreño H (2020) COVID-19 Fatality and Comorbidity Risk Factors among Diagnosed Patients in Mexico. DOI: $10.1101 / 2020.04 .21 .20074591$.

Soto-Mota A, Garza BAM, Rodriguez EM, et al. (2020) THE LOW-HARM SCORE FOR PREDICTING MORTALITY IN PATIENTS DIAGNOSED WITH COVID-19: A MULTICENTRIC VALIDATION STUDY. medRxiv: 2020.05.26.20111120. DOI: $10.1101 / 2020.05 .26 .20111120$.

Sourij H, Aziz F, Bräuer A, et al. (2020) COVID-19 fatality prediction in people with diabetes and prediabetes using a simple score upon hospital admission. Diabetes, Obesity and Metabolism: dom.14256. DOI: 10.1111/dom.14256. Souza FSH, Hojo-Souza NS, Santos EB, et al. (2020) Predicting the disease outcome in COVID-19 positive patients through Machine Learning: A retrospective cohort study with Brazilian data. medRxiv: 2020.06.26.20140764. DOI: 10.1101/2020.06.26.20140764.

Swiss Federal Statiscs Office (FSO) (2020) La part des gros fumeurs s'est réduite de moitié en 25 ans - Enquête suisse sur la santé 2017: Consommation de tabac Communiqué de presse. Office fédéral de la statistique. Available at: /content/bfs/fr/home/aktuell/neue-veroeffentlichungen.assetdetail.11907023.html (accessed 23 February 2021). 
Stead LF, Buitrago D, Preciado N, et al. (2013) Physician advice for smoking cessation. Cochrane Database of Systematic Reviews 2017(12). DOI: 10.1002/14651858.CD000165.pub4.

Sterlin D, Mathian A, Miyara M, et al. (2020) IgA dominates the early neutralizing antibody response to SARS-CoV-2. medRxiv: 2020.06.10.20126532. DOI: 10.1101/2020.06.10.20126532.

Strangfeld A, Schäfer M, Gianfrancesco MA, et al. (2021) Factors associated with COVID-19-related death in people with rheumatic diseases: Results from the COVID-19 Global Rheumatology Alliance physician-reported registry. Annals of the Rheumatic Diseases: annrheumdis-2020-219498. DOI: 10.1136/annrheumdis-2020-219498.

Suleyman G, Fadel RA, Malette KM, et al. (2020) Clinical Characteristics and Morbidity Associated With Coronavirus Disease 2019 in a Series of Patients in Metropolitan Detroit. JAMA Network Open 3(6): e2012270-e2012270. DOI: 10.1001/jamanetworkopen.2020.12270.

Sun L, Surya S, Le AN, et al. (2021) Rates of COVID-19-Related Outcomes in Cancer Compared With Noncancer Patients. JNCI Cancer Spectrum 5(1): pkaa120. DOI: 10.1093/jncics/pkaa120.

Talavera B, García-Azorín D, Martínez-Pías E, et al. (2020) Anosmia is associated with lower in-hospital mortality in COVID-19. Journal of the Neurological Sciences 419: 117163. DOI: 10.1016/i.jns.2020.117163.

Tao P-Y, Leng L, Liu K, et al. (2020) Determination of risk factors for predicting the onset of symptoms in asymptomatic COVID-19 infected patients. Int J Med Sci 17: 2187-2193. DOI: 10.7150/ijms.47576.

Tardif J-C, Bouabdallaoui N, L'Allier PL, et al. (2021) Efficacy of Colchicine in Non-Hospitalized Patients with COVID-19. DOI: $10.1101 / 2021.01 .26 .21250494$.

Targher G, Mantovani A, Wang XB, et al. (2020) Patients with diabetes are at higher risk for severe illness from COVID-19. Diabetes \& Metabolism. DOI: 10.1016/j.diabet.2020.05.001.

Tavakol Z, Ghannadi S, Tabesh MR, et al. (2021) Relationship between physical activity, healthy lifestyle and COVID-19 disease severity; a cross-sectional study. J. public health NA: 1-9. DOI: 10.1007/s10389-020-01468-9.

Team RC (2013) The R Project for Statistical Computing.: 1-12.

Thakur K, Sagayaraj A, Prasad KC, et al. (2021) Olfactory Dysfunction in COVID-19 Patients: Findings from a Tertiary Rural Centre. Indian j. otolaryngol. head neck surg NA: 1-7. DOI: 10.1007/s12070-021-02364-8.

Thiabaud A, Iten A, Balmelli C, et al. (2020) SARS-CoV-2/COVID-19 hospitalised patients in Switzerland: A prospective cohort profile. preprint, December. Epidemiology. DOI: 10.1101/2020.12.10.20246884.

Thompson JV, Meghani N, Powell BM, et al. (2020) Patient characteristics and predictors of mortality in 470 adults admitted to a district general hospital in England with Covid-19. medRxiv: 2020.07.21.20153650. DOI:

10.1101/2020.07.21.20153650.

Torres-Macho J, Ryan P, Valencia J, et al. (2020) The PANDEMYC Score. An Easily Applicable and Interpretable Model for Predicting Mortality Associated With COVID-19. Journal of Clinical Medicine 9(10): 3066. DOI: 10.3390/jcm9103066. Tricco AC, Antony J, Zarin W, et al. (2015) A scoping review of rapid review methods. BMC Medicine 13(1): 224. DOI: 10.1186/s12916-015-0465-6.

Trubiano JA, Vogrin S, Smibert OC, et al. (2020) COVID-MATCH65 - A prospectively derived clinical decision rule for severe acute respiratory syndrome coronavirus 2. medRxiv: 2020.06.30.20143818. DOI: $10.1101 / 2020.06 .30 .20143818$. Ugur Chousein EG, Cortuk M, Cinarka H, et al. (2020) Is there any effect of smoking status on severity and mortality of 
hospitalized patients with COVID-19 pneumonia? Tuberk. Toraks 68: 371-378. DOI: 10.5578/tt.70352.

Ullah AZMD, Sivapalan L, Chelala C, et al. (2020) COVID-19 in patients with hepatobiliary and pancreatic diseases in East London: A single-centre cohort study. medRxiv: 2020.09.07.20189621. DOI: 10.1101/2020.09.07.20189621.

Vahidy FS, Pan AP, Ahnstedt H, et al. (2021) Sex differences in susceptibility, severity, and outcomes of coronavirus disease 2019: Cross-sectional analysis from a diverse US metropolitan area. PloS one 16. DOI:

10.1371/journal.pone.0245556.

Valenti L, Bergna A, Pelusi S, et al. (2020) SARS-CoV-2 seroprevalence trends in healthy blood donors during the COVID-19 Milan outbreak. medRxiv: 2020.05.11.20098442. DOI: 10.1101/2020.05.11.20098442.

Valenzuela O, Ibanez SE, Poli M, et al. (2020) First report of tocilizumab use in a cohort of Latin American patients hospitalized for severe COVID-19 pneumonia. medRxiv: 2020.08.12.20173104. DOI: 10.1101/2020.08.12.20173104. Valle DMD, Kim-schulze S, Hsin-hui H, et al. (2020) An inflammatory cytokine signature helps predict COVID-19 severity and death. medRxiv: 2020.05.28.20115758. DOI: $10.1101 / 2020.05 .28 .20115758$.

Vaquero LM, Barrado MES, Escobar D, et al. (2020) C-Reactive protein and SOFA score as early predictors of critical care requirement in patients with COVID-19 pneumonia in Spain. medRxiv: 2020.05.22.20110429. DOI:

10.1101/2020.05.22.20110429.

Vardavas Cl and Nikitara K (2020) COVID-19 and smoking: A systematic review of the evidence. Tob Induc Dis 18: 20. DOI: $10.18332 /$ tid/119324.

Veras FP, Pontelli M, Silva C, et al. (2020) SARS-CoV-2 triggered neutrophil extracellular traps (NETs) mediate COVID-19 pathology. medRxiv: 2020.06.08.20125823. DOI: 10.1101/2020.06.08.20125823.

Vila-Corcoles A, Satue-Gracia E, Vila-Rovira A, et al. (2020) COVID-19 TARRACO Cohort Study: Development of a predictive prognostic rule for early assessment of COVID-19 patients in primary care settings. preprint, December. Infectious Diseases (except HIV/AIDS). DOI: 10.1101/2020.12.11.20247932.

Vila-Corcoles A, Satue-Gracia E, Vila-Rovira A, et al. (2021) COVID19-related and all-cause mortality among middle-aged and older adults across the first epidemic wave of SARS-COV-2 infection in the region of Tarragona, Spain: Results from the COVID19 TARRACO Cohort Study, March-June 2020. DOI: 10.1101/2021.02.02.21251028.

Vila-Córcoles A, Ochoa-Gondar O, Satué-Gracia EM, et al. (2020) Influence of prior comorbidities and chronic medications use on the risk of COVID-19 in adults: A population-based cohort study in Tarragona, Spain. BMJ Open 10(12): e041577. DOI: 10.1136/bmjopen-2020-041577.

Villar-Garcia J, Vivanco-Hidalgo RM, Cleries M, et al. (2020) Risk factors for SARS-CoV-2 infection, hospitalisation, and death in Catalonia, Spain: A population-based cross-sectional study. medRxiv: 2020.08.26.20182303. DOI:

10.1101/2020.08.26.20182303.

Wan S, Xiang Y, Fang W, et al. (2020) Clinical features and treatment of COVID-19 patients in northeast Chongqing. Journal of Medical Virology 92(7): 797-806. DOI: 10.1002/jmv.25783.

Wang A-L, Zhong X and Hurd Y (2020) Comorbidity and Sociodemographic determinants in COVID-19 Mortality in an US Urban Healthcare System. medRxiv: 2020.06.11.20128926. DOI: 10.1101/2020.06.11.20128926.

Wang B, Oekelen OV, Mouhieddine T, et al. (2020) A tertiary center experience of multiple myeloma patients with COVID19: Lessons learned and the path forward. medRxiv: 2020.06.04.20122846. DOI: $10.1101 / 2020.06 .04 .20122846$. 
Wang JG, Liu B, Percha B, et al. (2020) Cardiovascular disease and severe hypoxemia associated with higher rates of non-invasive respiratory support failure in COVID-19. DOI: 10.1101/2020.09.27.20202747.

Wang R, Pan M, Zhang X, et al. (2020) Epidemiological and clinical features of 125 Hospitalized Patients with COVID-19 in Fuyang, Anhui, China. International Journal of Infectious Diseases 95: 421-428. DOI: 10.1016/j.ijid.2020.03.070. Wang Z, Zheutlin A, Kao Y-H, et al. (2020) Hospitalised COVID-19 patients of the Mount Sinai Health System: A retrospective observational study using the electronic medical records. BMJ Open 10(10): e040441. DOI:

\subsection{6/bmjopen-2020-040441.}

Wang Z-H, Shu C, Ran X, et al. (2020) Critically III Patients with Coronavirus Disease 2019 in a Designated ICU: Clinical Features and Predictors for Mortality. Risk Management and Healthcare Policy Volume 13: 833-845. DOI:

\subsection{7/RMHP.S263095.}

Ward H, Atchison CJ, Whitaker M, et al. (2020) Antibody prevalence for SARS-CoV-2 in England following first peak of the pandemic: REACT2 study in 100,000 adults. medRxiv: 2020.08.12.20173690. DOI: 10.1101/2020.08.12.20173690. Weerahandi H, Hochman KA, Simon E, et al. (2020) Post-discharge health status and symptoms in patients with severe COVID-19. medRxiv: 2020.08.11.20172742. DOI: 10.1101/2020.08.11.20172742.

Wei W, Ortwine JK, Mang NS, et al. (2020) Limited Role for Antibiotics in COVID-19: Scarce Evidence of Bacterial Coinfection. medRxiv: 2020.06.16.20133181. DOI: 10.1101/2020.06.16.20133181.

Williamson EJ, Walker AJ, Bhaskaran K, et al. (2020) Factors associated with COVID-19-related death using OpenSAFELY. Nature 584(7821): 430-436. DOI: 10.1038/s41586-020-2521-4.

Woolcott OO and Castilla-Bancayán JP (2020) Diabetes and Mortality Among 1.6 Million Adult Patients Screened for SARS-CoV-2 in Mexico. preprint, November. Infectious Diseases (except HIV/AIDS). DOI: 10.1101/2020.11.25.20238345. Woolford SJ, D'Angelo S, Curtis EM, et al. (2020) COVID-19 and associations with frailty and multimorbidity: A prospective analysis of UK Biobank participants. Aging Clinical and Experimental Research 32(9): 1897-1905. DOI: 10.1007/s40520020-01653-6.

Wu MA, Fossali T, Pandolfi L, et al. (2020) COVID-19: The key role of pulmonary capillary leakage. An observational cohort study. medRxiv: 2020.05.17.20104877. DOI: 10.1101/2020.05.17.20104877.

Xie Y, Chen S, Wang X, et al. (2020) Early Diagnosis and Clinical Significance of Acute Cardiac Injury - Under the Iceberg: A Retrospective Cohort Study of 619 Non-critically III Hospitalized COVID-19 Pneumonia Patients. medRxiv: 2020.07.06.20147256. DOI: 10.1101/2020.07.06.20147256.

Xu H, Hou K, Xu H, et al. (2020) Acute Myocardial Injury of Patients with Coronavirus Disease 2019. medRxiv: 2020.03.05.20031591. DOI: 10.1101/2020.03.05.20031591.

Yadaw AS, Li Y-c, Bose S, et al. (2020) Clinical features of COVID-19 mortality: Development and validation of a clinical prediction model. The Lancet Digital Health 2(10): e516-e525. DOI: 10.1016/S2589-7500(20)30217-X.

Yan H, Valdes AM, Vijay A, et al. (2020) Role of Drugs used for chronic disease management on Susceptibility and Severity of COVID-19: A Large Case-Control Study. medRxiv: 2020.04.24.20077875. DOI: 10.1101/2020.04.24.20077875. Yang X, Yu Y, Xu J, et al. (2020) Clinical course and outcomes of critically ill patients with SARS-CoV-2 pneumonia in Wuhan, China: A single-centered, retrospective, observational study. The Lancet Respiratory Medicine 8(5): 475-481. DOI: $10.1016 /$ S2213-2600(20)30079-5. 
Yanover C, Mizrahi B, Kalkstein N, et al. (2020) What factors increase the risk of complications in SARS-CoV-2 positive patients? A cohort study in a nationwide Israeli health organization. medRxiv: 2020.05.07.20091652. DOI:

\section{$10.1101 / 2020.05 .07 .20091652$.}

Yao K, Hasegawa S, Tagashira Y, et al. (2021) Experience of 101 patients with coronavirus infectious disease 2019 (COVID-19) at a tertiary care center in Japan. Journal of Infection and Chemotherapy 27(2): 413-417. DOI:

\subsection{6/i.jiac.2020.11.024.}

Yao Q, Wang $P$, Wang $X$, et al. (n.d.) A retrospective study of risk factors for severe acute respiratory syndrome coronavirus 2 infections in hospitalized adult patients. DOI: 10.20452/pamw.15312.

Yin R, Yang Z, Wei Y, et al. (2020) Clinical characteristics of 106 patients with neurological diseases and co-morbid coronavirus disease 2019: A retrospective study. medRxiv: 2020.04.29.20085415. DOI: $10.1101 / 2020.04 .29 .20085415$. Yoo E, Percha B, Tomlinson M, et al. (2020) Development and calibration of a simple mortality risk score for hospitalized COVID-19 adults. medRxiv: 2020.08.31.20185363. DOI: 10.1101/2020.08.31.20185363.

Yu T, Cai S, Zheng Z, et al. (2020) Association Between Clinical Manifestations and Prognosis in Patients with COVID-19. Clinical Therapeutics 42(6): 964-972. DOI: 10.1016/j.clinthera.2020.04.009.

Zacharioudakis IM, Prasad PJ, Zervou FN, et al. (2020) Association of SARS-CoV-2 Genomic Load with COVID-19 Patient Outcomes. medRxiv: 2020.07.02.20145151. DOI: 10.1101/2020.07.02.20145151.

Zeng H, Zhang T, He X, et al. (2020) Impact of Chronic Comorbidities on Progression and Prognosis in Patients with COVID-19: A Retrospective Cohort Study in 1031 Hospitalized Cases in Wuhan, China. medRxiv: 2020.06.14.20125997. DOI: $10.1101 / 2020.06 .14 .20125997$.

Zhan T, Liu M, Tang Y, et al. (2020) Retrospective analysis of clinical characteristics of 405 patients with COVID-19. Journal of International Medical Research 48(8): 030006052094903. DOI: 10.1177/0300060520949039.

Zhan Z, Yang X, Du H, et al. (2020) Early Improvement of Acute Respiratory Distress Syndrome in Patients with COVID19: Insights from the Data of ICU Patients in Chongqing, China. medRxiv: 2020.07.15.20154047. DOI:

\subsection{1/2020.07.15.20154047.}

Zhang J, Cao Y, Tan G, et al. (2020) Clinical, radiological, and laboratory characteristics and risk factors for severity and mortality of 289 hospitalized COVID-19 patients. Allergy: all.14496. DOI: 10.1111/all.14496.

Zhang J-j, Dong X, Cao Y-y, et al. (2020) Clinical characteristics of 140 patients infected with SARS-CoV-2 in Wuhan, China. Allergy 75(7): 1730-1741. DOI: 10.1111/all.14238.

Zhang Q, Wang Z, Lv Y, et al. (2021) Clinical features and prognostic factors of patients with COVID-19 in Henan Province, China. Hum Cell NA. DOI: 10.1007/s13577-021-00499-y.

Zhang X, Cai H, Hu J, et al. (2020) Epidemiological, clinical characteristics of cases of SARS-CoV-2 infection with abnormal imaging findings. International Journal of Infectious Diseases 94: 81-87. DOI: 10.1016/j.jijid.2020.03.040. Zhang X, Li X, Sun Z, et al. (2020) Physical activity and COVID-19: An observational and Mendelian randomisation study. Journal of Global Health 10(2): 020514. DOI: 10.7189/jogh-10-020514.

Zhang Y, Yang H, Li S, et al. (2021) Association analysis framework of genetic and exposure risks for COVID-19 in middleaged and elderly adults. Mechanisms of Ageing and Development 194: 111433. DOI: 10.1016/j.mad.2021.111433.

Zhao Z, Chen A, Hou W, et al. (2020) Prediction model and risk scores of ICU admission and mortality in COVID-19. PLOS 
ONE 15(7): e0236618. DOI: 10.1371/journal.pone.0236618.

Zheng KI, Gao F, Wang X-B, et al. (2020) Letter to the Editor: Obesity as a risk factor for greater severity of COVID-19 in patients with metabolic associated fatty liver disease. Metabolism 108: 154244. DOI: 10.1016/.j.metabol.2020.154244. Zheng Y, Xiong C, Liu Y, et al. (2020) Epidemiological and clinical characteristics analysis of COVID-19 in the surrounding areas of Wuhan, Hubei Province in 2020. Pharmacological Research 157: 104821. DOI: 10.1016/.jphrs.2020.104821. Zhong R, Chen L, Zhang Q, et al. (2021) Which Factors, Smoking, Drinking Alcohol, Betel Quid Chewing, or Underlying Diseases, Are More Likely to Influence the Severity of COVID-19? Frontiers in Physiology 11: 623498. DOI: 10.3389/fphys.2020.623498.

Zhou F, Yu T, Du R, et al. (2020) Clinical course and risk factors for mortality of adult inpatients with COVID-19 in Wuhan, China: A retrospective cohort study. The Lancet 395(10229): 1054-1062. DOI: 10.1016/S0140-6736(20)30566-3. Zhou J, Ma Y, Liu Y, et al. (2021) A Correlation Analysis between the Nutritional Status and Prognosis of COVID-19 Patients. J Nutr Health Aging 25: 84-93. DOI: 10.1007/s12603-020-1457-6.

Zhou K, Sun Y, Li L, et al. (2020) Eleven Routine Clinical Features Predict COVID-19 Severity. medRxiv: 2020.07.28.20163022. DOI: 10.1101/2020.07.28.20163022.

Zhou W, Song L, Wang X, et al. (2020) Cardiac injury prediction and lymphocyte immunity and inflammation analysis in hospitalized patients with coronavirus disease 2019 (COVID-19). International Journal of Cardiology: S016752732034002X. DOI: 10.1016/j.ijcard.2020.10.049.

Zhou W, Qin X, Hu X, et al. (2020) Prognosis models for severe and critical COVID-19 based on the Charlson and Elixhauser comorbidity indices. International Journal of Medical Sciences 17(15): 2257-2263. DOI: 10.7150/ijms.50007. Zhou Y, He Y, Yang H, et al. (2020) Exploiting an early warning Nomogram for predicting the risk of ICU admission in patients with COVID-19: A multi-center study in China. Scandinavian Journal of Trauma, Resuscitation and Emergency Medicine 28(1): 106. DOI: 10.1186/s13049-020-00795-w.

Zhou Y, He X, Zhang J, et al. (2020) Prolonged SARS-CoV-2 Viral Shedding in Patients with COVID-19 was Associated with Delayed Initiation of Arbidol Treatment: A retrospective cohort study. medRxiv: 2020.06.09.20076646. DOI:

\subsection{1/2020.06.09.20076646.}

Zhu S, Gao Q, Yang L, et al. (2020) Prevalence and risk factors of disability and anxiety in a retrospective cohort of 432 survivors of Coronavirus Disease-2019 (Covid-19) from China. PLOS ONE Federici S (ed.) 15(12): e0243883. DOI: 10.1371/journal.pone.0243883.

Ziehr DR, Alladina J, Petri CR, et al. (2020) Respiratory Pathophysiology of Mechanically Ventilated Patients with COVID19: A Cohort Study. American Journal of Respiratory and Critical Care Medicine 201(12): 1560-1564. DOI:

10.1164/rccm.202004-1163LE.

Zinellu A, Arru F, De Vito A, et al. (2021) The De Ritis ratio as prognostic biomarker of in-hospital mortality in COVID-19 patients. European Journal of Clinical Investigation 51(1). DOI: 10.1111/eci.13427.

Zobairy H, Shamsoddin E, Rasouli MA, et al. (2020) Association of olfactory dysfunction with hospitalization for COVID-19: A multicenter study in Kurdistan. medRxiv: 2020.07.26.20158550. DOI: 10.1101/2020.07.26.20158550.

Zuo Y, Yalavarthi S, Shi H, et al. (2020) Neutrophil extracellular traps in COVID-19. JCI Insight. DOI: 10.1172/jici.insight.138999. 
Zuo Y, Warnock M, Harbaugh A, et al. (2020) Plasma tissue plasminogen activator and plasminogen activator inhibitor-1 in hospitalized COVID-19 patients. preprint, September. Infectious Diseases (except HIV/AIDS). DOI: 10.1101/2020.08.29.20184358.

Zuo Y, Estes SK, Gandhi AA, et al. (2020) Prothrombotic antiphospholipid antibodies in COVID-19. medRxiv: 2020.06.15.20131607. DOI: 10.1101/2020.06.15.20131607. 